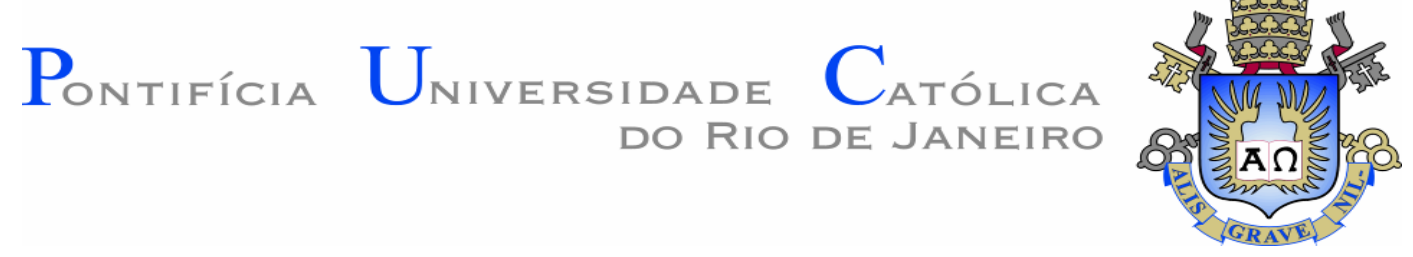

Thiago Ribeiro da Motta

Uma Avaliação de Filtros 2D para Remoção de Ruído Speckle em Exames de Ultrassom

Dissertação de Mestrado

Dissertação apresentada ao Programa de Pósgraduação em Informática da PUC-Rio como requisito parcial para obtenção do grau de Mestre em Informática.

Orientador: Prof. Alberto Barbosa Raposo

Rio de Janeiro Abril de 2017 
Pontifícia Univergidade $\begin{gathered}\text { Catálica } \\ \text { Do Rio De Janiro }\end{gathered}$

Thiago Ribeiro da Motta

\title{
Uma Avaliação de Filtros 2D para Remoção de Ruído Speckle em Exames de Ultrassom
}

Dissertação apresentada como requisito parcial para obtenção do grau de Mestre pelo Programa de PósGraduação em Informática da PUC-Rio. Aprovada pela Comissão Examinadora abaixo assinada.

\author{
Prof. Alberto Barbosa Raposo \\ Orientador \\ Departamento de Informática - PUC-Rio
}

Prof. Hélio Côrtes Vieira Lopes

Departamento de Informática - PUC-Rio

DSc. Manuel Eduardo Loaiza Fernández

PUC-Rio

Prof. Marcio da Silveira Carvalho

Coordenador(a) Setorial do Centro Técnico Científico - PUC-Rio 
Todos os direitos reservados. É proibida a reprodução total ou parcial do trabalho sem autorização da universidade, do autor e do orientador.

\section{Thiago Ribeiro da Motta}

Graduou-se no curso de Engenharia de Computação pela PUC-Rio em 2014. Já trabalhou nas áreas de Realidade Virtual e Aumentada, Projeção Mapeada e Visualização nas áreas de Petróleo e Gás.

Ficha Catalográfica

Motta, Thiago Ribeiro da

Uma avaliação de filtros 2D para remoção de ruído speckle em exames de ultrassom / Thiago Ribeiro da Motta; orientador: Alberto Barbosa Raposo. - 2017.

127 f. : il. color. ; $30 \mathrm{~cm}$

Dissertação (mestrado)-Pontifícia Universidade Católica do Rio de Janeiro, Departamento de Informática, 2017.

Inclui bibliografia

1. Informática - Teses. 2. Ruído speckle. 3. Exame de ultrassom. 4. Preservação de arestas. 5. Avaliação de filtros 2D. 6. Escolha de parâmetros. I. Raposo, Alberto Barbosa. II. Pontifícia Universidade Católica do Rio de Janeiro. Departamento de Informática. III. Título. 
Dedico este trabalho ao Peter Hohl, por ter acreditado em mim desde o primeiro dia em que cheguei na área acadêmica, por ter me feito sentir em casa quando cheguei ao Tecgraf e por sempre garantir um sorriso na vida de todos que passaram por ele. Onde quer que você esteja, que seja repleto de luz e paz! 


\section{Agradecimentos}

Agradeço ao Professor Alberto pela confiança e liberdade apresentados durante a confecção deste trabalho.

Ao Manuel Loaiza pela disposição de ouvir novas ideias e a discuti-las, pelas discussões científicas e ensinamentos.

Aos professores do Departamento de Informática pelos ensinamentos, orientações e ajuda.

Em especial à professora Simone Diniz pelo direcionamento ao Tecgraf e à área acadêmica.

Agradeço ao Daniel Radetic por todo o tempo despendido para me ajudar com diversos temas abordados nesta pesquisa.

E finalmente aos bons amigos por sempre me inspirarem a ser uma pessoa melhor. 


\section{Resumo}

Motta, Thiago Ribeiro da; Raposo, Alberto Barbosa. Uma Avaliação de Filtros 2D para Remoção de Ruído Speckle em Exames de Ultrassom. Rio de Janeiro, 2017, 127p. Dissertação de Mestrado - Departamento de Informática, Pontifícia Universidade Católica do Rio de Janeiro.

Exames de ultrassom são uma ferramenta popular de aquisição de imagens na medicina atual por ser um procedimento não-invasivo, seguro e barato. Entretanto, inerente a qualquer exame de ultrassom encontra-se o ruído speckle, responsável pela degradação da imagem e dificultando tanto sua interpretação por parte de médicos e pacientes, quanto prejudicando a acurácia de métodos computacionais de pós processamento, como classificação, reconstrução, caracterização de tecidos e segmentação, entre outros. Portanto, métodos de remoção ou suavização deste ruído que preservem as principais características do conteúdo observado se fazem fundamentais para um avanço nestes processos. Definido como um ruído multiplicativo, que segue estatísticas não-Gaussianas e como fortemente correlacionado, sua solução ainda hoje é tema de debates e estudos. Neste trabalho apresentaremos diversos métodos de filtragem 2D que se propõem a reduzir ou solucionar o ruído speckle bem como métodos qualitativos para avaliar seus desempenhos e técnicas para escolher os melhores parâmetros de cada filtro a fim de eleger quais métodos melhor solucionam este ruído.

\section{Palavras-chave}

Ruído speckle; exame de ultrassom; preservação de arestas; avaliação de filtros 2D; escolha de parâmetros; métricas qualitativas de imagem; $k$-means. 
Abstract

Motta, Thiago Ribeiro da; Raposo, Alberto Barbosa (advisor). An Evaluation of 2D Filters for Speckle Denoising Ultrasound Exams. Rio de Janeiro, 2017, 127p. Dissertação de Mestrado - Departamento de Informática, Pontifícia Universidade Católica do Rio de Janeiro.

Ultrasound exams are a popular tool for image acquisition in day-to-day medicine, since it is a noninvasive, safe and cheap procedure. However, speckle noise is intrinsic to any ultrasound exam, and it is responsible for image quality degradation and for hindering its interpretation by doctors and patients alike, while also impairing the accuracy of post processing computational methods, such as classification, reconstruction, tissue characterization and segmentation, among others. Hence, smoothing or denoising methods that preserves the observed content core attributes are essential for those processes. Defined as a multiplicative noise, following non-Gaussian statistics and as strongly correlated, its solution today is still a matter of debates and research. In this work, several 2D filters that aim to smooth or remove speckle noise along with qualitative methods to evaluate their performances and means of choosing their best parameters are presented.

\section{Keywords}

Speckle noise; ultrasound exams; edge preservation; 2D filters evaluation; parameters estimation; qualitative image metrics, $k$-means. 


\section{Sumário}

1. Introdução 18

$\begin{array}{lr}\text { 1.1. Motivação } & 19\end{array}$

1.2. Objetivo 19

$\begin{array}{ll}\text { 1.3. Estrutura } & 19\end{array}$

2. Fundamentação Teórica 21

2.1. Imagens por Ultrassom 21

2.2. Ruído Speckle 23

2.3. Filtros 24

2.4. Métricas 25

2.5. Avaliações 25

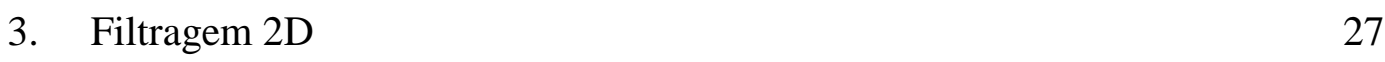

3.1. Filtros lineares 30

3.1.1. Filtros estatísticos de primeira ordem 30

3.1.1.1. Mean and variance local statistics Filter (DsFlsmv) 30

3.1.1.2. Wiener Despeckle Filter (DsFwiener2) 30

3.1.2. Minimum speckle index homogeneous Mask Area Filtering (DsFlsminsc)31

3.2. Filtros não lineares 31

3.2.1. Hybrid Median Filtering (DsFmedian) 31

3.2.2. Linear Scaling of the Gray-Levels Filter (DsFca) 31

3.2.3. Linear Scaling Filter (DsFls) 32

3.2.4. Linear Scaling and Sorting Filter (DsFlecasort) 32

3.2.5. Maximum Homogeneity Over Pixel Neighborhood Filtering (DsFhomog) 32

3.2.6. Geometric Filtering (DsFgf4d) 32

3.2.7. Homomorphic Filtering (DsFhomo) 33

3.2.8. Denoising Sign Dependent Noise (DSDN) 33

3.2.9. Shape-Adaptive Discrete Cosine Transform (SADCTD) 33

3.3. Filtros de suavização de ruído Speckle por Difusão 34

3.3.1. Anisotropic Diffusion Filtering (DsFad) 34

3.3.2. Speckle-Reducing Anisotropic Diffusion Filtering (DsFsrad) 34

3.3.3. Nonlinear Coherent Anisotropic Diffusion Filtering (DsFlndif) 35

3.3.4. Bilateral $\quad 35$

3.3.5. Trilateral 36

3.4. Outros 37

3.4.1. Field of Experts with a Nakagami Distribution (FoE_Naka) 37

3.4.2. Wavelet Filtering (DsFwaveltc) 38

3.4.3. L0 Smoothing 38

3.4.4. Bilateral and L0 Smoothing 39

3.4.5. Probabilistic Patch Based (PPB_Nakagami) 39

3.4.6. Synthetic Aperture Radar Block Matching 3D (SARBM3D) 40

4. Métricas 43

4.1. Tipos de Métricas $\quad 43$

4.1.1. Referência Completa 43

4.1.1.1. Average Difference (AD) 44

4.1.1.2. Mean Square Error (MSE) 44

4.1.1.3. Momentos de Minkowski M3 e M4 44 
4.1.1.4. Signal-to-Noise Error (SNR) 45

4.1.1.5. Universal Quality Index (UQI) 45

4.1.1.6. Structural Similarity Index Map (SSIM)

4.1.1.7. Structural Content (SC) 45

4.1.1.8. Normalized Cross-Correlation (NK) 46

4.1.1.9. Maximum Difference (MD) 46

4.1.1.10. Laplacian Mean Squared Error (LMSE) 46

4.1.1.11. Normalized Absolute Error (NAE) 46

4.1.1.12. Coefficient of Correlation(COC) 47

4.1.1.13. Pratt Figure of Merit (FOM) 47

4.1.2. Referência Reduzida $\quad 47$

4.1.2.1. Beta 47

4.1.2.2. Speckle Supression Index (SSI) 48

4.1.2.3. Speckle Suppression and Mean Preservation Index (SMPI) 48

4.1.2.4. Edge Save Index (ESI) 48

4.1.2.5. Comparison Quality (CQ) 49

4.1.2.6. Comparison Texture Quality (CTQ) 50

4.1.3. Sem Referência $\quad 50$

4.1.3.1. MetricQ (Q) 50

4.2. Categorias de Limites das Métricas 51

4.2.1. Min-Max 52

4.2.2. Centered 53

4.2.3. Zero 53

4.3. Tipos de Pontuação 54

5. Escolha dos parâmetros dos Filtros 2D 56

$\begin{array}{ll}\text { 5.1. Análises por gráfico } & 57\end{array}$

$\begin{array}{ll}\text { 5.2. k-Means } & 59\end{array}$

6. Avaliação 64

6.1. Setup de testes 64

6.2. Escolha do total de Clusters $\quad 65$

6.3. Cardinalidade Relativa x Performance 65

6.4. Clusterização x Análise por Gráfico 66

6.5. Tipos de Pontuação 66

6.6. Clusterização sem Métricas de Agrupamento 67

$\begin{array}{ll}\text { 6.7. Análise normalizada das métricas } & 73\end{array}$

$\begin{array}{ll}\text { 6.8. Discussão } & 73\end{array}$

6.9. Parâmetros mais apropriados $\quad 74$

$\begin{array}{ll}\text { 6.10. Filtros } & 75 \\ 6.11 . \text { Metricas } & 78\end{array}$

$\begin{array}{ll}\text { 6.11. Métricas } & 78\end{array}$

7. Conclusão e trabalhos futuros 85

$\begin{array}{ll}\text { Referências bibliográficas } & 87\end{array}$

8. Anexo A 92

$\begin{array}{lr}\text { 9. Anexo B } & 110\end{array}$ 


\section{Lista de figuras}

Figura 2.1: O modelo usual de tecido em imagens de ultrassom[2].

Figura 2.2: (a) O espalhamento do raio de ultrassom. (b) Um pulso no tempo e no domínio da frequência [2].

Figura 2.3: Da esquerda para a direita: Ultrassom nos modos A, B e M (embaixo) [5].

Figura 3.1: Exemplo de filtros que atendem às características desejáveis mas violam os requisitos fundamentais. À esquerda estão as imagens originais e à direita suas versões filtradas, respectivamente. Em ambas as imagens filtradas é possível observar como regiões mais homogêneas foram criadas, entretanto, as estruturas circuladas em vermelho foram deformadas a ponto de perderem o significado que tinham antes. No caso as fronteiras foram drasticamente borradas a ponto de os exames aparentarem ter uma deformação física inexistente no exame original.

Figura 3.2 - Exemplo de filtros que atendem a ambos os requisitos fundamentais e características desejáveis. À esquerda estão as imagens não processadas e à direita suas versões filtradas, respectivamente.

Figura 3.3: Aproximações anisotrópicas locais, resultado da aplicação do algoritmo. Da esquerda para a direita: o estimador anisotrópico adaptativo; exemplos da aplicação do estimador. [31]

Figura 3.4 - Esquema do filtro PPB Iterativo [37].

Figura 3.5: Exemplo do passo de agrupamento em uma imagem artificial, em que cada bloco de referência possui outros blocos semelhantes [34].

Figura 5.1: Melhores resultados de cada imagem para cada variação de um único mesmo parâmetro. Os valores em torno do círculo representam a variação de todos os valores usados neste parâmetro, enquanto os valores na vertical representam a melhor pontuação obtida em uma métrica de agrupamento (FR para esta imagem). As linhas coloridas representam os 18 casos de teste abordados.

Figura 5.2: À esquerda: As melhores pontuações de todas as variações de um mesmo parâmetro, conforme visto na figura anterior. À direita: $\mathrm{O}$ eixo vertical representa o intervalo de pontuações atingido por esta configuração de parâmetros, enquanto o eixo horizontal contém a variação de um único parâmetro, no caso [3:2:19]. Os anéis significam as pontuações obtidas ao fixar o parâmetro visto no eixo vertical e variar os outros parâmetros deste filtro. Três gráficos são apresentados à direita para apresentar o comportamento médio obtido por este filtro, obtendo seus melhores resultados com os valores 3 e 5 neste parâmetro. As setas representam alguns dos elementos individuais tidos como outliers, presentes em cada teste.

Figura 5.3: Sobreposição dos gráficos individuais. Note como este gráfico possui uma maior concentração dos valores do parâmetro quando este é igual a 2 , com pontuações centralizadas no intervalo $(0.7,0.8)$, sendo este valor, portanto, uma boa indicação para este parâmetro.

Figura 5.4: Representação do método do "cotovelo" para determinar a melhor escolha do número total de clusters a serem usados no k-means [66]. Nesta imagem o valor de $\mathrm{k}=3$, circundado em vermelho, representa o total de clusters que melhor descreve os dados utilizados. 
Figura 5.5: Visualização do resultado da aplicação do k-means para todos os casos de teste e com somente o filtro DsFhomo, neste exemplo com apenas dois parâmetros (P1 e P2) e para o método de avaliação NR. A linha preta à direita representa o cluster com maior cardinalidade relativa, enquanto a linha preta à esquerda representa o cluster de maior performance. Cada combinação de cor e símbolo representam idealmente um cluster diferente, entretanto como existe um limite de 6 cores e 6 símbolos nesta representação e 40 clusters foram usados, quatro combinações estão repetidas.

Figura 5.6: Representação dos clusters de maior Cardinalidade Relativa e melhor Performance, respectivamente. Neste exemplo o DsFhomo continua sendo usado assim como somente a métrica NR é avaliada para simplificação. Perf representa o desempenho obtido pelo centroide deste cluster na métrica NR, enquanto Card representa a Cardinalidade Relativa atingida. P1 e P2 são os parâmetros utilizados por este filtro.

Figura 6.1: Diferentes avaliações sobre uma mesma imagem do filtro PPB_Nakagami. Card (imagens na primeira linha) representa a avaliação por Cardinalidade Relativa enquanto Perf (na segunda linha) representa a avaliação por Melhor Performance, CltAll (na terceira linha) a clusterização com todas as métricas de um mesmo grupo; Light e Weight são os tipos de pontuação com e sem peso, respectivamente; $\mathrm{kGap}$ representam as avaliações com o total de clusters calculados pelo Critério de Gap enquanto kSqrt representa método empírico; FR é respectivo à métrica de agrupamento Full Reference.

Figura 6.2: Avaliação da aplicação dos parâmetros encontrados pelo centroide de maior Cardinalidade Relativa ao filtro SADCTD. Os números do título de cada imagem representam os parâmetros usados no filtro, como apresentados no capítulo 3, com exceção das imagens Original e Ground Truth.

Figura 6.3: Avaliação da aplicação dos parâmetros encontrados pelo centroide de maior Performance ao filtro SADCTD. Os números do título de cada imagem representam os parâmetros usados no filtro, como apresentados no capítulo 3, com exceção das imagens Original e Ground Truth.

Figura 6.4: Análise por gráfico do filtro SADCTD de ambos os seus parâmetros sobre a pontuação FRD.

Figura 6.5: Análise de diferentes imagens de entrada feita pelo k-means de Cardinalidade Relativa segundo as 7 métricas de agrupamento testadas. Os números abaixo de cada imagem representam os parâmetros usados pelo filtro PPB_Nakagami para gerar este resultado. Um zoom de uma região de interesse pode ser visto no canto inferior direito de cada imagem para que a qualidade do filtro possa ser mais facilmente percebida.

Figura 8.1: Aplicação de todos os filtros apresentados com suas configurações de parâmetros definidas de acordo com as avaliações feitas ao longo do capítulo 6. No canto inferior direito de cada imagem existe uma área com zoom para que seja possível melhor visualizar o efeito de filtragem obtido.

Figura 10.1: Aplicações de todos os filtros discutidos no capítulo 3, com as configurações de parâmetros determinadas na seção 6.9 na imagem de teste Testcase 01-26. No canto inferior direito de cada imagem encontra-se uma região ampliada de uma área de interesse do exame que está sendo analisado.

Figura 10.2: Aplicações de todos os filtros discutidos no capítulo 3, com as configurações de parâmetros determinadas na seção 6.9 na imagem de teste 
Testcase 01-46. No canto inferior direito de cada imagem encontra-se uma região ampliada de uma área de interesse do exame que está sendo analisado.

Figura 10.3: Aplicações de todos os filtros discutidos no capítulo 3, com as configurações de parâmetros determinadas na seção 6.9 na imagem de teste Testcase 01-62. No canto inferior direito de cada imagem encontra-se uma região ampliada de uma área de interesse do exame que está sendo analisado.

Figura 10.4: Aplicações de todos os filtros discutidos no capítulo 3, com as configurações de parâmetros determinadas na seção 6.9 na imagem de teste Testcase 02-12. No canto inferior direito de cada imagem encontra-se uma região ampliada de uma área de interesse do exame que está sendo analisado.

Figura 10.5: Aplicações de todos os filtros discutidos no capítulo 3, com as configurações de parâmetros determinadas na seção 6.9 na imagem de teste Testcase 02-29. No canto inferior direito de cada imagem encontra-se uma região ampliada de uma área de interesse do exame que está sendo analisado.

Figura 10.6: Aplicações de todos os filtros discutidos no capítulo 3, com as configurações de parâmetros determinadas na seção 6.9 na imagem de teste Testcase 02-45. No canto inferior direito de cada imagem encontra-se uma região ampliada de uma área de interesse do exame que está sendo analisado.

Figura 10.7: Aplicações de todos os filtros discutidos no capítulo 3, com as configurações de parâmetros determinadas na seção 6.9 na imagem de teste Testcase 03-29. No canto inferior direito de cada imagem encontra-se uma região ampliada de uma área de interesse do exame que está sendo analisado.

Figura 10.8: Aplicações de todos os filtros discutidos no capítulo 3, com as configurações de parâmetros determinadas na seção 6.9 na imagem de teste Testcase 03-44. No canto inferior direito de cada imagem encontra-se uma região ampliada de uma área de interesse do exame que está sendo analisado.

Figura 10.9: Aplicações de todos os filtros discutidos no capítulo 3, com as configurações de parâmetros determinadas na seção 6.9 na imagem de teste Testcase 03-61. No canto inferior direito de cada imagem encontra-se uma região ampliada de uma área de interesse do exame que está sendo analisado.

Figura 10.10: Aplicações de todos os filtros discutidos no capítulo 3, com as configurações de parâmetros determinadas na seção 6.9 na imagem de teste Testcase 04-38. No canto inferior direito de cada imagem encontra-se uma região ampliada de uma área de interesse do exame que está sendo analisado.

Figura 10.11: Aplicações de todos os filtros discutidos no capítulo 3, com as configurações de parâmetros determinadas na seção 6.9 na imagem de teste Testcase 05-26. No canto inferior direito de cada imagem encontra-se uma região ampliada de uma área de interesse do exame que está sendo analisado.

Figura 10.12: Aplicações de todos os filtros discutidos no capítulo 3, com as configurações de parâmetros determinadas na seção 6.9 na imagem de teste 
Testcase 07-35. No canto inferior direito de cada imagem encontra-se uma região ampliada de uma área de interesse do exame que está sendo analisado.

Figura 10.13: Aplicações de todos os filtros discutidos no capítulo 3, com as configurações de parâmetros determinadas na seção 6.9 na imagem de teste Testcase 13-11. No canto inferior direito de cada imagem encontra-se uma região ampliada de uma área de interesse do exame que está sendo analisado.

Figura 10.14: Aplicações de todos os filtros discutidos no capítulo 3, com as configurações de parâmetros determinadas na seção 6.9 na imagem de teste Testcase 13-28. No canto inferior direito de cada imagem encontra-se uma região ampliada de uma área de interesse do exame que está sendo analisado.

Figura 10.15: Aplicações de todos os filtros discutidos no capítulo 3, com as configurações de parâmetros determinadas na seção 6.9 na imagem de teste Testcase 13-47. No canto inferior direito de cada imagem encontra-se uma região ampliada de uma área de interesse do exame que está sendo analisado.

Figura 10.16: Aplicações de todos os filtros discutidos no capítulo 3, com as configurações de parâmetros determinadas na seção 6.9 na imagem de teste Testcase 14-12. No canto inferior direito de cada imagem encontra-se uma região ampliada de uma área de interesse do exame que está sendo analisado.

Figura 10.17: Aplicações de todos os filtros discutidos no capítulo 3, com as configurações de parâmetros determinadas na seção 6.9 na imagem de teste Testcase 14-36. No canto inferior direito de cada imagem encontra-se uma região ampliada de uma área de interesse do exame que está sendo analisado.

Figura 10.18: Aplicações de todos os filtros discutidos no capítulo 3, com as configurações de parâmetros determinadas na seção 6.9 na imagem de teste Testcase 14-59. No canto inferior direito de cada imagem encontra-se uma região ampliada de uma área de interesse do exame que está sendo analisado.

Figura 11.1 - Na linha superior estão as imagens obtidas pela subtração da imagem Ground Truth Testcase01_26 com a mediana, pixel a pixel, do melhor resultado todos os filtros, enquanto na imagem central está a mesma subtração, mas neste caso somente com o melhor resultado dos filtros PPB_Nakagami, SADCTD, SARBM3D, BLF_L0, DSDN, L0 e FoE_Naka e na imagem à direita está a subtração da imagem ground truth com a imagem original. Na linha inferior encontram-se as imagens medianas respectivas, assim como as imagens de Grouth Truth e Original.

Figura 11.2 - Na linha superior estão as imagens obtidas pela subtração da imagem Ground Truth Testcase01_46 com a mediana, pixel a pixel, do melhor resultado todos os filtros, enquanto na imagem central está a mesma subtração, mas neste caso somente com o melhor resultado dos filtros PPB_Nakagami, SADCTD, SARBM3D, BLF_L0, DSDN, L0 e FoE_Naka e na imagem à direita está a subtração da imagem ground truth com a imagem original. $\mathrm{Na}$ linha inferior encontram-se as imagens medianas respectivas, assim como as imagens de Grouth Truth e Original. 
Figura 11.3 - Na linha superior estão as imagens obtidas pela subtração da imagem Ground Truth Testcase01_62 com a mediana, pixel a pixel, do melhor resultado todos os filtros, enquanto na imagem central está a mesma subtração, mas neste caso somente com o melhor resultado dos filtros PPB_Nakagami, SADCTD, SARBM3D, BLF_L0, DSDN, L0 e FoE_Naka e na imagem à direita está a subtração da imagem ground truth com a imagem original. Na linha inferior encontram-se as imagens medianas respectivas, assim como as imagens de Grouth Truth e Original.

Figura 11.4 - Na linha superior estão as imagens obtidas pela subtração da imagem Ground Truth Testcase02_12 com a mediana, pixel a pixel, do melhor resultado todos os filtros, enquanto na imagem central está a mesma subtração, mas neste caso somente com o melhor resultado dos filtros PPB_Nakagami, SADCTD, SARBM3D, BLF_L0, DSDN, L0 e FoE_Naka e na imagem à direita está a subtração da imagem ground truth com a imagem original. Na linha inferior encontram-se as imagens medianas respectivas, assim como as imagens de Grouth Truth e Original.

Figura 11.5 - Na linha superior estão as imagens obtidas pela subtração da imagem Ground Truth Testcase02_29 com a mediana, pixel a pixel, do melhor resultado todos os filtros, enquanto na imagem central está a mesma subtração, mas neste caso somente com o melhor resultado dos filtros PPB_Nakagami, SADCTD, SARBM3D, BLF_L0, DSDN, L0 e FoE_Naka e na imagem à direita está a subtração da imagem ground truth com a imagem original. Na linha inferior encontram-se as imagens medianas respectivas, assim como as imagens de Grouth Truth e Original.

Figura 11.6 - Na linha superior estão as imagens obtidas pela subtração da imagem Ground Truth Testcase02_45 com a mediana, pixel a pixel, do melhor resultado todos os filtros, enquanto na imagem central está a mesma subtração, mas neste caso somente com o melhor resultado dos filtros PPB_Nakagami, SADCTD, SARBM3D, BLF_L0, DSDN, L0 e FoE_Naka e na imagem à direita está a subtração da imagem ground truth com a imagem original. $\mathrm{Na}$ linha inferior encontram-se as imagens medianas respectivas, assim como as imagens de Grouth Truth e Original.

Figura 11.7 - Na linha superior estão as imagens obtidas pela subtração da imagem Ground Truth Testcase03_29 com a mediana, pixel a pixel, do melhor resultado todos os filtros, enquanto na imagem central está a mesma subtração, mas neste caso somente com o melhor resultado dos filtros PPB_Nakagami, SADCTD, SARBM3D, BLF_L0, DSDN, L0 e FoE_Naka e na imagem à direita está a subtração da imagem ground truth com a imagem original. Na linha inferior encontram-se as imagens medianas respectivas, assim como as imagens de Grouth Truth e Original.

Figura 11.8 - Na linha superior estão as imagens obtidas pela subtração da imagem Ground Truth Testcase03_44 com a mediana, pixel a pixel, do melhor resultado todos os filtros, enquanto na imagem central está a mesma subtração, mas neste caso somente com o melhor resultado dos filtros PPB_Nakagami, SADCTD, SARBM3D, BLF_L0, DSDN, L0 e FoE_Naka e na imagem à direita está a subtração da imagem ground truth com a imagem original. $\mathrm{Na}$ linha inferior encontram-se as imagens medianas respectivas, assim como as imagens de Grouth Truth e Original.

Figura 11.9 - Na linha superior estão as imagens obtidas pela subtração da imagem Ground Truth Testcase03_61 com a mediana, pixel a pixel, do 
melhor resultado todos os filtros, enquanto na imagem central está a mesma subtração, mas neste caso somente com o melhor resultado dos filtros PPB_Nakagami, SADCTD, SARBM3D, BLF_L0, DSDN, L0 e FoE_Naka e na imagem à direita está a subtração da imagem ground truth com a imagem original. $\mathrm{Na}$ linha inferior encontram-se as imagens medianas respectivas, assim como as imagens de Grouth Truth e Original.

Figura 11.10 - Na linha superior estão as imagens obtidas pela subtração da imagem Ground Truth Testcase04_38 com a mediana, pixel a pixel, do melhor resultado todos os filtros, enquanto na imagem central está a mesma subtração, mas neste caso somente com o melhor resultado dos filtros PPB_Nakagami, SADCTD, SARBM3D, BLF_L0, DSDN, L0 e FoE_Naka e na imagem à direita está a subtração da imagem ground truth com a imagem original. Na linha inferior encontram-se as imagens medianas respectivas, assim como as imagens de Grouth Truth e Original.

Figura 11.11 - Na linha superior estão as imagens obtidas pela subtração da imagem Ground Truth Testcase05_26 com a mediana, pixel a pixel, do melhor resultado todos os filtros, enquanto na imagem central está a mesma subtração, mas neste caso somente com o melhor resultado dos filtros PPB_Nakagami, SADCTD, SARBM3D, BLF_L0, DSDN, L0 e FoE_Naka e na imagem à direita está a subtração da imagem ground truth com a imagem original. Na linha inferior encontram-se as imagens medianas respectivas, assim como as imagens de Grouth Truth e Original.

Figura 11.12 - Na linha superior estão as imagens obtidas pela subtração da imagem Ground Truth Testcase07_35 com a mediana, pixel a pixel, do melhor resultado todos os filtros, enquanto na imagem central está a mesma subtração, mas neste caso somente com o melhor resultado dos filtros PPB_Nakagami, SADCTD, SARBM3D, BLF_L0, DSDN, L0 e FoE_Naka e na imagem à direita está a subtração da imagem ground truth com a imagem original. Na linha inferior encontram-se as imagens medianas respectivas, assim como as imagens de Grouth Truth e Original.

Figura 11.13 - Na linha superior estão as imagens obtidas pela subtração da imagem Ground Truth Testcase13_11 com a mediana, pixel a pixel, do melhor resultado todos os filtros, enquanto na imagem central está a mesma subtração, mas neste caso somente com o melhor resultado dos filtros PPB_Nakagami, SADCTD, SARBM3D, BLF_L0, DSDN, L0 e FoE_Naka e na imagem à direita está a subtração da imagem ground truth com a imagem original. Na linha inferior encontram-se as imagens medianas respectivas, assim como as imagens de Grouth Truth e Original.

Figura 11.14 - Na linha superior estão as imagens obtidas pela subtração da imagem Ground Truth Testcase13_28 com a mediana, pixel a pixel, do melhor resultado todos os filtros, enquanto na imagem central está a mesma subtração, mas neste caso somente com o melhor resultado dos filtros PPB_Nakagami, SADCTD, SARBM3D, BLF_L0, DSDN, L0 e FoE_Naka e na imagem à direita está a subtração da imagem ground truth com a imagem original. Na linha inferior encontram-se as imagens medianas respectivas, assim como as imagens de Grouth Truth e Original.

Figura 11.15 - Na linha superior estão as imagens obtidas pela subtração da imagem Ground Truth Testcase13_47 com a mediana, pixel a pixel, do melhor resultado todos os filtros, enquanto na imagem central está a mesma subtração, mas neste caso somente com o melhor resultado dos filtros 
PPB_Nakagami, SADCTD, SARBM3D, BLF_L0, DSDN, L0 e FoE_Naka e na imagem à direita está a subtração da imagem ground truth com a imagem original. Na linha inferior encontram-se as imagens medianas respectivas, assim como as imagens de Grouth Truth e Original.

Figura 11.16 - Na linha superior estão as imagens obtidas pela subtração da imagem Ground Truth Testcase14_12 com a mediana, pixel a pixel, do melhor resultado todos os filtros, enquanto na imagem central está a mesma subtração, mas neste caso somente com o melhor resultado dos filtros PPB_Nakagami, SADCTD, SARBM3D, BLF_L0, DSDN, L0 e FoE_Naka e na imagem à direita está a subtração da imagem ground truth com a imagem original. Na linha inferior encontram-se as imagens medianas respectivas, assim como as imagens de Grouth Truth e Original.

Figura 11.17 - Na linha superior estão as imagens obtidas pela subtração da imagem Ground Truth Testcase14_36 com a mediana, pixel a pixel, do melhor resultado todos os filtros, enquanto na imagem central está a mesma subtração, mas neste caso somente com o melhor resultado dos filtros PPB_Nakagami, SADCTD, SARBM3D, BLF_L0, DSDN, L0 e FoE_Naka e na imagem à direita está a subtração da imagem ground truth com a imagem original. Na linha inferior encontram-se as imagens medianas respectivas, assim como as imagens de Grouth Truth e Original.

Figura 11.18 - Na linha superior estão as imagens obtidas pela subtração da imagem Ground Truth Testcase14_59 com a mediana, pixel a pixel, do melhor resultado todos os filtros, enquanto na imagem central está a mesma subtração, mas neste caso somente com o melhor resultado dos filtros PPB_Nakagami, SADCTD, SARBM3D, BLF_L0, DSDN, L0 e FoE_Naka e na imagem à direita está a subtração da imagem ground truth com a imagem original. Na linha inferior encontram-se as imagens medianas respectivas, assim como as imagens de Grouth Truth e Original. 


\section{Lista de tabelas}

Tabela 3.1: Visão geral dos métodos de filtragem usados e seus respectivos parâmetros. Os números encontrados após o nome de cada filtro são referentes à seção que eles pertencem. ITL (Iteration Length) representa quantas vezes um filtro foi reaplicado, enquant SWS (Sliding Window Size) o tamanho da janela deslizante utilizada.

Tabela 4.1: Limites teóricos e práticos das métricas, bem como seus melhores resultados e o grupo a qual pertencem.

Tabela 4.2: Os pesos adotados para cada métrica para o sistema de avaliação Weighted.

Tabela 6.1: Intervalo de variação de cada parâmetro para cada filtro e o total de variações usadas para cada filtro. ITL (Iteration Length) representa quantas vezes um filtro foi reaplicado, enquant SWS (Sliding Window Size) o tamanho da janela deslizante utilizada.

Tabela 6.2: Valores definidos dos parâmetros de cada filtro de acordo com o método de seleção de centroide dos clusters por Cardinalidade Relativa, com o número total de clusters definido por kSqrt, na métrica de agrupamento Full Reference, na categoria Light.

Tabela 8.1: Somatório dos melhores resultados de cada filtro para cada métrica em cada teste normalizado para o intervalo [0,1]. Em negrito estão os 3 melhores resultados de cada métrica.

Tabela 8.2: Resultado da aplicação das métricas de agrupamento sobre os dados vistos na Tabela 8.1. Em negrito estão os 3 melhores resultados de cada métrica.

Tabela 8.3: Métricas calculadas para todas as imagens de teste do filtro PPB_Nakagami e normalizadas para o intervalo [0.1] segundo a discussão apresentada no capítulo 4. Em negrito estão os 3 melhores resultados de cada métrica, enquanto em itálico e sublinhado estão os resultados de média e mediana com valores acima de 0.8 .

Tabela 8.4: Métricas calculadas para todas as imagens de teste do filtro SADCTD e normalizadas para o intervalo [0.1] segundo a discussão apresentada no capítulo 4. Em negrito estão os 3 melhores resultados de cada métrica, enquanto em itálico e sublinhado estão os resultados de média e mediana com valores acima de 0.8 .

Tabela 8.5: Métricas calculadas para todas as imagens de teste do filtro SARBM3D e normalizadas para o intervalo [0.1] segundo a discussão apresentada no capítulo 4. Em negrito estão os 3 melhores resultados de cada métrica, enquanto em itálico e sublinhado estão os resultados de média e mediana com valores acima de 0.8 . 


\section{Introdução}

O imageamento medico por ultrassom é uma técnica que se tornou mais popular que qualquer outra técnica de aquisição de imagens médicas por ser um procedimento mais acessível, menos custoso, seguro, de fácil manuseio, nãoinvasivo, produz imagens em tempo real e por ser capaz de gerar imagens acuradas sem o uso de radiação, ao contrário das Ressonâncias Magnéticas Induzidas ou Tomografias Computadorizadas. Entretanto, este tipo de imagem é degradada por um artefato "speckle", que é o resultado da soma coerente construtiva e destrutiva de ecos de ultrassom, afetando a qualidade visual do exame e consequentemente diagnósticos e métodos de pós-processamento.

O Sistema de imagem por Ultrassom é baseado na captura de diferentes pulsos sônicos dispersados pela superfície entre dois tecidos com diferentes características acústicas, onde uma parte da onda incidente é refletida na direção da sonda, formando ecos. Entretanto, em conjunto com esses estão os ecos retro dispersados, originário das estruturas microscópicas do tecido, sendo esses os dois principais tipos de ecos existentes em exames de ultrassom: a dispersão difusa, gerando manchas (speckle) na imagem e dispersão coerente, produzindo traços claros e escuros. A dispersão difusa ocorre quando existe um grande número de espalhamento na região de atuação da sonda, enquanto a dispersão coerente é resultante de espalhamentos que se encontram em fase [1].

Considerado um ruído por ser responsável pela diminuição da qualidade visual do exame de ultrassom, a remoção do speckle é ainda alvo de diversos estudos, que visam não só melhorar a acurácia de detecção de objetos de interesse, seja por parte dos profissionais da área médica ou por métodos de pósprocessamento, quanto preservar elementos de diagnóstico importantes. A suavização deste ruído e a preservação de arestas são geralmente tidos como problemas opostos, sendo assim necessário encontrar um meio-termo entre ambos para que um bom resultado seja obtido.

Outro fator importante a ser analisado vem do fato dos métodos tradicionais de avaliação de desempenho dos filtros necessitarem de imagens ground truth de referência para calcular a "distância" que a imagem filtrada ficou desta imagem sem ruído. No caso do ultrassom, entretanto, a imagem de referência não existe, sendo assim necessário simular esse caso, seja aplicando um modelo de ruído speckle a uma imagem limpa e então filtrando-a, ou preparando a imagem de referência de forma manual por um especialista ou ainda procurando por técnicas de análise de desempenho que não necessitem de imagens ground truth para avaliação.

O próprio comportamento das avaliações de desempenho é um caso de estudo importante dada a baixa qualidade visual das imagens de ultrassom e da falta de fronteiras bem definidas. Além disso, existem diversas formas distintas de avaliação, portanto uma forma de determinar quais delas de fato são relevantes para esta comparação também precisou ser analisada.

Por fim, cada filtro possui suas respectivas características e, portanto, cada um utiliza um conjunto de parâmetros próprios. Uma vez com o método de avaliação de imagens definido, os parâmetros de cada filtro também podem ser determinados.

Neste trabalho, diversos filtros foram levados em consideração, visando suprimir o problema do ruído speckle enquanto ainda mantendo as características fundamentais da imagem original. A avaliação dos resultados de cada filtro foi feita 
pelo uso de diversas métricas, que por sua vez também foram avaliadas para que um grupo de métricas ótimas fosse encontrado. A análise das métricas exigiu que diversos métodos fossem estudados e testados para que enfim um escopo de testes completo fosse feito, contemplando todas as etapas descritas anteriormente.

\subsection{Motivação}

Por ser fortemente correlacionado com as microestruturas do tecido colidido, o padrão de speckle de uma determinada área será idealmente único e, portanto, identificável, fazendo com que o speckle possa ser tratado como uma fonte fundamental de informação. Essa caracterização do ruído leva à classificação do tecido em si, sendo este um passo de essencial importância para áreas como segmentação, reconstrução, análise, reconhecimento, medidas quantitativas e qualitativas, sem contar na ajuda da avaliação em casos como elastografias, eco cardiogramas, entre outros. Portanto, a suavização ou remoção do ruído speckle, ou despeckling, mantendo ou ressaltando suas informações intrínsecas é fundamental para um avanço nesses campos de estudos.

Além disso, percebemos que a vasta maioria dos estudos envolvendo filtros de despeckling não aborda a maneira como os parâmetros destes devam ser selecionados, com a comparação entre filtros ficando, portanto, prejudicada ao ter somente uma ou poucas performances de um mesmo filtro sendo testado.

O tema de comparação de filtros para imagens de ultrassom é um tanto delicado visto que não existem imagens ground-truth para serem levadas em consideração, ou seja, sem uma base sólida a qual diversos filtros possam ser comparados, portanto esta questão também nos motivou a estudar sobre o assunto.

Como a própria avaliação dos filtros não é trivial, a busca pelo conjunto de métodos que pudesse analisar, sem supervisão e de forma acurada, o desempenho de cada imagem, também nos motivou a realizar este estudo.

Tendo como base a primeira versão do estudo feito por Loizou[2] em 2008, notamos que os filtros usados poderiam ser considerados defasados em alguns casos, já existindo novos algoritmos com características diferentes que possivelmente gerariam resultados melhores. Além disso, Loizou, neste estudo, não explicitou detalhadamente o procedimento utilizado para a seleção dos parâmetros de cada filtro.

\subsection{Objetivo}

O objetivo do trabalho será desenvolver um framework responsável por avaliar o desempenho de um filtro ao mesmo tempo que varia seus parâmetros, para assim encontrar uma configuração que melhor suavize ou remova o ruído speckle enquanto mantém as principais características do exame de ultrassom.

Uma vez que a própria questão de avaliação de desempenho é um campo de estudo à parte em imagens médicas, este também será levado em consideração aqui, ao procurarmos uma metodologia consistente de comparação para enfim poder melhor avaliar um resultado em detrimento de outros.

\subsection{Estrutura}

A estrutura deste trabalho dará da seguinte forma: No capítulo 2 é feita uma revisão de conceitos e dos trabalhos relacionados; no capítulo 3 serão apresentados 
os filtros estudados, com suas formulações matemáticas ou algoritmos; o capítulo 4 contém as fórmulas e algoritmos usados para medir o desempenho de cada filtro, bem como suas categorizações e tipos de pontuações abordados; no capítulo 5 a metodologia de comparação final será introduzida, sendo também responsável por detalhar as escolhas dos parâmetros para cada filtro; no capítulo 6 abordaremos a comparação e avaliação geral de dados encontrados ao longo desta pesquisa; no capítulo 7 estarão as conclusões encontradas neste projeto e possíveis trabalhos futuros e finalmente o Anexo A é apresentado com figuras contendo os resultados obtidos. 


\section{Fundamentação Teórica}

\subsection{Imagens por Ultrassom}

Ultrassom é a onda sonora com frequência que excede os $20 \mathrm{kHz}$, onde sua interação com tecidos está sujeita às leis de geometria óptica, incluindo reflexão, refração, espalhamento, difração, interferência e absorção, sendo que exceto pela interferência, todas as outras interações reduzem a intensidade do sinal de ultrassom[3]. Esse processo de perda de energia é conhecido como atenuação, que aumenta gradativamente com a distância percorrida pelo eco e sua frequência. Como uma frequência maior do ultrassom resulta em uma maior absorção por parte dos tecidos, a consequência é uma perda na profundidade da visualização. Um exemplo deste problema se encontra ao realizar um exame de toda uma estrutura, como um órgão ou um feto, em que apesar de uma alta frequência garantir um bom resultado para a parte da estrutura que está mais perto do emissor de ultrassom, a parte mais distante ficará com uma qualidade deteriorada, portanto um compromisso precisa ser feito.

O modelo mais popular usado na literatura para explicar como funciona a aquisição de dados por ultrassom é ilustrado na Figura 2.1, onde o tecido pode ser modelado como um meio que absorve as ondas sonoras e as espalha. Esse espalhamento advém da heterogeneidade do tecido e de microestruturas, menores em tamanho que o comprimento da onda de ultrassom, tais como o parênquima, onde existem mudanças na impedância acústica em nível microscópico dentro do tecido.

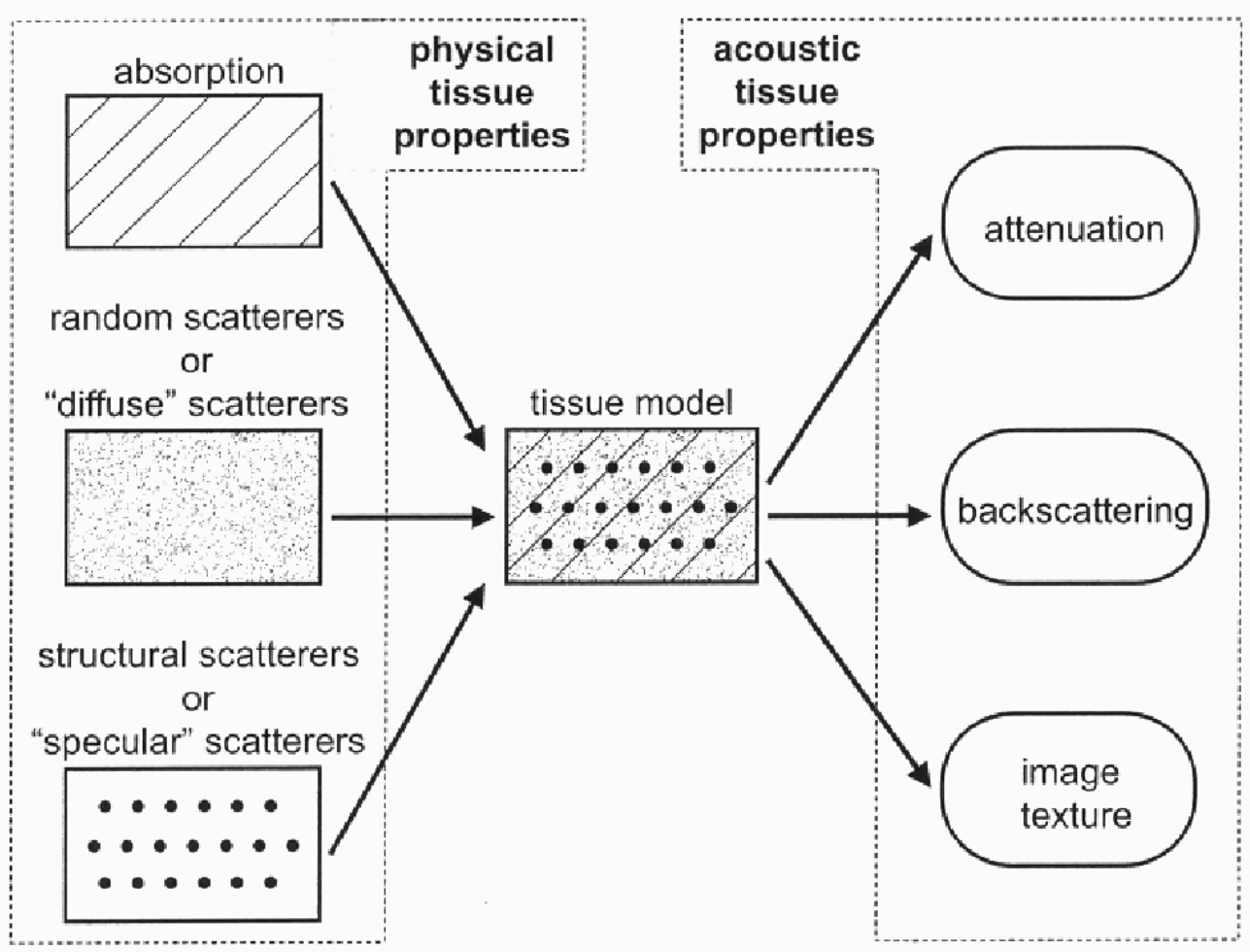

Figura 2.1: O modelo usual de tecido em imagens de ultrassom[2]. 
A Figura 2.2 ilustra todo o procedimento de espalhamento. Na Figura 2.2.a temos um transdutor emitindo um raio de ultrassom, que colide em quatro pontos diferentes do meio encontrado, gerando assim quatro espalhamentos distintos. Esses espalhamentos geram ondas esféricas que chegarão ao transdutor em momentos levemente diferentes após sua emissão. Ao ter o sinal recebido, o transdutor produz um sinal de radiofrequência (RF) que é a soma algébrica das pressões sonoras instantâneas originadas das ondas refletidas, gerando a visualização obtida na Figura 2.2.b. As diferenças de profundidade são menores que o comprimento do pulso do transdutor, sendo essa a causa básica da geração da textura do tecido, com um padrão chamado de speckle. Nesse exemplo ainda temos que a textura oriunda desse padrão de speckle não é de fato a estrutura histológica do tecido, mas um padrão de interferência determinado principalmente pelas características do raio de ultrassom.
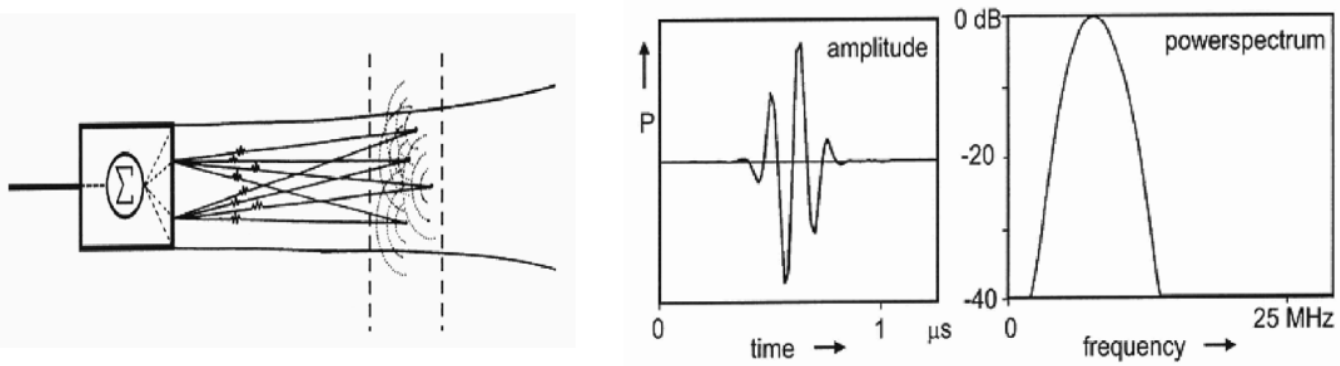

Figura 2.2: (a) $\mathrm{O}$ espalhamento do raio de ultrassom. (b) Um pulso no tempo e no domínio da frequência [2].

O sinal de RF por conter a informação completa do exame, possui uma resolução maior e não rasterizada, sendo necessário um grande custo computacional e espaço de armazenamento para processá-lo[2]. Entretanto, sua vantagem é que ele não é afetado por nenhum passo de pós-processamento como compressão logarítmica e/ou aplicação de filtros, que mudam as características intrínsecas do ruído a fim de melhorar o aspecto visual do exame, mantendo ainda suas propriedades estatísticas originais, que mais facilmente se adequam a modelos de distribuição, como o ruído speckle [4]. Geralmente os passos de pós-processamento são desconhecidos para o usuário e podem em muitos casos resultar na modificação da distribuição dos níveis de cinza, como por exemplo obtendo histogramas de gaussianas, que não são tipicamente observados no sinal de RF.

A conversão dos dados em RF para Coordenadas Cartesianas faz com que a informação seja rasterizada, ficando consequentemente com uma resolução menor e, conforme visto antes, geralmente uma compressão/filtragem é feita com o intuito de melhorar a qualidade visual do exame ao custo da perda de confiabilidade. Esses dados passam a ser mais fáceis de serem tratados e, portanto, este é o formato usualmente abordado na literatura e o que será abordado ao longo deste trabalho.

$\mathrm{O}$ dado em Coordenadas Cartesianas pode existir em diferentes formatos, mas os principais tipos de são os Modos A e B, apesar do Modo M, ultrassom duplex, codificado em cores e power Doppler, entre outros, também serem usados. Os modos A, B e M podem ser observados na Figura 2.3. 
O Modo A se refere ao tipo de escaneamento por amplitude, sendo mantido por interesses históricos, onde os ecos são observados como ondas representadas em linhas, com reflexões intensas descrevendo um aumento na amplitude do sinal. Já o Modo B, que será usado por este trabalho, é referente ao tipo de escaneamento por brilho (brightness), onde a amplitude dos ecos é representada como pontos (pixels) em uma imagem 2D em tons de cinza. Quando os modos A ou B são observados ao longo do tempo, temos o modo $\mathrm{M}$, sendo este, portanto análogo a gravar um vídeo de ultrassom e mais usado para determinar a velocidade de certas estruturas.
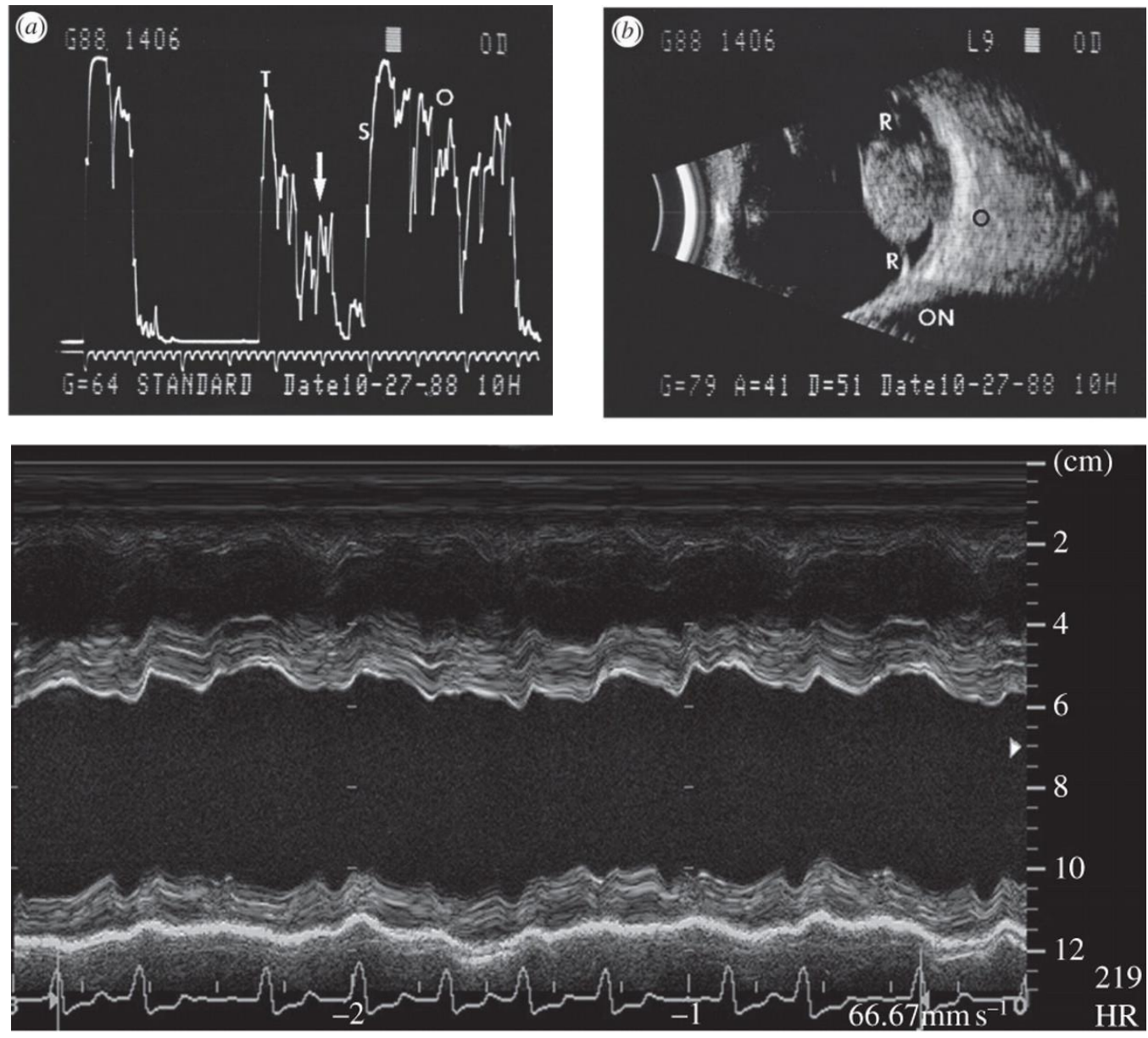

\section{Figura 2.3: Da esquerda para a direita: Ultrassom nos modos A, B e M (embaixo) [5].}

\subsection{Ruído Speckle}

O speckle é um tipo de ruído multiplicativo, fortemente correlacionado e com estatísticas não Gaussianas, sendo assim diretamente proporcional aos níveis de cinza em sua área de atuação e naturalmente dependente dos dados da imagem, podendo ainda ser responsável por reduzir o contraste do exame, adicionar detalhes borrados e produzir pseudo-características, de modo que métodos tradicionais de análise de imagem não são ótimos para exames de ultrassom[6] uma vez que eles usualmente seguem estatísticas Gaussianas para tratamento e não levam em consideração que o ruído pode acarretar informação essencial à imagem. 
O tamanho do speckle varia de acordo com a distância do transdutor, de forma que ao escanear um objeto duas vezes sob as mesmas condições, o padrão speckle será o mesmo, ou seja, apesar de ser um ruído aleatório, esta aleatoriedade não é a mesma no sentido de um ruído elétrico, uma vez também que o próprio speckle não é um ruído no sentido típico da engenharia por ele também conter informação relevante.

Por sua natureza complexa, diversos modelos de distribuição que o caracterizam foram propostos: Modelos de Rayleigh e Ricianos foram originalmente apresentados, onde apresentaram que o speckle totalmente desenvolvido seguia uma função de distribuição de probabilidade (Probability Density Function, PDF) de Rayleigh[7][8]. Conforme as componentes coerentes cresceram, o sinal começou a se comportar como um PDF Riciano[9]. Entretanto foi descoberto que apesar da distribuição de Rayleigh ser bem usada para reflexões advindas de sangue, ela é falha em estruturas complexas como o tecido do miocárdio[10]. Portanto modelos mais genéricos foram propostos como o Nakagami[11], Gamma Generalizado[12], k[13], k Generalizado e Distribuição kHomodyned[14][15], responsáveis por uma melhor descrição do speckle ao custo de uma formulação mais complexa.

Como pode-se perceber, o ruído speckle além de não ser um ruído comum, não existe uma formulação fechada para o seu comportamento ou maneiras simples de reproduzi-lo ou suprimi-lo, razão pelo qual ainda hoje ele é um tema recorrente em estudos.

\subsection{Filtros}

A redução do ruído speckle e a preservação de arestas podem ser vistos como ideias opostas, necessitando assim de um compromisso entre ambos para melhorar a qualidade visual dos elementos relevantes da imagem, enquanto ainda diminui a variância em regiões homogêneas e mantém regiões distintas bem definidas, então para atingir um bom desempenho nesse âmbito, o filtro deve se adaptar às descontinuidades na imagem.

Por não ser um problema trivial, existem diversos estudos acerca da redução ou supressão do ruído speckle. Lee[16], Frost et al.[17] e Kuan et al[18] foram considerados o estado da arte por anos, utilizando estatísticas locais em uma janela deslizante para suavizar a imagem. Autores abordaram diferentes técnicas na construção de seus filtros, como difusão anisotrópica[19]; homomorfismo[20], alterando as características do ruído com o intuito de poder considera-lo aditivo ao invés de multiplicativo; estatísticas não locais (nonlocal means)[21], aproveitando do fato de regiões (patches) se repetirem ao longo da imagem, sendo assim tratados como informação redundante e podendo ser filtrados de maneira similar ou ainda possibilitando uma filtragem 3D dos patches empilhados[22]; filtros baseados em imagens de satélite (Synthetic Aperture Radar, SAR)[23], com a formulação do ruído sendo semelhante ao speckle;

Dada a vasta formulação das soluções existentes, consequentemente várias categorizações distintas são usadas para classificá-las. Os filtros abordados neste trabalho seguem quatro classificações, que foram escolhidas por sua simplicidade: Filtros lineares; não lineares; por difusão; e outros. Outras classificações incluem Wavelet Shrinkage, SAR, Geometric, entre outras, que não foram abordadas por resultarem em categorias de um único filtro ou terem filtros que poderiam participar de mais de uma classificação. 
Os filtros lineares reduzem o ruído em áreas homogêneas, apesar de normalmente suavizar em excesso regiões heterogêneas ao borrar contornos e arestas na imagem. Os filtros não lineares utilizam métodos que não necessariamente levam em consideração toda a informação presente em uma janela de atuação, geralmente atingindo resultados melhores que os filtros lineares.

Nos filtros por difusão, a suavização depende das arestas da imagem e suas direções, melhorando o contraste da imagem enquanto mantém as arestas. Os filtros restantes concentram técnicas diversas que muitas vezes não foram desenvolvidas pensando no caso do ruído speckle do ultrassom, como o L0 e o PPB, e em alguns casos alteram drasticamente o formato do histograma da imagem de teste, precisando assim de cautela ao serem usados.

As formulações e descrições mais detalhadas destes filtros serão apresentadas no capítulo 3.

\subsection{Métricas}

Visto que um mesmo filtro tem diferentes configurações e diversos filtros são usados em várias imagens de teste, é importante encontrar um método para avaliar automaticamente seu desempenho, pontuando positivamente os que conseguirem atingir uma boa suavização e preservação de arestas e eliminando os que borrarem as fronteiras ou mantiverem uma alta variância em regiões homogêneas. Para atingir esta finalidade, diversas técnicas foram estudadas, analisando diferentes características da imagem.

As métricas podem ser divididas em três grupos diferentes: as de referência completa, comparando a imagem filtrada com um ground truth, as de referência reduzida, comparando o resultado com a imagem ruidosa de entrada e as sem referência, analisando somente a imagem filtrada. Tipicamente o grupo de métricas de referência completa é o mais usado na literatura, apesar dos outros dois grupos possibilitarem o uso de métodos não supervisionados de filtragem ao continuar reaplicando o filtro até que uma pontuação menor nessas métricas seja obtida.

As descrições e fórmulas de cada métrica abordada será vista no capítulo 4.

\subsection{Avaliações}

Dado que diversas métricas serão analisadas, é importante saber dizer quais de fato avaliam melhor as imagens com menos ruído e com melhor definição de fronteira. Para que este objetivo fosse cumprido, outras formas de avaliação são necessárias.

Os resultados de cada filtro podem ser visualizados em vários gráficos $2 \mathrm{D}$ no formato $\mathrm{P}_{\mathrm{i}} \mathrm{XR}$, onde $\mathrm{P}_{\mathrm{i}}$ representa os i-ésimo parâmetro de um mesmo filtro e $\mathrm{R}_{\mathrm{j}} \mathrm{O}$ j-ésimo conjunto de métricas usado, sendo assim necessário um gráfico para cara parâmetro por conjunto de métrica.

Como um bom resultado de filtro será aquele que consistentemente possuir os melhores resultados no maior número de métricas possível, uma maneira de avaliar o desempenho das métricas é criando uma nuvem de pontos $\mathrm{N}$ dimensional e procurando aglomerar aqueles resultados que mais se assemelham entre si. Nesta nuvem, cada ponto exprime um dos resultados obtidos pelas variações de parâmetros de um mesmo filtro e cada parâmetro e métrica representam uma nova 
dimensão. Existem diversos modelos de algoritmos responsáveis pela aglomeração dos dados, como os de Conectividade, Distribuição, Densidade, entre outros[24].

Mesmo após conseguir aglomerar os resultados obtidos, ainda é preciso determinar qual conjunto representa o melhor resultado, que será discutido na seção 6.3 . 


\section{Filtragem 2D}

A performance de vários procedimentos de imagem, como segmentação, detecção de características, classificação, reconstrução $3 \mathrm{D}$, entre outros, são dependentes da qualidade da imagem de entrada, que devem proporcionar dados fidedignos e informações de fácil acesso. Além disso, a existência de uma frequência maior de regiões homogêneas e de regiões homogêneas maiores diminuem o custo computacional destes algoritmos enquanto aumentam sua robustez e acurácia.

A filtragem 2D em imagens de ultrassom tem como objetivo primário a redução do ruído speckle inerente aos próprios exames enquanto ainda visa criar áreas homogêneas onde possível, mantendo ou realçando fronteiras e assim garantindo que as características físicas da estrutura observada pelo exame se mantenham fiel à realidade.
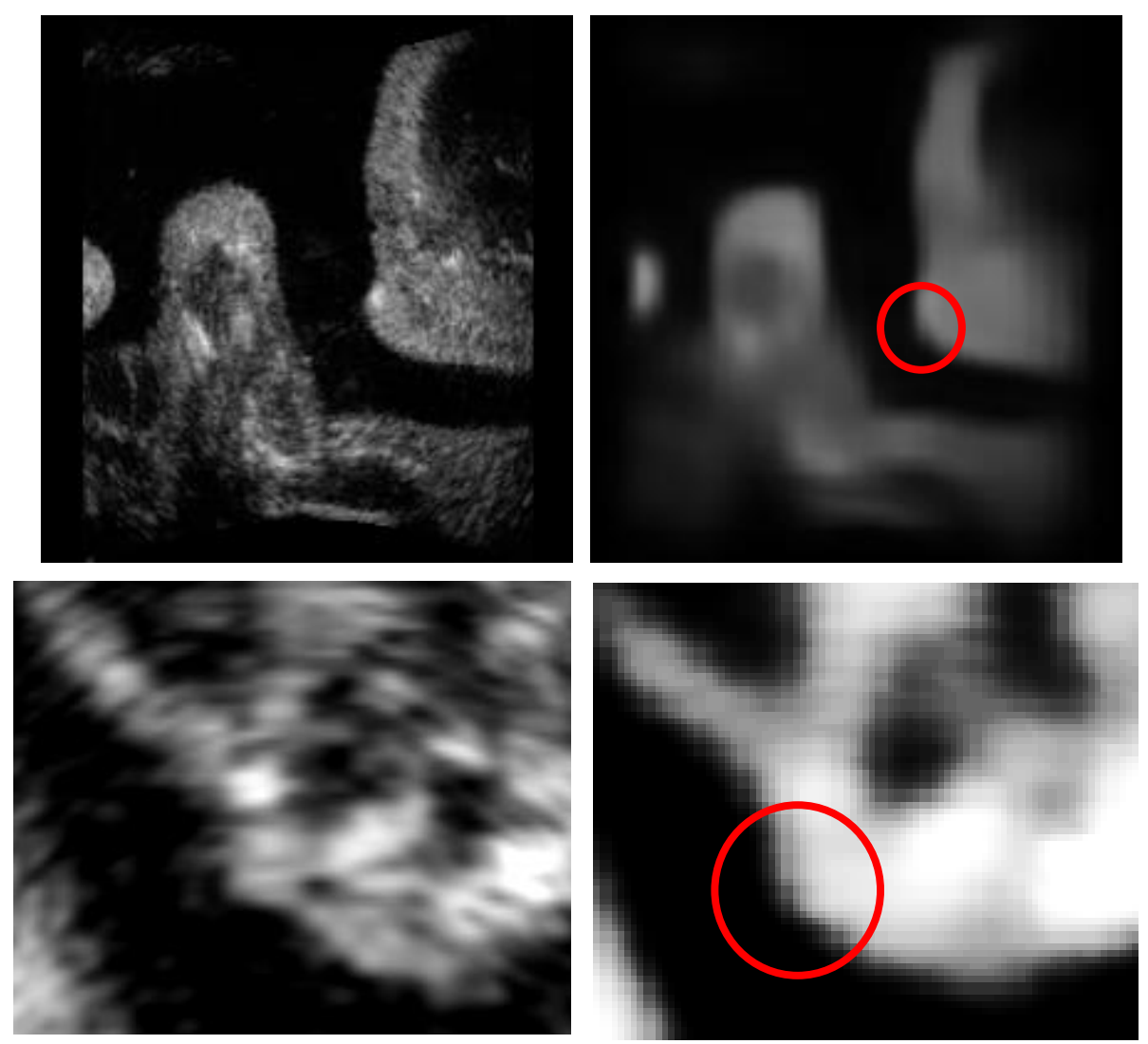

Figura 3.1: Exemplo de filtros que atendem às características desejáveis mas violam os requisitos fundamentais. À esquerda estão as imagens originais e à direita suas versões filtradas, respectivamente. Em ambas as imagens filtradas é possível observar como regiões mais homogêneas foram criadas, entretanto, as estruturas circuladas em vermelho foram deformadas a ponto de perderem o significado que tinham antes. No caso as fronteiras foram drasticamente borradas a ponto de os exames aparentarem ter uma deformação física inexistente no exame original.

Sendo um campo de pesquisa ativo há décadas, o tópico de filtragem de ruído speckle recebe a cada ano propostas de algoritmos cada vez mais eficientes tanto em suavização quanto em custo computacional. Também na categoria de ruído 
speckle encontram-se os filtros para imagens de satélite, que apesar de não serem frequentemente mencionados nos estudos relacionados a ultrassom, podem igualmente apresentar resultados interessantes, mesmo apesar de não levarem em consideração as características próprias do ultrassom. Portanto para poder escolher $\mathrm{o}(\mathrm{s})$ melhor(es) filtro(s), diversos métodos foram testados.

$\mathrm{O}$ filtro elencado deve obedecer duas diretivas principais e tentar seguir outros dois pontos importantes sempre que possível:

- Requisitos Fundamentais

○ Preservar/realçar fronteiras

- Preservar características importantes do exame

- Características Desejáveis

- Criar áreas homogêneas

- Remover o ruído speckle.

Esses quesitos foram adotados visando favorecer os métodos de pósprocessamento já discutidos enquanto ainda melhorando a qualidade visual do exame. Caso as fronteiras ou características do exame não fossem preservados ou realçados, o resultado de uma possível etapa de pós-processamento seria equivocado, podendo apresentar sintomas e/ou anomalias não presentes na estrutura analisada.
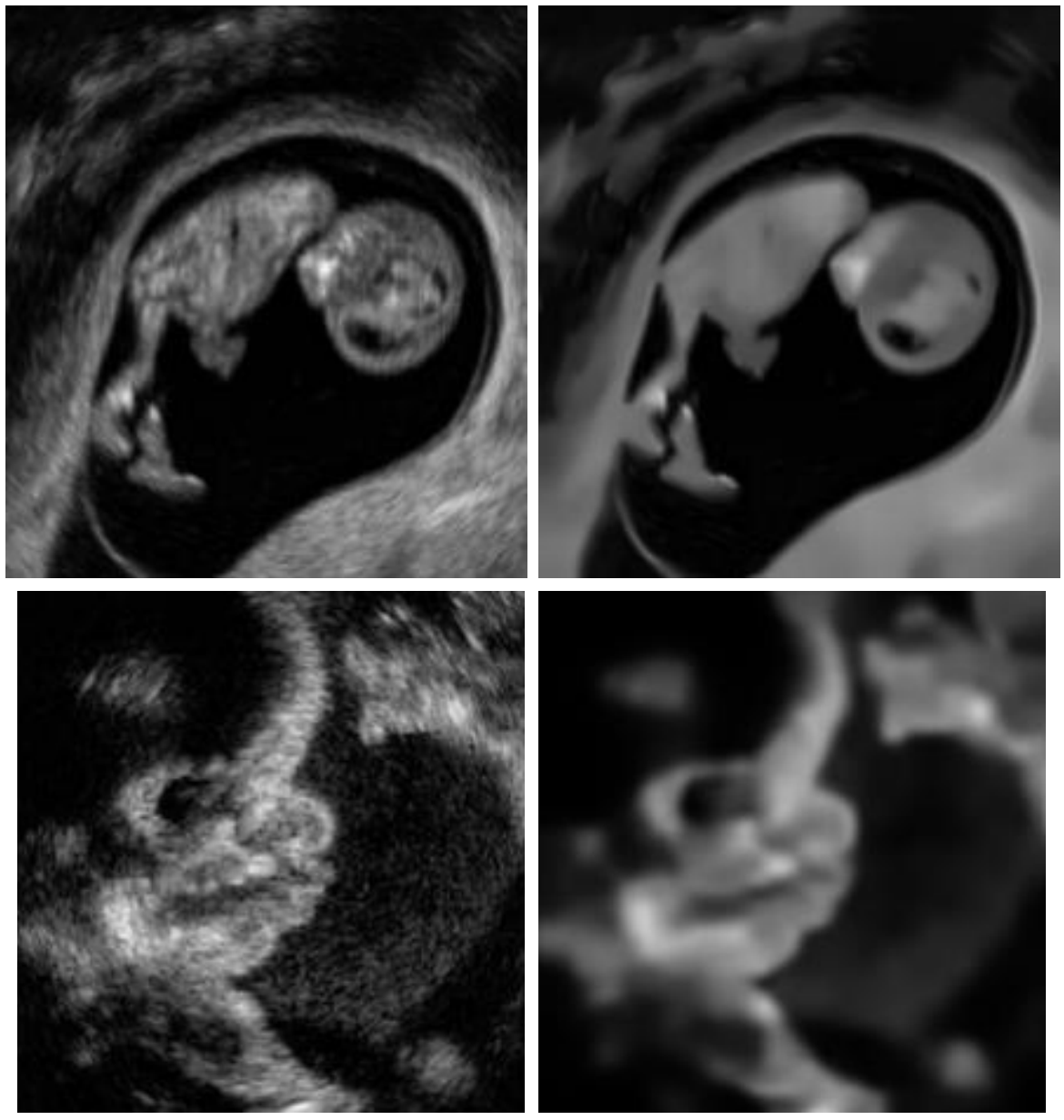

Figura 3.2 - Exemplo de filtros que atendem a ambos os requisitos fundamentais e características desejáveis. À esquerda estão as imagens não processadas e à direita suas versões filtradas, respectivamente. 
Apesar de existirem filtros com alto desempenho nas características desejáveis, como visto na Figura 3.1, muitas vezes estes também acabam por deformar as fronteiras, o que resultaria em uma imagem filtrada pouco fiel com relação a estrutura real observada, portanto estes filtros devem ser considerados com cautela e apenas para filtragens com mais de uma etapa de tratamento de imagem. Assim a Figura 3.2 contém casos em que tanto Requisitos Fundamentais quanto Características Desejáveis são atendidas.

Para melhor compreender como atender aos Requisitos Fundamentais e Características Desejáveis, 23 filtros foram amplamente testados, em que nas fórmulas apresentadas a seguir, $g$ corresponde à imagem ruidosa de entrada, enquanto $f$ representa a imagem filtrada. Os parâmetros de suas respectivas implementações são apresentados na Tabela 3.1.

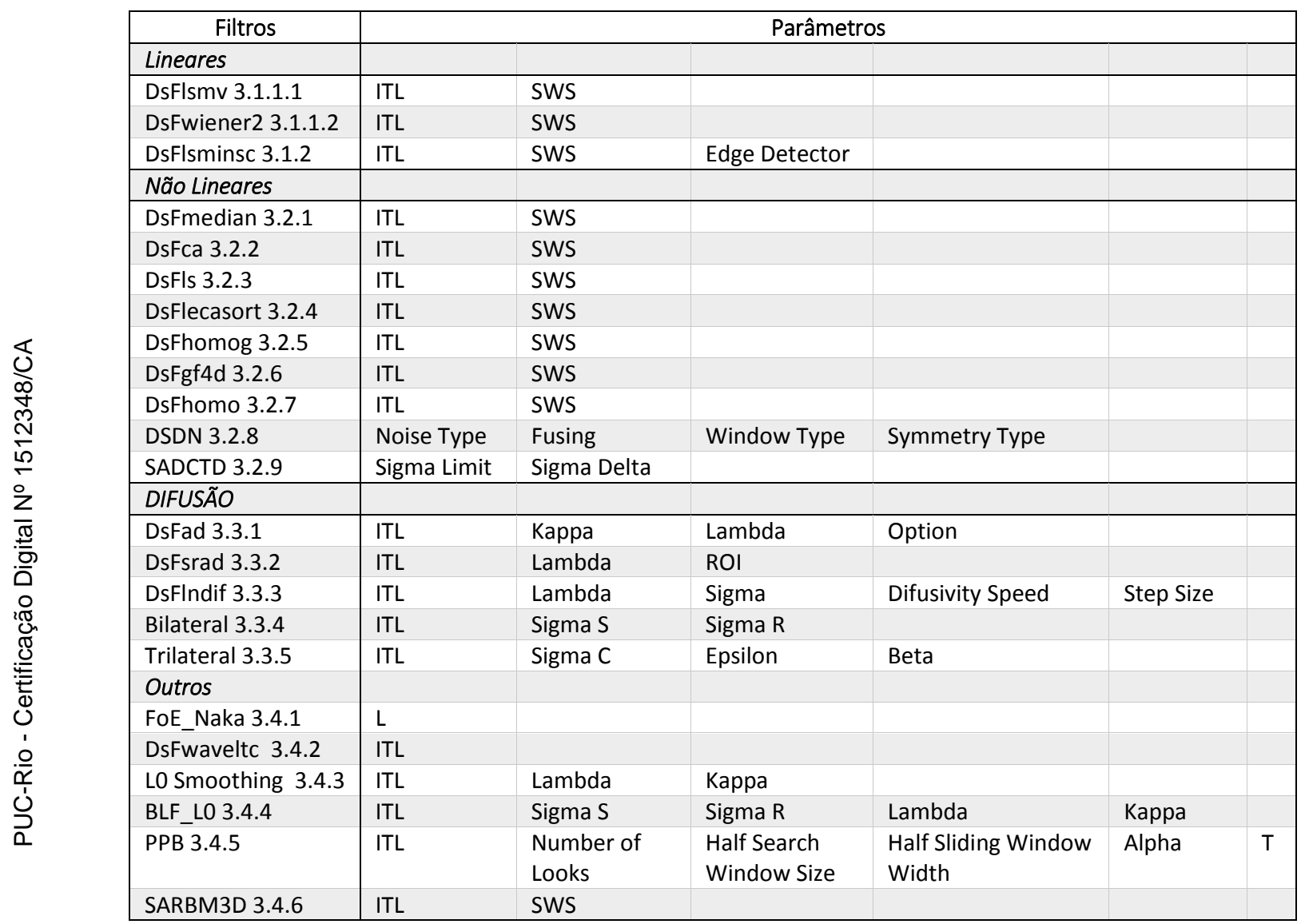

Tabela 3.1: Visão geral dos métodos de filtragem usados e seus respectivos parâmetros. Os números encontrados após o nome de cada filtro são referentes à seção que eles pertencem. ITL (Iteration Length) representa quantas vezes um filtro foi reaplicado, enquant SWS (Sliding Window Size) o tamanho da janela deslizante utilizada.

Esses filtros foram escolhidos por terem suas implementações disponíveis para o ambiente Matlab, alguns já tendo sido avaliados em outros estudos, como os filtros DsF, apresentados por Loizou, et al[2]. Outros, como o L0 Smoothing, Trilateral e o PPB_Nakagami, foram escolhidos pela qualidade da homogeneização apresentada por seus autores, enquanto a seleção de um terceiro grupo de filtros foi feita por estes terem sido bem sucedidos ao tentar solucionar o Additive White Gaussian Noise (AWGN), como o DSDN e o SADCTD, ou o ruído presente em imagens de satélite (SAR), como o SARBM3D e FoE_Naka. 
Os filtros podem ser agrupados em quatro categorias distintas:

1. Filtros Lineares

2. Filtros Não Lineares

3. Filtros de Difusão

4. Outros

\subsection{Filtros lineares}

São filtros que criam pesos baseados em estatísticas locais de uma sliding window (janela deslizante), assumindo que o ruído speckle é multiplicativo.

\subsubsection{Filtros estatísticos de primeira ordem}

Os filtros que utilizam estatísticas de primeira ordem, como variância, média da vizinhança, dispersão, curtose, etc., podem ser descritos usando a seguinte equação:

$f_{i, j}=\bar{g}+k_{i, j}\left(g_{i, j}-\bar{g}\right)$
$\sigma_{n}^{2}=\sum_{i=1}^{p} \sigma_{p}^{2} / \bar{g}_{p}=$ variância do ruído em toda a imagem

$$
f_{i, j}=\bar{g}+k_{i, j}\left(g_{i, j}-\bar{g}\right)
$$

$\sigma_{p}^{2}=$ variância na janela corrente

$\bar{g}_{p}=$ média do ruído na janela selecionada

$p=$ índice usado para iterar sobre todas as janelas em toda a imagem

$f_{i, j}=$ estimativa de valor do pixel sem ruído

$g_{i, j}=$ valor original do pixel (ruidoso) em uma janela

$\bar{g}=$ valor médico local da região de $N_{1} \times N_{2}$ em torno de e incluindo o pixel $g_{i, j}$

$k_{i, j}=$ função que representa a estatística local em uma janela

Em que a função que representa a estatística local de uma janela pode ser derivado de diferentes formas. As duas formas testadas neste trabalho são apresentadas a seguir.

\subsubsection{Mean and variance local statistics Filter (DsFlsmv)}

Usa a seguinte equação[16] para filtrar cada pixel da imagem de entrada

$$
k_{i, j}=\frac{1-\bar{g}^{2} \sigma^{2}}{\sigma^{2}\left(1+\sigma_{n}^{2}\right)}
$$

$\bar{g}_{p}=$ média do ruído na janela selecionada

$\sigma_{n}^{2}=$ variância do ruído em toda a imagem, como visto em 3.1.1

$\sigma_{p}^{2}=$ variância na janela corrente

\subsubsection{Wiener Despeckle Filter (DsFwiener2)}

Usa o método de Wiener[16] adaptativo pixel a pixel, suavizando a imagem baseado no cálculo da variância local.

$$
k_{i, j}=\frac{\sigma^{2}-\sigma_{n}^{2}}{\sigma^{2}}
$$


$\sigma_{n}^{2}=$ variância do ruído em toda a imagem, como visto em 3.1 .1

$\sigma_{p}^{2}=$ variância na janela corrente

\subsubsection{Minimum speckle index homogeneous Mask Area Filtering (DsFlsminsc)}

Opera em um Kernel de vizinhança quadrado procurando pela zona mais homogênea em uma máscara de subset[25]. O pixel do meio do kernel de vizinhança é substituído pela média do nível de cinza da máscara com o menor índice speckle C. A janela com o menor $\mathrm{C}$ é a semi-janela mais homogênea, que provavelmente não contém nenhuma borda.

$$
C=\frac{\sigma_{p}^{2}}{\bar{g}_{p}}
$$

$\bar{g}_{p}=$ média do ruído na janela selecionada

$\sigma_{p}^{2}=$ variância na janela corrente

\subsection{Filtros não lineares}

São os filtros baseados em métodos não lineares em uma sliding window.

\subsubsection{Hybrid Median Filtering (DsFmedian)}

É um filtro de mediana híbrido[26], usando em uma sliding window o seguinte kernel, como exemplo:

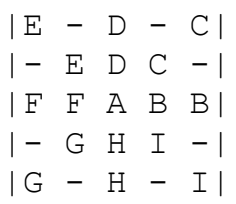

Para então substituir o pixel A, do meio do kernel, por mediana(A, mediana(A,B,D,F,H), mediana(A,C,E,G,I)), segundo o autor, apesar do filtro exigir uma grande carga computacional, ele preserva melhor as arestas que um filtro comum de mediana. Vale ressaltar que este kernel pode ser estendido para quaisquer dimensões quadradas e possui tamanho mínimo $3 \times 3$.

\subsubsection{Linear Scaling of the Gray-Levels Filter (DsFca)}

Aplica uma escala linear aos níveis de cinza da sliding window, computando a média de todos os pixels cuja diferença para o pixel do meio da sliding window é menor ou igual a um threshold $\vartheta$ e atribuindo esse valor ao pixel do meio segundo a seguinte fórmula[27]:

$\alpha=[0,1]$

$$
\vartheta=\alpha * g_{\max }
$$

$g_{\text {max }}=$ pixel de maior valor da sliding window 


\subsubsection{Linear Scaling Filter (DsFIs)}

Substitui o pixel central da sliding window usando a seguinte fórmula[27]:

Onde:

$$
f_{i, j}=\frac{g_{\max }+g_{\min }}{2}
$$

$g_{\min }$ é o pixel de menor valor da sliding window

$g_{\text {max }}$ é o pixel de maior valor da sliding window

\subsubsection{Linear Scaling and Sorting Filter (DsFlecasort)}

Atribui o valor médio dos $k$ pixels da vizinhança definida pela sliding window, incluindo o pixel central, ao valor do próprio pixel central, como visto em [28].

\subsubsection{Maximum Homogeneity Over Pixel Neighborhood Filtering (DsFhomog)}

Este filtro[29] estima a área mais homogênea da vizinhança do pixel central de uma sliding window, substituindo o pixel central usando a seguinte formula:

$$
f_{i, j}=\frac{c_{i, j} g_{i, j}}{\sum_{i, j} c_{i, j}}
$$

Onde:

$$
c_{i, j}=\left\{\begin{array}{c}
1 \text { se }\left(1-2 \sigma_{n}\right) \bar{g} \leq g_{i, j} \leq\left(1+2 \sigma_{n}\right) \bar{g} \\
0 \text { caso contrário }
\end{array}\right.
$$

$\bar{g}_{p}=$ média do ruído na janela selecionada

$\sigma_{n}^{2}=$ variância do ruído em toda a imagem, como visto em 3.1.1

\subsubsection{Geometric Filtering (DsFgf4d)}

Partindo do princípio que o ruído speckle aparece como paredes (arestas claras) e vales (arestas escuras) estreitos, usualmente filtros geométricos tentam justamente derrubar as paredes e preencher os vales, borrando assim arestas fracas que precisam ser preservadas.

Já o filtro DsFgf4d incrementa ou decrementa a intensidade do pixel central da sliding window tal que este se torne mais representativo de seu entorno[30].

Isso é feito usando o seguinte procedimento:

$$
\begin{aligned}
& \text { se } a \geq b+2, \text { então } b=b+1 ; \\
& \text { se } a>b \text { e } b \leq c, \text { então } b=b+1 ; \\
& \text { se } c>b \text { e } b \leq a \text {, então } b=b+1 ; \\
& \text { se } c \geq b+2, \text { então } b=b+1 ; \\
& \text { se } a \leq b-2, \text { então } b=b-1 ; \\
& \text { se } a>b \text { e } b \geq c, \text { então } b=b-1 ; \\
& \text { se } c>b \text { e } b \geq a \text {, então } b=b-1 ; \\
& \text { se } c \leq b-2, \text { então } b=b-1 .
\end{aligned}
$$


Onde b é o pixel central e a e c são os pixels vizinhos na direção Norte-Sul, Leste-Oeste, Noroeste para Sudeste e Nordeste para Sudoeste, sendo as direções aplicadas nesta ordem.

\subsubsection{Homomorphic Filtering (DsFhomo)}

Computa a Transformada Rápida de Fourier (FFT) da imagem comprimida logaritimicamente, aplicando uma função H e então a inversa da FFT para retornar ao domínio original. Aqui a função $\mathrm{H}$ usada foi um filtro de Butterworth de highboost e ao final do processamento do DsFhomo[16], ele aumenta a nitidez das features e nivela as variações de speckle na imagem.

$$
\begin{aligned}
& H_{u, v}=\gamma_{L}+\frac{\gamma_{H}}{1+\frac{D_{0}}{D_{u, v}}} \\
& D_{u, v}=\sqrt{(u-N / 2)^{2}+(v-N / 2)^{2}},
\end{aligned}
$$

Onde D0 $=1.8$ é o corte da frequência do filtro, $\gamma_{L}=0.4$ e $\gamma_{H}=0.6$ são os ganhos para baixa e alta frequência, u e $v$ são as coordenadas espaciais da frequência transformada e $\mathrm{N}$ a dimensão da imagem.

\subsubsection{Denoising Sign Dependent Noise (DSDN)}

Foi. [31] desenvolveu esse filtro baseado na regra de interseção de intervalos de confiança (ICI) para estimativas adaptáveis a pontos generalizado para processamento de imagens $2 \mathrm{D}$, onde janelas de tamanhos variáveis são usadas, em conjunto com uma estimativa anisotrópica direcional. Como resultado o estimador é anisotrópico, podendo ter formatos variáveis e não usuais, como visto na Figura 3.3 .

\subsubsection{Shape-Adaptive Discrete Cosine Transform (SADCTD)}
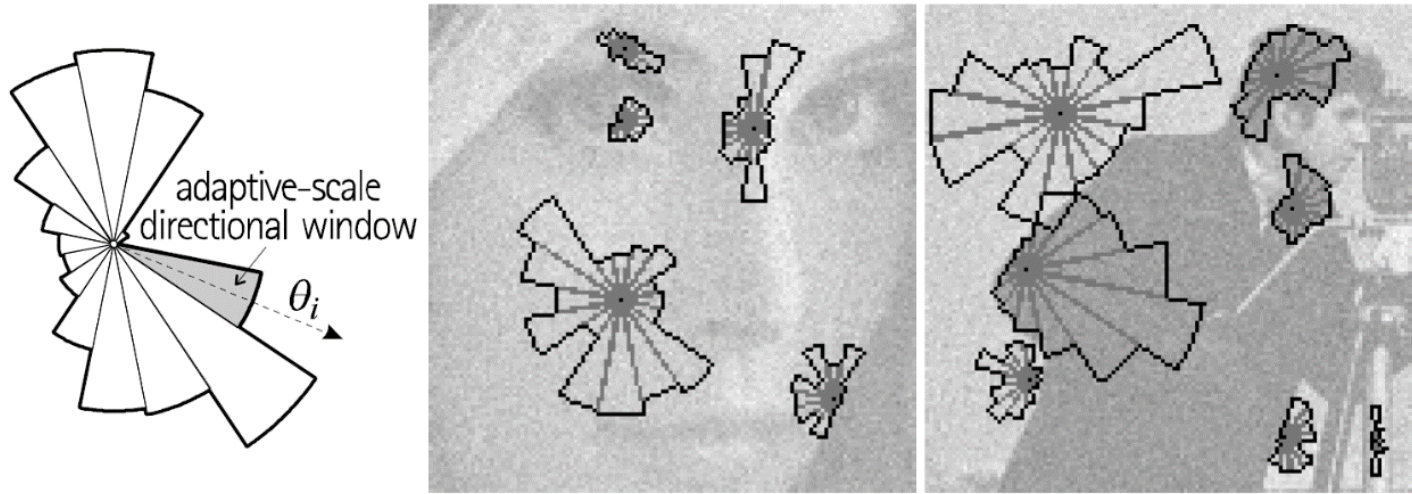

Figura 3.3: Aproximações anisotrópicas locais, resultado da aplicação do algoritmo. Da esquerda para a direita: o estimador anisotrópico adaptativo; exemplos da aplicação do estimador. [31]

Também desenvolvido por Foi, A. et. al[32][33] este filtro usa suportes de formatos arbitrários que são adaptativos em relação a imagem, salientando então seus detalhes e suavizando eficientemente regiões homogêneas, explorando para isso transformadas discretas de cosseno de baixa complexidade.

Aqui este conceito é usado em conjunto com uma Aproximação Local Polinomial (LPA) e a técnica de Interseção de Intervalos de Confiança (ICI), que 
define o formato do suporte da transformada de uma maneira adaptativa ao ponto, sendo assim uma extensão do filtro DSDN.

\subsection{Filtros de suavização de ruído Speckle por Difusão}

São filtros que suavizam o ruído de uma imagem ao modificá-la pela solução de uma equação parcial diferencial.

\subsubsection{Anisotropic Diffusion Filtering (DsFad)}

Perona e Malik [19] substituíram a equação clássica de difusão anisotrópica pela aplicação do coeficiente de difusão, com sua formulação geral apresentada a seguir:

$d(|\nabla g|)=\frac{\mathrm{d} g_{i, j, t}}{\mathrm{~d} t}=\operatorname{div}\left[d_{i, j, t} \nabla g_{i, j, t}\right]=\left[\frac{\mathrm{d} d_{i, j, t}}{\mathrm{~d} i} \frac{\mathrm{d} g_{i, j, t}}{\mathrm{~d} i}\right]+\left[\frac{\mathrm{d} d_{i, j, t}}{\mathrm{~d} j} \frac{\mathrm{d} g_{i, j, t}}{\mathrm{~d} j}\right]$

E a imagem final sendo calculada por pela fórmula geral:

$f_{i, j}=g_{i, j}+\frac{1}{4} \frac{\mathrm{d} g_{i, j}}{\mathrm{~d} t}=g_{i, j}+\frac{1}{4} d(|\nabla g|)$

Entretanto três especializações diferentes da formulação geral foram calculadas neste filtro, sendo substituídas no cálculo da imagem final e podendo assim serem considerados como três filtros distintos, mas que seguem um mesmo padrão, mudando apenas como uma variável desta fórmula geral é calculada.

Onde:

$\operatorname{div}=$ operador de divergência $=\nabla \cdot F$

$|\nabla g|=$ Magnitude do gradiente

$K=$ Parâmetro manual do filtro

$d(|\nabla g|)=$ Funciona como uma função de parada em fronteira, escolhida de forma que $d \rightarrow 0$ quando $|\nabla g| \rightarrow \infty$

1. Perona Malik diffusion equation No 1

$$
d(|\nabla g|)=e^{-\left(\frac{\left|\nabla g_{i, j}\right|}{K}\right)^{2}}
$$

2. Perona Malik diffusion equation No 2

$$
d(|\nabla g|)=\frac{1}{1+\left(\frac{\left|\nabla g_{i, j}\right|}{K}\right)^{2}}
$$

3. Speckle Reducing Anisotropic Diffusion No 3

$$
d(|\nabla g|)=\sqrt{\frac{\left|\left(\frac{\left|\nabla g_{i, j}\right|-\left|\nabla g_{i, j}\right|^{2}}{2}\right) * K\right|}{\max \left(0.01, g+\frac{\nabla g_{i, j}}{4}\right)}}
$$

\subsubsection{Speckle-Reducing Anisotropic Diffusion Filtering (DsFsrad)}

O coeficiente de variação instantânea csrad combina um operador de magnitude de gradiente e um operador laplaciano normalizado para formar um 
detector de arestas em imagens com ruído speckle, onde uma magnitude de gradiente alta combinada com um laplaciano baixo indica uma aresta[34].

$$
\begin{array}{r}
f_{i, j}=g_{i, j}+\frac{1}{\eta_{s}} \operatorname{div}\left(C_{\text {srad }}(|\nabla g|) \nabla g_{i, j}\right) . \\
c_{\text {srad }}^{2}(|\nabla g|) \frac{\frac{1}{2}\left|\nabla g_{i, j}\right|^{2} \frac{1}{16}\left(\nabla^{2} g_{i, j}\right)^{2}}{\left(g_{i, j}+\frac{1}{4} \nabla^{2} g_{i, j}\right)^{2}}
\end{array}
$$

div = operador de divergência, como visto em 3.3.1

$\nabla g=$ Gradiente da imagem ruidosa

$|\nabla g|=$ Magnitude do gradiente

$\nabla^{2} g_{i, j}=$ Operador Laplaciano

$\eta_{s}=$ a vizinhança espacial do filtro e usado neste trabalho com valor igual a 4

\subsubsection{Nonlinear Coherent Anisotropic Diffusion Filtering (DsFIndif)}

Remove o ruído speckle ao modificar a imagem com a solução de uma equação diferencial parcial, enquanto a suavização depende das fronteiras das arestas da imagem e suas direções.

Filtros de anisotropic diffusion[35] são técnicas não lineares para realce de contraste e redução de ruído, suavizando áreas homogêneas enquanto mantém as fronteiras da imagem, utilizando a seguinte fórmula para atingir este objetivo:

$$
\begin{aligned}
& \frac{\mathrm{d} g_{i, j, t}}{\mathrm{~d} t}=\operatorname{div}[D \nabla g] \\
& D=\left(\omega_{1} \omega_{2}\right)\left(\begin{array}{cc}
\lambda_{1} & 0 \\
0 & \lambda_{2}
\end{array}\right)\left(\begin{array}{c}
\omega_{1}^{T} \\
\omega_{2}^{T}
\end{array}\right) \\
& \begin{array}{l}
\lambda_{1}=\left\{\begin{array}{c}
\alpha\left(1-\frac{\left(\mu_{1}-\mu_{2}\right)^{2}}{s^{2}}\right) \text { se }\left(\lambda_{1} \lambda_{2}\right)^{2} \leq s^{2} \\
0 \text { caso contrário }
\end{array}\right. \\
\lambda_{2}=\alpha
\end{array} \\
& \text { Onde: }
\end{aligned}
$$

div $=$ operador de divergência, como visto em 3.3.1

Onde $\omega_{1} e \omega_{2}$ são autovetores, $\lambda_{1} e \lambda_{2}$ são autovalores, e indicam a direção das variações máximas e mínimas e a intensidade dessas variações, respectivamente. O fluxo em cada ponto é afetado pela coerência local, o que é medido por $\mu_{1}-\mu_{2}$, que são autovalores da Decomposição de Valores Singular (SVD) de g.

\subsubsection{Bilateral}

É um filtro de difusão que preserva arestas, resultado do uso de Range e Spacial Kernels, onde aqui ambos são Gaussianos[36][37].

$$
f(x)=\frac{1}{\eta} \int_{\Omega} g_{\delta s}(x-y) g_{\sigma r}(f(x-y)-f(x)) f(x-y) d y
$$

Onde 


$$
\eta=\int_{\Omega} g_{\delta s}(x-y) g_{\sigma r}(f(x-y)-f(x)) d y
$$

$g_{\sigma r}(\mathrm{~s})$ é o kernel de range, dado pela distribuição Gaussiana unidimensional com variância $\sigma_{r}^{2}$. Controlado pela distribuição local de intensidade, ele faz com que descontinuidades pontuais, que tipicamente ocorrem na vizinhança de arestas, sejam usadas para inibir a difusão espacial. Por outro lado, ele se torna inoperante em regiões com variações suaves em intensidades.

$g_{\delta s}(x)$ é o kernel spacial, dado pela distribuição Gaussiana centrada no plano com variância $\sigma_{s}^{2}$, tendo um comportamento contrário ao do kernel de range.

$\Omega$ é o suporte de $g_{\delta s}(x)$ em que a média ocorre.

Juntos, os kernels de range e spacial suavizam regiões mais homogêneas ao mesmo tempo que preservam fronteiras.

O Bilateral pode ainda ser otimizado pela aproximação do kernel de range Gaussiano por uma base de Fourier, onde os coeficientes da base são obtidos pela solução de uma série de problemas de mínimos quadrados, garantindo assim, segundo os autores[38], acurácia em nível de subpixel.

\subsubsection{Trilateral}

O Trilateral[39] combina dois filtros bilaterais modificados com um esquema de empilhamento de imagens para uma rápida pesquisa de regiões com valores de gradientes suavizados semelhantes. Para tal ele traz três principais contribuições:

a.Tilting

Sua sliding window é inclinada pela suavização bilateral para procurar por regiões de alto gradiente, seguindo as seguintes fórmulas:

$$
\begin{aligned}
& f(x)=g(x)+\frac{1}{k_{\Delta}(x)} \int_{\infty}^{\infty} I_{\Delta}(x, \zeta) B(x, \zeta) d \zeta \\
& I_{\Delta}(x, \zeta)=I_{i n}(x+\zeta)-P(x, \zeta) \\
& k_{\Delta}(x)=\int_{\infty}^{\infty} B(x, \zeta) d \zeta \\
& B(x, \zeta)=c(\zeta) s\left(I_{\Delta}(x, \zeta)\right) f_{\theta}(x, \zeta) \\
& P(x, \zeta)=I_{\text {in }}(x)+G_{\theta} \cdot \zeta \\
& k_{\theta}(x)=\int_{\infty}^{\infty} C(x, \zeta) d \zeta \\
& G_{\theta}(x)=\frac{1}{k_{\theta}(x)} \int_{\infty}^{\infty} \nabla I_{\text {in }}(x+\zeta) C(x, \zeta) d \zeta \\
& C(x, \zeta)=c(\zeta) s\left(\left\|\nabla I_{\text {in }}(x+\zeta)-\nabla I_{\text {in }}(x)\right\|\right)
\end{aligned}
$$

Onde:

$c(\cdot)=$ Kernel de domínio da função escolhida, que apesar de poder ser qualquer função, aqui foi escolhida como uma Gaussiana de variância $\sigma_{c}$. Esta função determina um peso escalar dependente da posição para a contribuição de cada ponto da imagem final.

$\zeta=$ Vetor de offset no domínio em volta de $\mathrm{x}$.

$\mathrm{x}=$ Pixel corrente.

$s(\cdot)=$ Filtro de alcance (range) que atribui pesos aos valores dos vizinhos de acordo com sua diferença de intensidade com $\mathrm{g}(\mathrm{x})$. Assim como $\mathrm{c}(\cdot), \mathrm{s}(\cdot)$ também pode ser qualquer função e foi escolhida como uma Gaussiana de variância $\sigma_{\mathrm{s}}$. 
$k_{\theta}(\mathrm{x})$ e $\mathrm{k}_{\Delta}(\mathrm{x})=$ Usada para normalização dos pesos.

$I_{\Delta}(\mathrm{x}, \zeta)=$ Sinal de detalhe local.

$G_{\theta}(\mathrm{x})=$ Vetor de inclinação, responsável por atribuir a mesma média para vizinhos com gradientes semelhantes, enquanto ignora gradientes fortemente diferentes (outliers).

b.Adaptative Region-Growing $f_{\theta}$ :

Os vizinhos locais (ou domínio) automaticamente se adaptam às características locais da imagem para suavizar a maior área possível com valores de gradientes suavizados semelhantes, atingindo isso com:

$$
f_{\theta}(x, \zeta)=\left\{\begin{array}{c}
1 \text { se }\left\|G_{\theta}(x+\zeta)-G_{\theta}(x)\right\|<R \\
0 \text { caso contrário }
\end{array}\right.
$$

Onde:

$f_{\theta}(\mathrm{x}, \zeta)=$ Função binária, $[0,1]$, que limita a vizinhança suavizada para áreas conexas de $\mathrm{x}$ que partilham de vetores $\left\|\mathrm{G}_{\theta}\right\|$ similares.

$R=$ Threshold escalar usado para determinar o que são valores similares.

c.Um único parâmetro:

Todos os 7 parâmetros internos do trilateral $\left(\sigma c, \sigma_{c} \theta, \sigma_{r}, \sigma_{r} \theta, f \theta, R, \beta\right)$ são derivados de um único parâmetro manual.

\subsection{Outros}

Esta categoria possui filtros que utilizam conceitos presentes nas outras categorias, ora utilizando estatísticas da imagem como forma de melhor estimar o ruído, como observado nos Filtros Lineares, ora utilizando todo o conteúdo de uma sliding window para suprimir o ruído, como visto nos Filtros Não Lineares, ora apresentando conceitos novos como médias não locais (Non-Local Means) e, portanto, não sei encaixam nas categorias apresentadas anteriormente.

\subsubsection{Field of Experts with a Nakagami Distribution (FoE_Naka)}

Utiliza o conceito de Fields of Experts (FoE) para modelar a probabilidade a priori de uma imagem, funcionando como um filtro baseado em um modelo de Markov Random Fields (MRF) de ordem superior, caracterizando explicitamente propriedades estatísticas de uma imagem[40].

O modelo de filtragem speckle é definido pelo seguinte problema de minimização de energia:

$\hat{u}=\arg \min _{u} E(f, g)=E_{F o E}(f)+D(f, g)$

Onde o modelo FoE é definido como um conjunto de filtros lineares e segue a seguinte formula:

$$
\begin{aligned}
& E_{F O E}(f)=\sum_{i=1}^{N_{f}} \theta_{i} \rho\left(k_{i} * f\right) \\
& \rho\left(k_{i} * f\right)=\sum_{p=1}^{N} \rho\left(\left(k_{i} * f\right)_{p}\right)
\end{aligned}
$$




$$
\rho(x)=\log \left(1+x^{2}\right)
$$

Onde:

$\mathrm{N}=$ número de pixels na imagem $\mathrm{u}$.

$\mathrm{N}_{\mathrm{f}}=$ quantidade de filtros lineares.

$\mathrm{k}_{\mathrm{i}}=$ conjunto de filtros aprendidos com seus correspondentes pesos $\theta_{\mathrm{i}}>0$.

$\mathrm{k}_{\mathrm{i}}^{*} \mathrm{u}=$ convolução do filtro $\mathrm{k}_{\mathrm{i}}$ com a imagem bidimensional $\mathrm{u}$.

$\rho(x)$ = função não-convexa potencial Lorentziano, derivado de uma distribuição tstudent.

A segunda parte da minimização, $D(f, g)$, é o termo de fidelidade, derivado do ruído multiplicativo e aqui seguindo a distribuição de Nakagami.

Os autores notaram que usando um termo de fidelidade com boa acurácia em relação ao modelo de ruído multiplicativo geraram resultados melhores que um termo "impróprio", entretanto ambos os modelos possuíam forças e fraquezas complementares, resultando assim na seguinte equação:

$$
D(f, g)=\frac{\lambda_{1}}{2}\left(2 w+g^{2} e^{-2 w}\right)+\frac{\lambda_{2}}{2}\left(e^{2 w}-2 g^{2} w\right)
$$

Esta equação é resolvida usando um algoritmo de otimização não-convexo altamente eficiente chamado iPiano[41], desenvolvido para problemas de otimização estruturados, não-suavizados e não-convexos.

Onde:

$\mathrm{f}=\mathrm{e}^{\mathrm{w}}$. É uma imagem sem ruído desconhecida.

$\mathrm{W}=\log (\mathrm{f})$

$\lambda_{1}$ e $\lambda_{2}$ parâmetros ajustados manualmente.

\subsubsection{Wavelet Filtering (DsFwaveltc)}

Wavelets são conhecidos pela sua capacidade de identificar singularidades associadas com pequenas variações do sinal observado.

O DsFwaveltc estima a variância do ruído speckle, computa a transformada de wavelet discreta (DWT) com o Symlet Wavelet para duas escalas, computa um threshold $\mathrm{T}$ para cada subbanda e o aplica nos coeficientes de wavelet para cada banda para finalmente calcular a inversa do DWT e assim reconstruir a imagem sem ruído[16].

$$
T=\left\{\begin{array}{c}
\left(T_{\max }-\alpha(j-1)\right) \sigma_{n} \text { se }\left(T_{\max }-\alpha(j-1)\right) \geq T_{\min }, \\
T_{\min } \sigma_{n} \text { caso contrário }
\end{array}\right.
$$

Onde:

$\alpha$ é um fator decrescente entre dois níveis consecutivos

$T_{\text {max }}$ é $o$ maior fator de variância $\left(\sigma_{n}\right)$

$T_{\text {min }}$ é $o$ menor fator de variância $\left(\sigma_{n}\right)$

$\sigma_{n}=$ desvio padrão do ruído

\subsubsection{L0 Smoothing}

Possui um esquema de contagem de gradientes esparsos, com sua principal contribuição sendo uma nova estratégia para confinar o número discreto de mudanças de intensidade ao longo de pixels vizinhos, o que corresponde 
matematicamente à norma L0 para encontrar informações esparsas. O efeito qualitativo deste método é delimitar bordas salientes, tornando-as mais fáceis de serem detectadas e mais visualmente distintas.

O uso da minimização do gradiente L0 elimina uma quantidade razoável de estruturas de baixa amplitude ao mesmo tempo que realça arestas importantes de uma imagem[42].

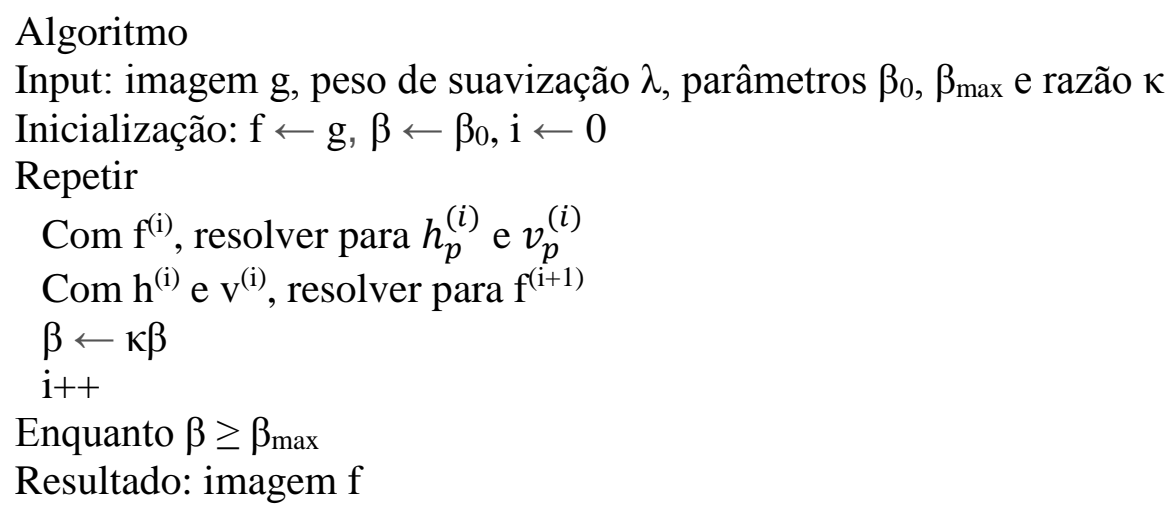

$$
\begin{aligned}
& \left(h_{p}, v_{p}\right)= \begin{cases}(0,0) & \text { se }\left(\partial_{x} S_{p}\right)^{2}+\left(\partial_{y} f_{p}\right)^{2} \leq \lambda / \beta \\
\left(\partial_{x} f_{p}, \partial_{y} f\right) & \text { caso contrário }\end{cases} \\
& f=\mathcal{F}^{-1}\left(\frac{\mathcal{F}(g)+\beta\left(\mathcal{F}\left(\partial_{x}\right)^{*} \mathcal{F}(h)+\mathcal{F}\left(\partial_{y}\right)^{*} \mathcal{F}(v)\right)}{\mathcal{F}(1)+\beta\left(\mathcal{F}\left(\partial_{x}\right)^{*} \mathcal{F}\left(\partial_{x}\right)+\mathcal{F}\left(\partial_{y}\right)^{*} \mathcal{F}\left(\partial_{y}\right)\right)}\right)
\end{aligned}
$$

Onde:

$\mathcal{F}$ é o operador de Transformada Rápida de Fourier.

$\mathcal{F}^{-1}$ é o operador de Transformada Inversa Rápida de Fourier.

$p$ é uma denotação para pixel.

$\mathrm{h}_{\mathrm{p}}$ e $\mathrm{v}_{\mathrm{p}}$ são variáveis auxiliares que correspondem a $\partial_{x} f_{p}$ e $\partial_{y} f_{p}$, respectivamente.

$\beta$ é um parâmetro de controle de similaridade entre as variáveis $(h, v)$ e seus respectivos gradientes.

$\lambda$ é um parâmetro de suavização.

$\kappa$ determina a velocidade de convergência do algoritmo.

\subsubsection{Bilateral and LO Smoothing}

Como o L0 Smoothing não usa filtros espaciais ou médias, ele pode ser considerado como complementar a abordagens locais anteriores.

A aplicação do filtro bilateral diminui a amplitude de estruturas ruidosas mais que a amplitude de arestas longas e coerentes. Quando seguida da aplicação do L0 Smoothing, este irá ressaltar arestas proeminentes globalmente, ou seja, a aplicação do Bilateral seguida do L0 acaba por suavizar o ruído ao mesmo tempo que realça arestas importantes[42].

\subsubsection{Probabilistic Patch Based (PPB_Nakagami)}

Baseado no método de médias não locais, os autores[43] alteraram a maneira como o cálculo de similaridade de pixels era feito, usando um critério de 
similaridade estatística, dependente do modelo de distribuição de ruído de Nakagami-Rayleigh, ao invés da distância euclidiana entre patches.

O método de suavização de ruído é definido como um WMLE, conhecido por reduzir o erro médio quadrático ao reduzir a variância do estimador ao custo da introdução de um viés.

A generalização dos pesos baseados na distância euclidiana encontrados nos algoritmos de médias não locais é feita no filtro PPB de forma probabilística, que é ainda refinado iterativamente ao incluir patches dos parâmetros estimados da imagem. O processo final é descrito da seguinte maneira, que pode também ser visto na Figura 3.4:

1. O Estimador de Pesos do PPB (PPBWE) calcula os pesos $w(s, t)$ ao usar a imagem ruidosa $v$ para o termo de verossimilhança e para o termo a priori.

2. O WMLE computa os novos parâmetros ao usar o PPBWE $w(s, t)$ e a imagem ruidosa $v$.

3. Os passos 1 e 2 são repetidos até que a divergência simétrica de KullbackLeibler convirja a zero.

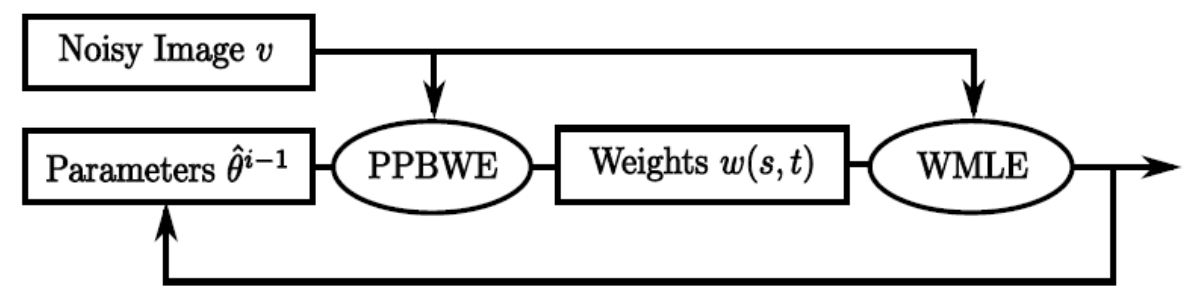

Figura 3.4 - Esquema do filtro PPB Iterativo [43].

Finalmente, os pesos são definidos por

$$
w(s, t)=\left\{-\sum_{k}\left[\frac{2 L-1}{h} \log \left(\frac{A_{s, k}}{A_{t, k}}+\frac{A_{t, k}}{A_{s, k}}\right)+\frac{1}{T} \frac{\left|\hat{R}_{s, k}^{i-1}+\hat{R}_{t, k}^{i-1}\right|^{2}}{\hat{R}_{s, k}^{i-1} \hat{R}_{t, k}^{i-1}}\right]\right\}
$$

Onde o equilíbrio entre supressão de ruído e fidelidade na estimativa é atingido pelo parâmetro manual $\mathrm{T}$ e por $\mathrm{h}$, um alpha-quantil da distribuição que possui um parâmetro alpha também determinado manualmente;

$\mathrm{L}=$ Number of Looks e igual a 1 nesse trabalho.

$\mathrm{A}=$ Amplitude dos pixels.

$R^{*}=$ Refletância da imagem.

$\hat{R}=$ Estimativa da refletância sem ruído.

$A^{*}=\sqrt[2]{R^{*}}$

$A_{t, k}$ e $A_{s, k}$ são os k-ésimos pixels de amplitudes, onde seus valores anteriores são $\hat{R}_{s, k}^{i-1}$ e $\hat{R}_{t, k}^{i-1}$ para patches t e s.

\subsubsection{Synthetic Aperture Radar Block Matching 3D (SARBM3D)}

Tido como o estado da arte em suavização de ruído speckle, este filtro traz modificações ao BM3D[21], uma vez que este foi desenvolvido em cima da hipótese de tratamento de ruído aditivo branco gaussiano (Additive White Gaussian Noise, AWGN) e o ruído speckle é tido principalmente como multiplicativo. A estrutura do algoritmo usado pelo SARBM3D é análoga à do BM3D, adaptando-o às características e estatísticas específicas do ruído speckle. 
O algoritmo final funciona em duas etapas, a primeira é usada para criar uma imagem relativamente limpa para que seja possível estimar estatísticas relevantes, enquanto a segunda etapa realiza a suavização em si. Ambos funcionam em grupos de blocos de diferentes partes da imagem, que são selecionados de acordo com uma medida de similaridade[44].

O termo agrupamento, usado em ambas as etapas do algoritmo, se refere ao conceito de colecionar fragmentos (blocos ou patches) 2D da imagem de entrada em uma estrutura 3D, chamada de grupo, ao empilhar fragmentos semelhantes, como visto na Figura 3.5. Este passo é importante uma vez que ele permite o uso de filtros com dimensões maiores para cada grupo, que exploram a potencial similaridade (correlação, afinidade, etc.) entre fragmentos agrupados para estimar o sinal real em cada um deles, sendo essa abordagem chamada de filtragem colaborativa.

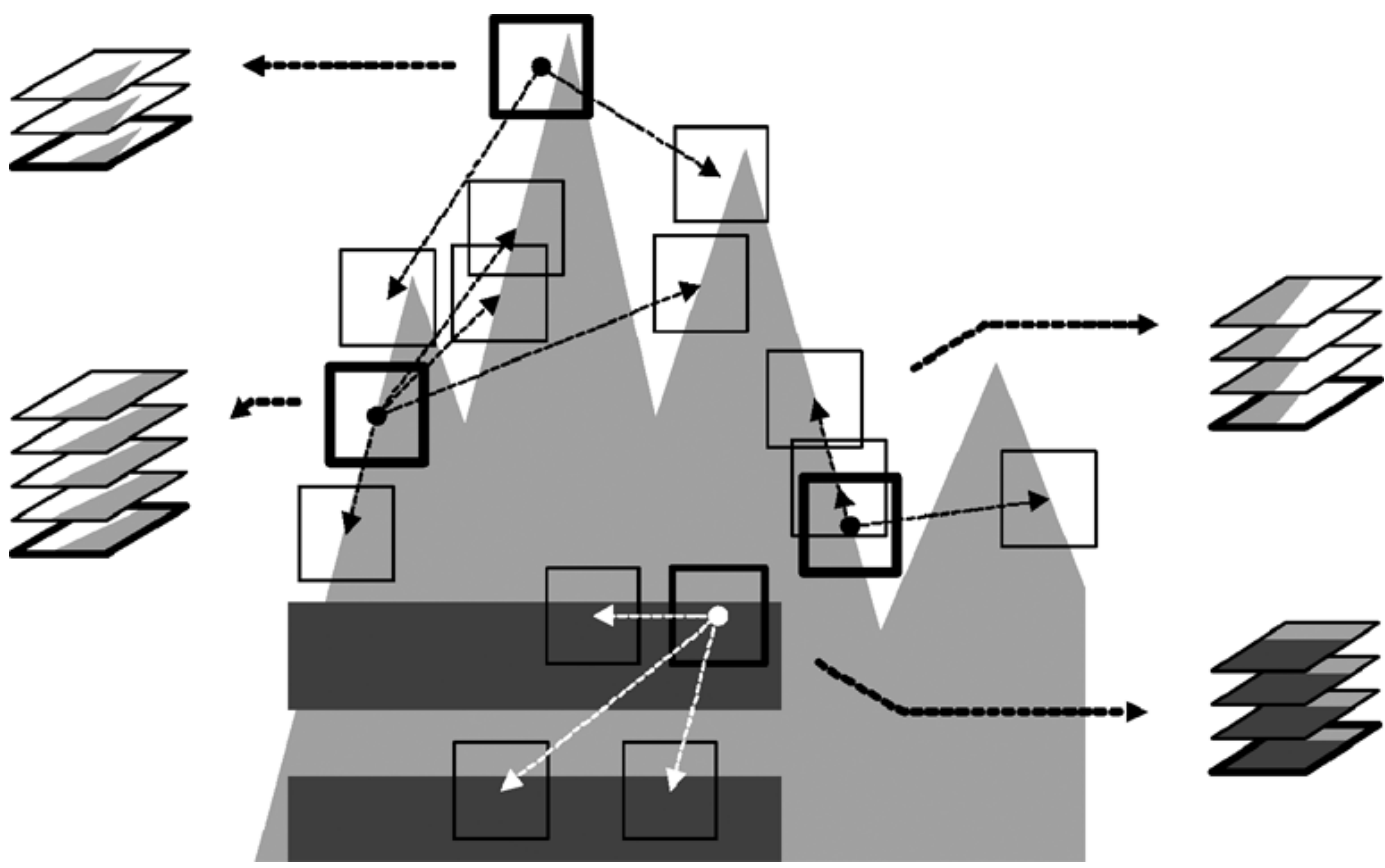

Figura 3.5: Exemplo do passo de agrupamento em uma imagem artificial, em que cada bloco de referência possui outros blocos semelhantes [21].

Cada etapa é dividida em três estágios:

1) Agrupamento: Para cada bloco de referência, os blocos mais similares são localizados na imagem de acordo com uma função de flutuação na imagem, que representa a semelhança que duas observações correspondem à mesma radiância sem ruído na cena.

2) Filtragem colaborativa: Cada grupo $3 \mathrm{D}$ passa por uma filtragem de Local Linear Minimum Mean Squared Error (LLMMSE) com uso de uma Undecimated Discrete Wiener Transform (UDWT)

3) Agregação: Todos os blocos filtrados retornam para sua localização original e contribuem com pesos para a estimativa base da imagem. Esses pesos são feitos para dependerem da confiabilidade presumida da estimativa dos grupos associados, relacionados em troca ao poder médio do ruído do grupo após o encurtamento provido pelo LLMMSE. 
A segunda etapa possui os mesmos três estágios, com as seguintes diferenças:

1) Agrupamento: A medida de similaridade deve levar em consideração a nova informação obtida na primeira etapa, que é uma estimativa grosseira do sinal sem ruído.

2) Filtragem colaborativa: Uma vez com uma estimativa do coeficiente do sinal sem ruído, a complexidade desta etapa pode ser diminuída, possibilitando assim o uso da filtragem de Wiener empírica.

3) Agregação: Acontece da mesma maneira que na etapa anterior. 


\section{Métricas}

Para impedir que a seleção de um filtro ficasse exclusivamente a cargo de avaliações visuais, foram feitos diversos testes visando uma melhor avaliação dessa escolha, bem como sua qualidade, consistência e melhor definição dos parâmetros usados para cada filtro. Estes testes baseiam-se em métricas, que atribuem uma pontuação a uma imagem para que estas possam ser facilmente comparadas entre si.

Um total de 21 métricas foram escolhidas para avaliar o desempenho de cada filtro e nas fórmulas apresentadas a seguir, $g$ corresponde à imagem ruidosa de entrada, enquanto $f$ representa a imagem filtrada e após cada fórmula encontramse seus limites inferior e superior teóricos, quando possível.

\subsection{Tipos de Métricas}

As métricas foram divididas em três grupos[45] para melhor comparação das métricas entre si, onde os grupos diferem entre si pelo tipo de comparação feita entre imagens:

1. Referência Completa (Full Reference)

2. Referência Reduzida (Reduced Reference)

3. Sem Referência (No Reference)

\subsubsection{Referência Completa}

As métricas de referência completa são aquelas que comparam duas imagens entre si, sendo uma delas geralmente proveniente de algum processo de filtragem e a outra uma imagem ideal, um ground-truth, onde esta seria uma imagem sem ruído algum, que não necessita de nenhum tipo de realce ou suavização. Contudo, obter uma imagem ideal para ser comparada com uma imagem filtrada, no caso de exames de ultrassom, não é trivial.

Não foram encontrados métodos confiáveis de geração de ground-truth, apesar de existirem maneiras de contornar este problema, como o framework Field II[46], capaz de simular qualquer sistema linear de ultrassom com diversas características. Entretanto existe pouca documentação sobre como melhor usar esta ferramenta, além dela ser extremamente custosa computacionalmente, podendo levar dias para completar uma simulação.

Outro problema encontrado ao se usar o Field II é que sua simulação acaba sendo uma aproximação do modelo speckle, sendo assim necessário levantar a discussão da incerteza acerca de se o resultado obtido com o dado simulado é válido também para o caso do dado real.

A primeira maneira seria fazer o caminho inverso, pegando uma imagem de ressonância magnética, por exemplo, e gerar tanto o ground-truth artificial, ajustando intensidade do sinal e espaçamento de amostragem, quanto a imagem ruidosa, aplicando um método de geração de ruído para que esta se assemelhe a uma imagem de ultrassom. Seu problema encontra-se no fato de que o ruído speckle, presente em todo e qualquer exame de ultrassom, não segue um modelo simples, não sendo assim facilmente simulável. Dentre os motivos estão a forte auto correlação espacial com as estruturas na região analisada. 
A segunda maneira é usar a ajuda de um especialista para limpar manualmente ou de forma semiautomática uma imagem de ultrassom, realçando as características necessárias e suavizando áreas de interesse.

Esta abordagem tem a vantagem de contar com a análise de informações do volume em torno da imagem bidimensional sendo trabalhada. Em contrapartida, a fragilidade deste método encontra-se justamente no julgamento do especialista sobre a imagem de ultrassom, podendo ele não ser consistente em todas as limpezas, realizando pouca ou muita suavização e realce.

Visando realizar uma comparação o mais fiel possível com os exames de ultrassom obtidos, optou-se pelo segundo método, contando com a ajuda de um especialista em tratamento manual e semiautomático de exames de ultrassom para reconstrução e impressão 3D.

Como o julgamento do especialista é um fator crucial nesta decisão, o resultado das métricas não será, portanto, definitivo, uma vez que o ground truth gerado não é perfeito, sendo ainda necessário que uma validação visual seja feita para comprovar que os resultados obtidos de fato condizem com as diretivas apresentadas no início do capítulo anterior.

\subsubsection{Average Difference (AD)}

A diferença média entre a imagem original e filtrada é definida por [47]:

$$
\begin{gathered}
A D=\frac{\left|\sum_{i=1}^{M} \sum_{j=1}^{N} g_{i, j}-f_{i, j}\right|}{M N} \\
0 \leq A D \leq 255
\end{gathered}
$$

\subsubsection{Mean Square Error (MSE)}

Mede a diferença de qualidade entre a imagem original e a processada em uma janela de $\mathrm{MxN}$, correspondentes ao tamanho das imagens observadas. Quando somente esta métrica é utilizada para fins comparativos ela não se correlaciona de maneira forte o suficiente com a qualidade percebida e, portanto, deve ser usada em conjunto com outras métricas [48][49].

$$
\begin{gathered}
M S E=\frac{1}{M N} \sum_{i=1}^{M} \sum_{j=1}^{N}\left(g_{i, j}-f_{i, j}\right)^{2} \\
0 \leq M S E \leq 255^{2}
\end{gathered}
$$

\subsubsection{Momentos de Minkowski M3 e M4}

O somatório do erro na forma de uma métrica de Minkowski, que é a norma da dissimilaridade entre a imagem original e filtrada, aqui usado com $\beta$ igual a $3 \mathrm{e}$ 4 para métricas M3 e M4, respectivamente [50]. Repare que para $\beta=1$ a fórmula corresponde à métrica $\mathrm{AD}$ e para $\beta=2$ ao RMSE.

$$
\begin{gathered}
E r r=\sqrt[\beta]{\frac{1}{M N} \sum_{i=1}^{M} \sum_{j=1}^{N}\left(\left|g_{i, j}-f_{i, j}\right|\right)^{\beta}} \\
0 \leq E r r \leq 255
\end{gathered}
$$




\subsubsection{Signal-to-Noise Error (SNR)}

É uma medida da força do sinal relativo ao ruído[51].

$$
\begin{gathered}
S N R=10 \log _{10} \frac{\sum_{i=1}^{M} \sum_{j=1}^{N}\left(g_{i, j}^{2}+f_{i, j}^{2}\right)}{\sum_{i=1}^{M} \sum_{j=1}^{N}\left(g_{i, j}-f_{i, j}\right)^{2}} \\
0<S N R<10^{6}
\end{gathered}
$$

\subsubsection{Universal Quality Index (UQI)}

Modela qualquer distorção como uma combinação de três fatores diferentes: perda de correlação, distorção em luminância e distorção de contraste[52].

$$
\begin{gathered}
Q=\frac{\sigma_{g f}}{\sigma_{f} \sigma_{g}} \cdot \frac{2 \bar{f} \bar{g}}{(\bar{f})^{2}+(\bar{g})^{2}} \cdot \frac{2 \sigma_{f} \sigma_{g}}{\sigma_{f}^{2}+\sigma_{g}^{2}} \\
-1<U Q I<1
\end{gathered}
$$

Onde $\bar{f}$ e $\bar{g}$ e representam a mediana da imagem original e filtrada com seus desvios padrões $\sigma_{f}$ e $\sigma_{g}$ da janela de analise, enquanto $\sigma_{g f}$ representa a covariância entre a janela, de tamanho $8 \times 8$, original e a filtrada.

\subsubsection{Structural Similarity Index Map (SSIM)}

O SSIM é um melhoramento sobre a métrica anterior. Dada a formulação matemática formal da UQI, quando ambas as médias ou ambas as variâncias se aproximam de zero, uma indeterminação pode emergir durante os cálculos. Deste modo, cada termo é acrescentado de uma constante para evitar possíveis divisões por zero e manter a propriedade de resultar em 1 quando as duas imagens fornecidas forem a mesma. Contudo, o valor selecionado para estas constantes pode afetar a ordem dos resultados e, em caso particular, se ambas as constantes forem anuladas, obtém-se UQI. Sua fórmula é dada por [50]:

$$
\begin{gathered}
\operatorname{SSIM}=\frac{\left(2 \bar{f} \bar{g}+c_{1}\right)\left(2 \sigma_{g f}+c_{2}\right)}{\left((\bar{g})^{2}+(\bar{f})^{2}+c_{1}\right)\left(\sigma_{g}^{2}+\sigma_{f}^{2}+c_{2}\right)} \\
-1<\text { SSIM }<1
\end{gathered}
$$

Onde $c_{1}=0.01 d_{r}$ e $c_{2}=0.03 d_{r}$, com $d_{r}=255$ representando o intervalo dinâmico de imagens de ultrassom e $\bar{f}$ e $\bar{g}$ as medianas da imagem original e filtrada.

\subsubsection{Structural Content (SC)}

Uma métrica baseada em correlação que mede a similaridade entre duas imagens[53], definida por:

$$
\begin{gathered}
S C=\frac{\sum_{i=1}^{M} \sum_{j=1}^{N}\left(f_{i, j}\right)^{2}}{\sum_{i=1}^{M} \sum_{j=1}^{N}\left(g_{i, j}\right)^{2}} \\
0<S C<M N \cdot 255^{2}
\end{gathered}
$$




\subsubsection{Normalized Cross-Correlation (NK)}

Assim como na métrica SC, a NK também é baseada em correlação e por si só, é um método aplicado em processamento de sinais para buscar sequências curtas de um padrão em sequências mais longas, podendo assim ser aplicada para detectar translações entre a imagem original e estimada. Esta métrica segue a seguinte equação [53]:

$$
\begin{gathered}
N K=\frac{\sum_{i=1}^{M} \sum_{j=1}^{N} f_{i, j} * g_{i, j}}{\sum_{i=1}^{M} \sum_{j=1}^{N}\left(f_{i, j}\right)^{2}} \\
0<N K<255
\end{gathered}
$$

\subsubsection{Maximum Difference (MD)}

MD (Maximum Difference) é avaliado em função do módulo das diferenças, para evitar o comprometimento dos resultados pelo clareamento ou escurecimento do resultado do filtro de imagem. Seu significado é complementar ao MSE, visto que uma região da imagem reconstruída com desvio elevado em relação à imagem original fará o erro quadrático médio se elevar, enquanto um MSE elevado com MD baixo implica necessariamente em desvios acumulados em ampla região da imagem, neste caso, AD deve ser consultada para determinar se os desvios foram todos no mesmo sentido (clareamento ou escurecimento geral da imagem).

A maior diferença entre duas imagens é definida por [47]:

$$
\begin{gathered}
M D=\max (\max (|f-g|)) \\
0 \leq M D \leq 255
\end{gathered}
$$

\subsubsection{Laplacian Mean Squared Error (LMSE)}

O LMSE é baseado na importância da medição de arestas e sua definição pode ser vista a seguir[47]:

$$
\begin{aligned}
& L M S E=\frac{\sum_{i=1}^{M} \sum_{j=1}^{N}\left(4 L\left(f_{i, j}\right)-4 L\left(g_{i, j}\right)\right)^{2}}{\sum_{i=1}^{M} \sum_{j=1}^{N}\left(4 L\left(f_{i, j}\right)\right)^{2}} \\
& 0<L M S E<(M N \cdot 2 \cdot 255)^{2} \\
& L(\cdot)=\text { Aplicação do Operador de Laplace }
\end{aligned}
$$

\subsubsection{Normalized Absolute Error (NAE)}

O erro absoluto normalizado é calculado pela razão entre o cumulativo dos valores absolutos dos desvio e o cumulativo dos valores absolutos da imagem original. De certo modo, é similar ao coeficiente de variação, embora utilize erros em módulo no lugar do quadrado das diferenças, sendo definido segundo a seguinte fórmula[47]:

$$
\begin{aligned}
N A E & =\frac{\sum_{i=1}^{M} \sum_{j=1}^{N}\left|f_{i, j}-g_{i, j}\right|}{\sum_{i=1}^{M} \sum_{j=1}^{N}\left|f_{i, j}\right|} \\
0 & <N A E<M N \cdot 255
\end{aligned}
$$




\subsubsection{Coefficient of Correlation(COC)}

Também conhecido como Pearson Correlation Coefficient (PCC), é uma medida de dependência linear entre duas imagens, definida por[54]:

$a=f-\operatorname{mean}(f)$

$$
\operatorname{COC}=\frac{\sum_{i=1}^{M} \sum_{j=1}^{N} a_{i, j} * b_{i, j}}{\sqrt{\left(\sum_{i=1}^{M} \sum_{j=1}^{N} a_{i, j}^{2}\right) *\left(\sum_{i=1}^{M} \sum_{j=1}^{N} b_{i, j}^{2}\right)}}
$$

$b=g-\operatorname{mean}(g)$

\subsubsection{Pratt Figure of Merit (FOM)}

Pratt[55] introduziu um método para avaliar quantitativamente a performance de detectores de arestas, então seu uso depende de uma aplicação a priori de um Canny Edge Detector[56].

$$
\begin{aligned}
F o M(f, g)= & \frac{1}{\max \left(N_{f}, N_{g}\right)} \sum_{j=1}^{N_{f}} \frac{1}{1+\gamma d_{j}^{2}} \\
0 \leq F O M \leq 1 &
\end{aligned}
$$

Onde $\gamma$ representa um multiplicador escalar utilizado como fator de penalidade com valor típico de $1 / 9, N_{f}$ e $N_{g}$ são os números de pixels nas imagens originais e processadas, respectivamente, $d_{j}$ é a distância euclidiana.

\subsubsection{Referência Reduzida}

Métricas de referência reduzida são aquelas que não precisam de uma imagem ground-truth para atribuir uma pontuação a uma imagem. Neste caso as imagens de comparação usadas são a própria imagem ruidosa e a imagem filtrada. Embora consideradas no planejamento do modelo de seleção de filtros, seus resultados se mostraram excessivamente influenciados pela imagem usada para comparação.

Como consequência, este conjunto de métricas favorece filtros de imagem que provocam pouca ou nenhuma alteração quando a imagem de referência fornecida possui ruído intenso similar ao speckle.

\subsubsection{Beta}

Mede a performance da preservação de arestas[57], segundo a fórmula:

$$
\begin{aligned}
\beta & =\frac{\Gamma\left(\Delta f_{g}-\overline{\Delta f}_{f}, \Delta f_{f}-\overline{\Delta f}_{f}\right)}{\sqrt{\Gamma\left(\Delta f_{g}-\overline{\Delta f}_{g}, \Delta f_{g}-\overline{\Delta f}_{g}\right) \cdot \Gamma\left(\Delta f_{f}-\overline{\Delta f}_{f}, \Delta f_{f}-\overline{\Delta f}_{f}\right)}} \\
\Gamma\left(s_{1}, s_{2}\right) & =\sum_{(i, j) \in R O I} s_{1}(i, j) \cdot s_{2}(i, j)
\end{aligned}
$$


Onde $\Delta f_{f}$ e $\Delta f_{g}$ representam a versão passa-alta das imagens original e processadas obtidos pela aplicação do Operador de Laplace de tamanho 3x3, e $\overline{\Delta f}_{f}$ e $\overline{\Delta f}_{g}$ representam a média de intensidade de pixels na região $\Delta f_{f} e \Delta f_{g}$, respectivamente.

\subsubsection{Speckle Supression Index (SSI)}

A razão entre desvio padrão e coeficiente de variância é usado para medir a força do ruído speckle na imagem e, portanto, o SSI pode ser definido por [57]:

$$
\begin{aligned}
S S I= & \frac{\sqrt{\operatorname{VAR}(g)}}{\operatorname{Mean}(g)} \frac{\operatorname{Mean}(f)}{\sqrt{\operatorname{VAR}(f)}} \\
& 0<S S I<255^{2}
\end{aligned}
$$

Em que VAR representa a variância da imagem e Mean sua média.

\subsubsection{Speckle Suppression and Mean Preservation Index (SMPI)}

O SSI não é considerado uma métrica confiável quando os filtros aplicados alteram demais a imagem de entrada. Portanto esta métrica é usada para estimar simultaneamente a supressão do ruído speckle e a preservação da média da imagem filtrada[59], como visto a seguir:

$$
\begin{gathered}
S M P I=Q \times \frac{\sqrt{\operatorname{VAR}(g)}}{\sqrt{\operatorname{VAR}(f)}} \\
0<S M P I<2 \cdot 255^{2}
\end{gathered}
$$

$Q=K+|\operatorname{Mean}(f)-\operatorname{Mean}(g)|$

$K=\frac{\max (\operatorname{Mean}(g))-\min (\operatorname{Mean}(g))}{\operatorname{Mean}(f)}$

VAR = Variância da imagem

\subsubsection{Edge Save Index (ESI)}

Reflete a capacidade de preservação de arestas da técnica de filtragem de ruído empregada. O autor define a métrica ESI tanto para a direção horizontal $h(x)$, quanto para a vertical $v(x)$ [60], e aqui, para simplicidade, a métrica será denotada como a diagonal formada por ambas as direções:

$$
\begin{aligned}
E S I & =\sqrt{\left(\frac{h(g)}{h(f)}\right)^{2}+\left(\frac{v(g)}{v(f)}\right)^{2}} \\
0 & <E S I<255 \cdot M N
\end{aligned}
$$

$$
\begin{aligned}
& h(x)=\sum_{i=1}^{M} \sum_{j=1}^{N-1}|x(i, j+1)-x(i, j)| \\
& v(x)=\sum_{i=1}^{M-1} \sum_{j=1}^{N}|x(i+1, j)-x(i, j)|
\end{aligned}
$$




\subsubsection{Comparison Quality (CQ)}

Esta métrica é formada por dois módulos básicos, o de Detecção de Conteúdo e o de Contribuição, tendo o índice comparativo de qualidade definido por CQ e o índice de qualidade final definido por CQI[45].

\section{Detecção de Conteúdo}

É um módulo qualitativo responsável por identificar o tipo de conteúdo presente na diferença entre as imagens de entrada, se ruído ou estrutura.

\section{Contribuição}

É um módulo quantitativo responsável por identificar qual das imagens de entrada contribui mais para a imagem de diferença, onde a medida de contribuição é determinada pela covariância da luminância normalizada entre imagem de entrada e imagem de diferença.

$$
\begin{aligned}
& \qquad Q I=\frac{1}{M N} * \sum_{i, j=\left(\frac{n}{2}\right):\left(M-\frac{n}{2}\right)} C Q(i, j) \\
& C Q=i s_{-} \text {stru } \cdot c t r i
\end{aligned}
$$$$
\text { Algoritmo 1: Detecção de Conteúdo }
$$$$
D_{p}=P_{1}-P_{2}
$$$$
G=\left[d_{x}\left(D_{p}\right) d_{y}\left(D_{p}\right)\right]
$$$$
U S V^{T}=\operatorname{SVD}(G)
$$$$
C_{\text {ind }}=\frac{s_{1}-s_{2}}{s_{1}+s_{2}}
$$$$
\text { if Cind }>\text { Cthresh then }
$$$$
\text { is_stru }=1 . \quad \text { (Estrutura) }
$$$$
\text { else }
$$$$
\text { is_stru }=-1 . \quad \text { (Ruído) }
$$$$
\text { end if }
$$

\section{Algoritmo 2: Contribuição}

$D_{p}=P_{1}-P_{2}$

$M_{p}=\max \left(\frac{\operatorname{mean}(P 1)+\operatorname{mean}(P 2)}{2}, \frac{1}{n \times n}\right)$

$\operatorname{ctri1}=\operatorname{cov}\left(P_{1} ; D_{p}\right)$

$\operatorname{ctri2}=\operatorname{cov}\left(P_{2} ;-D_{p}\right)$

$$
\text { ctri }=\frac{c t r i 1-c t r i 2}{M_{p}}
$$

$$
\operatorname{cov}(x, y)=\frac{(x-\operatorname{mean}(x))^{T}(y-\operatorname{mean}(y))}{n^{2}-1}
$$

Onde x e y são patches vetorizados de tamanho $n^{2} \times 1$, s 1 e s2 são valores singulares de $\mathrm{G}, \mathrm{C}_{\text {thresh }}$ é um threshold constante para binarizar $\mathrm{C}_{\text {ind, }}, \mathrm{P}_{1}$ e $\mathrm{P}_{2}$ são dois patches de tamanho $n \times n$ das imagens $f$ e $g$. 


\subsubsection{Comparison Texture Quality (CTQ)}

Em [45] o autor defende ainda que o método CQ sofre de um problema de compensação ao não levar em consideração informações relativas à textura e para isso desenvolveu o método CTQ para corrigir este caso, adicionando um novo módulo, o de Compensação de Textura, ao pipeline definido anteriormente.

$$
C T Q I=\frac{1}{M N} \sum_{i, j=\left(\frac{n}{2}\right):\left(M-\frac{n}{2}\right)} \operatorname{CTQ}(i, j)
$$

$C T Q=i s_{\text {stru }} \cdot$ ctri $\cdot$ weight

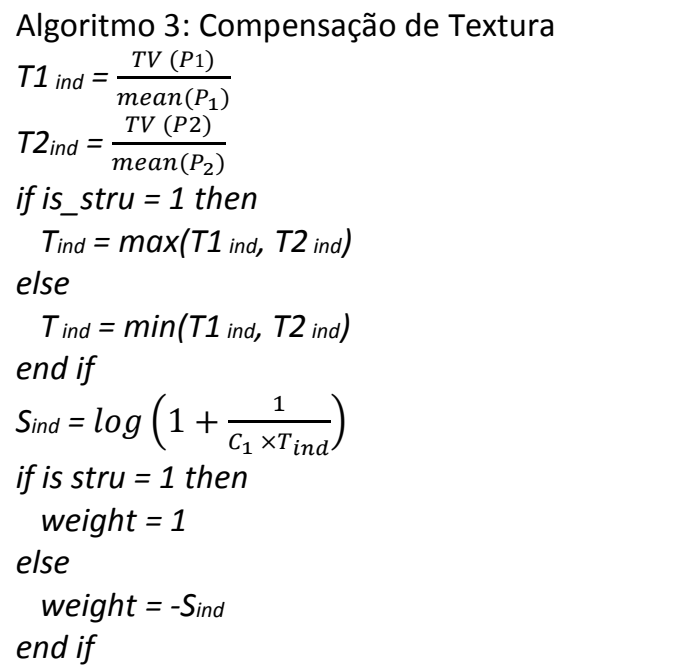

Onde TV(P) é a variação total no patch original, mean $(\mathrm{P})$ a média do patch original, $\mathrm{T}_{\text {ind }}$ a complexidade da textura percebida.

\subsubsection{Sem Referência}

No último grupo estão as métricas que não precisam de referência alguma para atribuírem uma pontuação ao resultado de um filtro, usando somente a própria imagem filtrada como parâmetro de avaliação. Dado que imagens naturais tendem a não possuir variações bruscas de direção de gradiente e intensidades elevadas somente em fronteiras entre estruturas distintas, estas métricas se baseiam na presunção de uniformidade de direcionamento da matriz de gradientes para uma região da imagem.

\subsubsection{MetricQ (Q)}

Nessa métrica, dois índices refletem a qualidade do patch local: O Índice de Conteúdo de Imagem (S), que reflete a proeminência de estruturas em um patch local e o Índice de Coerência (R), usado para determinar se o patch local é dominado por ruído. Q pode ser interpretado, portanto, como uma média dos índices de estrutura dos patches locais que possuem um conteúdo relevante[61].

$$
Q=\frac{1}{M N} \sum_{i, j: R(i, j)>\tau} S(i, j)
$$


$S=s_{1} \frac{s_{1}-s_{2}}{s_{1}+s_{2}}$
$R=\frac{s_{1}-s_{2}}{s_{1}+s_{2}}$

Onde $\tau$ é um threshold para decidir se o patch local é dominado por ruído, $R(i, j)>\tau$ é um teste para verificar se os patches anisotrópicos encontrados na imagem de entrada satisfazem a condição de cuído.

Os autores reiteram ainda que, como esta métrica só leva em consideração regiões estruturadas (patches anisotrópicos), ela não funciona devidamente em situações onde o ruído aparece somente em regiões lisas (patches isotrópicos) e ainda que esta métrica mede o conteúdo da imagem ao invés da qualidade relativa da imagem.

\subsection{Categorias de Limites das Métricas}

As métricas selecionadas não necessariamente possuem os mesmos intervalos de pontuação, como por exemplo, a métrica SNR possui um limite teórico de infinito para seu melhor resultado, apesar de um limite ter sido apresentado na seção anterior como um passa alta, ao passo que a métrica FoM e COC têm suas pontuações contidas no intervalo $[0,1]$. Então para poder atribuir uma pontuação consistente de forma que métricas diferentes pudessem ser comparadas entre si, uma normalização foi necessária e esta definição de limites práticos resultou em três grupos de avaliação, como visto na Tabela 4.1.

Como os limites teóricos costumam acontecer em casos extremos, geralmente comparando uma imagem composta somente por pixels brancos com uma imagem composta somente por pixels pretos, estes não são úteis para comparação de resultados uma vez que os patamares criados por essa abordagem iriam diferenciar somente os resultados bons dos ruins e não ranquearia eficientemente os resultados bons entre si, portanto não pontuando corretamente um filtro que atingiu o melhor resultado daquele que atingiu, por exemplo, o quinto melhor resultado.

A determinação dos limites práticos de forma de forma empírica nos permite diminuir o intervalo de normalização dos resultados, fornecendo assim pontuações mais sensíveis às variações de valores.

Por esse motivo foi necessário fixar limites práticos para cada métrica. Quando os valores de fato encontrados pelas métricas são além dos limites práticos estabelecidos, estes atuam como um threshold a partir do qual os valores recebem uma pontuação mínima. Isto não é um problema no contexto deste trabalho, uma vez que estamos buscando somente os melhores resultados, e não a classificação exata de cada filtro em relação a uma métrica. 


\begin{tabular}{|c|c|c|c|c|c|c|}
\hline \multirow[t]{2}{*}{ Métricas } & \multicolumn{2}{|c|}{ Limites Teóricos } & \multicolumn{2}{|c|}{ Limites Práticos } & \multirow{2}{*}{$\begin{array}{c}\text { Melhor } \\
\text { Valor }\end{array}$} & \multirow[t]{2}{*}{ Grupo } \\
\hline & Inferior & Superior & Inferior & Superior & & \\
\hline$A D$ & 0 & 255 & 0 & 32 & 0 & Zero \\
\hline MSE & 0 & $255^{2}$ & 0 & $2^{12}$ & 0 & Zero \\
\hline M3 & 0 & $255^{2}$ & 0 & $2^{6}$ & 0 & Zero \\
\hline M4 & 0 & $255^{2}$ & 0 & $2^{6}$ & 0 & Zero \\
\hline$S N R$ & 0 & $10^{6}$ & 0 & 100 & 100 & Min-Max \\
\hline UQI & -1 & 1 & -1 & 1 & 1 & Min-Max \\
\hline SSIM & -1 & 1 & -1 & 1 & 1 & Min-Max \\
\hline$S C$ & 0 & $\mathrm{MN} \cdot 25^{2}$ & 0 & 5 & 1 & Centered \\
\hline$N K$ & 0 & 255 & 0 & 5 & 1 & Centered \\
\hline$M D$ & 0 & 255 & 0 & 255 & 0 & Zero \\
\hline LMSE & 0 & $(\mathrm{MN} \cdot 255 \cdot 2)^{2}$ & 0 & 4 & 0 & Zero \\
\hline$N A E$ & 0 & $M N \cdot 255$ & 0 & 1 & 0 & Zero \\
\hline $\mathrm{COC}$ & 0 & 1 & 0 & 1 & 1 & Min-Max \\
\hline FOM & 0 & 1 & 0 & 1 & 1 & Min-Max \\
\hline SSI & 0 & $255^{2}$ & 0 & 1 & 0 & Zero \\
\hline SMPI & 0 & $2 \cdot 255^{2}$ & 0 & 5 & 0 & Zero \\
\hline$E S I$ & 0 & $M N \cdot 255$ & 0 & 100 & 100 & Min-Max \\
\hline Beta & -1 & 1 & -1 & 1 & 1 & Min-Max \\
\hline$C Q$ & - & - & -4 & 4 & 0 & Centered \\
\hline CTQ & - & - & -4 & 4 & 0 & Centered \\
\hline MetricQ & - & - & 0 & 100 & 100 & Min-Max \\
\hline
\end{tabular}

Tabela 4.1: Limites teóricos e práticos das métricas, bem como seus melhores resultados e o grupo a qual pertencem.

\subsubsection{Min-Max}

As métricas contidas no grupo Min-Max são aquelas que após serem normalizadas ficam com seus valores entre 0 ou -1 , no pior caso, e 1 , no melhor caso. FOM e ESI variam entre 0 e 1 , ao passo que Beta, COC, UQI e SSIM variam entre -1 e 1 . Entretanto estas últimas, com exceção do Beta, podem ser usadas em módulo, uma vez que para o COC o valor -1 também implicaria em uma correlação total entre as imagens, enquanto UQI e o SSIM consideram além da correlação, a proporção entre as médias, sendo o valor -1 também é um indicativo forte de correlação.

Não conseguimos encontrar referências ou estabelecer os limites teóricos para a métrica MetricQ, mas ao ser analisado empiricamente, contatou-se que este pertencia à categoria Min-Max.

O SNR é um caso à parte uma vez que seu limite superior teórico (melhor caso) é infinito, com duas imagens idênticas, e seu limite inferior teórico (pior caso) é zero, com duas imagens opostas, uma toda branca e outra toda preta, por exemplo. Ambos os limites implicam em problemas para a comparação de resultado uma vez que, ao contrário dos outros grupos, o melhor caso aqui não possui um valor 
específico a ser atingido e a pontuação precisa ser consistente ao longo de diferentes testes.

Visando resolver este problema, adotou-se por usar como limite superior prático o valor de 100 e um limite inferior prático de 1 , considerando todos os casos com mais ruído que sinal como a pior pontuação. Essas mudanças, no entanto, não são suficientes para a comparação, uma vez que na literatura valores de SNR acima de 50 já são consideradas imagens sem ruído.

Para simplificar a pontuação, a comparação entre os resultados do SNR foi feita com a seguinte formula:

$$
1-\frac{1}{S N R}
$$

Esta fórmula normaliza o espaço dos resultados do SNR entre 0, quando o SNR possuir valor 1 (pior caso), e $\sim 1$, quando o SNR possuir valor $10^{6}$ (melhor caso). Apesar desta fórmula aglutinar os melhores resultados com pontuações semelhantes, ela resulta em uma pontuação melhor para o filtro que for mais consistente do que para aquele que tiver um melhor resultado isolado.

\subsubsection{Centered}

No grupo Centered estão as métricas em que seus melhores resultados possuem valor 1, mas elas podem atingir tanto valores superiores ou inferiores que ele, sendo por isso centradas em 1. Para ambas as métricas SC e NK, é possível provar que o limite inferior teórico é 0 ao se ter uma imagem preta como entrada, ao passo que seu limite superior teórico é infinito ao se ter também uma imagem preta como entrada. Entretanto este valor não é útil para uma comparação de performance entre as métricas e por isso foi necessário definir um limite superior prático em 5 para ambas, uma vez que este valor já significa uma diferença grande entre as imagens de entrada, tendo este valor sido encontrado por amostragem.

Não foi possível também estimar os limites teóricos das métricas CQ e CTQ, apesar de que se notou que seu comportamento é similar ao das outras métricas contidas no grupo Centered, sendo assim estes foram definidos empiricamente.

\subsubsection{Zero}

Todas as métricas contidas neste grupo têm seus melhores resultados fixados em 0 , entretanto seus piores casos são teóricos.

A métrica MSE possui um limite superior teórico em $255^{2}$ (65.025) ao se ter uma das imagens de entrada totalmente branca e outra totalmente preta. Seu limite superior prático foi definido em $2^{12}$ (4096), o que significa que a diferença média de cada pixel da imagem seria de 64 , ou seja, de $25 \%$ da amplitude máxima possível em uma imagem em tons de cinza codificada com um byte por pixel. Vale notar que a métrica RMSE é uma norma-L de ordem 2, o que faz com que a MSE seja similar a uma norma-L. Esta métrica foi escolhida em detrimento da RMSE, uma vez que a variabilidade da MSE é superior à da RMSE (definida como a raiz quadrada de MSE), sendo assim mais sensível a diferenças e, portanto, possuindo pontuações mais esparsas.

Ambas as métricas M3 e M4 possuem um limite superior teórico em 255 ao se usar uma imagem completamente preta e outra completamente branca na comparação. Seu limite superior prático foi definido em $2^{6}(64)$, o que corresponde 
à raiz quadrada do limite superior empírico do MSE (4096), que possui unidade de medida quadrática em relação a esta. A similaridade entre essas três métricas é que são normas- L de ordens diferentes, onde o RMSE é de ordem 2, o M3 de ordem 3 e o M4 de ordem 4.

AD também é uma norma-L, porém de $1^{\text {a }}$ ordem (distância de Hamming), possuindo um limite superior teórico em 255 pelos mesmos motivos apresentados anteriormente para as métricas M3 e M4. Por serem similares, seria plausível definir seu limite superior prático também em 64, porém por ser menos sensível à variabilidade da diferença entre as imagens, um limite prático em 32 resultou em uma pontuação mais esparsa que o limite em 64 forneceria.

A métrica MD possui um limite superior teórico definido em 255 , onde para que isso aconteça basta que um único pixel branco de uma imagem se torne preto, ou vice-versa. Como parece plausível que alguma diferença entre um par de pixels em uma imagem resulte em um valor alto, decidiu-se por adotar o limite superior teórico como o limite superior prático desta métrica.

A métrica NAE possui um limite superior prático de infinito para o caso de uma das imagens ser totalmente preta, ou de M.N.255 para o caso desta imagem ser toda preta com a exceção de um único pixel com valor 1 . Seu limite superior prático foi definido em 1, uma vez que para atingir este valor seria necessário que, para cada pixel de uma imagem de entrada, o valor do mesmo pixel na outra imagem fosse o dobro ou nulo, o que significa que ambas as imagens já seriam bem diferentes entre si, mas ainda apresentando uma margem grande o suficiente para que imagens pouco diferentes possuam pontuações diferentes de zero.

Por fim na LMSE definiu-se um limite prático superior em 4 por amostragem.

\subsection{Tipos de Pontuação}

Uma vez com as métricas e seus intervalos bem definidos, foi necessário ainda criar uma maneira de avaliar o resultado de um filtro perante o conjunto de métricas em si, uma vez que métricas diferentes podem avaliar diferentes aspectos de uma imagem e o melhor filtro deve possuir pontuações consistentes na maior quantidade de métricas possível. Quatro novos tipos de avaliação foram então desenvolvidos, cada um dividido ainda em Light e Weighted:

1. All Score

2. Full Reference Score (FR) - considera apenas as métricas de referência completa.

3. Reduced Reference Score (RR) - considera apenas as métricas de referência reduzida.

4. No Reference Score (NR) - considera apenas as métricas que não utilizam referência.

Essas novas avaliações aglomeram os resultados das métricas e verificam a consistência de uma imagem ao longo de cada tipo de avaliação, enquanto as classificações Light e Weighted atribuem pesos para cada métrica, com a Light atribuindo o mesmo peso para todas as métricas e a Weighted atribuindo pesos diferentes. Vale ressaltar que como apenas uma métrica sem referência foi usada, a MetricQ, o resultado do NR Light e Weighted são iguais. 
A avaliação All Score leva em consideração todas as formas de pontuação usadas ao longo dos testes, enquanto nas seguintes cada uma leva em consideração somente as métricas com seu próprio tipo de referência.

O sistema Light e Weighted foi necessário uma vez que testes preliminares apontaram que imagens com bons resultados visuais frequentemente tinham pontuações elevadas em determinadas métricas, portanto com o intuito de direcionar os resultados obtidos e assim de premiar filtros que tivessem um melhor resultado nessas métricas, pesos no intervalo $(0,1]$ foram arbitrados para cada forma de avaliação e são apresentados na Tabela 4.2.

\begin{tabular}{|l|l|l|l|l|l|l|l|l|l|l|l|}
\hline Pesos & $\mathbf{1}$ & $\mathbf{0 , 7 5}$ & $\mathbf{0 , 5}$ & $\mathbf{0 , 5}$ & $\mathbf{0 , 7 5}$ & $\mathbf{0 , 2 5}$ & $\mathbf{0 , 2 5}$ & $\mathbf{0 , 2 5}$ & $\mathbf{0 , 2 5}$ & $\mathbf{0 , 2 5}$ & $\mathbf{1}$ \\
\hline Métricas & MSE & SNR & M3 & M4 & UQI & SSIM & AD & SC & NK & MD & LMSE \\
\hline Pesos & $\mathbf{0 , 2 5}$ & $\mathbf{1}$ & $\mathbf{1}$ & $\mathbf{1}$ & $\mathbf{1}$ & $\mathbf{0 , 2}$ & $\mathbf{0 , 8}$ & $\mathbf{1}$ & $\mathbf{0 , 2 5}$ & $\mathbf{0 , 7 5}$ \\
\hline Métricas & NAE & COC & FOM & MetricQ & BETA & SSI & SMPI & ESI & CQ & CTQ \\
\hline
\end{tabular}

Tabela 4.2: Os pesos adotados para cada métrica para o sistema de avaliação Weighted. 


\section{Escolha dos parâmetros dos Filtros 2D}

Uma vez com o método geral de avaliação de filtros bem definido, foi necessário escolher um intervalo de parâmetros individuais de cada filtro para a optimização de resultados. De acordo com a notação [valor inicial : incremento iterativo : valor final], um exemplo para esta otimização pode ser feito levando em conta o filtro Trilateral, que possui quatro parâmetros:

- Número total de Iterações: Com o intervalo $[1,16]$ e incrementos unitários, $[1: 1: 16]$

- Sigma: Com o intervalo $[1,16]$ e incrementos unitários, [1: $1: 16]$.

- Épsilon: Com o intervalo $[0,1]$ e incrementos de $1 / 16,[0: 1 / 16,1]$.

- Beta: Com o intervalo $[0.15,0.45]$ e incrementos de $0.15,[0.15$ : $0.15: 0.45]$

Cada possível combinação dos valores dos parâmetros desse filtro resulta em uma nova imagem, em que ao final desse teste 13.056 imagens teriam sido geradas, precisando assim de um grande esforço computacional.

A etapa de minimização e optimização dos parâmetros foi desenvolvida com o uso do seguinte algoritmo:

1. Para cada filtro.

a. Para cada arquivo de teste.

i. Para cada parâmetro.

1. Fixar este parâmetro.

2. Variar todos os outros.

3. Guardar o melhor resultado.

b. Gerar para cada tipo de parâmetro um gráfico dos resultados guardados.

c. Verificar quais valores para cada intervalo de cada parâmetro resultaram em uma melhor média de pontuações.

Todos os filtros foram examinados nos mesmos 18 casos de teste, que são imagens fetais obtidas por equipamentos de ultrassom da General Eletric de diferentes modelos Voluson, com diferentes transdutores usados. Os exames fetais variam no período gestacional de 7 a 15 semanas e as imagens usadas são fatias $2 \mathrm{D}$, de diferentes eixos, de 8 exames de ultrassom 3D volumétrico. Apesar dos testes serem feitos somente sobre imagens fetais, não existe qualquer restrição dos métodos discutidos de serem usados em outros tipos de exames de ultrassom. 


\subsection{Análises por gráfico}

Um exemplo da análise por gráfico pode ser visto na Figura 5.1, onde cada linha presente no gráfico representa uma imagem de teste, com todas as 18 usadas. Este gráfico de exemplo representa a melhor pontuação em cada imagem para um mesmo parâmetro de um único filtro, onde é possível reparar que apesar dos 20 valores usados no conjunto $[0.01: 0.1: 2]$, apenas o conjunto $[0.01: 0.1: 0.51]$ (o primeiro quadrante da figura) já seria suficiente, sendo por si só uma diminuição de $70 \%$ do total de imagens geradas para este filtro. Cada parâmetro de cada filtro resultou em um gráfico semelhante.

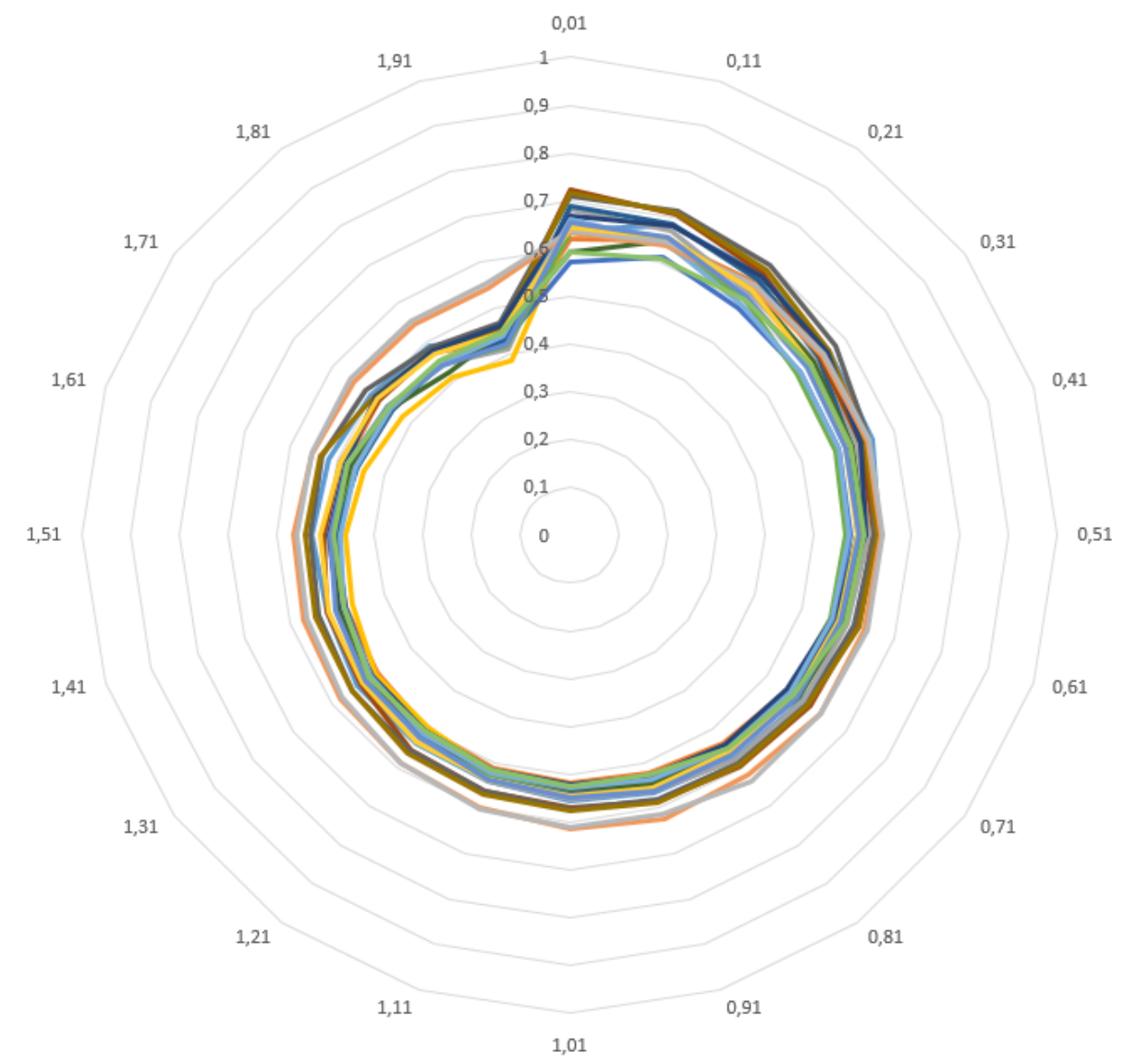

Figura 5.1: Melhores resultados de cada imagem para cada variação de um único mesmo parâmetro. Os valores em torno do círculo representam a variação de todos os valores usados neste parâmetro, enquanto os valores na vertical representam a melhor pontuação obtida em uma métrica de agrupamento (FR para esta imagem). As linhas coloridas representam os 18 casos de teste abordados.

Os gráficos gerados na etapa anterior, entretanto, não são definitivos para a escolha dos melhores parâmetros, uma vez que exibem apenas a pontuação máxima de cada filtro com um dado parâmetro fixado, sendo ainda necessário verificar o comportamento individual de cada filtro para cada imagem para que a consistência de seus parâmetros pudesse ser atestada.

Na Figura 5.2 podemos perceber como a etapa anterior não é suficiente para uma boa indicação dos parâmetros de cada filtro. À esquerda desta figura temos um gráfico gerado de acordo com o algoritmo citado anteriormente, onde é possível notar que o conjunto [3:2:9] contém resultados ligeiramente melhor para os 
parâmetros deste filtro. Entretanto, à direita desta figura temos os gráficos individuais de 3 das 18 imagens de testes para este filtro.

Cada um destes novos gráficos representa a pontuação obtida em cada variação dos parâmetros deste filtro, sendo justamente esse o maior diferencial em relação ao gráfico anterior, que só apresentava o máximo de cada imagem.

Com essa visualização podemos perceber que por vezes a maior pontuação obtida por um parâmetro é na verdade um outlier, conforme apontado pelas setas em cada gráfico, e não sendo assim consistentemente uma boa escolha, como é o caso dos valores 7 e 9 desse parâmetro, que só são consistentes em resultados que tiveram um baixo desempenho. Apesar disso, os valores 3 e 5 consistentemente apresentam desempenhos próximos ao máximo observado e são assim escolhas melhores e mais seguras.

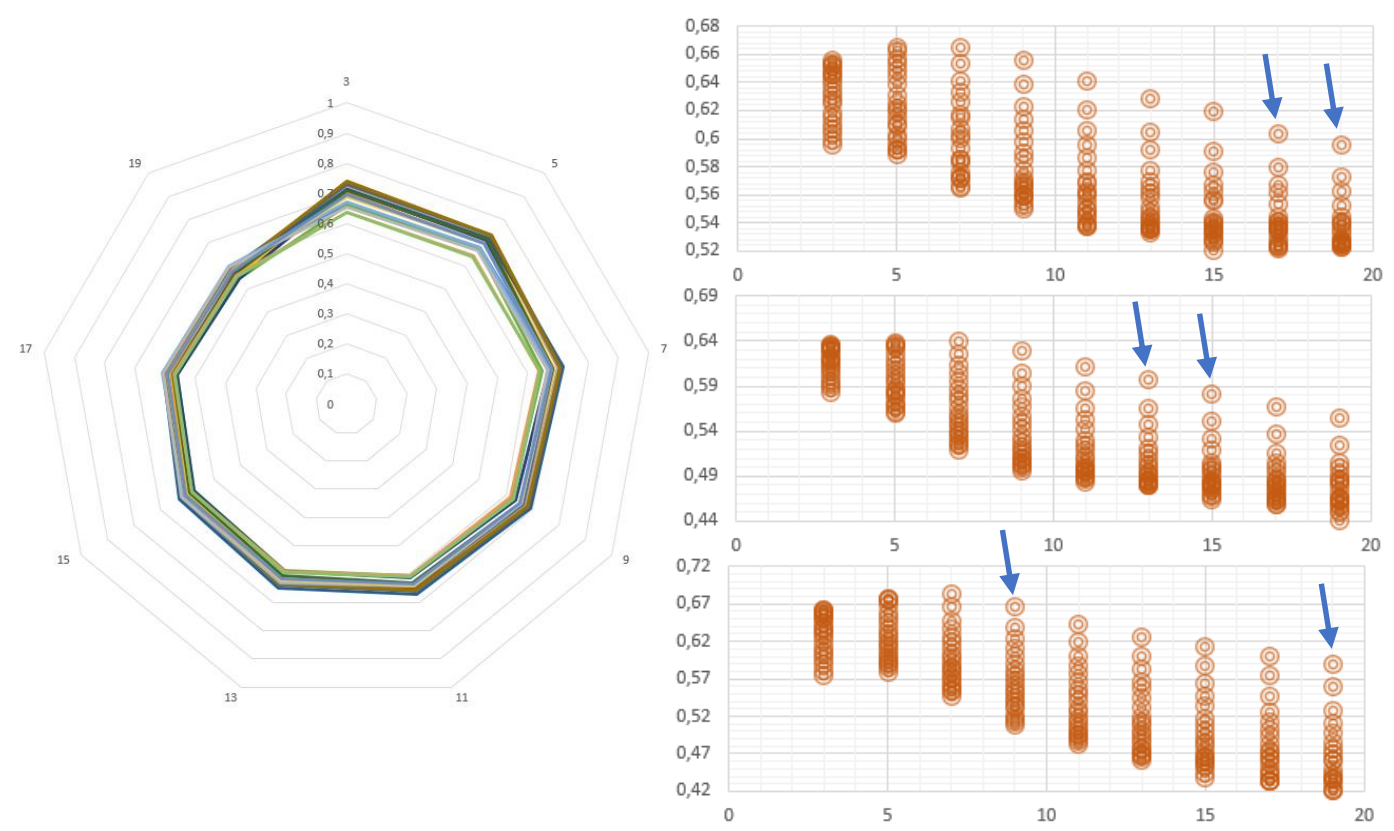

Figura 5.2: À esquerda: As melhores pontuações de todas as variações de um mesmo parâmetro, conforme visto na figura anterior. À direita: $\mathrm{O}$ eixo vertical representa o intervalo de pontuações atingido por esta configuração de parâmetros, enquanto o eixo horizontal contém a variação de um único parâmetro, no caso [3: $2: 19]$. Os anéis significam as pontuações obtidas ao fixar o parâmetro visto no eixo vertical e variar os outros parâmetros deste filtro. Três gráficos são apresentados à direita para apresentar o comportamento médio obtido por este filtro, obtendo seus melhores resultados com os valores 3 e 5 neste parâmetro. As setas representam alguns dos elementos individuais tidos como outliers, presentes em cada teste.

A escolha da visualização dos resultados em anéis, nos gráficos, foi feita justamente para que quando diversos anéis se interceptassem, uma linha preenchida começaria a se formar, indicando uma consistência na escolha dos parâmetros ao apresentar uma concentração na distribuição de elementos.

Visando simplificar a análise dos gráficos apresentados na etapa anterior, optou-se por combiná-los em uma única visualização, aplicando uma transparência nos pontos observados para que a sobreposição de diversos pontos pudesse ser percebida, indicando assim com mais clareza onde os parâmetros são mais consistentes. 
Analisando a Figura 5.3 é possível observar que os valores 3 e 5 deste parâmetro possuem as maiores pontuações sendo ainda o resultado mais consistente, visto que no gráfico eles possuem as linhas mais escuras.

Apesar dos esforços para criar representações que indicassem porque um resultado deve ser escolhido em detrimento de outros, todas formas de análise apresentadas até então sofrem de um mesmo problema: ao se trabalhar com muitos dados, as visualizações por vezes se tornam exaustivas ou inconclusivas, seja por existirem muitas interseções em um mesmo gráfico ou pela falta delas, resultando em um gráfico com dados esparsos.

O método determinante para a escolha do melhor conjunto de parâmetros foi o de clusterização por $k$-Means [62].

\section{2. $k$-Means}

O k-means é um processo iterativo que particiona os dados de entrada em $k$ grupos diferentes, onde cada dado só pode estar em exatamente um dos grupos, também conhecidos como clusters.

Seu pseudocódigo pode ser visto a seguir:

1. Determinar os $k$ centros iniciais dos clusters por $k$ means++[63].

2. Calcular a distância de todos os elementos de entrada para cada centroide.

3. Optou-se por determinar individualmente os elementos para um cluster diferente caso isso diminua a soma das distâncias entre pontos e centroides de um mesmo cluster.

4. Calcular a média dos elementos em cada cluster para obter $k$ novas posições de centroides.

5. Repetir os passos de 2 a 4 até que nenhum elemento troque de cluster ou que o número máximo de interações ocorra.

O algoritmo de $k$-means++ é usado para diminuir o tempo de processamento do $k$-means ao agilizar a convergência apresentada no passo 3 , conseguindo ainda aumentar a qualidade da solução final.

Um problema comum encontrado ao se usar o $k$-means é que os elementos podem ser agrupados em clusters diferentes dependendo do chute inicial apresentado no passo 1. Aqui esse problema é contornado ao repetir o algoritmo diversas vezes, retornando apenas os resultados da solução com a menor soma de distâncias dos elementos para o centroide intra-clusters. 


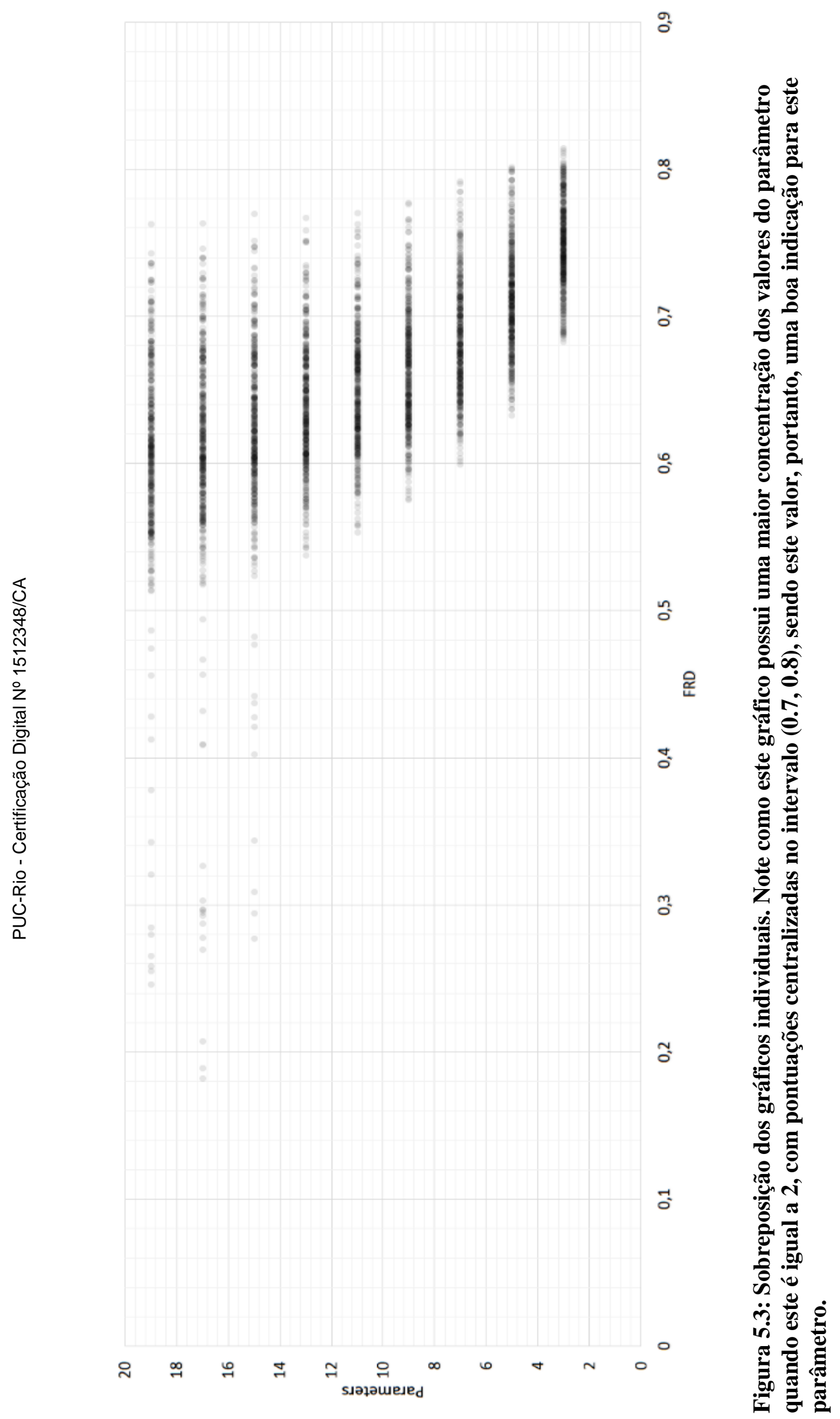


Outra especialização adotada foi a de usar o conceito de cityblock como medida de distância ao invés da distância euclidiana quadrática. Essa escolha foi feita uma vez que o uso da distância euclidiana infere que exista não só uma correlação entre os eixos dos dados apresentados como que os eixos estejam em uma mesma escala, o que em ambos os casos não podem ser verificados neste trabalho. Os valores entre parâmetros não possuem qualquer relação entre si e seus valores podem tanto variar entre 0 e 1 quanto entre 1 e 255 , entretanto vale notar que os valores de performance possuem sim uma correlação com os parâmetros usados apesar desse motivo sozinho não justificar o uso da distância euclidiana quadrática.

Apesar de fornecer resultados melhores que os vistos anteriormente, este método precisa de uma boa escolha do valor $k$ para que sua performance seja otimizada. A escolha deste valor se deu de duas maneiras diferentes:

1. Critério de Gap [64]

2. kSqrt: $\sqrt[2]{\frac{\text { totalElements }}{2}}[65]$

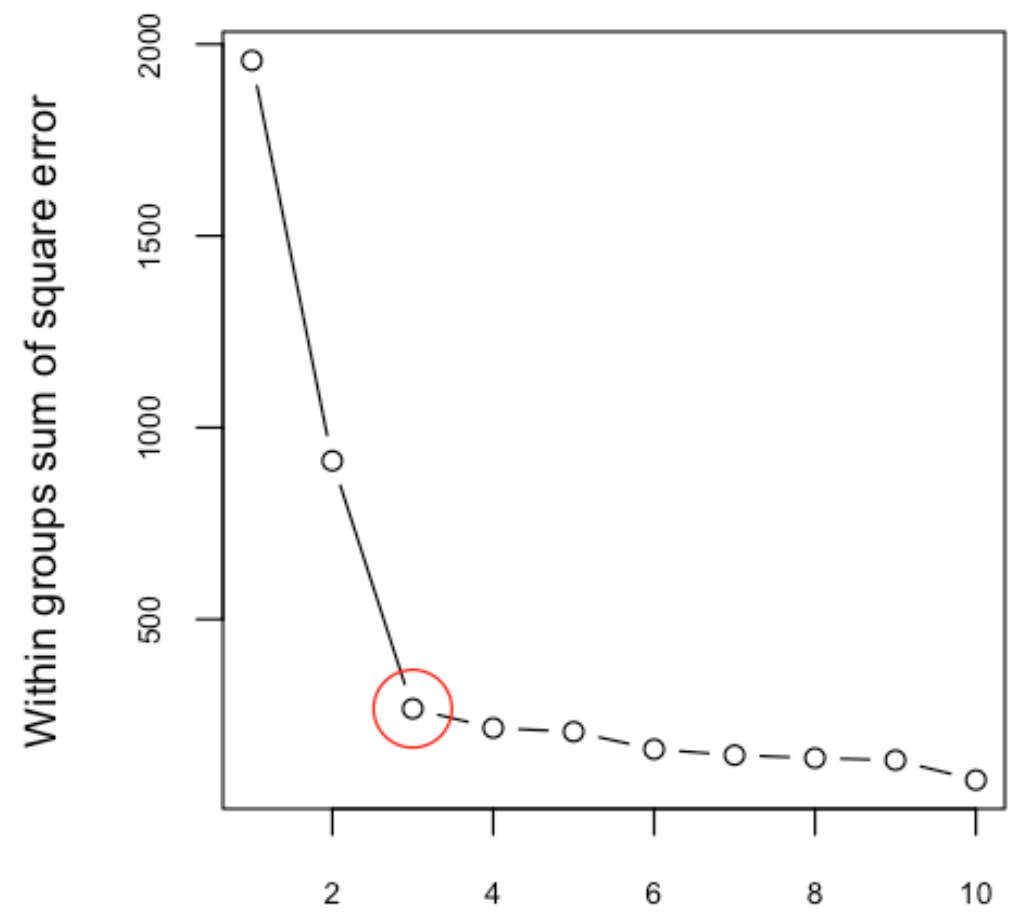

\section{Number of Clusters}

Figura 5.4: Representação do método do "cotovelo" para determinar a melhor escolha do número total de clusters a serem usados no $k$-means [66]. Nesta imagem o valor de $k=3$, circundado em vermelho, representa o total de clusters que melhor descreve os dados utilizados.

Um método comum para a determinação do total de clusters usados é o do "cotovelo" ("elbow" em inglês), onde diferentes valores são testados e é criado um gráfico do total de clusters usados e da soma dos erros quadráticos (SSE) entre os pontos do cluster e sua média, como pode ser visto na Figura 5.4. A ideia por trás deste método é que um menor SSE implica em um melhor resultado, entretanto a taxa com que o SSE varia não é constante e tende a convergir em um determinado 
ponto, o valor de k escolhido será aquele que começar a apresentar um SSE com rendimentos decrescentes.

O critério de Gap formaliza a abordagem do "cotovelo" ao estimar o total de clusters na solução com o maior espaço (gap) local ou global dentro de uma tolerância e é definido pela seguinte fórmula:

$$
\begin{aligned}
& \operatorname{Gap}_{n}(k)=E_{n}^{*}\left\{\log \left(W_{k}\right)\right\}-\log \left(W_{k}\right) \\
& W_{k}=\sum_{r=1}^{k} \frac{1}{2 n_{r}} D_{r \prime}
\end{aligned}
$$

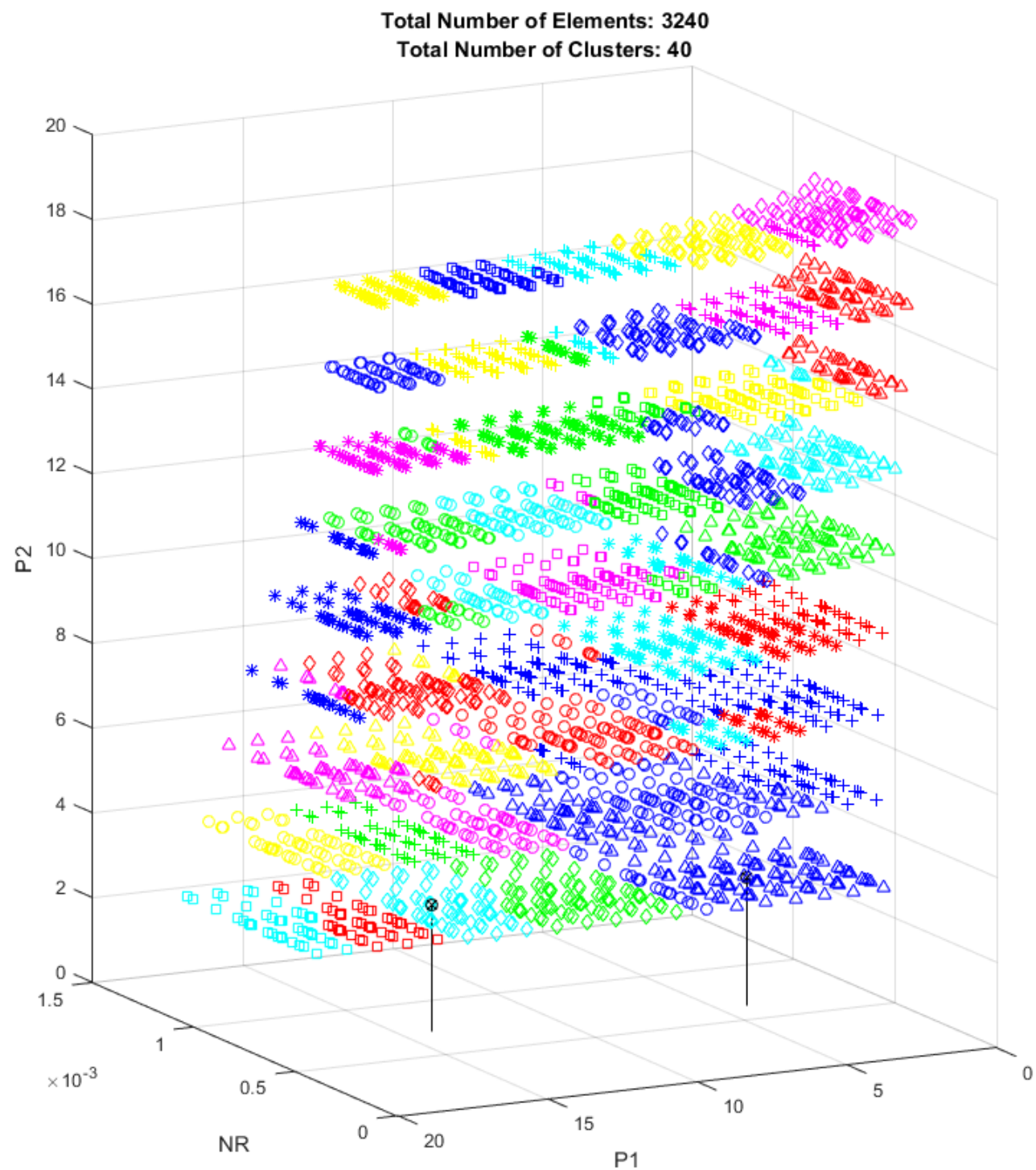

Figura 5.5: Visualização do resultado da aplicação do $k$-means para todos os casos de teste e com somente o filtro DsFhomo, neste exemplo com apenas dois parâmetros (P1 e P2) e para o método de avaliação NR. A linha preta à direita representa o cluster com maior cardinalidade relativa, enquanto a linha preta à esquerda representa o cluster de maior performance. Cada combinação de cor e símbolo representam idealmente um cluster diferente, entretanto como existe um limite de 6 cores e 6 símbolos nesta representação e 40 clusters foram usados, quatro combinações estão repetidas. 
Onde $\mathrm{n}_{\mathrm{r}}$ é o total de pontos em um cluster $r$, e $\mathrm{D}_{\mathrm{r}}$ é a soma das distâncias entre pares para todos os pontos no cluster $r$. O valor esperado $E_{n}^{*}\left\{\log \left(W_{k}\right)\right\}$ é determinado pela amostragem de Monte Carlo a partir de uma distribuição de referência.

Este critério, entretanto, é computacionalmente custoso e para grandes datasets o segundo método para determinar o valor de $k$, chamado neste trabalho de kSqrt, é usado, aplicando a raiz quadrada da metade do total de elementos do cluster, sendo este, portanto um método empírico. O threshold adotado para o uso do Critério de Gap ou do kSqrt é que filtros que tenham obtido mais de 550 variações de parâmetros em cada teste, ou seja, mais de 10000 imagens resultantes para todos os testes, devem usar o kSqrt, caso contrário o Critério de Gap deve ser usado.

Um exemplo da aplicação do k-means com os métodos acima pode ser visto na Figura 5.5. Aqui dois tipos de análise para cada tipo de pontuação agregada podem ainda ser realizados: levando em consideração os grupos com maior cardinalidade relativa, ou seja, o cluster com maior número de elementos, e o grupo com centroide de maior performance. A primeira análise é usada para encontrar o grupo de parâmetros de um filtro que teve uma avaliação mais consistente ao longo de todos os testes, enquanto a segunda visa selecionar somente os melhores candidatos, com suas visualizações presentes na Figura 5.6.
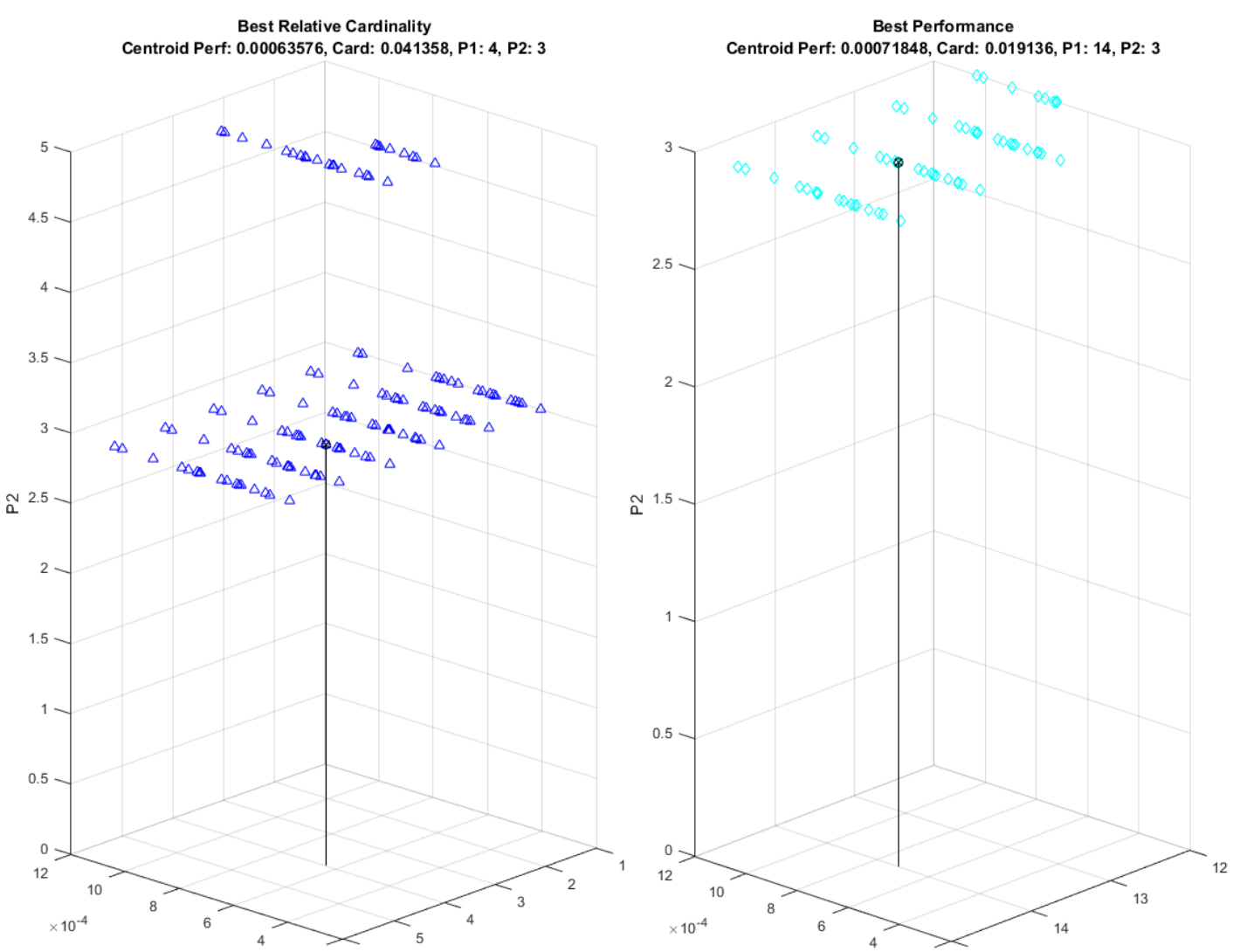

Figura 5.6: Representação dos clusters de maior Cardinalidade Relativa e melhor Performance, respectivamente. Neste exemplo o DsFhomo continua sendo usado assim como somente a métrica NR é avaliada para simplificação. Perf representa o desempenho obtido pelo centroide deste cluster na métrica NR, enquanto Card representa a Cardinalidade Relativa atingida. P1 e P2 são os parâmetros utilizados por este filtro. 


\section{Avaliação}

\subsection{Setup de testes}

Todos os testes foram feitos no ambiente Matlab R2015a, com exceção do filtro Trilateral que teve sua implementação em $\mathrm{C}++$, e apesar de diferentes computadores terem sido utilizados para diminuir o tempo de cálculo de algumas das avaliações propostas, o computador principal foi um Intel Core i7-5930K, com 32GB DDR4 e placa de vídeo GeForce GTX 980 Ti em SLI.

Diversas etapas das avaliações foram calculadas em várias threads, como os cálculos dos filtros e métricas e a clusterização de resultados, entre outros, com alguns casos ainda sendo calculados em GPU, como o cálculo da métrica Figure of Merit (FOM).

\begin{tabular}{|c|c|c|}
\hline Filtros & Parâmetros & Total \\
\hline \multicolumn{3}{|l|}{ Lineares } \\
\hline DsFlsmv 3.1.1.1 & ITL: [1:1:20]; SWS: [3:2:19]; & 180 \\
\hline DsFwiener2 3.1.1.2 & ITL: [1:1:20]; SWS: [3:2:19]; & 180 \\
\hline DsFlsminsc 3.1 .2 & ITL: [1:1:20]; SWS: [3:2:9]; Edge Detector: [0:1:4]; & 400 \\
\hline \multicolumn{3}{|l|}{ Não Lineares } \\
\hline DsFmedian 3.2.1 & ITL: [1:1:20]; SWS: [3:2:19]; & 180 \\
\hline DsFca 3.2.2 & ITL: [1:1:20]; SWS: [3:2:19]; & 180 \\
\hline DsFls 3.2 .3 & ITL: [1:1:20]; SWS: [3:2:19]; & 180 \\
\hline DsFlecasort 3.2.4 & ITL: [1:1:20]; SWS: [3:2:19]; & 180 \\
\hline DsFhomog 3.2 .5 & ITL: [1:1:20]; SWS: [3:2:19]; & 180 \\
\hline DsFgf4d 3.2.6 & ITL: [1:1:20]; SWS: [3:2:19]; & 180 \\
\hline DsFhomo 3.2 .7 & ITL: [1:1:20]; SWS: [3:2:19]; & 180 \\
\hline DSDN 3.2.8 & $\begin{array}{l}\text { Noise Type: [1:1:3]; Fusing: [1:1:2]; Window Type: [1:1:2]; } \\
\text { Symmetry Type: [1:1:3]; }\end{array}$ & 180 \\
\hline SADCTD 3.2 .9 & Sigma Limit: [1:1:2]; Sigma Delta: [0.025: 0.025:1]; & 80 \\
\hline \multicolumn{3}{|l|}{ DIFUSÃO } \\
\hline DsFad 3.3.1 & ITL: [1:1:10]; Kappa: [1:1:16]; Lambda: [0:0.05:0.1] ; Option: [1:1:2]; & 960 \\
\hline DsFsrad 3.3.2 & ITL: [1:1:20]; Lambda: [0.01: 0.1:2]; ROI; & 400 \\
\hline DsFlndif 3.3 .3 & $\begin{array}{l}\text { ITL: [1:1:8]; Lambda: [1:1:6]; Sigma: [0.01:0.5:2.41]; Difusivity Speed: } \\
\text { [4:3:7]; Step Size: [1:3:4]; }\end{array}$ & 960 \\
\hline Bilateral 3.3.4 & ITL: [1:1:8]; Sigma S: [2:1:8]; Sigma R: [5:4:49]; & 672 \\
\hline Trilateral 3.3 .5 & $\begin{array}{l}\text { ITL: [1:1:8]; Sigma C: [1:1:8]; Epsilon: [0.01: 0.07:0.36]; Beta: } \\
\text { [0.15:0.15:0.9]; }\end{array}$ & 2304 \\
\hline \multicolumn{3}{|l|}{ Outros } \\
\hline FoE_Naka 3.4.1 & L: $(1,2,3,4,5,8)$; & 6 \\
\hline DsFwaveltc 3.4 .2 & ITL: [1:1:20]; & 20 \\
\hline LO Smoothing 3.4 .3 & ITL: [1:1:10]; Lambda: [0.005: 0.005: 0.1]; Kappa: [1.05:0.35: 9.8]; & 5200 \\
\hline BLF_LO 3.4.4 & $\begin{array}{l}\text { ITL: [1:1:3]; Sigma S: 1; Sigma R: [5:40:45]; Lambda: [0.01: 0.01:0.1]; } \\
\text { Kappa: [1:4:9] }\end{array}$ & 180 \\
\hline PPB 3.4 .5 & $\begin{array}{l}\text { ITL: [1:1:5]; Number of Looks: [1:2:5]; Half Search Window Size: [8:2:12]; } \\
\text { Half Sliding Window Width: [3:2:7]; Alpha: [0.01: 0.3:1]; T: [0.01: 0.3:1]; }\end{array}$ & 2160 \\
\hline SARBM3D 3.4.6 & ITL: [1:1:20]; SWS: [3:2:19]; & 180 \\
\hline
\end{tabular}

Conforme visto no capítulo 5, cada filtro avaliado neste estudo teve seus

Tabela 6.1: Intervalo de variação de cada parâmetro para cada filtro e o total de variações usadas para cada filtro. ITL (Iteration Length) representa quantas vezes um filtro foi reaplicado, enquant SWS (Sliding Window Size) o tamanho da janela deslizante utilizada.

parâmetros variados para que diferentes configurações pudessem ser testadas. O 
intervalo de variação de cada parâmetro foi definido primeiro de forma empírica e depois minimizado de forma não rigorosa em testes preliminares com a análise de gráficos apresentada na seção 5.1, apesar de alguns autores sugerirem limites superiores e inferiores para cada parâmetro de seus filtros que foram também respeitados.

A Tabela 6.1 contém todas as variações de parâmetros, como vistos na notação presente na seção anterior, com a exceção do FoE_Naka que possui 6 valores pré-definidos pelos autores e do terceiro parâmetro do DsFsrad, a Região de Interesse (ROI), que foi fixada empiricamente. A última coluna, Total, representa o total de variações feitas em um mesmo filtro para uma única imagem de teste.

Vale ressaltar que apesar do filtro DSDN possuir 4 parâmetros, este cria 5 imagens diferentes para cada configuração de parâmetros, cada uma relativa a quantidade de reaplicações do filtro, realizados internamente, com a primeira imagem tendo sido a melhor segundo as avaliações feitas.

Somando-se todas as variações mencionadas na Tabela 6.1, 15.322 imagens resultantes foram geradas para cada imagem de teste e, como dito na seção 5.1, 18 casos de teste diferentes foram testados, totalizando assim 275.796 imagens.

Os 18 casos de teste usados são fatias 2D, dos planos sagital, coronal e transversal, de um exame de ultrassom 3D volumétrico já em coordenadas cartesianas no Modo B.

\subsection{Escolha do total de Clusters}

Originalmente haviam-se adotado ambos os métodos de escolha do total de clusters no $k$-means, entretanto notou-se que os casos que usavam o kSqrt frequentemente criaram imagens com qualidade visual superior a dos casos que usaram o Critério de Gap, indicando que o uso somente do kSqrt já poderia ser suficiente.

Conforme visto na Figura 6.1, o método empírico gera resultados quase idênticos ou ligeiramente mais homogêneos que o Critério de Gap (comparando as imagens $\mathrm{AxB}, \mathrm{CxD}, \mathrm{ExF}, \mathrm{GxH}, \mathrm{IxJ}$ ). Constatou-se assim que apesar do Critério de Gap fornecer um total de clusters que melhor se assemelha aos dados apresentados, frequentemente esse valor é maior que o obtido com o kSqrt, obtendo assim mais grupos e consequentemente grupos com menos elementos.

Com grupos menores, um possível caso espúrio e pouco representativo gerado durante os testes acaba sendo mais relevante e ao ser agrupado, pode alterar significativamente o centroide do seu cluster e assim comprometendo a avaliação.

Ao gerar uma quantidade total de clusters menor e consequentemente grupos com um número maior de elementos, o kSqrt acaba diminuindo a relevância dos casos espúrios, garantindo assim um centroide mais confiável. Baseado nessa análise, somente o kSqrt será usado no restante deste trabalho.

\subsection{Cardinalidade Relativa x Performance}

Aplicando a análise dos parâmetros obtidos pelos centroides dos clusters de Cardinalidade Relativa e de Performance, as Figura 6.2 e a Figura 6.3 foram geradas. A quarta e quinta imagem de cada figura representam as imagens originais de entrada e o ground-truth, respectivamente, enquanto as outras imagens 
representam o melhor resultado obtido pelo agrupamento por $k$-means em cada métrica de agrupamento.

Nesta comparação visual é possível observar como os resultados do cluster de maior Cardinalidade Relativa (Figura 6.2) se comportam melhor que o cluster de maior Performance (imagens equivalentes na Figura 6.3), onde o primeiro apresenta imagens mais nítidas, com fronteiras melhor definidas enquanto ainda mantém as principais características da imagem, ao passo que a segunda figura gerou imagens borradas e com bordas deformadas em relação à imagem original. Esse padrão se repetiu ao longo de todos os filtros testatos e, portanto, somente a avaliação de Cardinalidade Relativa será usada nas próximas avaliações.

\subsection{Clusterização x Análise por Gráfico}

Comparando ainda a avaliação feita pelo método de agrupamento por $k$ means (Figura 6.2 e Figura 6.3) com as avaliações por gráfico (Figura 6.4), observamos como o primeiro é mais conciso e objetivo, apresentando um único resultado que pode ser facilmente testado, enquanto o segundo é propenso a erros de visualização e escala, visto que casos como o apresentado na Figura 6.4 foram recorrentes. Nesta última figura não é possível dizer facilmente quais seriam os candidatos a melhores parâmetros uma vez que não existe uma convergência clara ou significativa em nenhum dos parâmetros observados.

A Figura 6.4 apresenta dois gráficos, ambos do filtro SADCTD e métrica de agrupamento FR, o superior para o parâmetro P1, intitulado "AllFRDsP1Merged" e o inferior para o parâmetro P2, intitulado "AllFRDsP2Merged". Os 80 resultados gerados por este filtro para cada um dos 18 casos de teste são apresentados, totalizando 1.440 elementos em ambos os gráficos.

No gráfico "AllFRDsP1Merged", existe uma maior concentração de elementos com a pontuação no intervalo [0.24, 0.26], entretanto isso acontece tanto para P1 com valor 1 quanto para P1 com valor 2, tornando esta análise inconclusiva.

$\mathrm{O}$ fato apresentado anteriormente também acontece no segundo parâmetro, visto no gráfico AllFRDsP2Merged, sendo a análise aqui ainda mais difícil por não existir nenhuma concentração relevante e mesmo que a seleção do elemento com maior pontuação fosse feita, não existe garantia que este elemento não seja um outlier, uma vez que aparentemente somente 1 outro elemento, dentre os 1440 resultantes deste filtro, está próximo, no gráfico, daquele de maior pontuação.

\subsection{Tipos de Pontuação}

A Figura 6.5 contém o resultado da avaliação do filtro PPB_Nakagami com quatro imagens de entrada diferentes, onde cada coluna da figura representa o melhor resultado obtido por uma mesma métrica de agrupamento, segundo a análise feita por $k$-means em modo de Cardinalidade Relativa e definição de $k$ por kSqrt, enquanto a primeira e a segunda coluna contém as imagens originais e ground truth usadas para a geração dos testes.

Relembrando o que foi mencionado na seção 4.3, as métricas de agrupamento utilizadas na Figura 6.5 são as da categoria Full Reference (FR), Reduced Reference (RR) e No Reference (NR), todas utilizando o sistema de peso Light e Weighted, com a exceção da categoria NR, que por possuir uma única métrica seria redundante utilizar o sistema de pesos, sendo, portanto simplificado para apenas NR Light, totalizando assim 7 avaliações distintas. 
Nesta figura, as imagens nas colunas de RR, Light e Weighted não apresentam resultados satisfatórios, na coluna NR as imagens foram degradadas, ao passo que as imagens da coluna FR Light apresentam consistentemente imagens que seguem as diretivas apresentadas no Capítulo 2.

Os resultados foram inadmissíveis para o RR Weighted em $50 \%$ dos 18 casos de testes utilizados, em $83 \%$ no RR Light e em $33 \%$ no NR ao passo que as outras métricas de agrupamento não apresentaram resultados ruins em nenhum dos testes realizados.

Apesar das métricas discutidas a seguir apresentarem uma alta incidência de resultados que obtiveram boa pontuação ao deformarem drasticamente a imagem de entrada, os seguintes casos conseguiram atingir os requisitos propostos no capítulo 3: Os filtros SADCTD, FoE_Naka, L0, DSDN, BLF_L0 e Bilateral para a métrica NR, DSDN e SARBM3D para a métrica RR Weighted e FoE_Naka e DSDN para a métrica RR Light. O Anexo A está disponível para apresentar a aplicação de todos os filtros em todos os casos de teste segundo critérios que serão detalhados na seção 6.8 .

Já a avaliação das métricas agrupadas por Light e Weighted nos levou a conclusão de que apesar dos pesos terem sido arbitrados após análise da qualidade visual das imagens, eles foram insuficientes para que resultados melhores que os da classificação Light fossem gerados. Em todas as quatro categorias apresentadas, All, FR, RR e NR, a avaliação por Light foi responsável por garantir imagens que melhor seguiam os Requisitos Fundamentais e Características Desejáveis vistos anteriormente.

\subsection{Clusterização sem Métricas de Agrupamento}

Visto que a atribuição de pesos nas métricas não contribuiu para um melhor resultado, um outro tipo de avaliação foi possível, utilizando o resultado das métricas em si na clusterização ao invés das métricas de agrupamento e testando novamente para as categorias All, FR, RR e NR, ou seja, quatro novos clusters foram criados para cada filtro, o primeiro utilizando todas as métricas apresentadas, o segundo com somente as métricas Full Reference, o terceiro apenas com as métricas de Reduced Reference e o último só com a métrica MetricQ.

Observando ainda que a escolha de centróide por melhor Perfomance também não gerou bons resultados, aqui somente a Cardinalidade Relativa foi levada em consideração.

Este teste entretanto também foi insuficiente em gerar resultados consistentes, com somente $20 \%$ dos casos analisados satisfazendo os Requisitos Fundamentais e Características Desejáveis e ainda assim com imagens visualmente piores em relação aos testes com as métricas de agrupamento.

Vale ressaltar que novamente a categoria Full Reference obteve o melhor resultado dentre os quatro tipos de métricas abordados.

Pelos fatos observados anteriormente, o uso das métricas de agrupamento continuará nas próximas avaliações. 


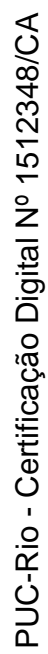

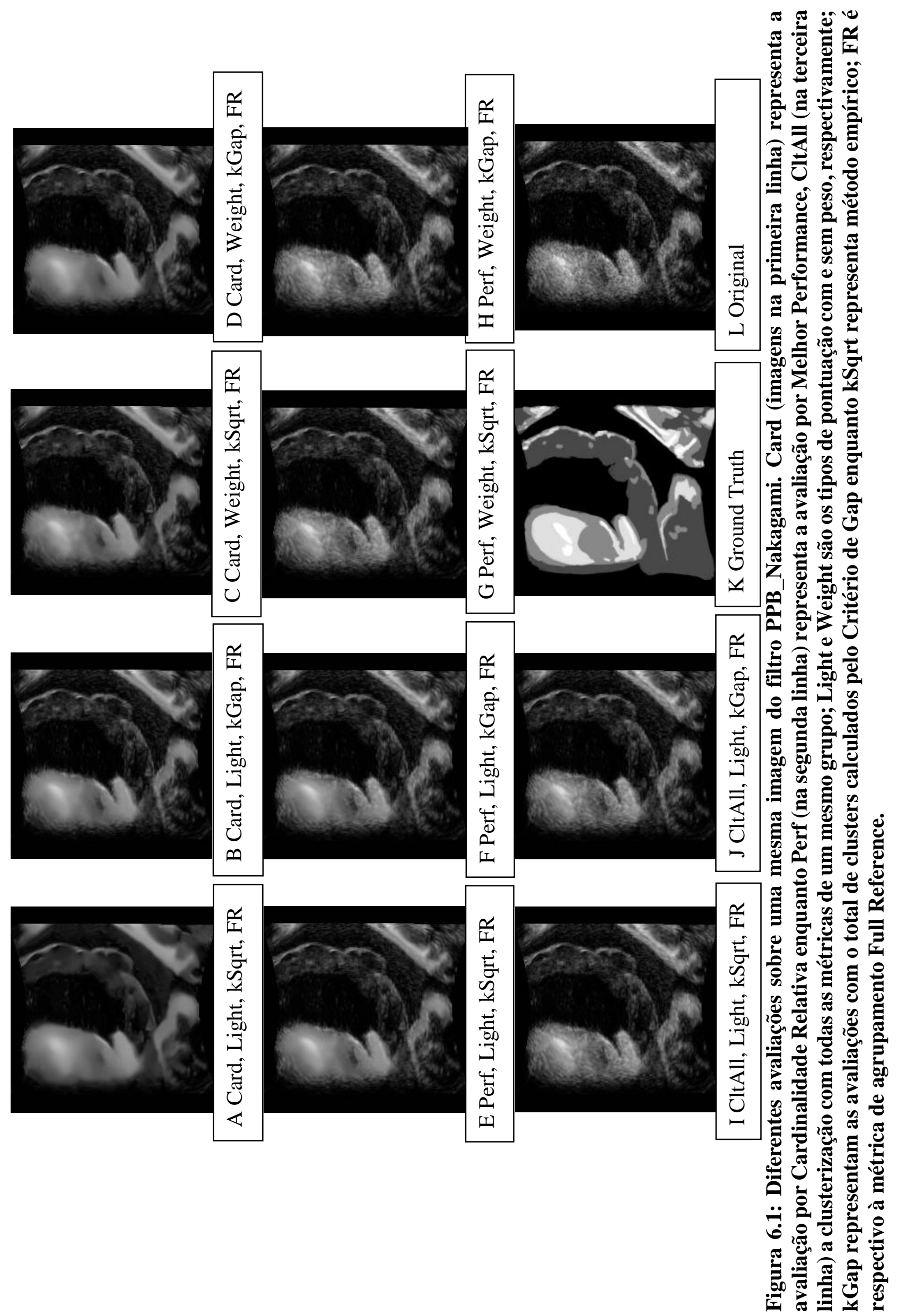



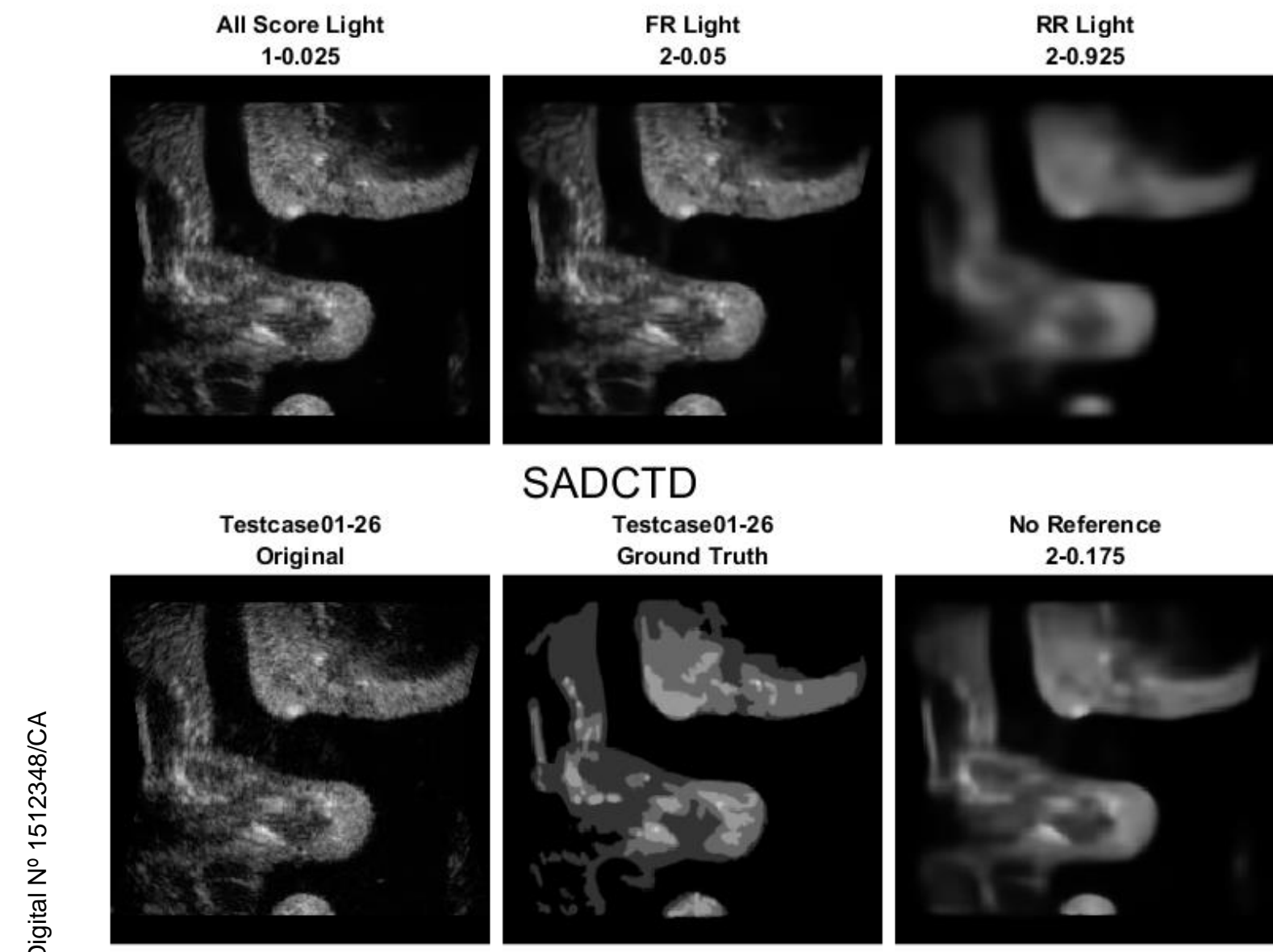

\section{SADCTD}

Testcase 01-26

Ground Truth

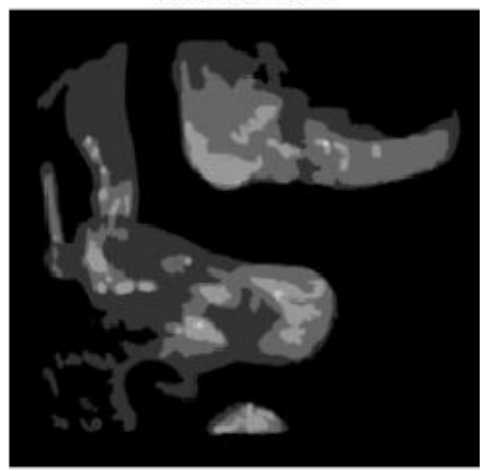

No Reference 2-0.175

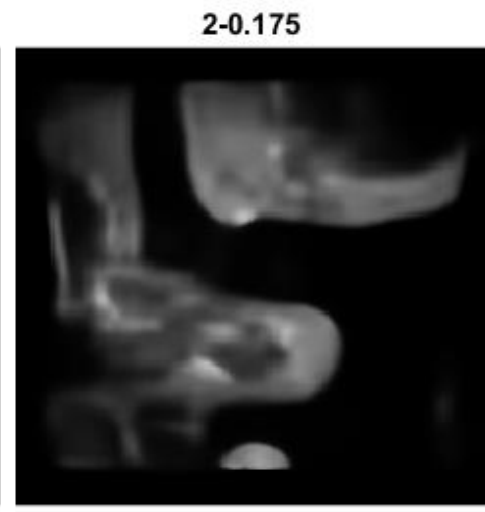

All Score Weighted 2-0.7

FR Weighted 2-0.875

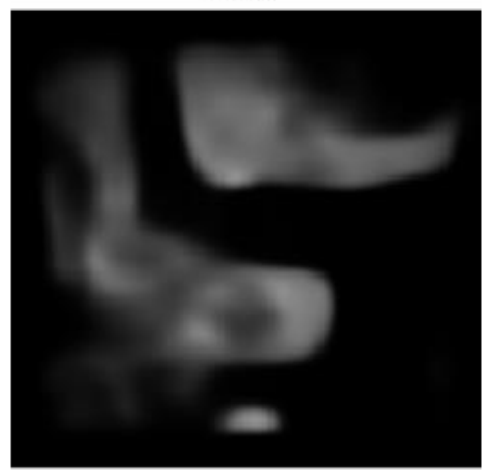

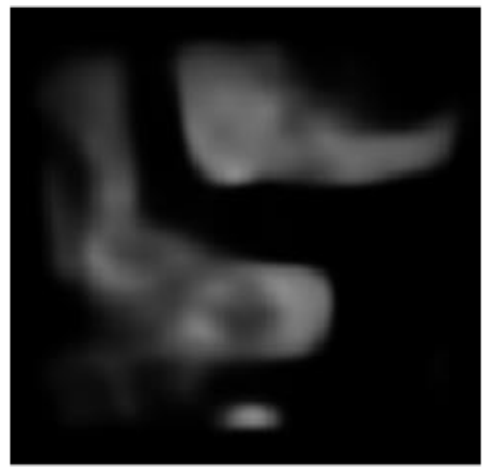

RR Weighted

2-0.05

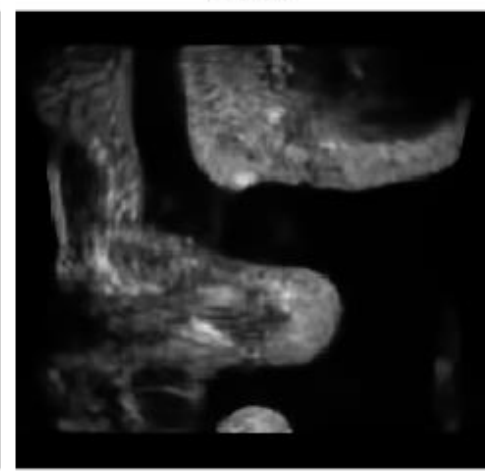

Figura 6.2: Avaliação da aplicação dos parâmetros encontrados pelo centroide de maior Cardinalidade Relativa ao filtro SADCTD. Os números do título de cada imagem representam os parâmetros usados no filtro, como apresentados no capítulo 3, com exceção das imagens Original e Ground Truth. 

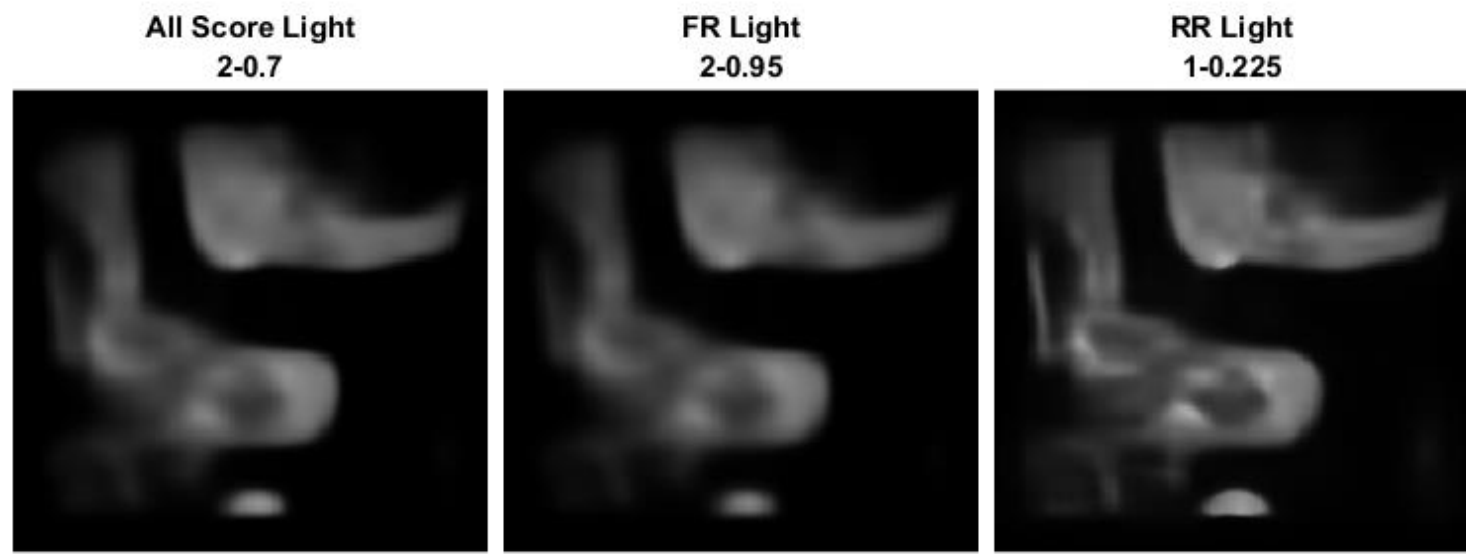

\section{SADCTD}
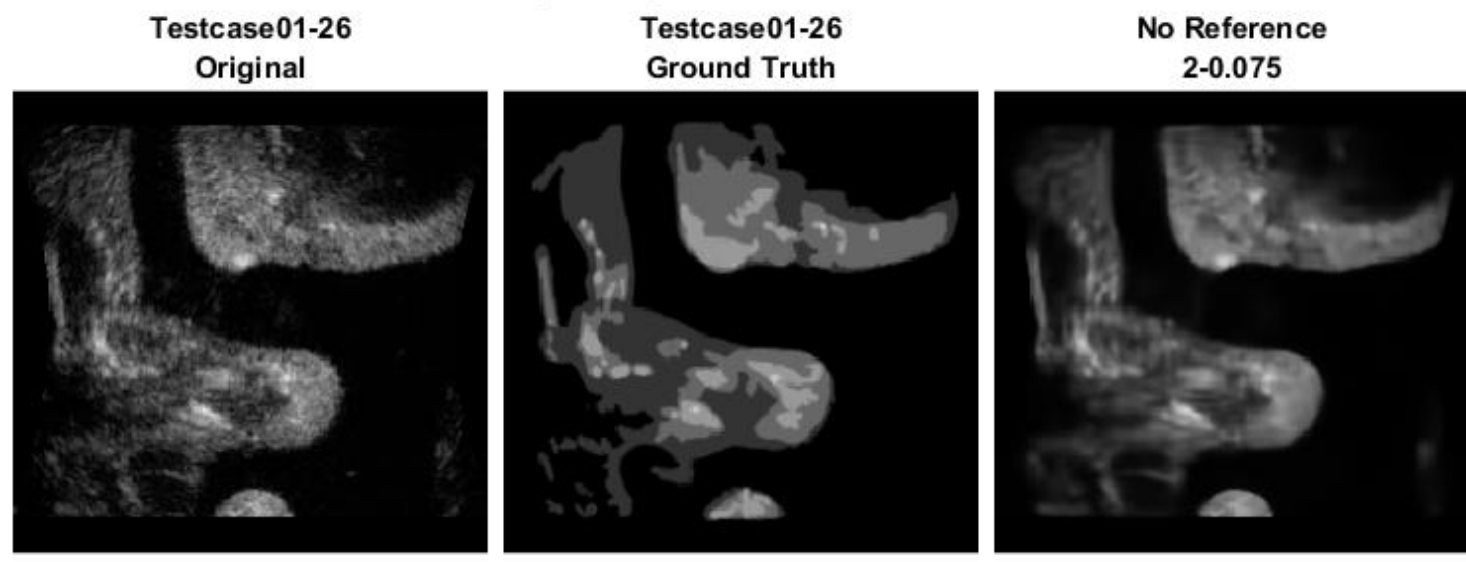

All Score Weighted 2-0.225

FR Weighted

$2-0.95$

RR Weighted

$2-0.95$
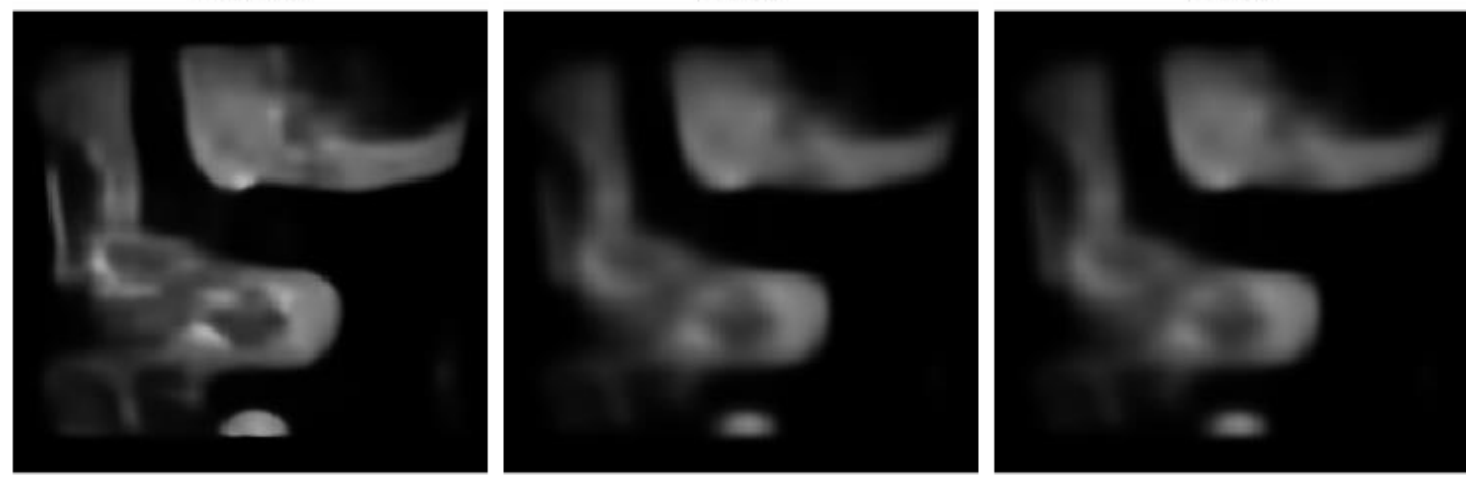

Figura 6.3: Avaliação da aplicação dos parâmetros encontrados pelo centroide de maior Performance ao filtro SADCTD. Os números do título de cada imagem representam os parâmetros usados no filtro, como apresentados no capítulo 3, com exceção das imagens Original e Ground Truth. 


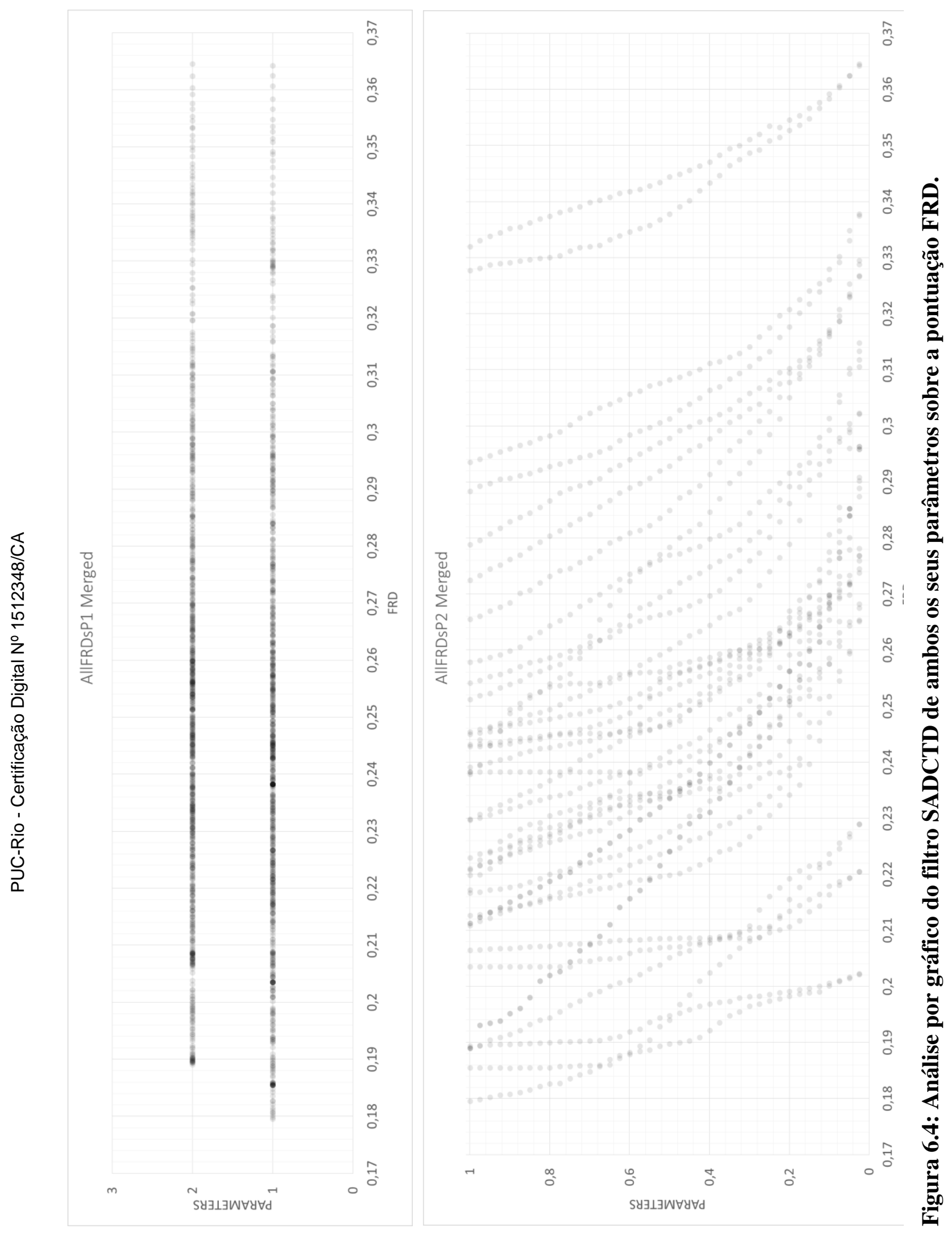



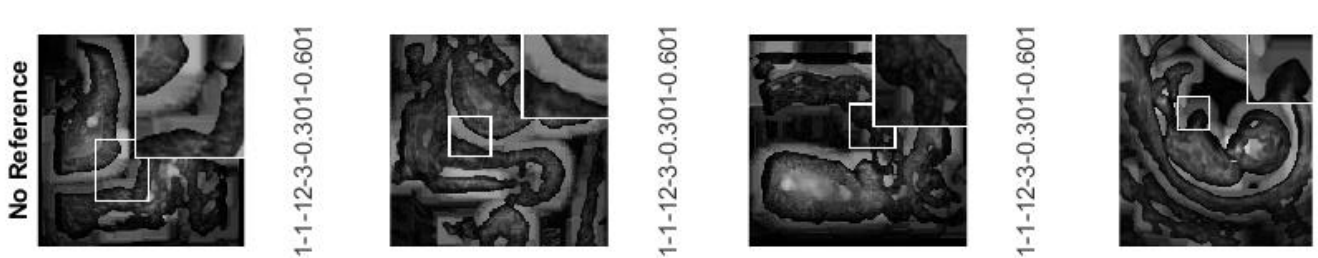

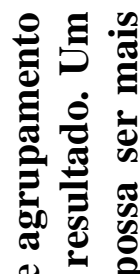
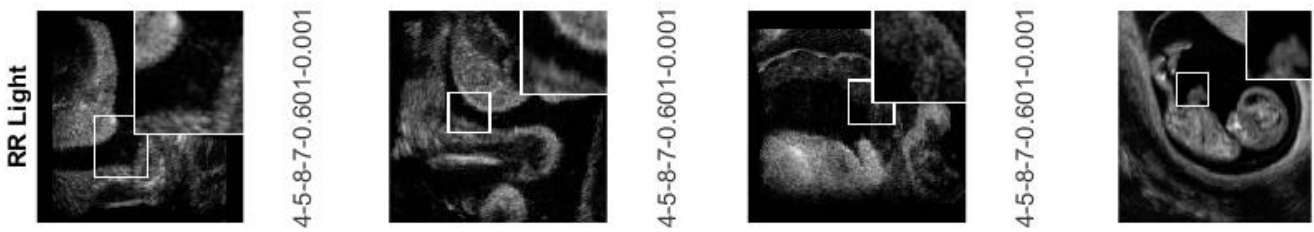

응

起 율

불

'巫

$\wedge$ ํํㅇ

๘ ฮี 즐
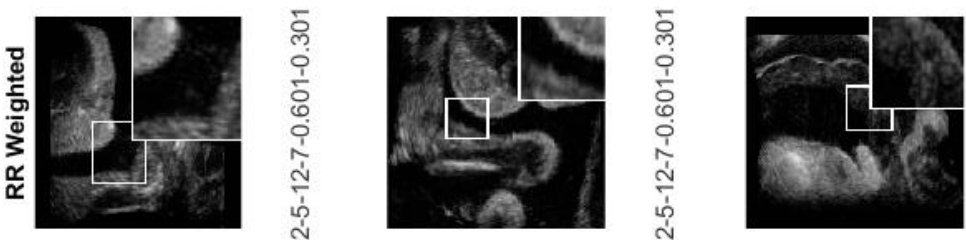

을 클

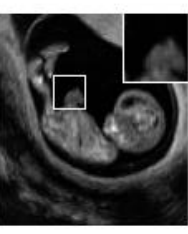

อ

ซ ฟै

:

을 을
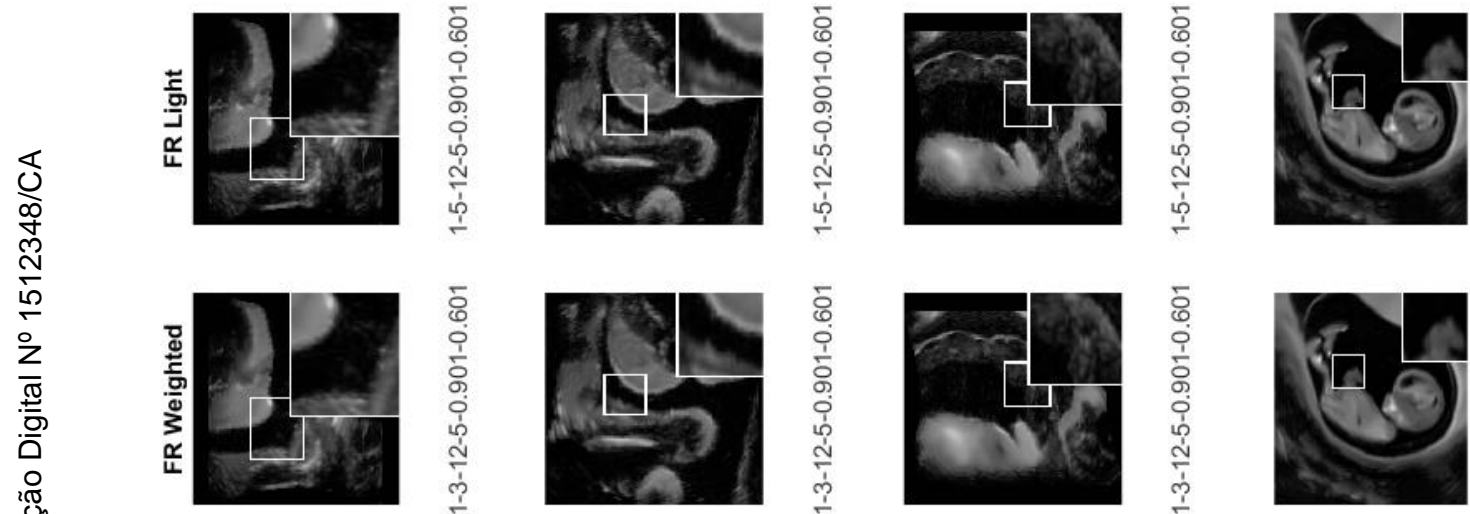

을 응
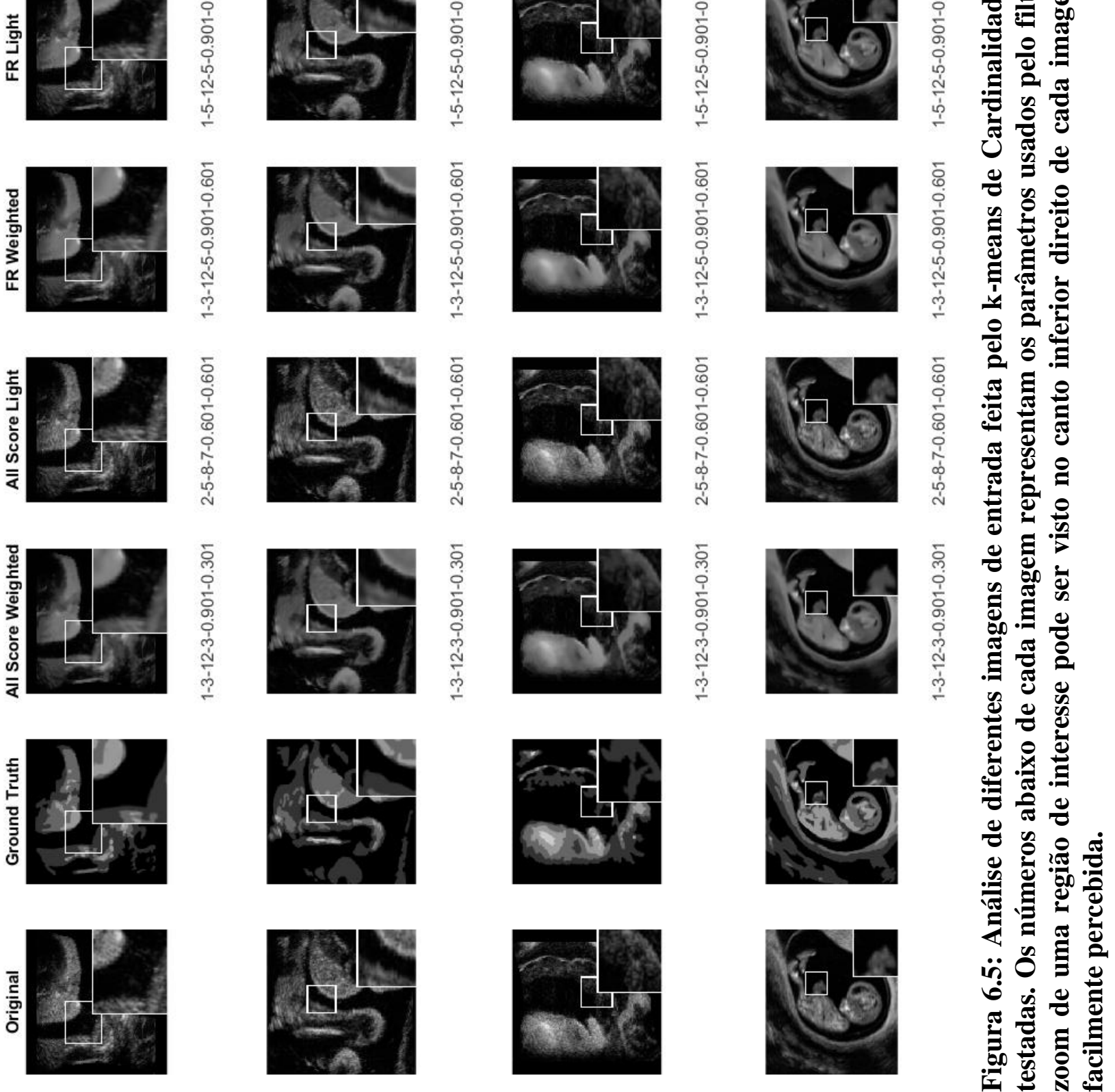


\subsection{Análise normalizada das métricas}

Optou-se ainda por avaliar a performance dos filtros de acordo com o somatório dos melhores resultados obtidos em cada imagem de teste e normalizálos para o intervalo $[0,1]$, como visto na Tabela 6.3. Esta avaliação também fez uso das categorias Light e Weighted, que resultou na Tabela 6.4.

Para facilitar a leitura das tabelas citadas anteriormente, encontram-se em negrito os três melhores resultados em cada métrica de agrupamento.

Esta avaliação nos permite uma melhor compreensão do comportamento que cada filtro tem perante cada métrica, como por exemplo o filtro SADCTD que teve uma performance ruim nas métricas MSE, SNR, M3, M4, entre outras, ao passo que teve quase o melhor resultado na métrica SMPI. Uma interpretação para isso é que este filtro tende a alterar severamente a imagem de entrada enquanto ainda se afasta da imagem ground truth gerada pelo especialista, suavizando de uma forma diferente da esperada e não criando fronteiras tão acentuadas quanto o ground truth. Entretanto este filtro consegue manter características importantes da imagem original e remover eficientemente o ruído speckle, garantindo assim uma boa pontuação para o SMPI.

Outro exemplo a ser observado é o caso do Bilateral Filter (shiftable_BF), que obteve um bom desempenho em diversas métricas e atingindo em especial o melhor resultado na Field of Metrics (FOM), o que significa que este filtro possui boas características para preservação de arestas. Entretanto ele obteve um resultado ruim na métrica ESI, que pontua de acordo com a preservação de aresta em relação a imagem ruidosa, significando que este também é um filtro que modifica drasticamente uma imagem, apesar de neste caso a imagem modificada se aproximar mais do ground truth apresentado.

Com a aplicação das métricas de agrupamento, foi possível observar mais facilmente o comportamento geral dos filtros e o intuito dessa avaliação era poder ao menos eliminar alguns filtros da comparação final, entretanto notou-se que esses resultados não eram condizentes com a avaliação visual observada. O SADCTD esteve entre os 5 piores resultados em todas as métricas de agrupamento apesar do mesmo ter gerado consistentemente uma das melhores avaliações visuais. O DsFlsminsc esteve sempre a menos de $10 \%$ de diferença das melhores performances obtidas, entretanto suas imagens resultantes falharam em remover/suavizar o ruído speckle ou gerar áreas mais homogêneas.

A conclusão é que o método de avaliação apresentados na Tabela 6.3 e Tabela 6.4 , ao pontuar os melhores resultados para cada métrica, acaba muitas vezes por selecionar outliers e assim priorizar filtros que possuam muitas variações de parâmetros, uma vez que a probabilidade de um dos seus possíveis resultados de favorecer uma das métricas é maior do que com filtros que possuam poucas variações.

\subsection{Discussão}

Como já havia sido mencionado na seção 4.1.1, as métricas mais utilizadas na literatura encontram-se na categoria Full Reference, sendo assim necessária uma imagem de ground truth para atribuir uma pontuação à imagem filtrada ao comparála com uma imagem ideal.

Visto também que um especialista em tratamento manual e semiautomático de exames de ultrassom para reconstrução e impressão 3D foi responsável por criar 
as imagens ground truth, estas estão sujeitas ao seu julgamento, não sendo, portanto, perfeitas, podendo diferir das estruturas reais encontradas no exame.

Pelos motivos apresentados anteriormente, não é possível afirmar que uma configuração de filtro que obteve uma pontuação em uma determinada métrica ou conjunto de métricas de fato cumpre com os objetivos propostos no capítulo 2 , sendo então necessário uma última avaliação para confirmar se uma boa pontuação em uma configuração de filtro realmente gerou uma imagem com áreas mais homogêneas e manteve as fronteiras originais do exame: a avaliação visual.

O intuito da avaliação visual é comprovar que uma boa pontuação em uma ou mais métricas podem ser correlacionadas com os objetivos propostos, podendo inclusive, em casos como nas métricas Reduced Reference ou No Reference, servirem para filtragens não supervisionadas, onde a imagem de interesse é refinada por essas métricas até que um patamar seja atingido.

Em outras palavras, as avaliações propostas até aqui tinham como objetivo testar as configurações possíveis de cada filtro e procurar por alguma maneira de averiguar se era possível detectar automaticamente aquelas configurações e aqueles filtros que preservassem as principais características dos exames enquanto ainda removessem o ruído speckle e suavizassem regiões de não-fronteiras. Dentro do escopo deste estudo não foram encontradas outras maneiras de realizar esta averiguação que não confrontando os dados das métricas com uma avaliação visual.

Diversos levantamentos foram realizados acerca de como melhor avaliar os resultados dos filtros e ao longo do capítulo 6 foi possível observar como alguns dos métodos abordados eram menos robustos que outros, acarretando em seu descarte na avaliação final.

Finalmente, baseado no que foi apresentado, entendemos que os métodos de avaliação mais apropriados para medir a qualidade de filtros speckle são a clusterização por k-means; com seleção dos centroides por Cardinalidade Relativa; total de clusters calculado por kSqrt; na categoria Light; e agrupamento por Full Reference.

\subsection{Parâmetros mais apropriados}

Baseado nas conclusões anteriores, os parâmetros de cada filtro foram determinados de acordo com o método de seleção de centroide dos clusters por Cardinalidade Relativa, com o número total de clusters definido por kSqrt, na métrica de agrupamento Full Reference, em que todas as métricas deste grupo ficaram com o mesmo peso, sendo assim na categoria Light, com suas configurações apresentados na Tabela 6.2 a aplicação dessas configurações vistos na Figura 6.6.

Esta figura contém no canto inferior direito de cada imagem um zoom de uma região de interesse em que se é possível ver com melhor clareza o efeito de cada filtro.

Apesar das três equações apresentadas para o DsFad, mencionados na seção 3.3.1, este é considerado como um único filtro, com cada equação sendo resultado da variação de um parâmetro.

O filtro FoE_Naka, por outro lado, possui um único parâmetro pois os autores desenvolveram conjuntos de configurações de parâmetros e os disponibilizaram junto com a implementação do filtro. $O$ valor passado para este filtro é responsável por selecionar um dentre os 6 conjuntos de configurações disponíveis. 
No geral as avaliações feitas resultaram em parâmetros "conservadores", com seus valores concentrados próximo ao limite inferior de seus domínios. Apesar disso, alguns filtros tiveram valores mais próximos ao limite superior, como o número de iterações do DsFhomo, o Half Search Window Size do PPB_Nakagami, entre outros.

Com base no que foi apresentado, os parâmetros determinados para os filtros Bilateral, L0 e BLF_L0 resultam em aplicações que frequentemente criam regiões homogêneas, apesar de em alguns casos isso ter se mostrado excessivo, ocasionando em uma perda de determinadas estruturas.

\begin{tabular}{|c|c|}
\hline Filtros & Parâmetros \\
\hline \multicolumn{2}{|l|}{ Lineares } \\
\hline DsFlsmv 3.1.1.1 & ITL: 2; SWS: 3; \\
\hline DsFwiener2 3.1.1.2 & ITL: 3; SWS: 3; \\
\hline DsFlsminsc 3.1 .2 & ITL: 2; SWS: 5; Edge Detector: 3; \\
\hline \multicolumn{2}{|l|}{ Não Lineares } \\
\hline DsFmedian 3.2.1 & ITL: 4; SWS: 7; \\
\hline DsFca 3.2 .2 & ITL: 3; SWS: 2; \\
\hline DsFls 3.2 .3 & ITL: 2; SWS: 3; \\
\hline DsFlecasort 3.2.4 & ITL: 2; SWS: 3; \\
\hline DsFhomog 3.2.5 & ITL: 3; SWS: 4; \\
\hline DsFgf4d 3.2.6 & ITL: 2; SWS: 11; \\
\hline DsFhomo 3.2 .7 & ITL: 14; SWS: 3; \\
\hline DSDN 3.2.8 & Noise Type: 3; Fusing: 1; Window Type: 2; Symmetry Type: 1; \\
\hline SADCTD 3.2 .9 & Sigma Limit: 2; Sigma Delta: 0.05; \\
\hline \multicolumn{2}{|l|}{ DIFUSÃO } \\
\hline DsFad 3.3.1 & ITL: 1; Kappa: 11; Lambda: 0.05; Option: 1; \\
\hline DsFsrad 3.3.2 & ITL: 10; Lambda: 0.71; ROI; \\
\hline DsFlndif 3.3 .3 & ITL: 1; Lambda: 5; Sigma: 1.81; Difusivity Speed: 4; Step Size: 1; \\
\hline Bilateral 3.3.4 & ITL: 1; Sigma S: 3; Sigma R: 29; \\
\hline Trilateral 3.3.5 & ITL: 2; Sigma C: 1; Epsilon: 0.08; Beta: 0.9; \\
\hline \multicolumn{2}{|l|}{ Outros } \\
\hline FoE_Naka 3.4.1 & L: 8; \\
\hline DsFwaveltc 3.4 .2 & ITL: 1; \\
\hline LO Smoothing 3.4 .3 & ITL: 1; Lambda: 0.025; Kappa: 8.4; \\
\hline BLF_LO 3.4.4 & ITL: 1; Sigma S: 1; Sigma R: 5; Lambda: 0.03; Kappa: 9; \\
\hline $\begin{array}{l}\text { PPB_Nakagami } \\
3.4 .5\end{array}$ & $\begin{array}{l}\text { ITL: 1; Number of Looks: 5; Half Search Window Size: 12; Half Sliding Window } \\
\text { Width: 5; Alpha: 0.901; T: 0.601; }\end{array}$ \\
\hline SARBM3D 3.4 .6 & ITL: 4; SWS: 5; \\
\hline
\end{tabular}

Tabela 6.2: Valores definidos dos parâmetros de cada filtro de acordo com o método de seleção de centroide dos clusters por Cardinalidade Relativa, com o número total de clusters definido por kSqrt, na métrica de agrupamento Full Reference, na categoria Light.

\subsection{Filtros}

Somando todas as configurações testadas para todos os filtros apresentados e em todos os casos de teste, 275.796 imagens foram obtidas e após as conclusões obtidas nas últimas seções, 378 imagens foram avaliadas como as melhores segundo as conclusões obtidas até então, um para cada caso de teste e para cada filtro, utilizando as configurações apresentadas na Tabela 6.2.

Os filtros DsFad, DsFca, DsFgf4d, DsFlecasort, DsFlsminsc, DsFnldif, DsFsrad, DsFwaveltc e DsWiener falharam em seguir as diretivas apresentadas no 
Capítulo 2, não obtendo resultados consistentes ou até mesmo deformando ou degradando as imagens de teste.

Já os filtros BLF_L0, DSDN, L0, FoE_Naka, SARBM3D, PPB_Nakagami e SADCTD tiveram um desempenho ótimo, com destaque para os três últimos, com o PPB_Nakagami criando regiões homogêneas mesmo em estruturas mais complexas, enquanto ainda preservando as fronteiras e as principais características visuais dos exames, o SADCTD eficientemente removendo o ruído speckle e o SARBM3D apresentando bons resultados em todos os pontos observados.

PPB_Nakagami, SADCTD e SARBM3D muitas vezes apresentaram resultados visuais melhores que o próprio ground truth desenvolvido pelo especialista ao criarem transições mais suaves nos tons de cinza entre áreas vizinhas e respeitarem mais as fronteiras existentes na imagem ruidosa.

Tanto o BLF_L0 quanto L0 também conseguiram um bom desempenho, entretanto sua aplicação não supervisionada não é recomendada uma vez que ambos foram responsáveis por suavizar demais algumas áreas das imagens de teste, frequentemente perdendo informação sobre algumas regiões existentes, como visto na Figura 6.6.

Os filtros restantes, apesar de não terem degradado as imagens de teste, também não foram capazes de criar resultados satisfatórios segundo as diretivas apresentadas e discutidas extensamente ao longo deste trabalho, não sendo assim bons candidatos para este trabalho.

Uma outra forma de visualizar o desempenho dos filtros aqui utilizados é apresentada no 
Anexo B, em que a escala de cores adotada para esta visualização apresenta um tom de amarelo quando a mediana obteve pixels mais claros que a imagem ground truth, uma cor preta quando os pixels de ambas as imagens possuíram intensidades semelhantes e um tom magenta quando a imagem ground truth ficou com pixels mais claros que a outra imagem. Todas as imagens deste anexo foram realçadas com um acréscimo de brilho e contraste para que as diferenças pudessem ficar mais evidentes.

Esta visualização nos permite observar como, na maioria dos casos, as áreas internas das estruturas presentes nos exames de ultrassom foram menos homogeinizadas em relação ao ground truth, com mais informações preservadas que o próprio trabalho manual do especialista que gerou a imagem de ground truth em si. Por outro lado, o ruído presente no líquido amniótico foi menos suprimido pelos filtros que pelo trabalho do especialista, indicado pela maior incidência de áreas amareladas nas imagens.

As imagens do 
Anexo B também exibem, em alguns casos, um tom magenta claro, em geral presente em algumas fronteiras, indicando que o especialista suavizou demais algumas áreas, perdendo assim informações das fronteiras entre as estruturas contidas no exame e o líquido amniótico.

\subsection{Métricas}

Uma vez que tanto os filtros quanto as configurações mais apropriadas para resolver o ruído speckle foram apresentados nas seções anteriores, foi possível observar individualmente o comportamento das métricas para enfim determinar quais são mais adequadas para indicar automaticamente que uma imagem segue as diretivas apresentadas no capítulo 2 .

As Tabela 6.5, Tabela 6.6 e Tabela 6.7 contém os resultados de todas as métricas, normalizadas no intervalo [0,1], para os filtros PPB_Nakagami, SADCTD e SARBM3D, respectivamente, em todos os casos de teste. Além disso, nas últimas linhas encontram-se a média, mediana e o melhor resultado dentre todos os casos de teste. Em negrito estão os 3 melhores resultados de cada métrica ao longo do conjunto de testes, enquanto em itálico e sublinhado estão os resultados de média e mediana com valores acima de 0.8 , que foi definido empiricamente para auxiliar a encontrar as melhores métricas.

Nas três tabelas, as métricas MSE, SNR, AD, SC, NK, COC e Beta obtiveram resultados próximos a 1, com exceção da Tabela 6.7 (SARBM3D), que apresentou um Beta com média 0.77 e mediana 0.75 . Esses valores corroboram com o fato de que estas métricas consistentemente pontuam bem as imagens que exibem regiões mais homogêneas além de preservar as fronteiras dos exames originais e remover o ruído speckle.

Apesar de não terem atingido o patamar de 0.8 nos três filtros, as métricas CQ e CTQ tiveram médias 0.88 e 0.89 para o PPB_Nakagami, 0.76 e 0.74 para o SADCTD e 0.79 e 0.78 para o SARBM3D, podendo também serem consideradas adequadas para a avaliação de desempenho de filtros para tratamento de imagens de ultrassom.

É importante notar que apesar da métrica UQI ser popular na literatura, para os casos de teste apresentados, ela obteve médias abaixo de 0.5 nos três filtros, possivelmente indicando que esta métrica é fortemente prejudicada pela baixa qualidade visual dos exames de ultrassom.

O valor desprezível obtido na métrica ESI (Reduced Reference) indica que pode ter ocorrido um equívoco ao determinar seu limite superior teórico, uma vez que todos os seus valores foram arredondados para 0.01 ou que esta métrica de fato não é adequada para os testes aqui realizados ou ainda que foi dúbia a adoção da diagonal para unir sua avaliação horizontal e vertical em uma única métrica.

A MetricQ, única disponível na categoria No Reference e a métrica SSI, da categoria Reduced Reference, também obtiveram baixos resultados, com médias na ordem de 0.2 e 0.03 , respectivamente, nos levando a acreditar que as mesmas observações feitas a respeito da ESI podem ser feitas nestes casos.

O restante das métricas, M3, M4, SSIM, MD, LMSE, NAE, FOM e SMPI, ficaram com resultados contidos no intervalo [0.3, 0.7], com suas médias possuindo baixa variabilidade entre os três filtros apresentados. Concluímos que essas 8 métricas não são adequadas para avaliar o desempenho dos filtros segundo as diretivas apresentadas no capítulo 2. 


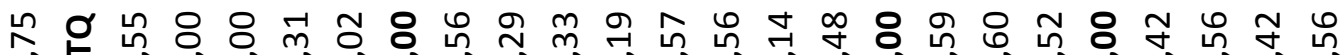

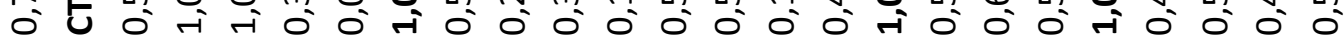

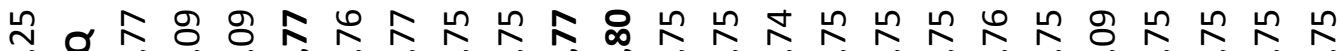
o $\begin{aligned} & 0 \\ & 0\end{aligned}$

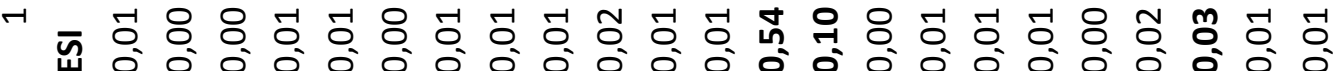
$\infty$ ㅁ 8 ○

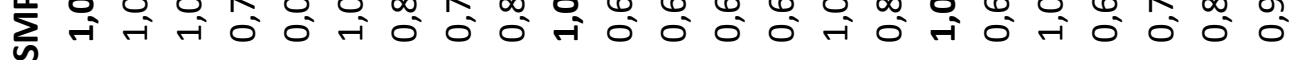
ஸ̃

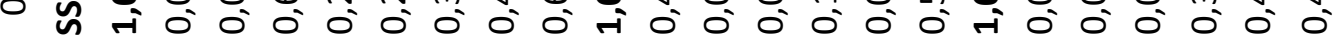

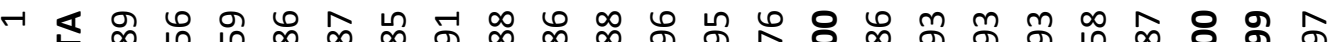

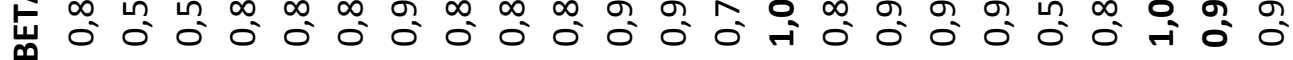
$\rightarrow$ 욱 8 욕

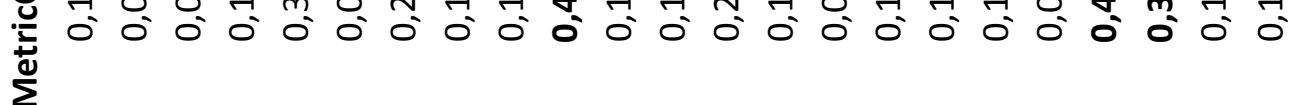

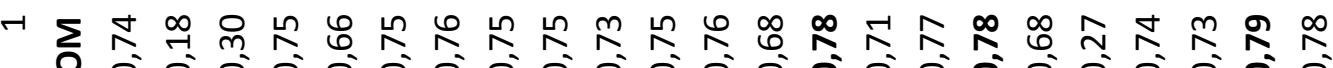

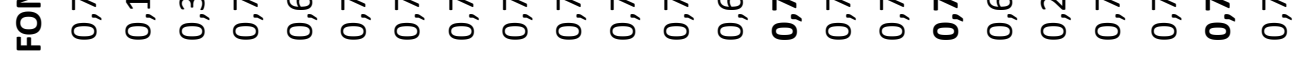

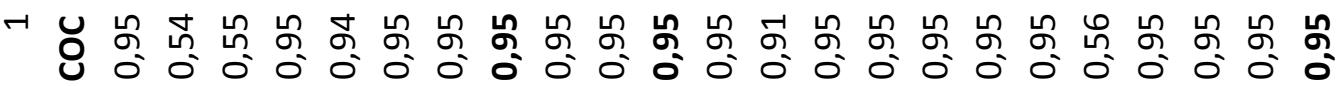
㫕

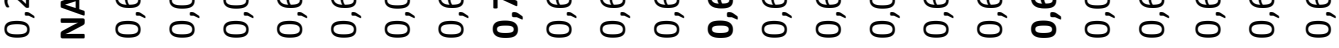

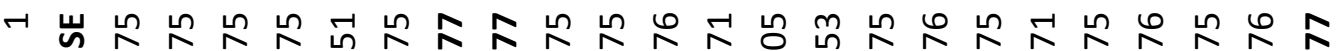

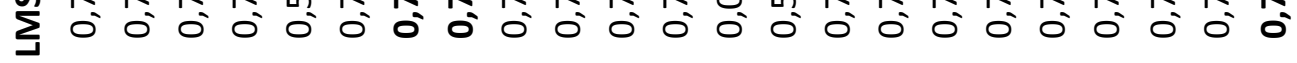

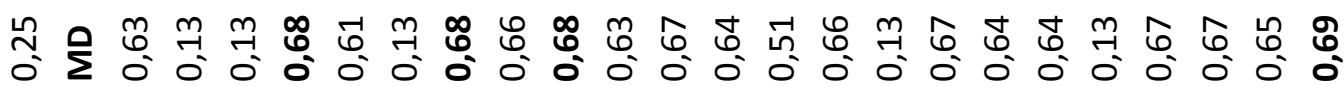

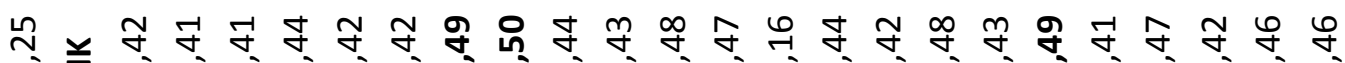

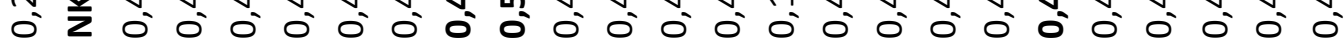

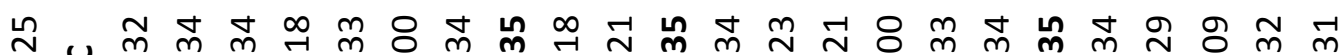

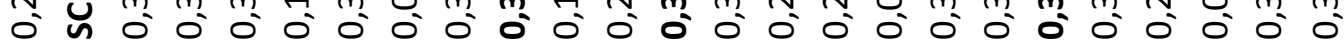
๗

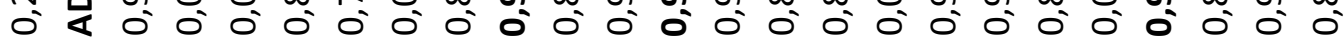

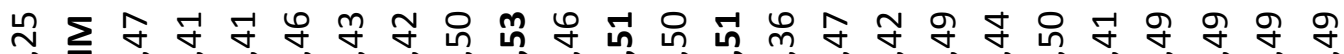

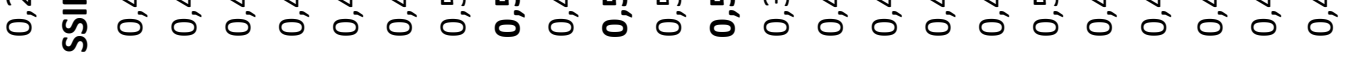

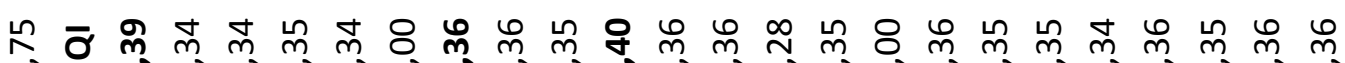

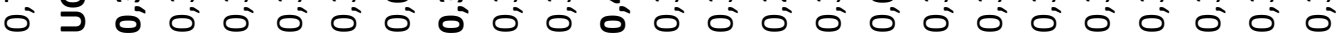

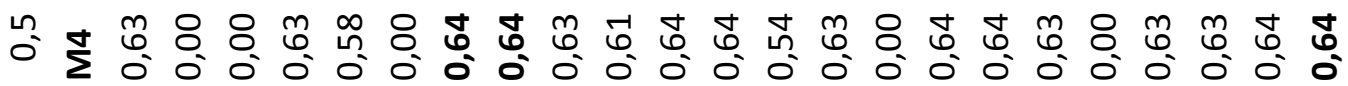

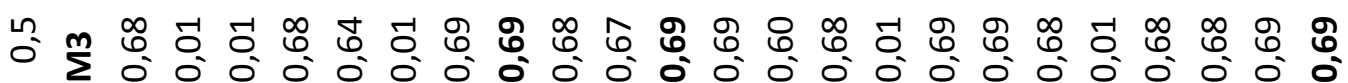
ํ

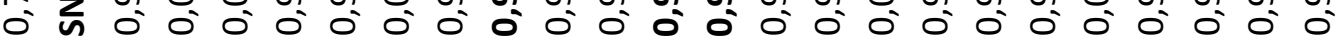
uㅐ

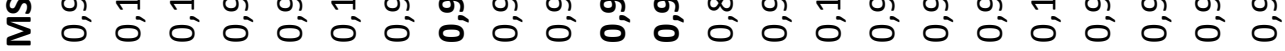

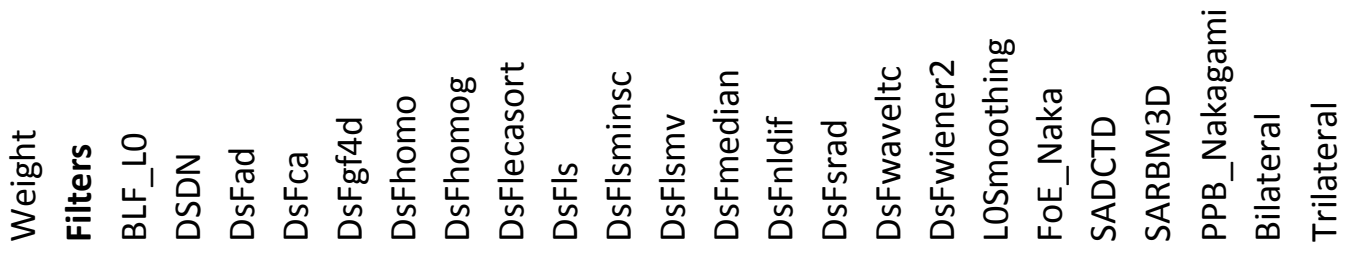




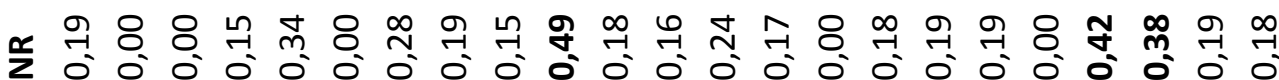

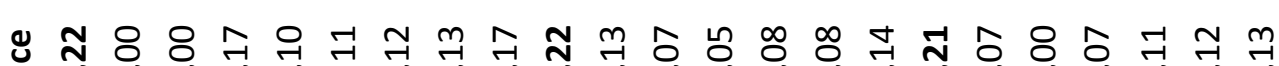

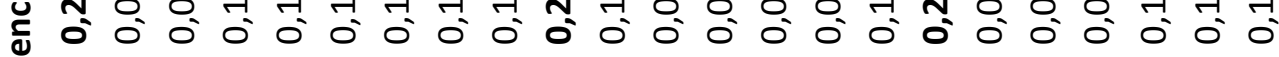
崖

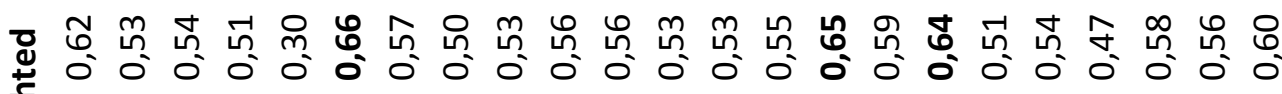
离

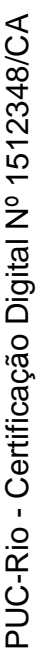

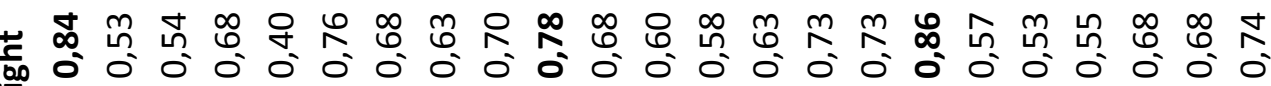

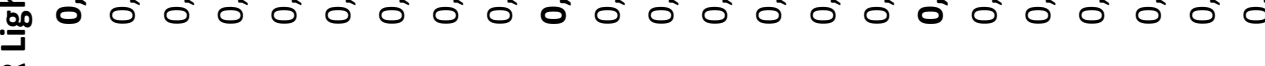
$\stackrel{\propto ⿻}{\alpha}$

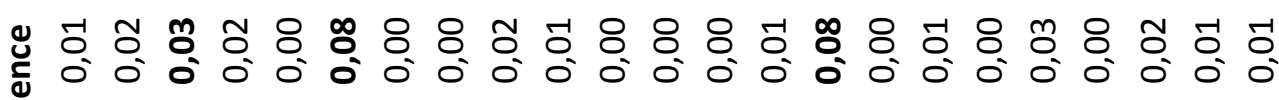
离 芒

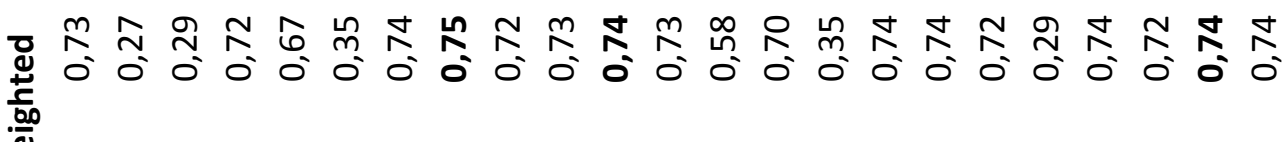
$\stackrel{2}{3}$ 品

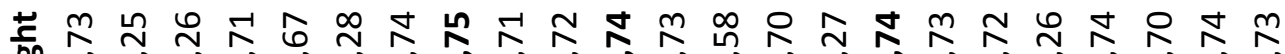

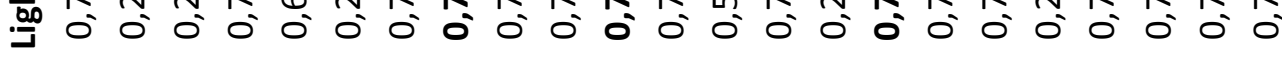
딴

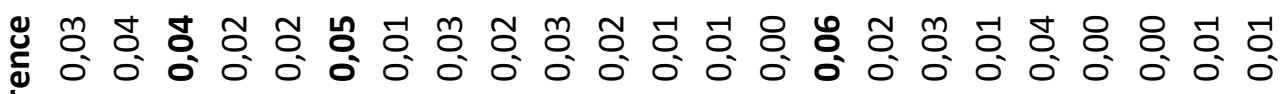
迹

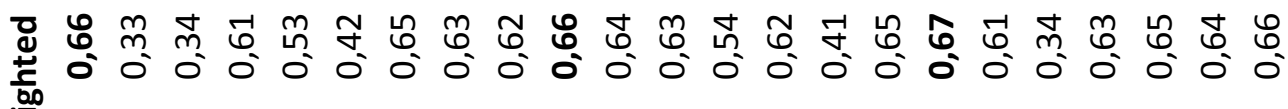

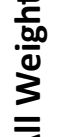

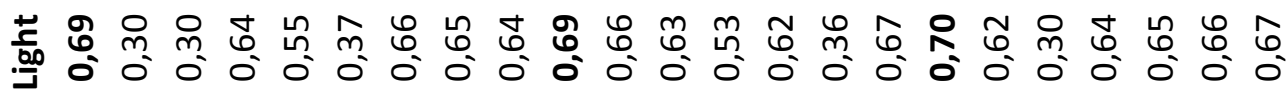
$\overline{\bar{\alpha}}$

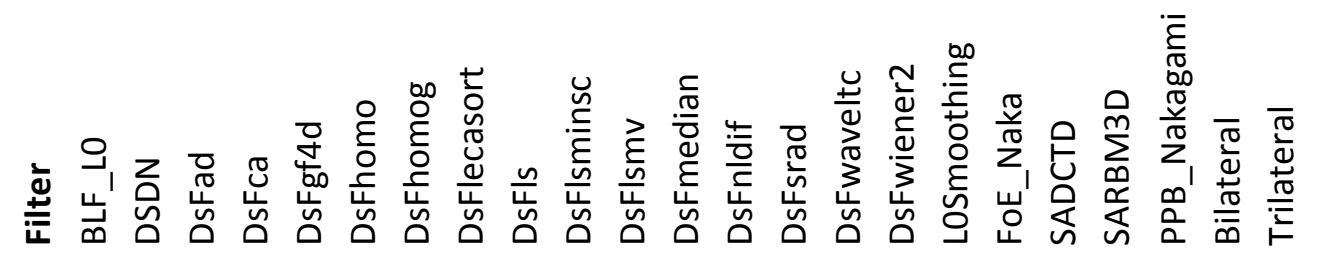




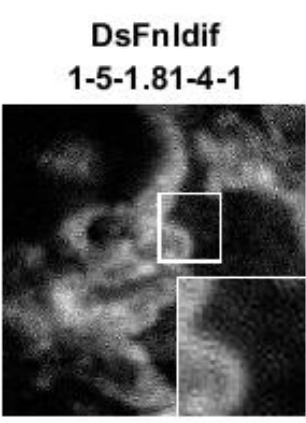

DsFhomo

14-3

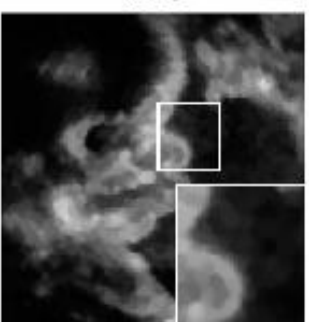

DsFmedian

4-7

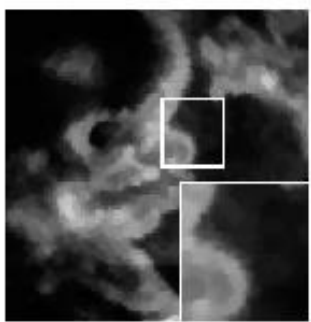

DsFwiener2

3-3

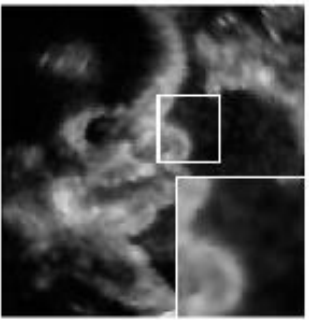

BLF-LO

1-1-5-0.03-9

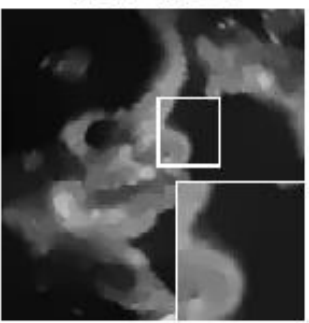

DsFad

1-11-0.05-2

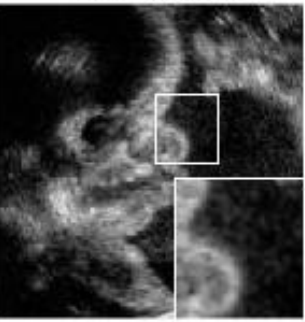

DsFlecasort

2-3

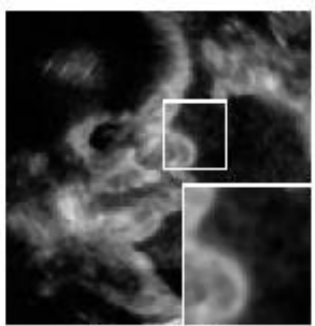

DsFsrad

10-0.71-92.51

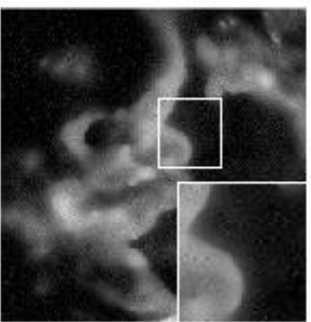

FoE-Naka

8

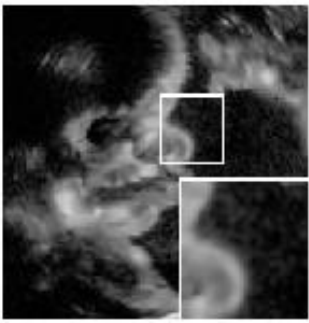

PPB-Nakagami 1-5-12-5-0.901-0.601

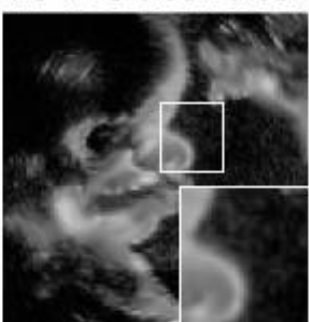

DsFca

3-2

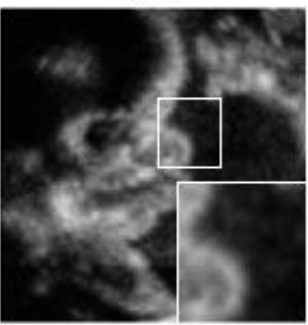

DsFls

2-3

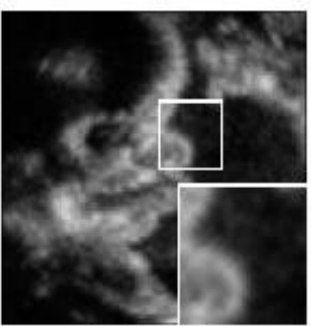

Original

Testcase 02-45

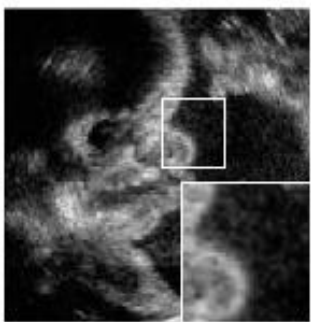

Trilateral

2-1-0.185-0.75

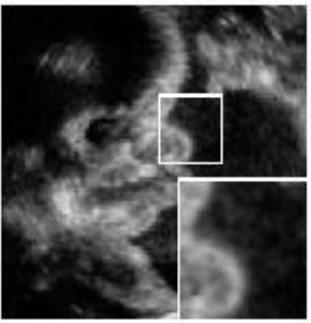

SADCTD

2-0.05

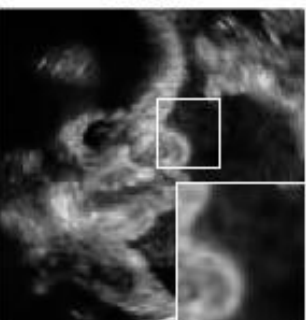

DsFgf4d

11-2

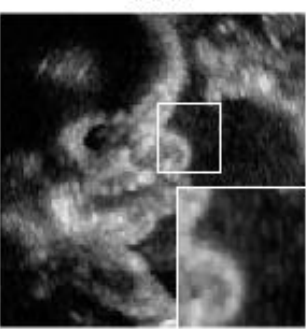

DsFlsminsc

2-5-3

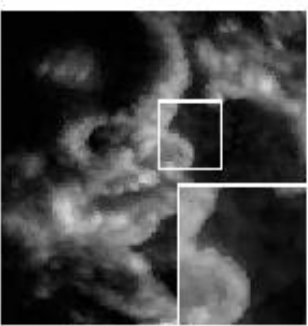

Ground Truth

Testcase 02-45

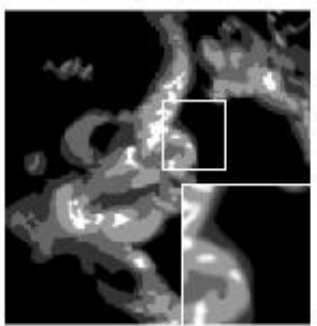

Bilateral

1-3-29

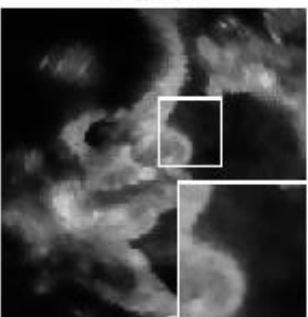

DSDN

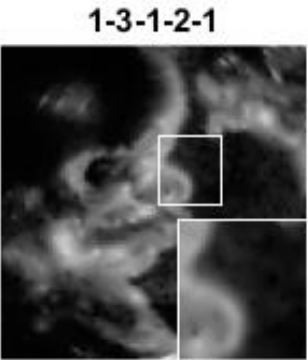

DsFhomog

3-4

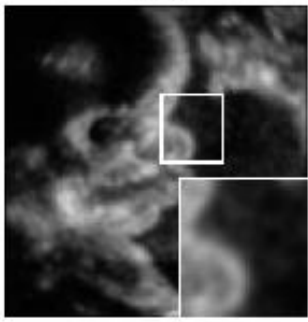

DsFlsmv

3-2

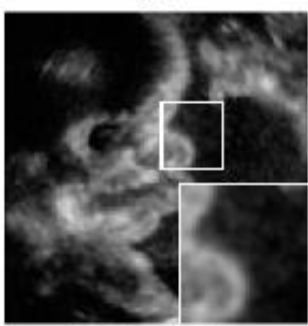

DsFwaveltc

1

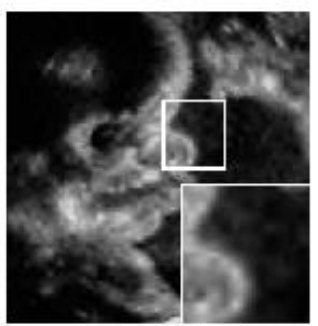

LOSmoothing

1-0.025-8.4

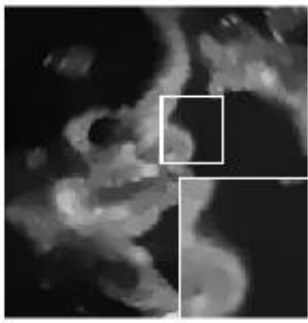

SARBM3D 4-5

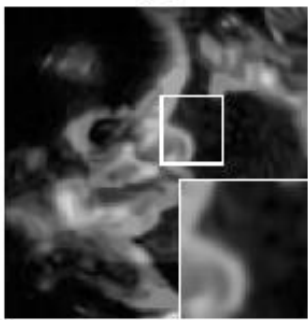

Figura 6.6: Aplicação de todos os filtros apresentados com suas configurações de parâmetros definidas de acordo com as avaliações feitas ao longo do capítulo 6 . No canto inferior direito de cada imagem existe uma área com zoom para que seja possível melhor visualizar o efeito de filtragem obtido. 
ơ ๙ ○ ๙

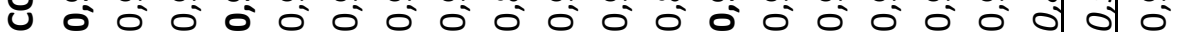

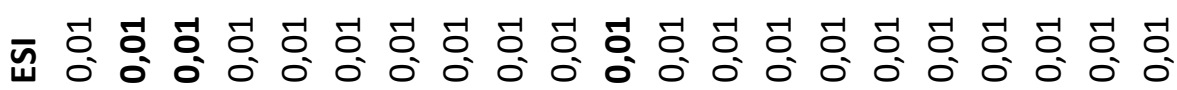

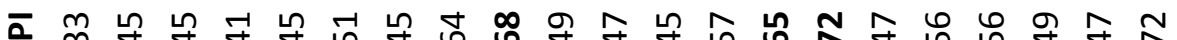

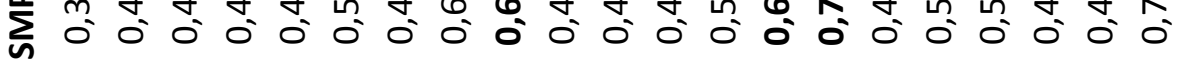

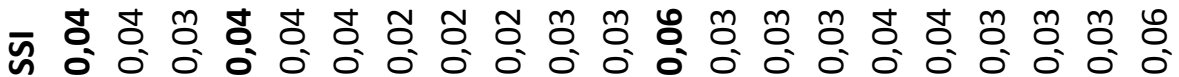
«

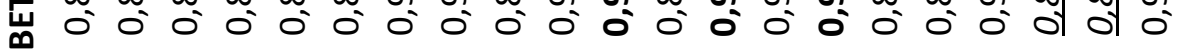
ơ

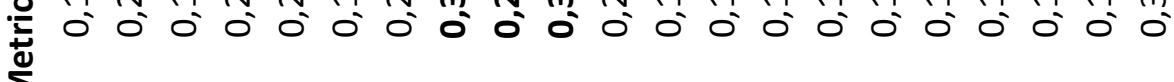

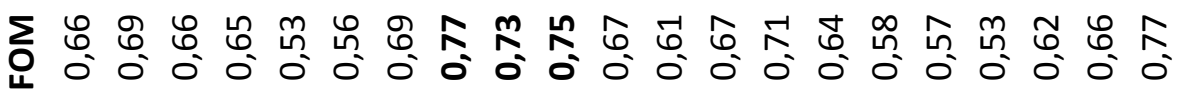
प 山ீ人

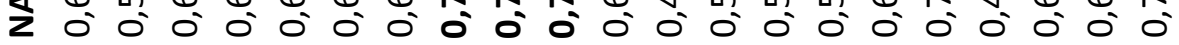

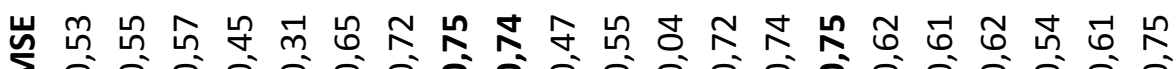

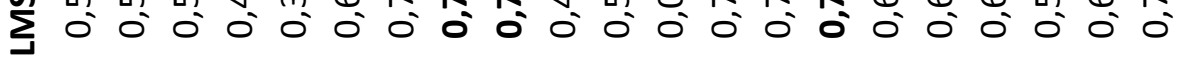

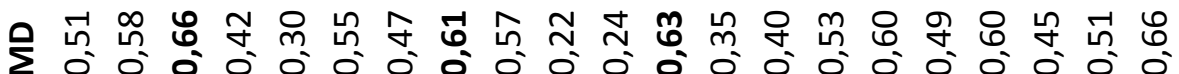

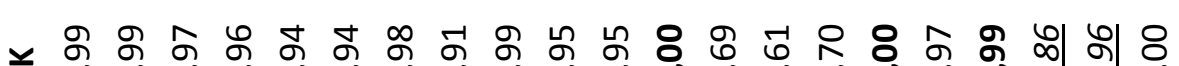
z 누 $\infty$ 人 $⿻ 心 ㇒$

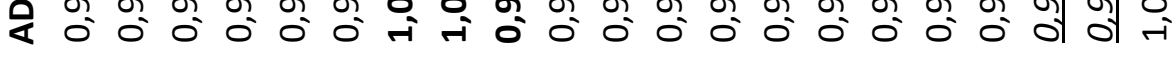
$\sum$ ஸ̊ 의

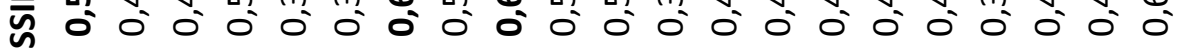

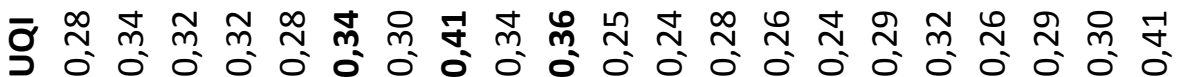

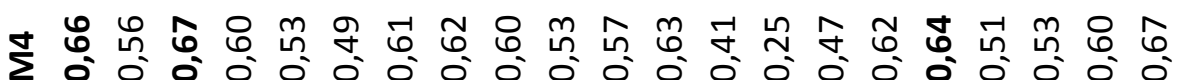

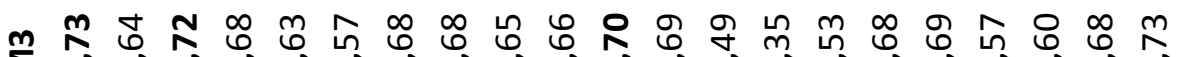

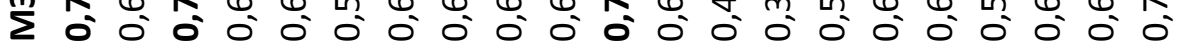

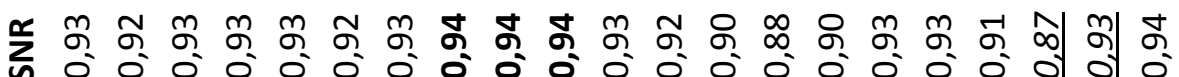

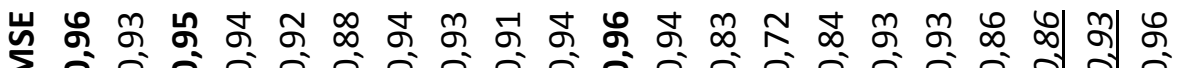

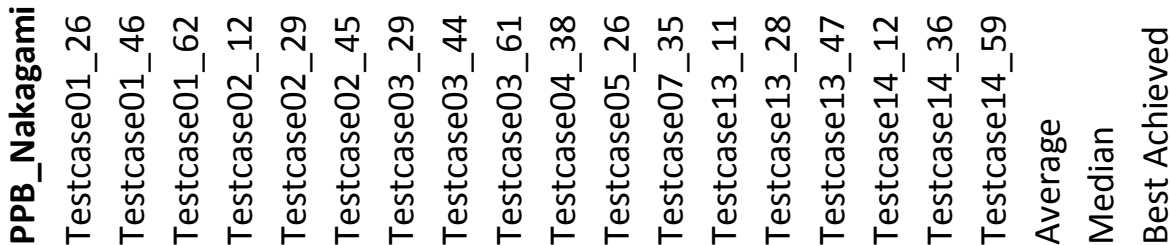




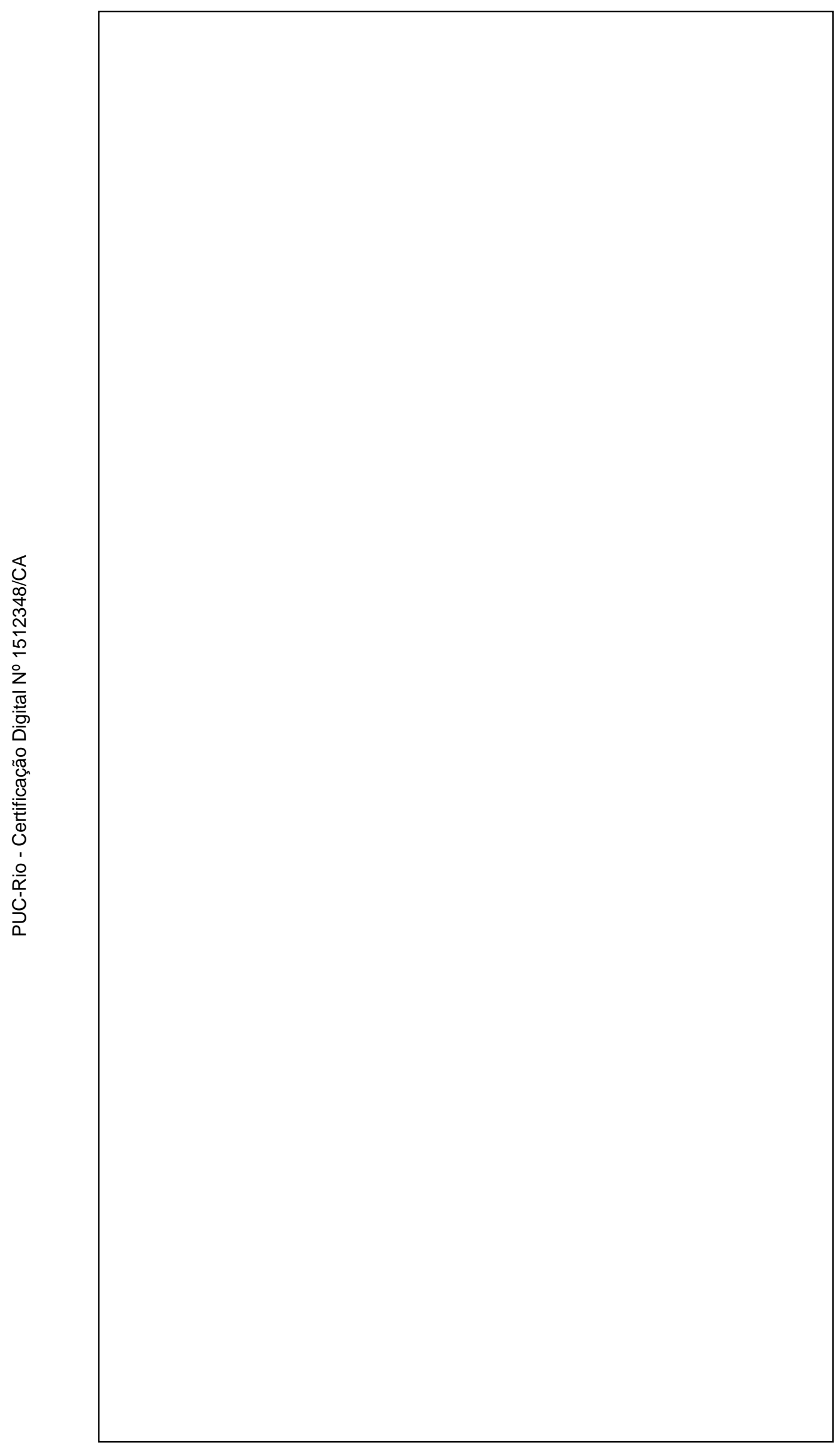




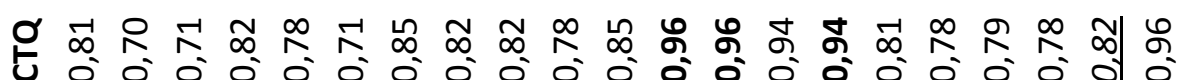
○

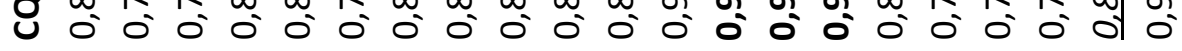

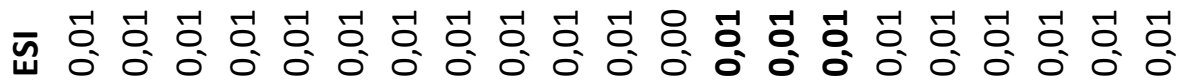
ㄸำ

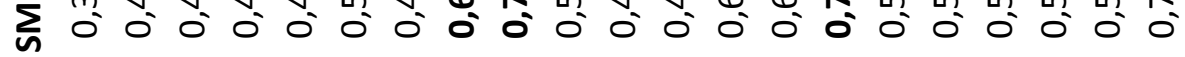
匀 $\varangle \infty$ b

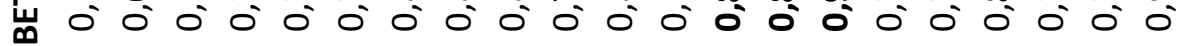

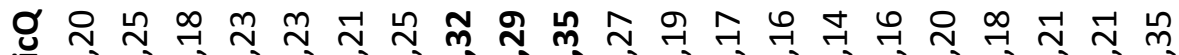

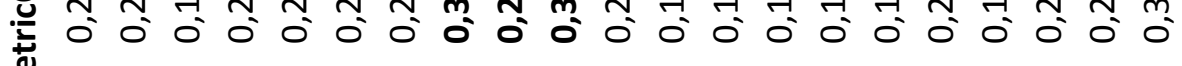
$\sum^{\infty}$

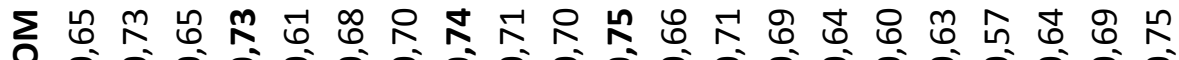

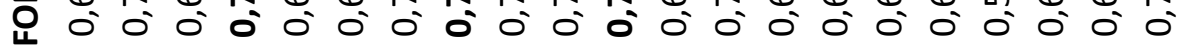
ப 엉

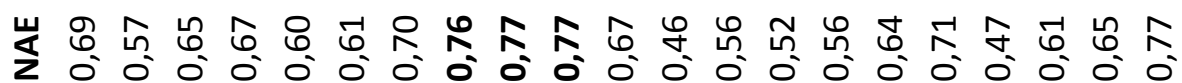
药

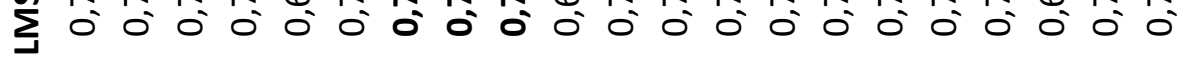

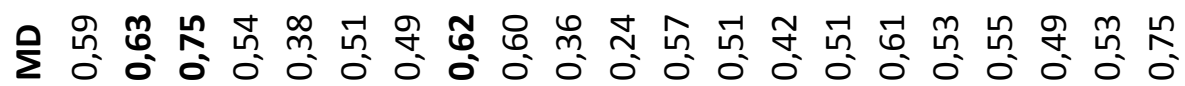

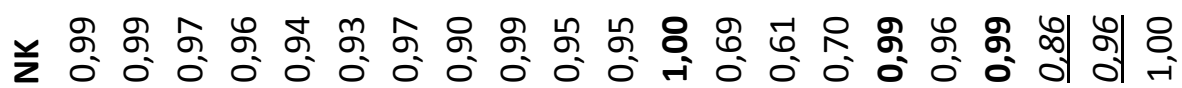
u

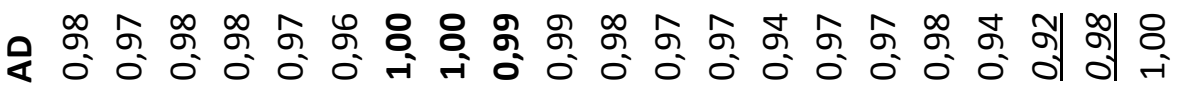

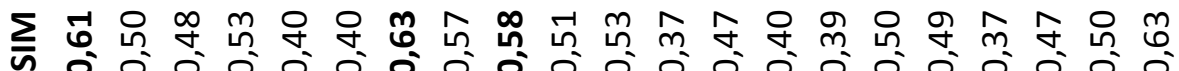

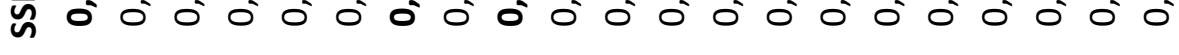

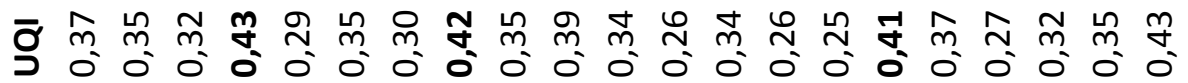

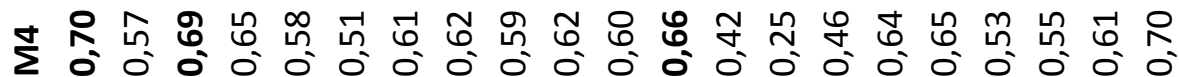

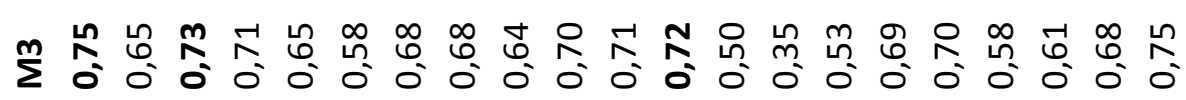

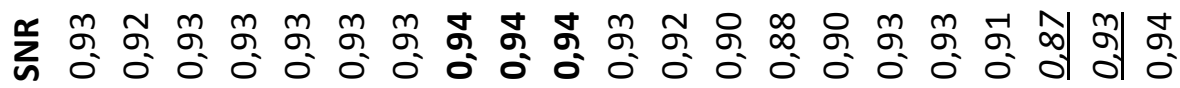

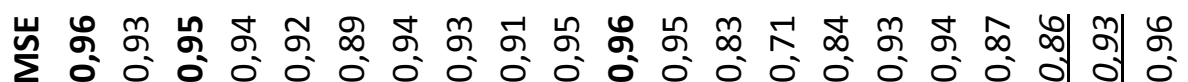

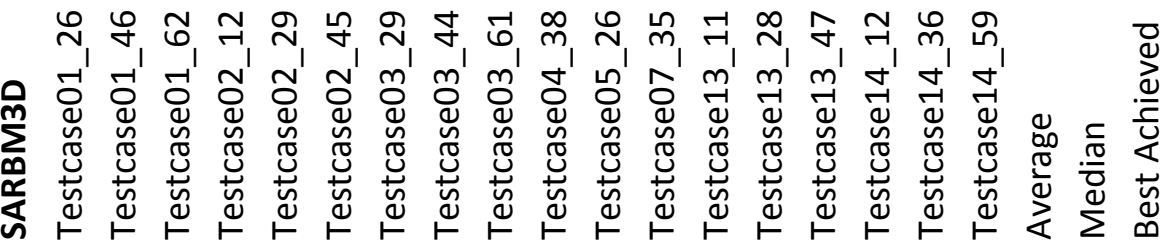




\section{Conclusão e trabalhos futuros}

Neste trabalho, apresentamos diversas comparações entre filtros diferentes a fim de determinar os melhores filtros, e seus parâmetros, que conseguissem atender a uma série de requisitos: preservar/realçar as fronteiras contidas na imagem enquanto ainda removesse o ruído speckle contido em exames de ultrassom e criasse áreas homogêneas onde possível.

Para que a avaliação entre filtros pudesse ser feita, métricas pertencentes a diferentes grupos foram utilizadas, gerando assim uma necessidade de ranquear um resultado em detrimento de outros. Múltiplas tentativas de classificação foram analisadas e testadas, concluindo-se por fim que o método mais robusto de análise foi o de clusterização por $k$-means com o total de clusters $k$ calculado pelo método empírico; seleção de parâmetros resultante da escolha de centroide por Cardinalidade Relativa; com somente as métricas do grupo Full Reference e sem pesos atribuídos às métricas escolhidas.

A avaliação automática dos resultados obtidos, entretanto, não é definitiva. Portanto, uma avaliação visual final foi feita para confirmar que o conjunto de parâmetros escolhido era de fato relevante e para por fim selecionar quais filtros atendem de melhor maneira as características desejadas, dentro do universo de testes realizado.

Os filtros que obtiveram melhor desempenho neste trabalho foram o PPB_Nakagami e o SADCTD, apesar do BLF_L0, DSDN, L0, FoE_Naka e SARBM3D também apresentarem bons resultados. O restante dos filtros falharam em criar resultados visuais tão bons quanto os apresentados pelos filtros escolhidos ou em seguir os Requisitos Fundamentais e Características Desejáveis.

Como os filtros escolhidos são ótimos candidatos em campos diferentes de atuação, um na homogeinização da imagem e o outro em remover o ruído speckle, respectivamente, o uso consecutivo destes poderá acarretar em um resultado ainda melhor. O SADCTD deve ser usado primeiro, impedindo assim que a homogeinização provida pelo PPB_Nakagami aconteça em regiões inadequadas.

Tanto os filtros escolhidos quanto os que apresentaram bons resultados tiveram seus parâmetros apresentados.

Dentre os filtros DsF, apresentados por Loizou[2], o DsFlsmv, DsFhomog e DsFhomo obtiveram os melhores resultados, contrastando com as conclusões obtidas pelo autor, que afirma que os filtros DsFlsmv, DsFlminsc e DsFgf4d teriam obtido o melhor desempenho. Um dos possíveis motivos para esta diferença se dá no fato que as avaliações empregadas foram distintas e que o domínio das imagens de ultrassom testadas é diferente, onde Loizou utilizou imagens corrompidas artificialmente, imagens puramente artificiais e imagens de ultrassom cardíacas ou da artéria carótida.

Vale ressaltar que, apesar do estudo apresentado por Loizou ter sido a base deste trabalho, aqui foram apresentados novos filtros e métricas, além de um estudo completo acerca de como analisar o resultado das métricas, determinar quais delas de fato exprimem as melhorias que foram buscadas neste projeto e como definir, fazendo uso de um framework coerente de testes, os melhores parâmetros para cada filtro.

Apesar do foco deste trabalho não ter sido a avaliação da performance de cada método abordado, diversas otimizações foram realizadas, de forma que são muitas 
as partes em que o código de teste funciona em um ambiente multithread, enquanto o cálculo de alguns filtros e métricas é ainda feito em GPU. Entretanto o ambiente utilizado, o Matlab, apesar de possibilitar a prototipação agilizada dos testes, carece em apresentar performances como as vistas em um ambiente $\mathrm{C}++$, portanto a utilização de módulos $\mathrm{C}++$, com processamento em placas gráficas, diminuiria drasticamente o tempo total de execução.

O método de clusterização adotado também possui espaço para melhorias, visto que o uso do Critério de Gap para a escolha de parâmetros do $k$-means é amplamente mais custoso que o cálculo dos clusters em si e o método empírico apresentado não leva em consideração o comportamento dos dados. Além disso métodos mais recentes foram desenvolvidos, como o DBSCAN[67], capaz de gerar grupos de qualquer formato, ao contrário do $k$-means que assume uma convexidade no agrupamento. Ainda sobre o método de agrupamento, outro teste possível seria o da escolha do melhor cluster pelo centro de massa de seu volume, uma vez que assim regiões de maior densidade seriam um fator mais decisivo na escolha dos centroides, enquanto regiões mais esparsas teriam pouca influência.

Um refinamento do intervalo inicial dos parâmetros usados para cada filtro também pode ser feito ao utilizar os limites dos cluster de maior Cardinalidade Relativa como um novo intervalo, podendo assim melhorar os resultados apresentados enquanto ainda diminuindo o custo computacional sucessivamente a cada refinamento ao restringir gradativamente o intervalo escolhido.

Por fim, as avaliações e resultados apresentados neste trabalho pretenderam criar uma base de comparação sólida para que novos filtros possam ser facilmente comparados com aqueles já existentes na literatura.

Os filtros, métricas e avaliações que obtiveram melhor desempenho podem ainda serem usados num escopo diferente do apresentado neste trabalho, auxiliando métodos de pós-processamento como segmentação, detecção de características, classificação, reconstrução 3D, entre outros, sendo assim uma etapa importante para que estes possam exibir melhores resultados. 


\section{Referências bibliográficas}

[1] Christodoulou, C. I., et al. De-speckle filtering in ultrasound imaging of the carotid artery. Engineering in Medicine and Biology, 2002. 24th Annual Conference and the Annual Fall Meeting of the Biomedical Engineering Society EMBS/BMES Conference, 2002. Proceedings of the Second Joint. Vol. 2. IEEE, 2002.

[2] Loizou, Christos P., and Constantinos S. Pattichis. Despeckle Filtering for Ultrasound Imaging and Video, Volume II: Selected Applications. Synthesis Lectures on Algorithms and Software in Engineering 7.2 (2015): 1-180.

[3] Hiremath, P. S., Prema T. Akkasaligar, and Sharan Badiger. Speckle noise reduction in medical ultrasound images. Advancements and Breakthroughs in Ultrasound Imaging. Gunarathne G.(ed.) InTech (2011): 201-241.

[4] Wilhjelm, Jens E., et al. Visual and quantitative evaluation of selected image combination schemes in ultrasound spatial compound scanning. IEEE transactions on medical imaging 23.2 (2004): 181-190.

[5] Powers, Jeff, and Frederick Kremkau. Medical ultrasound systems. Interface focus 1.4 (2011): 477-489.

[6] Damerjian, V., et al. Speckle characterization methods in ultrasound images-A review. IRBM 35.4 (2014): 202-213.

[7] Goodman JW. Some fundamental properties of speckle. JOSA 1976;66(11):1145-50.

[8] Tuthill TA, Sperry RH, Parker KJ. Deviations from Rayleigh statistics in ultrasonic speckle. Ultrason Imaging 1988;10(2):81-9.

[9] Marti R, Marti J, Freixenet J, Vilanova JC, Barceló J. Robust speckle detection in ultrasound images: evaluation aspects. In: Eigth International Conference on Quality Control by Artificial Vision. 2007.

[10] Bernard O, Touil B, Gelas A, Prost R, Friboulet D. Segmentation of myocardial regions in echocardiography using the statistics of the radiofrequency signal. Funct Imaging Modeling Heart 2007:433-42.

[11] Ghofrani S, Jahed-Motlagh MR, Ayatollahi A. An adaptive speckle suppression filter based on Nakagami distribution. In: EUROCON'2001, Trends in Communications, International Conference. 2001. p. 84-7.

[12] Raju BI, Srinivasan MA. Statistics of envelope of high-frequency ultrasonic backscatter from human skin in vivo. IEEE Trans Ultrason Ferroelectr Freq Control 2002;49(7):871-82.

[13] Wachowiak MP, Smolková R, Zurada JM, Elmaghraby AS. Estimation of $\mathbf{K}$ distribution parameters using neural networks. IEEE Biomed Eng 2002;49(6):617-20.

[14] Wagner RF, Smith SW, Sandrik JM, Lopez H. Statistics of speckle in ultrasound B-scans. IEEE Trans Sonics Ultrason 1983;30(3):156-63.

[15] Hao X, Bruce CJ, Pislaru C, Greenleaf JF. Characterization of reperfused infarcted myocardium from high-frequency intracardiac ultrasound imaging using homodyned $\mathbf{K}$ distribution. IEEE Trans Ultrason Ferroelectr Freq Control 2002;49(11):1530-42. 
[16] Lee, Jong-Sen. Digital image enhancement and noise filtering by use of local statistics. IEEE transactions on pattern analysis and machine intelligence 2 (1980): 165-168.

[17] Frost, Victor S., et al. A model for radar images and its application to adaptive digital filtering of multiplicative noise. IEEE Transactions on pattern analysis and machine intelligence 2 (1982): 157-166.

[18] Kuan, Darwin T., et al. Adaptive noise smoothing filter for images with signal-dependent noise. IEEE transactions on pattern analysis and machine intelligence 2 (1985): 165-177.

[19] P. Rerona and J. Malik, Scale-space and edge detection using anisotropic diffusion. IEEE Trans. Pattern Anal. Mach. Intell., vol. 12, no. 7, pp. 629-639, July 1990.

[20] Di Martino, Gerardo, et al. Benchmarking framework for SAR despeckling. IEEE Transactions on Geoscience and Remote Sensing 52.3 (2014): 1596-1615.

[21] Coupé, Pierrick, et al. Nonlocal means-based speckle filtering for ultrasound images. IEEE transactions on image processing 18.10 (2009): 2221-2229.

[22] K. Dabov, A. Foi, V. Katkovnik, and K. Egiazarian, Image denoising by sparse 3D transform-domain collaborative filtering. IEEE Trans. Image Process., vol. 16, no. 8, pp. 2080-2095, Aug. 2007.

[23] Feng, Hongxiao, Biao Hou, and Maoguo Gong. SAR image despeckling based on local homogeneous-region segmentation by using pixelrelativity measurement. IEEE Transactions on Geoscience and Remote Sensing 49.7 (2011): 2724-2737.

[24] Peterson, Jason D. Clustering overview. 2008]. http://www. cs. ndsu. nodak. edu/ jasonpet/CSCI779/Clustering. pdf (2002).

[25] Nagao, Makoto, and Takashi Matsuyama. Edge preserving smoothing. Computer graphics and image processing 9.4 (1979): 394-407.

[26] Nieminen, Ari, Pekka Heinonen, and Yrjo Neuvo. A new class of detailpreserving filters for image processing. IEEE Transactions on Pattern Analysis and Machine Intelligence 1 (1987): 74-90.

[27] Frost, Victor S., et al. A model for radar images and its application to adaptive digital filtering of multiplicative noise. IEEE Transactions on pattern analysis and machine intelligence 2 (1982): 157-166.

[28] R. Gonzalez and R. Woods, Digital image processing, 2nd ed. AddisonWesley Longman Publishing Co., Inc. Boston, MA, USA, 2002.

[29] S.M. Ali and R.E. Burge, New automatic techniques for smoothing and segmenting SAR images. Signal Processing, North-Holland, vol. 14, pp. 335-346, 1988, doi:10.1016/0165-1684(88)90092-8.

[30] L. Busse, T. R. Crimmins, and J. R. Fienup, A model based approach to improve the performance of the geometric filtering speckle reduction algorithm. in Proc. IEEE Ultrason. Symp., 1995, pp. 1353-1356.

[31] Foi, A., R. Bilcu, V. Katkovnik, and K. Egiazarian, Anisotropic local approximations for pointwise adaptive signal-dependent noise removal. Proc. XIII European Signal Process. Conf., EUSIPCO 2005, September 2005.

[32] Foi, A., V. Katkovnik, and K. Egiazarian, Pointwise Shape-Adaptive DCT for High-Quality Denoising and Deblocking of Grayscale and 
Color Images. IEEE Trans. Image Process., vol. 16, no. 5, pp. 13951411, May 2007. DOI: http://doi.org/10.1109/TIP.2007.891788

[33] Foi, A., K. Dabov, V. Katkovnik, and K. Egiazarian, Shape-Adaptive DCT for Denoising and Image Reconstruction. Proc. SPIE Electronic Imaging 2006, Image Processing: Algorithms and Systems V, 6064A-18, San Jose, January 2006.

[34] Y. Yongjian and S.T. Acton, Speckle reducing anisotropic diffusion. IEEE Trans. Image Process., vol. 11, no. 11, pp. 1260-1270, November 2002, doi:10.1109/TIP.2002.804276.

[35] K. Abd-Elmoniem, A.-B. Youssef, and Y. Kadah, Real-time speckle reduction and coherence enhancement in ultrasound imaging via nonlinear anisotropic diffusion. IEEE Trans. Biomed. Eng., vol. 49, no. 9, pp. 997-1014, Sept. 2002, doi:10.1109/TBME.2002.1028423.

[36] K.N. Chaudhury, D. Sage, and M. Unser, Fast O(1) bilateral filtering using trigonometric range kernels. IEEE Trans. Image Processing, vol. 20, no. 11, 2011.

[37] K.N. Chaudhury, Acceleration of the shiftable O(1) algorithm for bilateral filtering and non-local means. IEEE Transactions on Image Proc., vol. 22, no. 4, 2013.

[38] S. Ghosh and K.N. Chaudhury, On fast bilateral filtering using Fourier kernels. IEEE Signal Processing Letters, vol. 23, no. 5, pp. 570-573, 2016.

[39] Choudhury, Prasun, and Jack Tumblin. The trilateral filter for high contrast images and meshes. ACM SIGGRAPH 2005 Courses. ACM, 2005.

[40] Chen, Yunjin, et al. A higher-order MRF based variational model for multiplicative noise reduction. Signal Processing Letters, IEEE 21.11 (2014): 1370-1374.

[41] P. Ochs, Y. Chen, T. Brox, and T. Pock. iPiano: Inertial Proximal Algorithm for Nonconvex Optimization. SIAM Journal on Imaging Sciences, 7(2):1388-1419, 2014.

[42] $\mathrm{Xu}, \mathrm{Li}$, et al. Image smoothing via $\mathbf{L ~} \mathbf{0}$ gradient minimization. ACM Transactions on Graphics (TOG). Vol. 30. No. 6. ACM, 2011.

[43] Deledalle, Charles-Alban, Loïc Denis, and Florence Tupin. Iterative weighted maximum likelihood denoising with probabilistic patchbased weights. IEEE Transactions on Image Processing 18.12 (2009): 2661-2672.

[44] Parrilli, Sara, et al. A nonlocal SAR image denoising algorithm based on LLMMSE wavelet shrinkage. IEEE Transactions on Geoscience and Remote Sensing 50.2 (2012): 606-616.

[45] Liang, Haoyi, and Daniel S. Weller. Comparison-based Image Quality Assessment for Parameter Selection. arXiv preprint arXiv:1601.04619(2016).

[46] Jensen, Jørgen Arendt. Simulation of advanced ultrasound systems using Field II. Biomedical Imaging: Nano to Macro, 2004. IEEE International Symposium on. IEEE, 2004.

[47] Vora, V. S., et al. Analysis of Compressed Image Quality Assessments. M. Tech Student in E \&C Dept, CCET, WadhwanGujarat (2010). 
[48] Chen, Tzong-Jer, et al. A novel image quality index using Moran I statistics. Physics in Medicine and Biology 48.8 (2003): N131.

[49] Winkler, Stefan. Vision models and quality metrics for image processing applications. These de doctorat, École Polytechnique Fédérale de Lausanne (2000).

[50] Wang, Zhou, et al. Image quality assessment: from error visibility to structural similarity. IEEE transactions on image processing 13.4 (2004): 600-612.3

[51] Sakrison, D. On the role of the observer and a distortion measure in image transmission. IEEE Transactions on Communications 25.11 (1977): 1251-1267.

[52] Z. Wang, A. Bovik, A universal image quality index. IEEE Signal Processing Letters, vol. 9, pp. 81-84, March 2002

[53] Eskicioglu, Ahmet M., and Paul S. Fisher. Image quality measures and their performance. IEEE Transactions on communications 43.12 (1995): 2959-2965.

[54] Swinscow TDV. In: Statistics at square one. Nineth Edition. Campbell M J, editor. University of Southampton; Copyright BMJ Publishing Group 1997.

[55] Abdou, Ikram E., and William K. Pratt. Quantitative design and evaluation of enhancement/thresholding edge detectors. Proceedings of the IEEE 67.5 (1979): 753-763.

[56] Canny, John. A computational approach to edge detection. IEEE Transactions on pattern analysis and machine intelligence 6 (1986): 679698.

[57] Sattar, Farook, et al. Image enhancement based on a nonlinear multiscale method. IEEE transactions on image processing 6.6 (1997): 888-895.

[58] Sheng, Yongwei, and Zong-Guo Xia. A comprehensive evaluation of filters for radar speckle suppression. Geoscience and Remote Sensing Symposium, 1996. IGARSS'96. 'Remote Sensing for a Sustainable Future.', International. Vol. 3. IEEE, 1996.

[59] Shamsoddini, A. and J. C. Trinder, Image texture preservation in speckle noise suppression. ISPRS TC VII Symposium | 100 Years ISPR, W. Wagner and B. Szkely (eds.), Vol. XXXVIII, Part 7A, 239244, Vienna, Austria, Jul. 5-7, 2010.

[60] Zhang, W., F. Liu, L. Jiao, B. Hou, S.Wang, and R. Shang, SAR image despeckling using edge detection and feature clustering in bandelet domain. IEEE Geoscience and Remote Sensing Letters, Vol. 7, No. 1, 131,135, Jan. 2010.

[61] Zhu, Xiang, and Peyman Milanfar. Automatic parameter selection for denoising algorithms using a no-reference measure of image content. IEEE Transactions on Image Processing 19.12 (2010): 31163132.

[62] Lloyd, Stuart P. Least Squares Quantization in PCM. IEEE Transactions on Information Theory. Vol. 28, 1982, pp. 129-137.

[63] Arthur, David, and Sergi Vassilvitskii. K-means++: The Advantages of Careful Seeding. SODA '07: Proceedings of the Eighteenth Annual ACM-SIAM Symposium on Discrete Algorithms. 2007, pp. 1027-1035. 
[64] Tibshirani, R., G. Walther, and T. Hastie. Estimating the number of clusters in a data set via the gap statistic. Journal of the Royal Statistical Society: Series B. Vol. 63, Part 2, 2001, pp. 411-423.

[65] Han, Jiawei, Jian Pei, and Micheline Kamber. Data mining: concepts and techniques. Elsevier, 2011.

[66] http://datascienceandme.com/topics/octavemyclusters.html.

[67] Ester, Martin, et al. A density-based algorithm for discovering clusters in large spatial databases with noise. Kdd. Vol. 96. No. 34. 1996. 
8. Anexo A

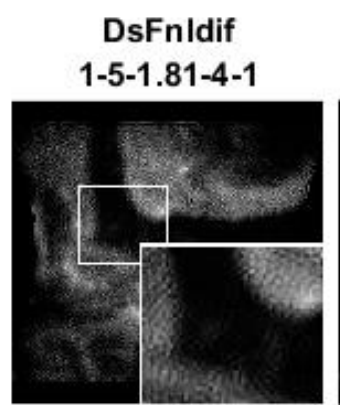

DsFad

1-11-0.05-2

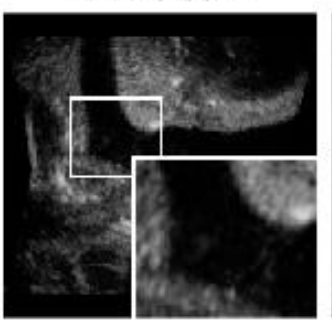

DsFhomo

14-3
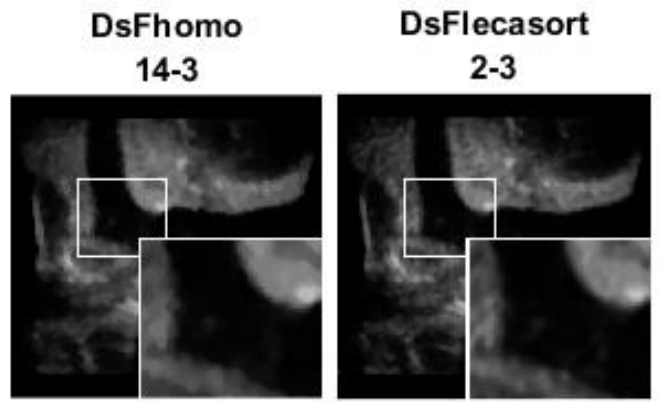

DsFmedian

4-7

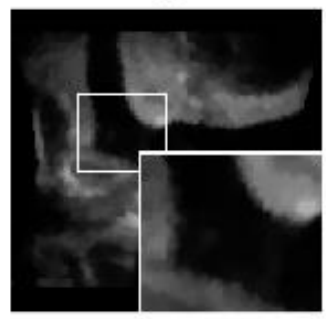

DsFwiener2

3-3

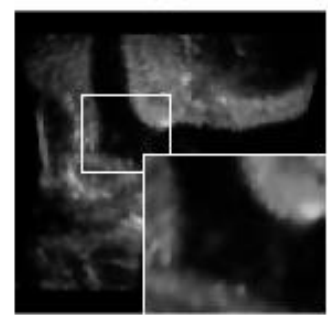

DsFsrad

10-0.71-92.51

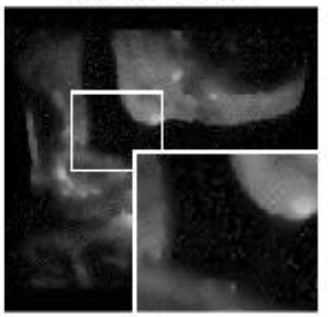

FoE-Naka

8

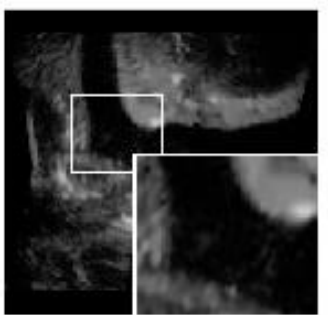

PPB-Nakagami

BLF-LO 1-5-12-5-0.901-0.601
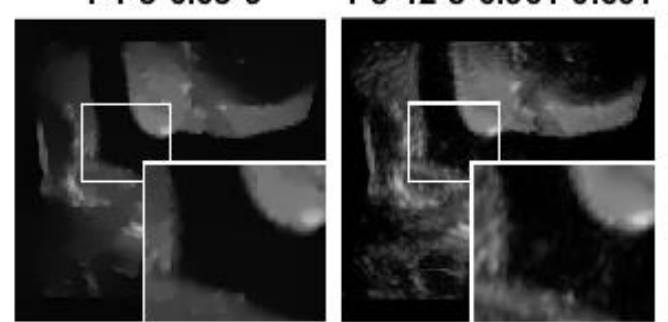

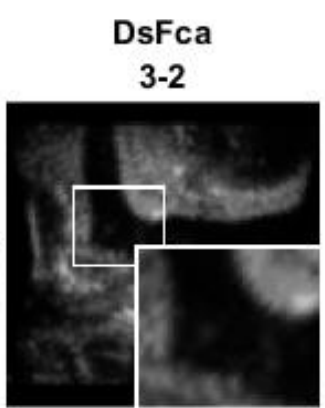

DsFls

2-3

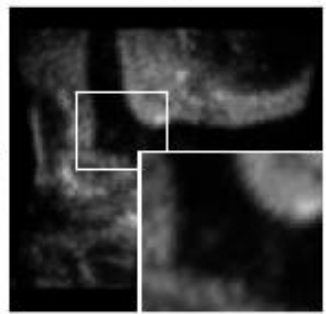

Original

Testcase 01-26

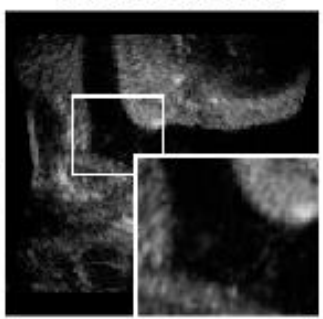

Trilateral

2-1-0.185-0.75

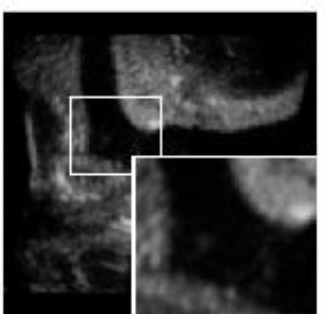

SADCTD

2-0.05

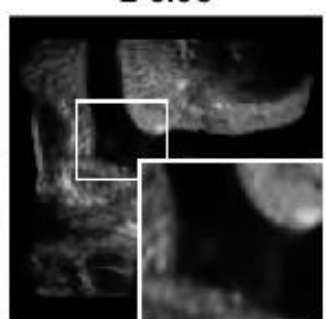

DsFgf4d

11-2

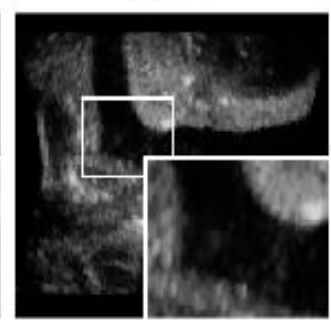

DsFlsminsc

2-5-3

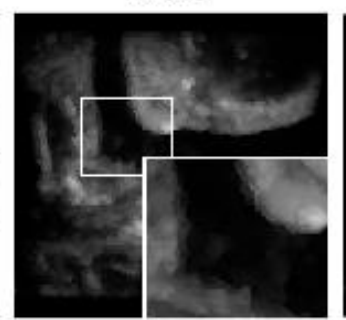

Ground Truth

Testcase 01-26

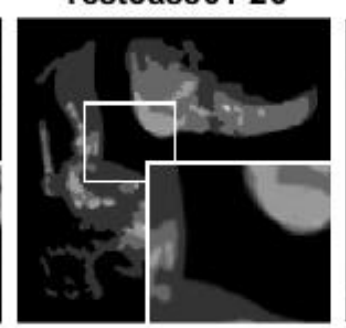

Bilateral

1-3-29

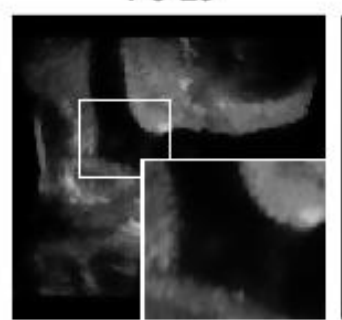

DSDN

1-3-1-2-1

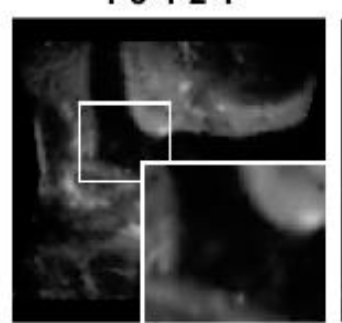

DsFhomog

3-4

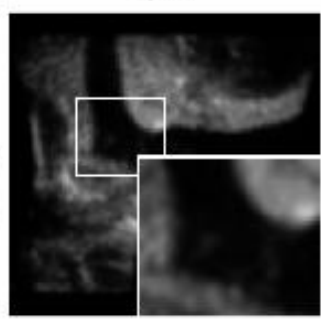

DsFIsmv

3-2

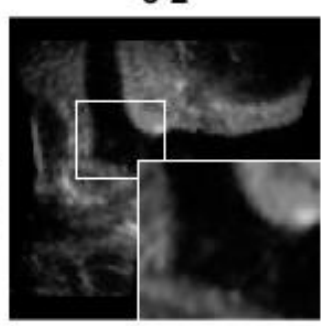

DsFwaveltc

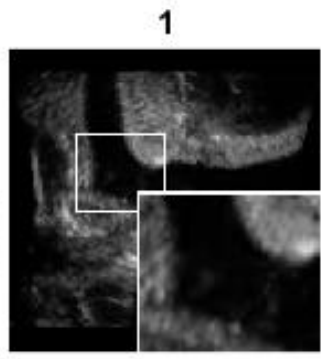

LOSmoothing

1-0.025-8.4

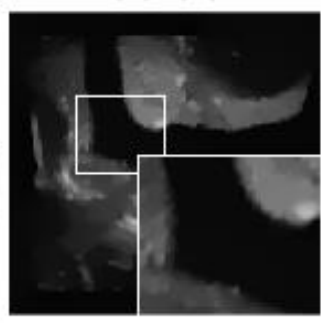

SARBM3D

4-5

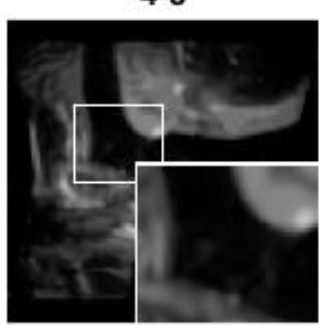

Figura 8.1: Aplicações de todos os filtros discutidos no capítulo 3, com as configurações de parâmetros determinadas na seção 6.9 na imagem de teste Testcase 01-26. No canto inferior direito de cada imagem encontra-se uma região ampliada de uma área de interesse do exame que está sendo analisado. 


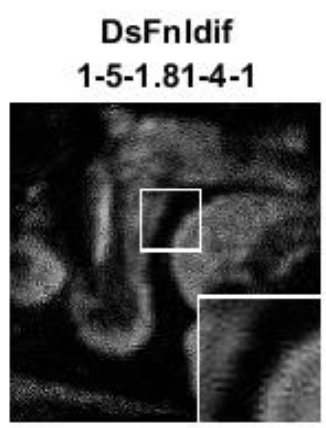

DsFhomo

14-3

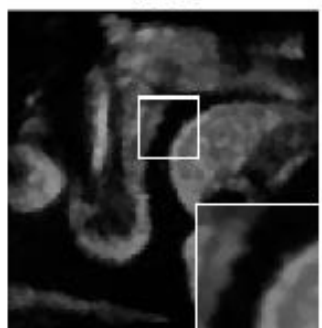

DsFmedian

4-7

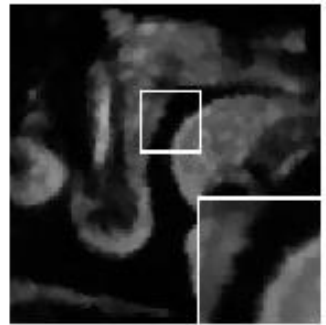

DsFwiener2

3-3

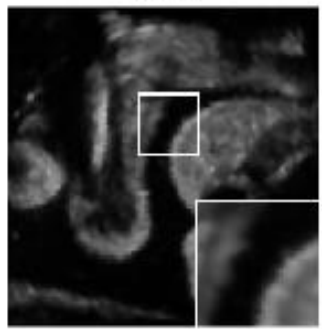

BLF-LO

1-1-5-0.03-9

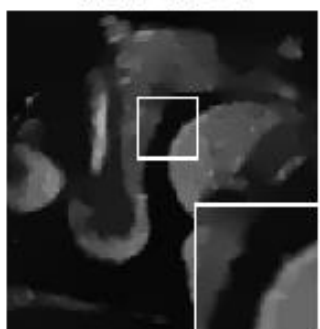

DsFad

1-11-0.05-2

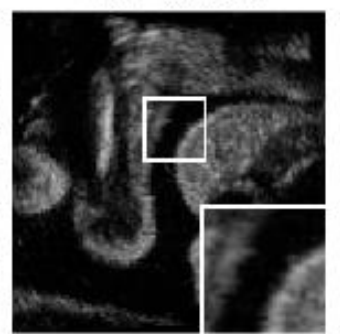

DsFlecasort

2-3

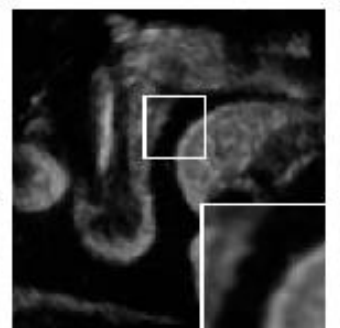

DsFsrad

10-0.71-92.51

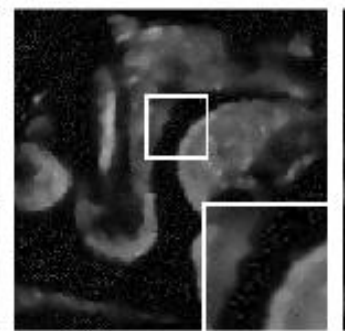

FoE-Naka

8

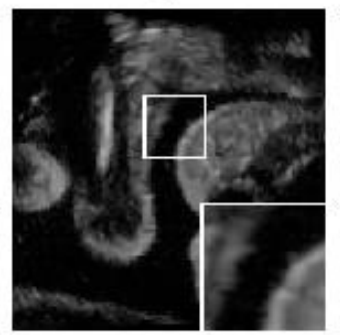

PPB-Nakagami 1-5-12-5-0.901-0.601

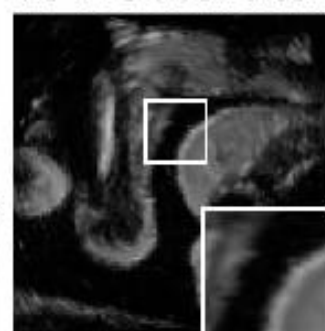

DsFca

3-2

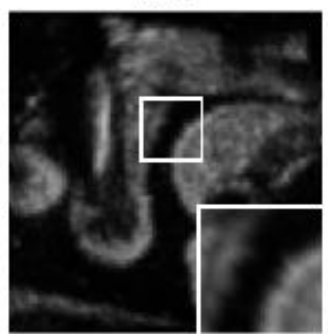

DsFls

2-3

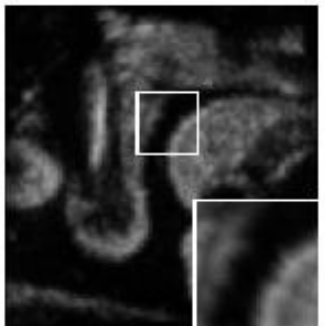

Original

Testcase 01-46

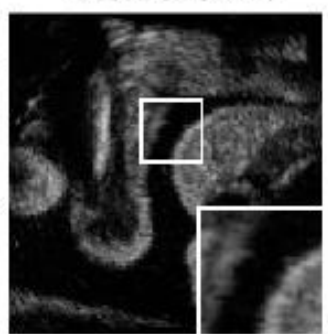

Trilateral

2-1-0.185-0.75

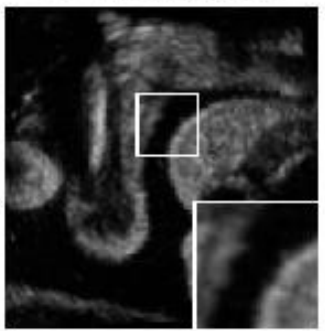

SADCTD

2-0.05

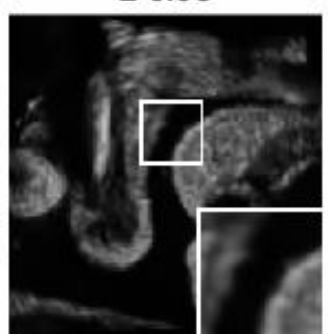

DsFgf4d

11-2

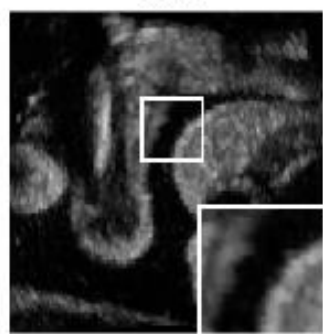

DsFlsminsc

2-5-3

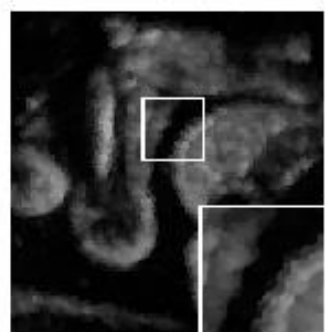

Ground Truth

Testcase 01-46

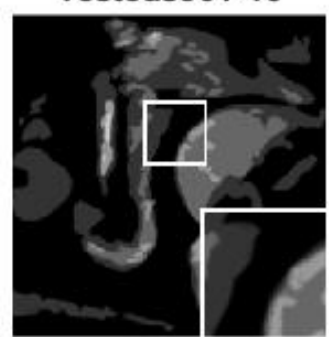

Bilateral

1-3-29

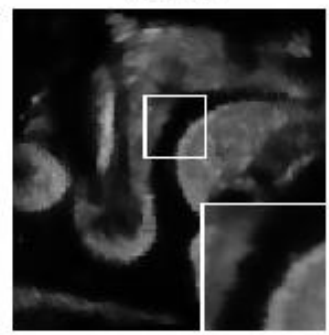

DSDN

1-3-1-2-1

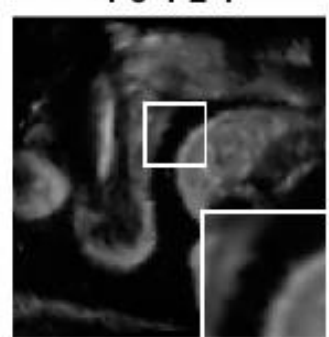

DsFhomog

3-4

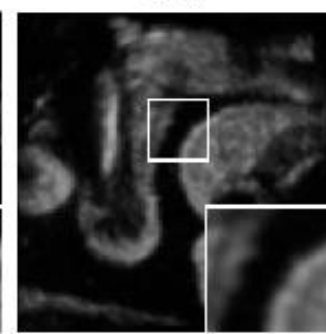

DsFlsmv

3-2

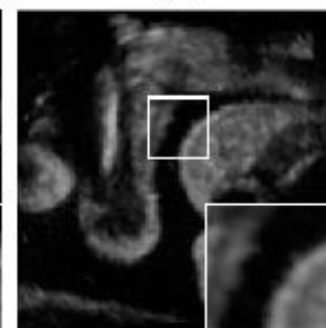

DsFwaveltc

1

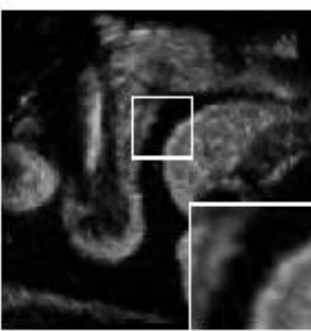

LOSmoothing

$1-0.025-8.4$

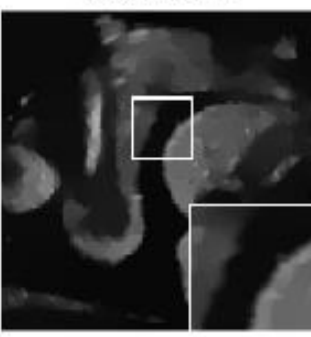

SARBM3D

4-5

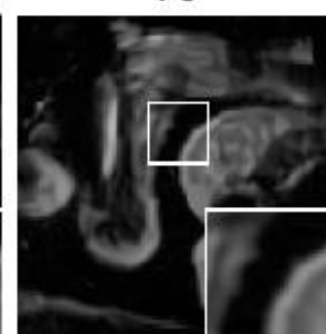

Figura 8.2: Aplicações de todos os filtros discutidos no capítulo 3, com as configurações de parâmetros determinadas na seção 6.9 na imagem de teste Testcase 01-46. No canto inferior direito de cada imagem encontra-se uma região ampliada de uma área de interesse do exame que está sendo analisado. 


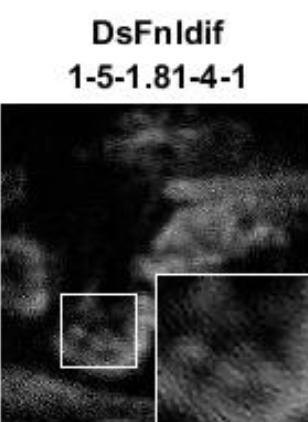

DsFhomo

14-3

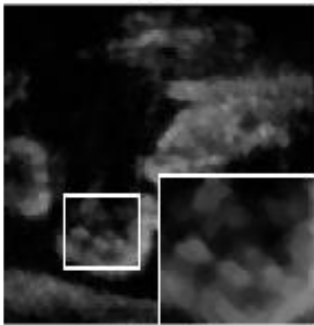

DsFmedian

4-7

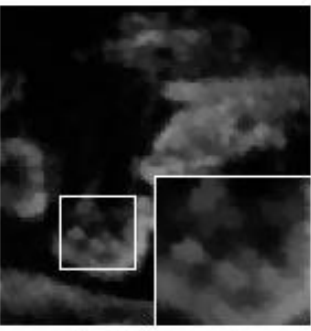

DsFwiener2

3-3

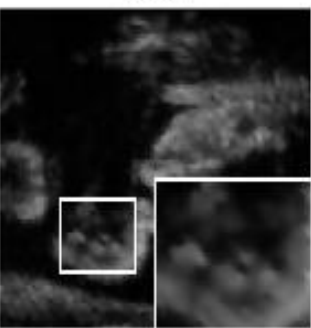

BLF-LO

1-1-5-0.03-9

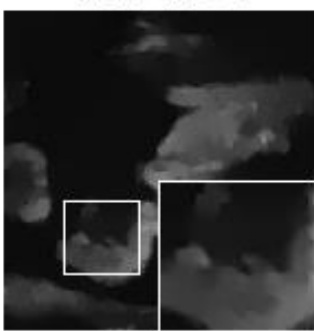

DsFad

1-11-0.05-2

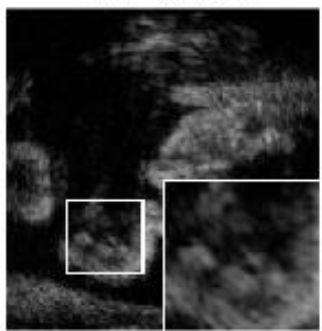

DsFlecasort

2-3

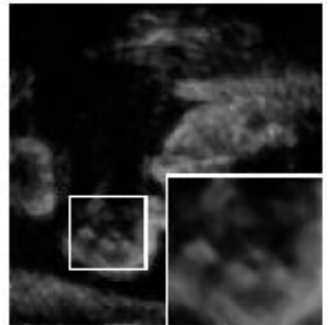

DsFsrad

10-0.71-92.51

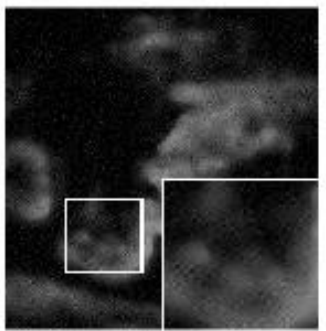

FoE-Naka

8

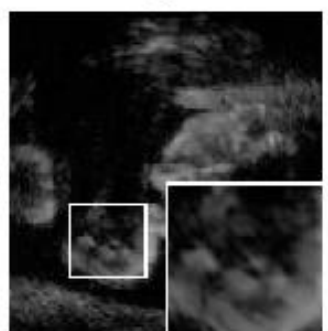

PPB-Nakagami 1-5-12-5-0.901-0.601

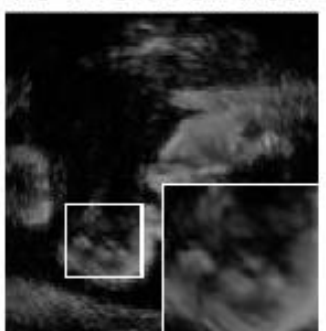

3-2

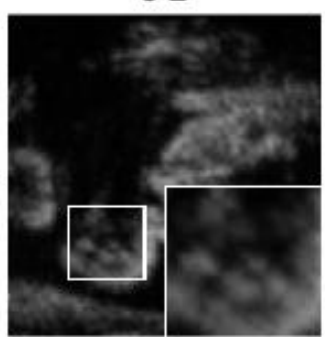

DsFls

2-3

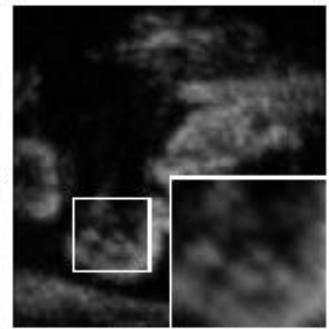

Original

Testcase 01-62

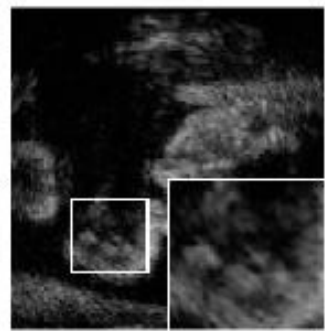

Trilateral

2-1-0.185-0.75

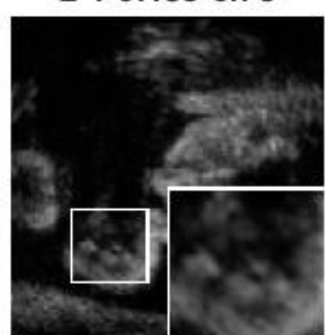

SADCTD

2-0.05

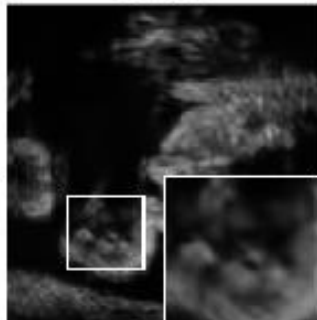

DsFgf4d

11-2

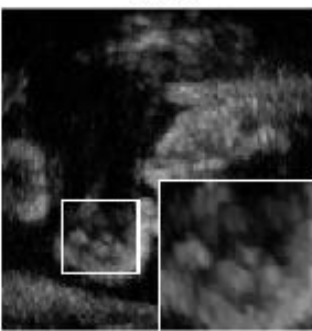

DsFlsminsc

2-5-3

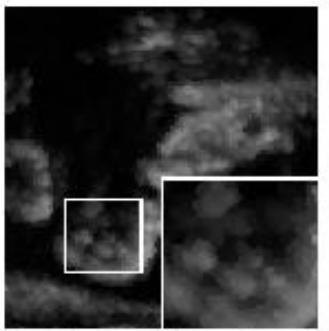

Ground Truth

Testcase 01-62

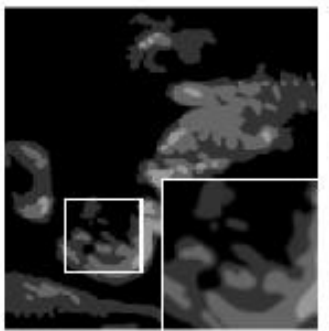

Bilateral

1-3-29

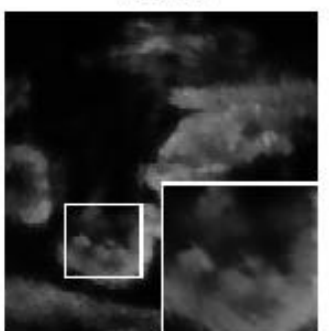

DSDN

1-3-1-2-1

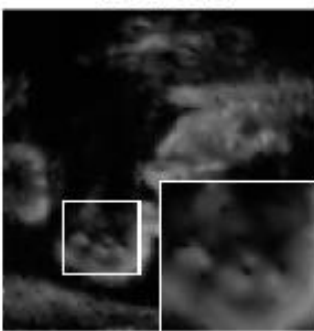

DsFhomog

3-4

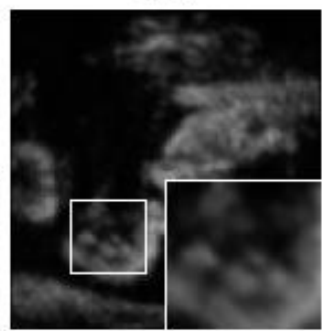

DsFlsmv

3-2

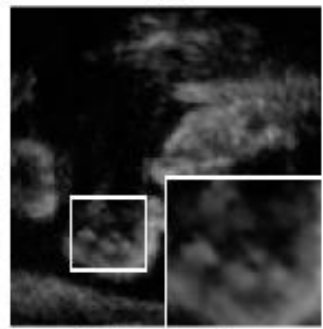

DsFwaveltc

1

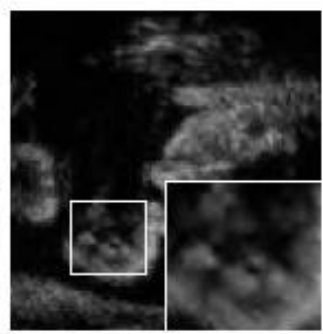

L0Smoothing

1-0.025-8.4

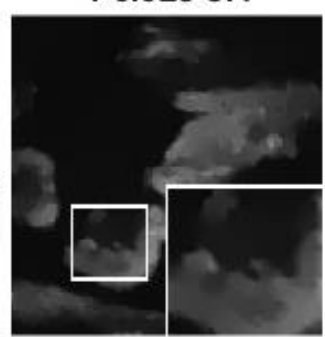

SARBM3D

4-5

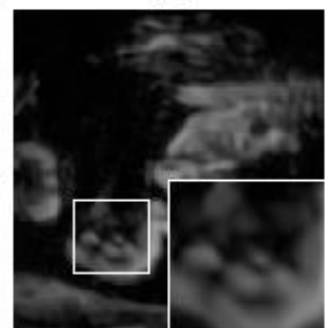

Figura 8.3: Aplicações de todos os filtros discutidos no capítulo 3, com as configurações de parâmetros determinadas na seção 6.9 na imagem de teste Testcase 01-62. No canto inferior direito de cada imagem encontra-se uma região ampliada de uma área de interesse do exame que está sendo analisado. 


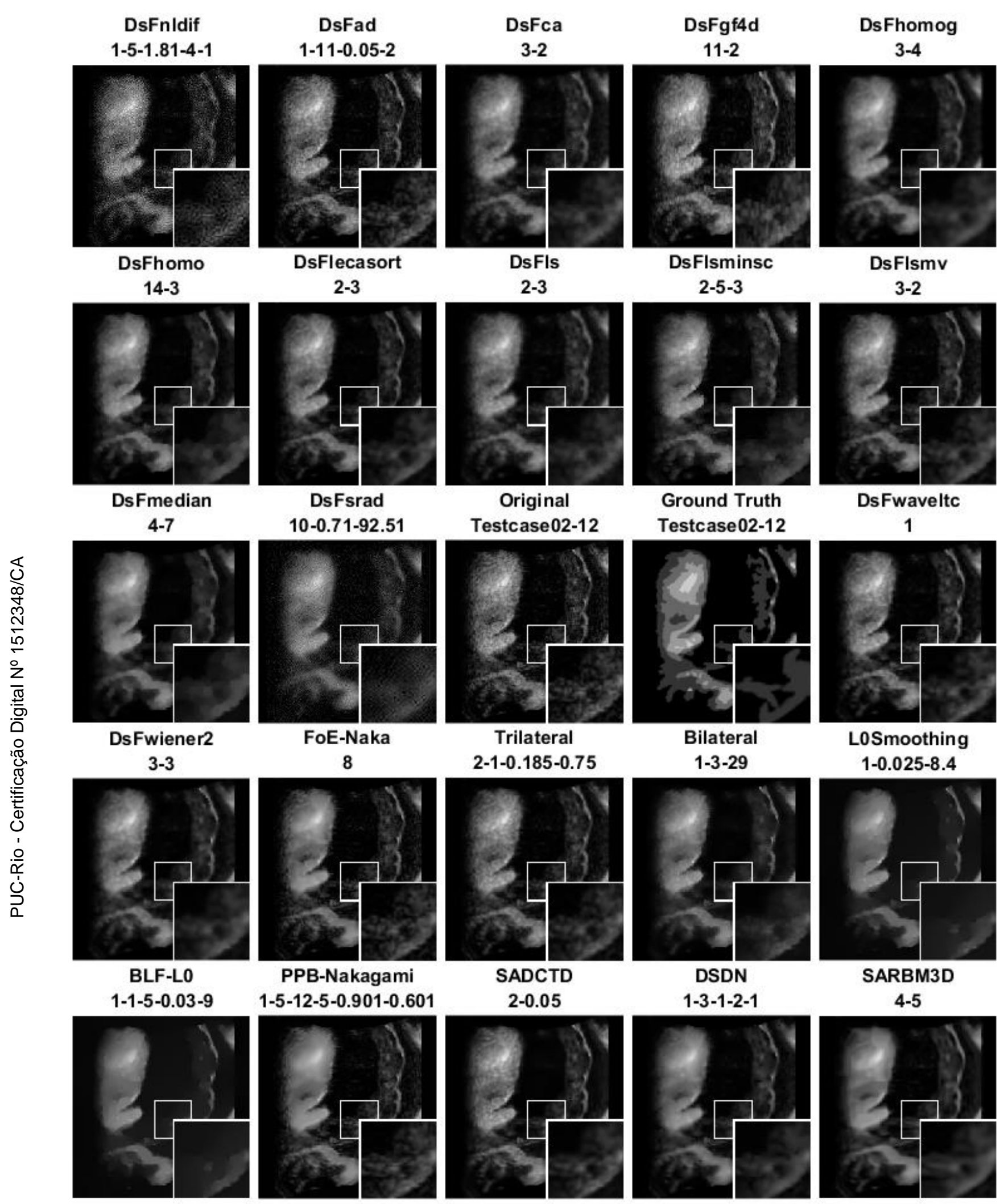

Figura 8.4: Aplicações de todos os filtros discutidos no capítulo 3, com as configurações de parâmetros determinadas na seção 6.9 na imagem de teste Testcase 02-12. No canto inferior direito de cada imagem encontra-se uma região ampliada de uma área de interesse do exame que está sendo analisado. 


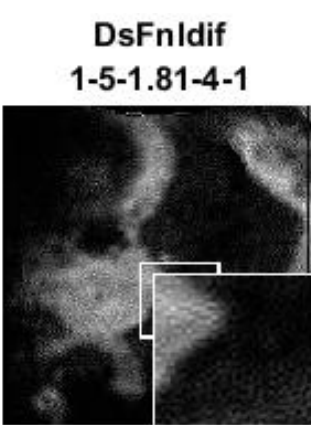

DsFhomo

14-3

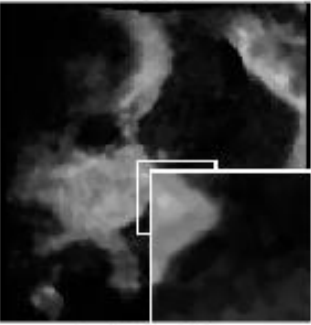

DsFmedian

4-7

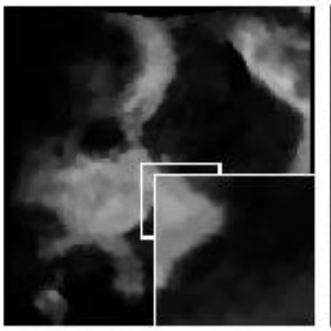

DsFwiener2

3-3

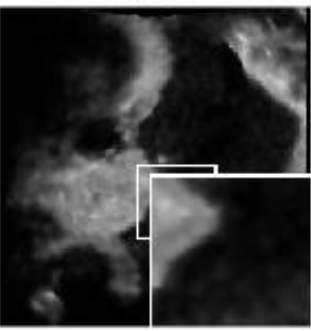

BLF-LO

1-1-5-0.03-9

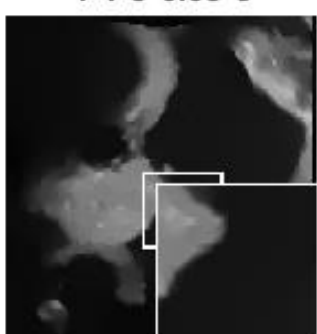

DsFad

1-11-0.05-2

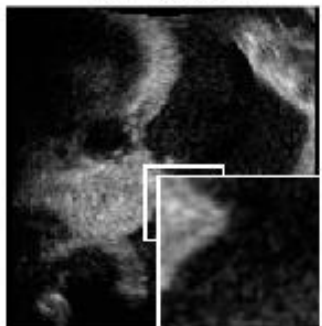

DsFlecasort

2-3

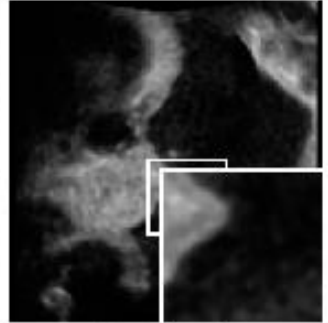

DsFsrad

10-0.71-92.51

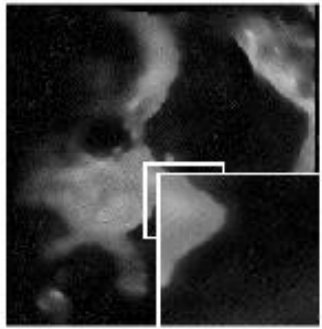

FoE-Naka

8

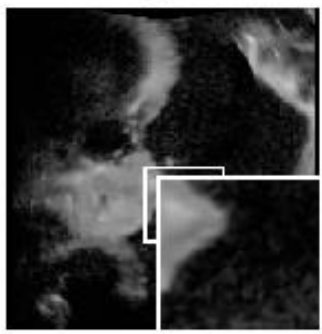

PPB-Nakagami 1-5-12-5-0.901-0.601

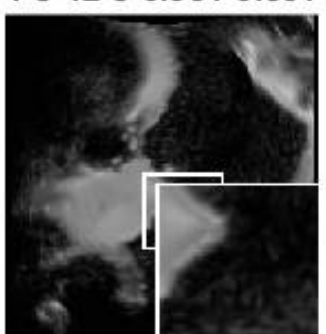

DsFca

3-2

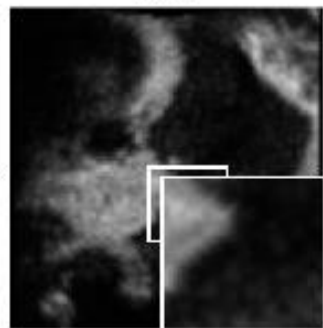

DsFls

2-3

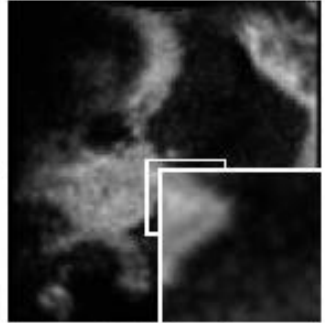

Original

Testcase 02-29

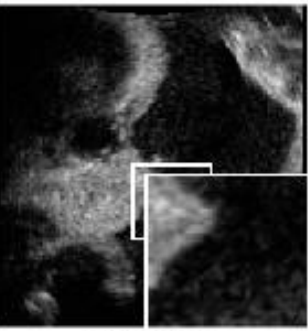

Trilateral

2-1-0.185-0.75

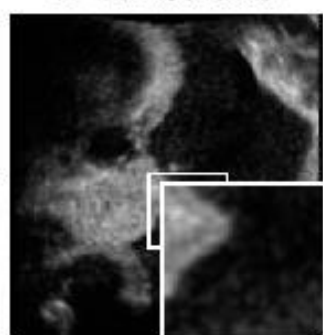

SADCTD

2-0.05

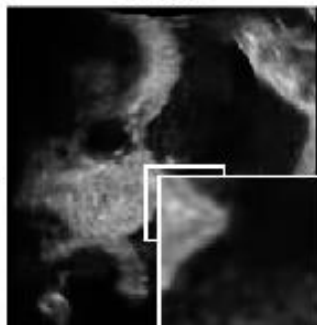

DsFgf4d

11-2

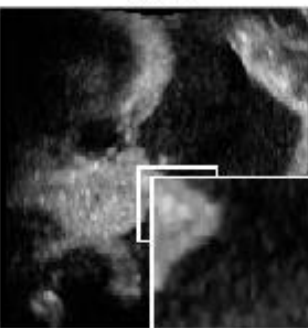

DsFlsminsc

2-5-3

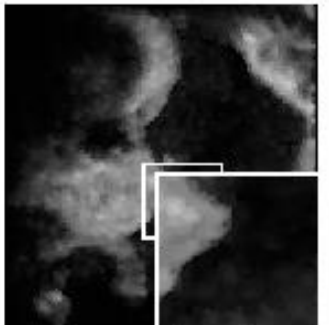

Ground Truth

Testcase 02-29

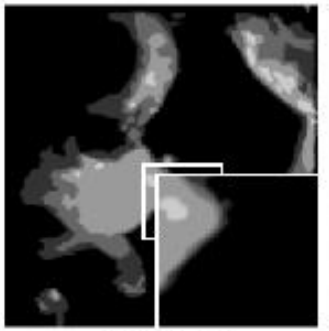

Bilateral

1-3-29

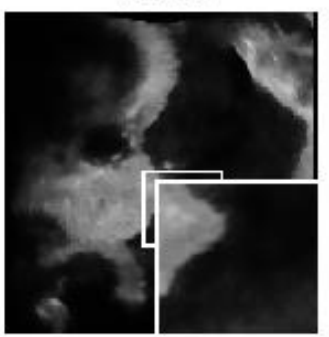

DSDN

1-3-1-2-1

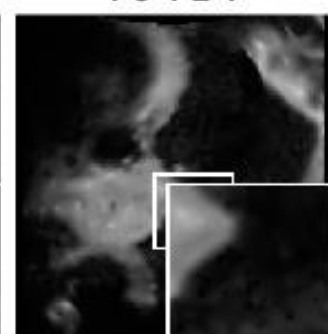

DsFhomog

3-4

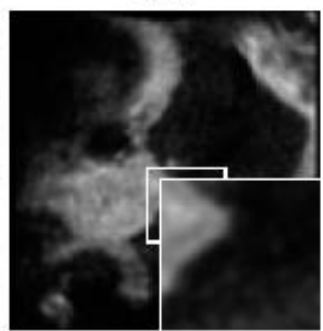

DsFIsmv 3-2

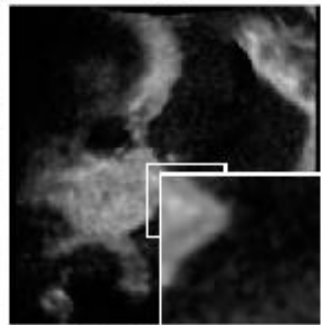

DsFwaveltc

1

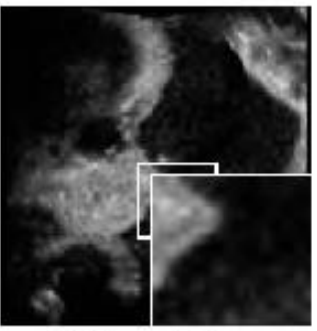

LOSmoothing 1-0.025-8.4

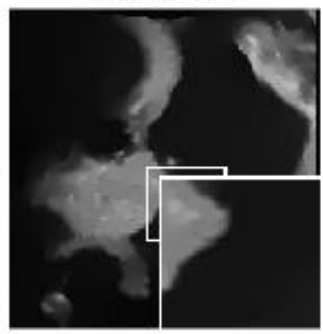

SARBM3D

4-5

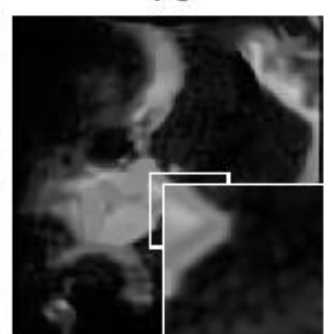

Figura 8.5: Aplicações de todos os filtros discutidos no capítulo 3, com as configurações de parâmetros determinadas na seção 6.9 na imagem de teste Testcase 02-29. No canto inferior direito de cada imagem encontra-se uma região ampliada de uma área de interesse do exame que está sendo analisado. 


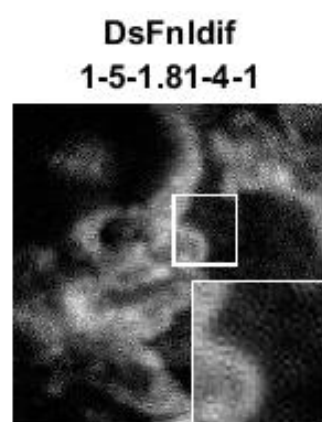

DsFhomo

14-3

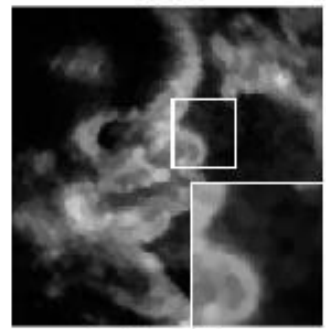

DsFmedian

4-7

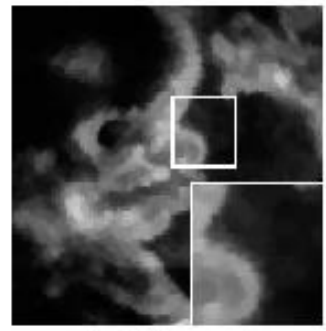

DsFwiener2

3-3

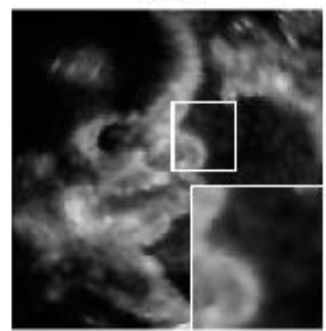

BLF-LO

1-1-5-0.03-9

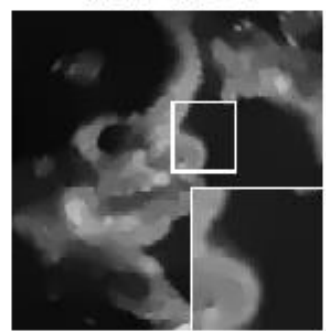

DsFad

1-11-0.05-2

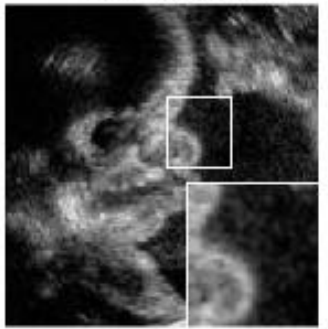

DsFlecasort

2-3

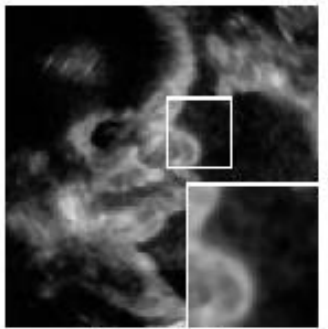

DsFsrad

10-0.71-92.51

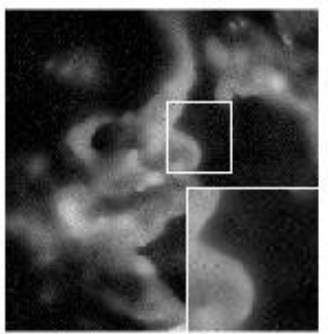

FoE-Naka

8

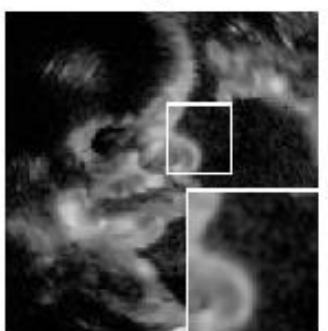

PPB-Nakagami 1-5-12-5-0.901-0.601

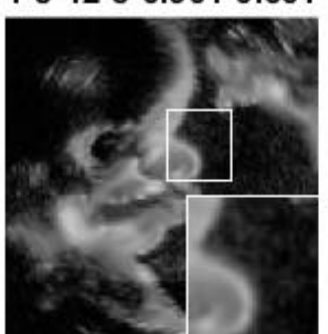

DsFca

3-2

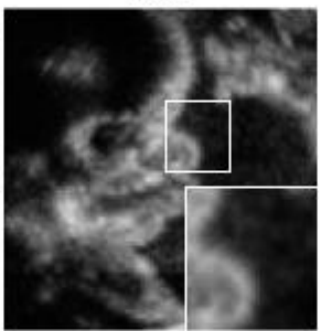

DsFls

2-3

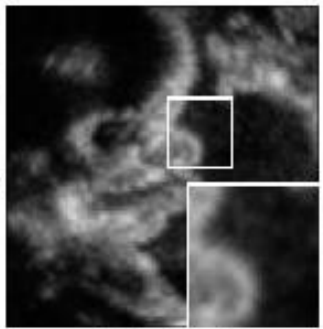

Original

Testcase 02-45

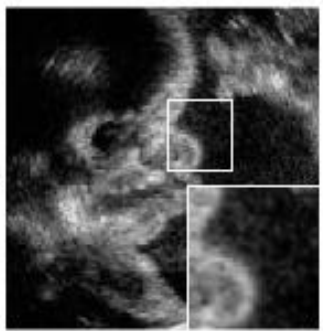

Trilateral

2-1-0.185-0.75

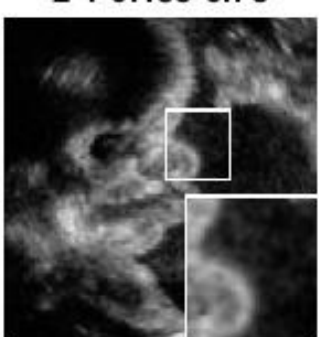

SADCTD

2-0.05

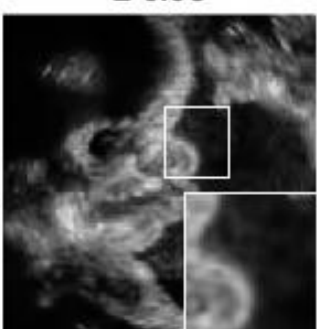

DsFgf4d

11-2

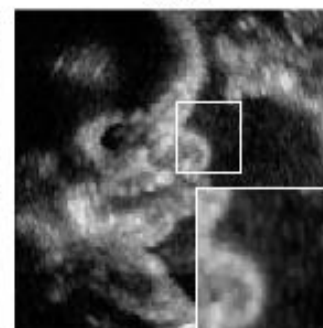

DsFlsminsc

2-5-3

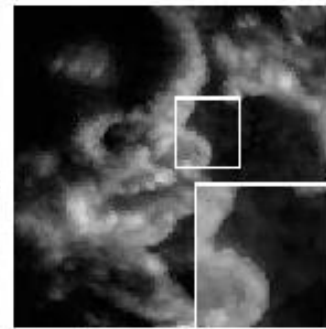

Ground Truth

Testcase 02-45

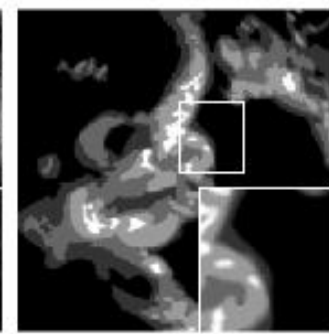

Bilateral

1-3-29

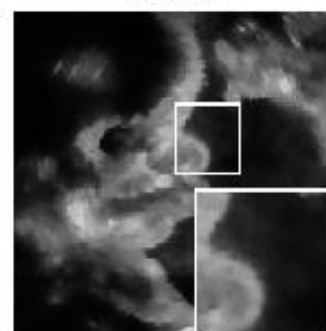

DSDN

1-3-1-2-1

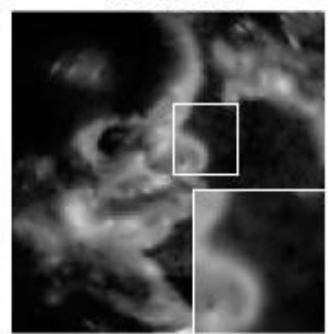

DsFhomog

3-4

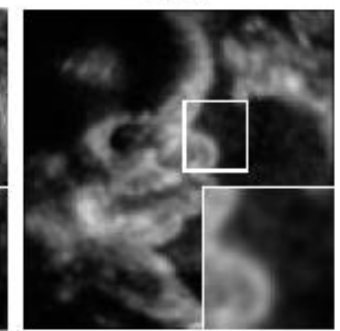

DsFlsmv

3-2

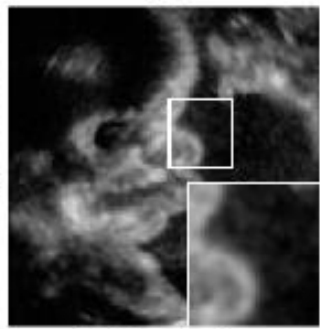

DsFwaveltc

1

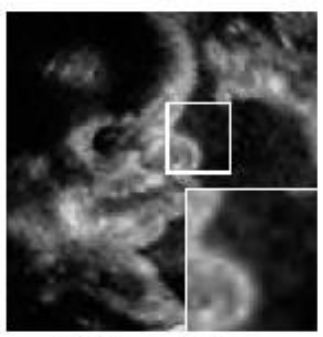

LOSmoothing

1-0.025-8.4

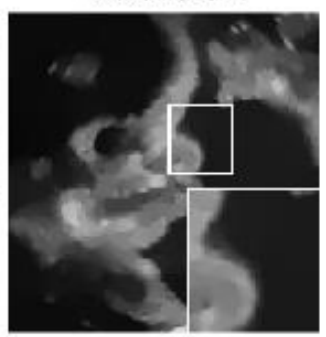

SARBM3D

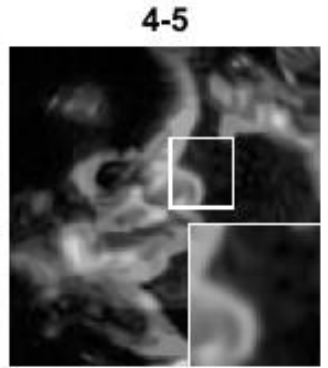

Figura 8.6: Aplicações de todos os filtros discutidos no capítulo 3, com as configurações de parâmetros determinadas na seção 6.9 na imagem de teste Testcase 02-45. No canto inferior direito de cada imagem encontra-se uma região ampliada de uma área de interesse do exame que está sendo analisado. 

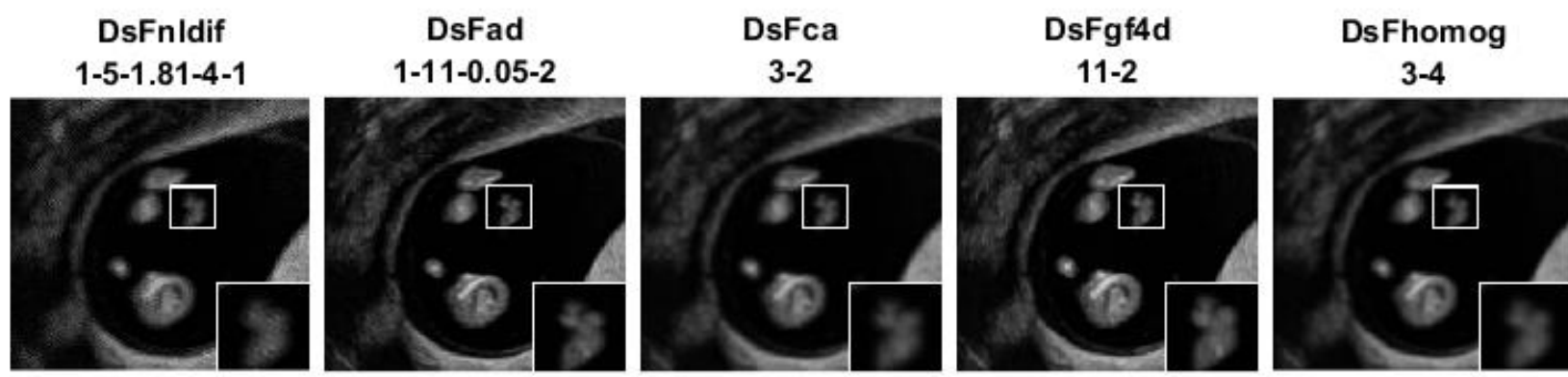
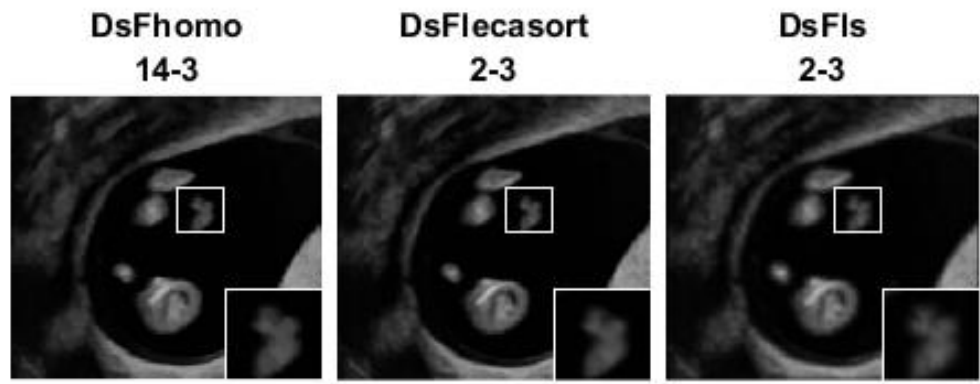

DsFlsminsc

2-5-3

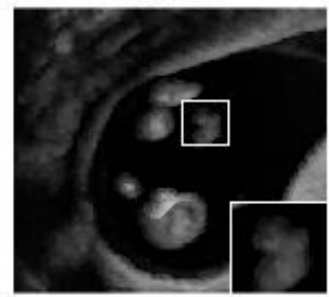

Ground Truth Testcase 03-29

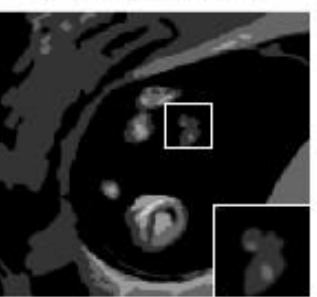

Bilateral 1-3-29

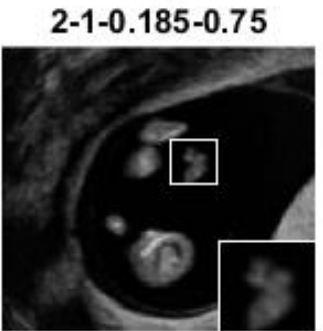

SADCTD

2-0.05

1-5-12-5-0.901-0.601
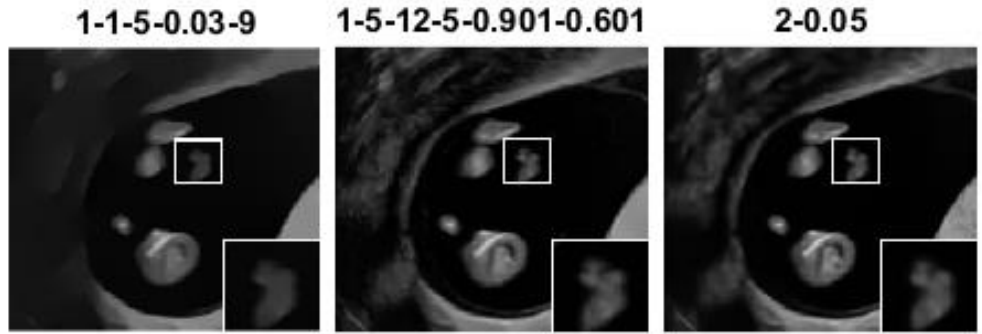

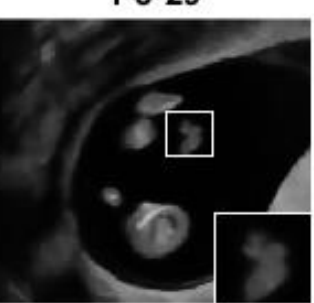

DSDN

1-3-1-2-1

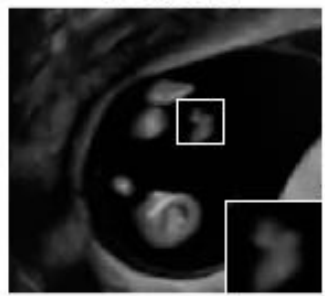

DsFIsmv

3-2

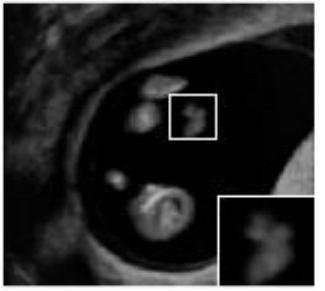

DsFwaveltc

1

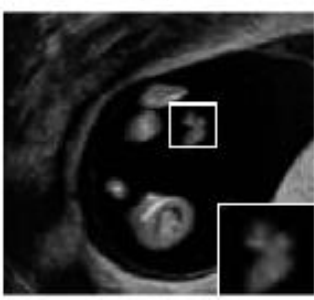

L0Smoothing

1-0.025-8.4

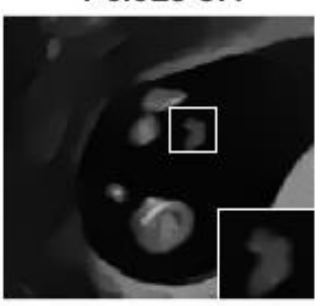

SARBM3D

4-5

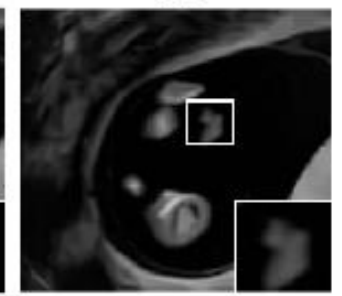

Figura 8.7: Aplicações de todos os filtros discutidos no capítulo 3, com as configurações de parâmetros determinadas na seção 6.9 na imagem de teste Testcase 03-29. No canto inferior direito de cada imagem encontra-se uma região ampliada de uma área de interesse do exame que está sendo analisado. 

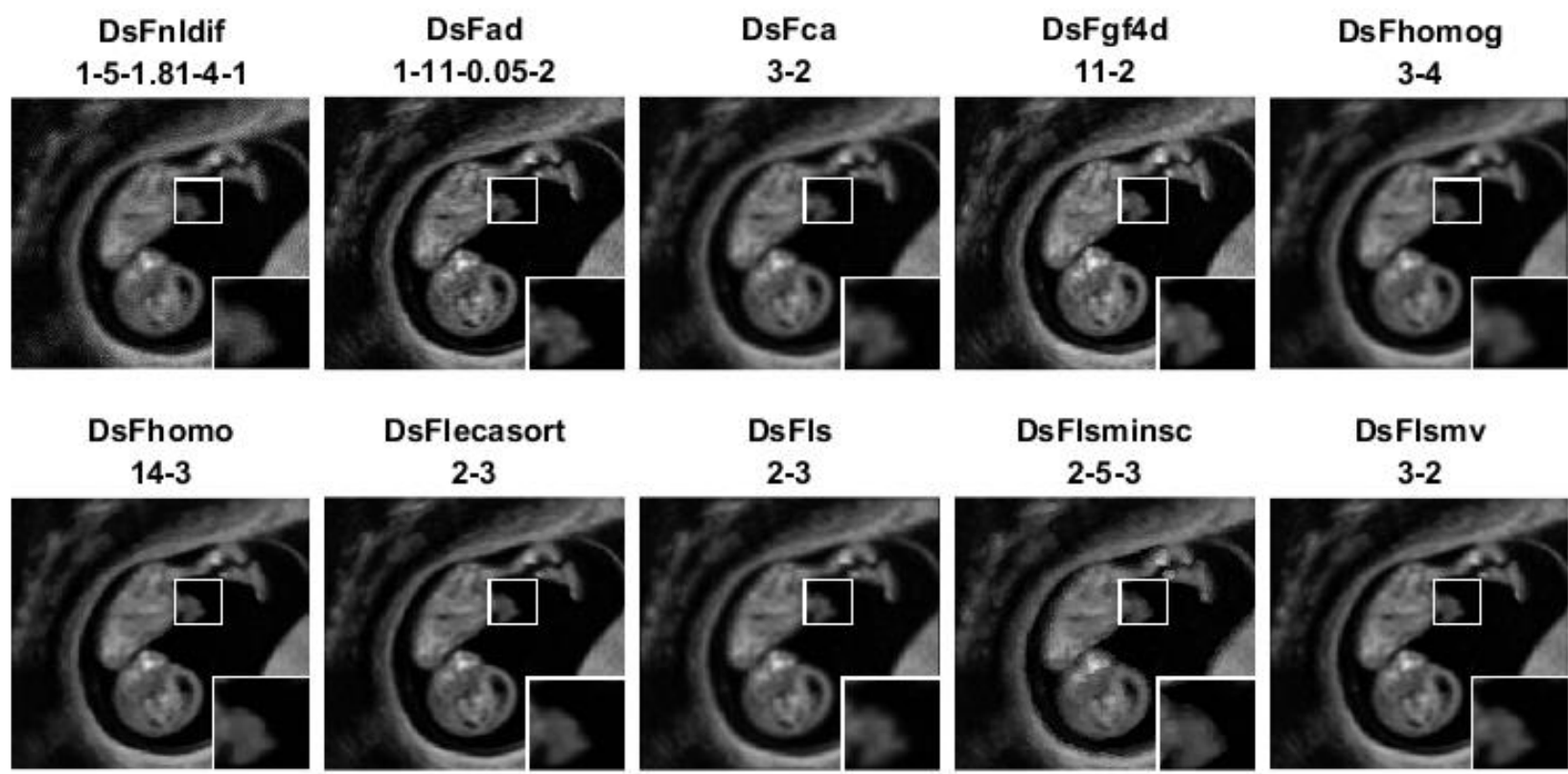

DsFlsmv

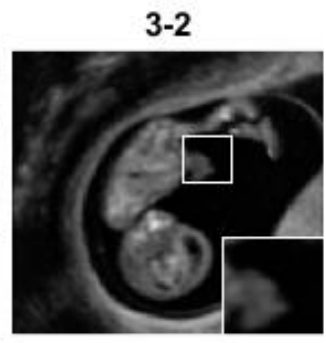

DsFmedian

DsFsrad

Original

10-0.71-92.51

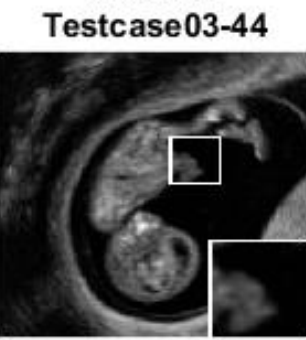

Ground Truth

DsFwaveltc
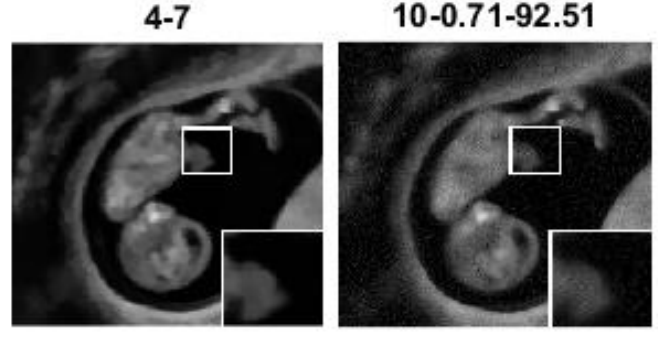

Testcase 03-44

1
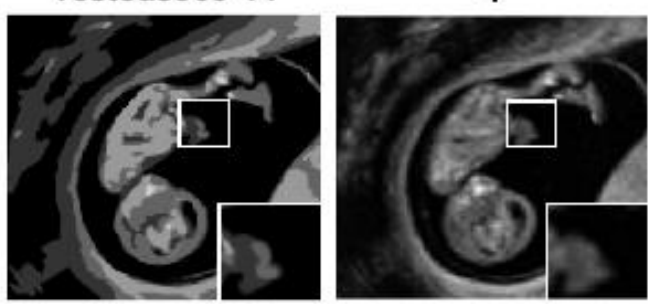

\section{DsFwiener2}

3-3

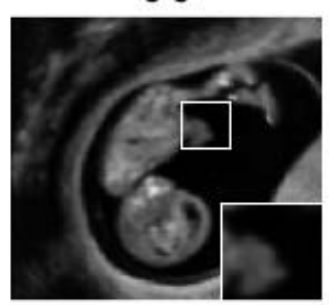

FoE-Naka

8

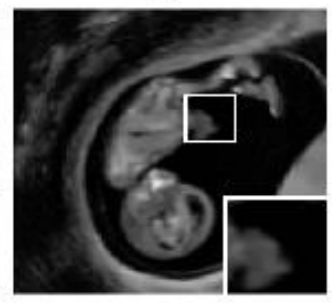

Trilateral

2-1-0.185-0.75

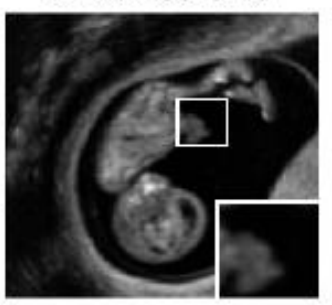

Bilateral

1-3-29

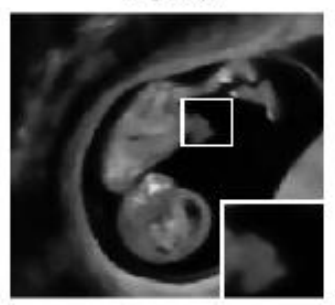

LOSmoothing

1-0.025-8.4

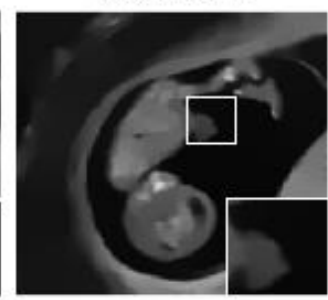

BLF-LO

PPB-Nakagami

SADCTD

2-0.05

DSDN

1-3-1-2-1
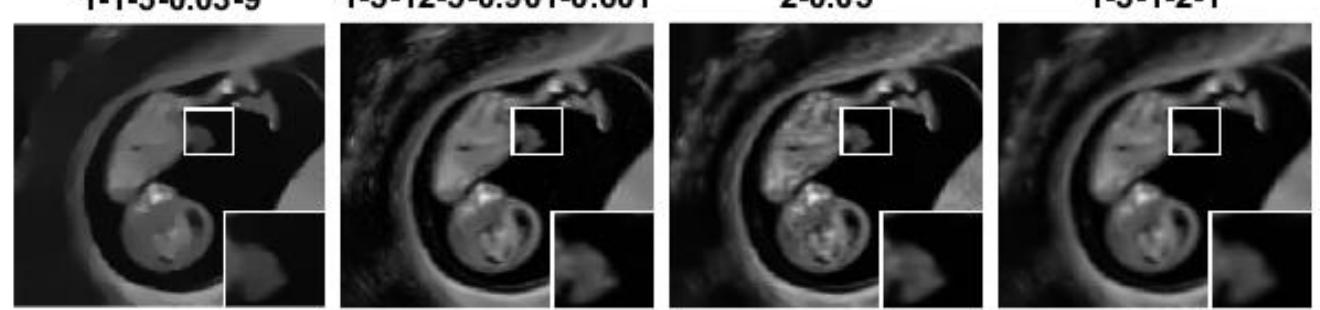

SARBM3D

4-5

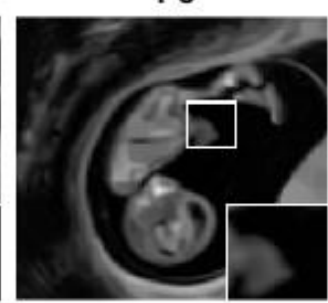

Figura 8.8: Aplicações de todos os filtros discutidos no capítulo 3, com as configurações de parâmetros determinadas na seção 6.9 na imagem de teste Testcase 03-44. No canto inferior direito de cada imagem encontra-se uma região ampliada de uma área de interesse do exame que está sendo analisado. 

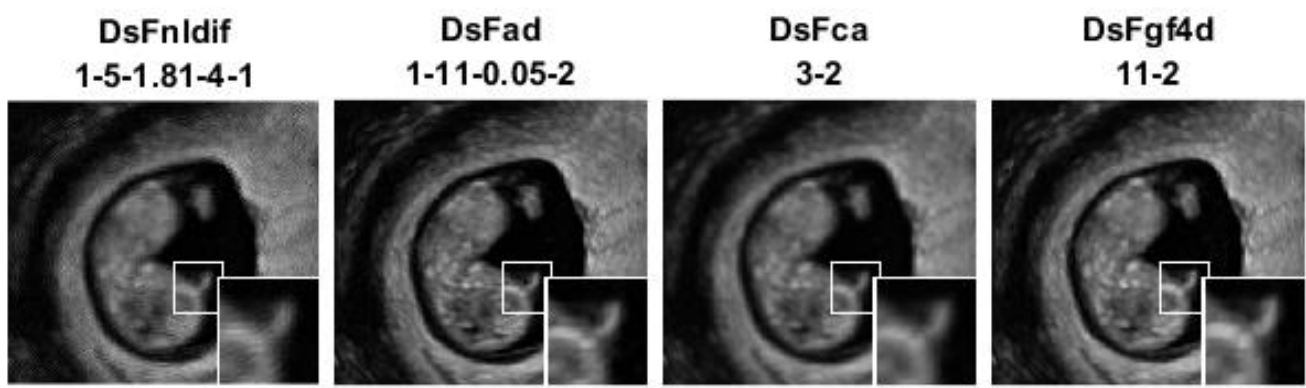

DsFhomog

3-4

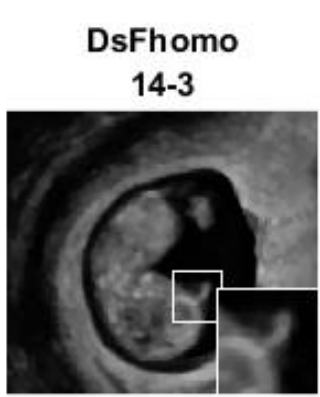

\section{DsFlecasort}

2-3

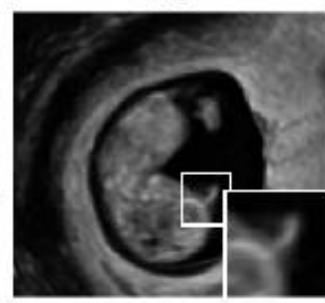

DsFls

2-3

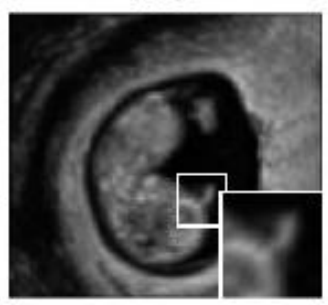

DsFlsminsc

2-5-3
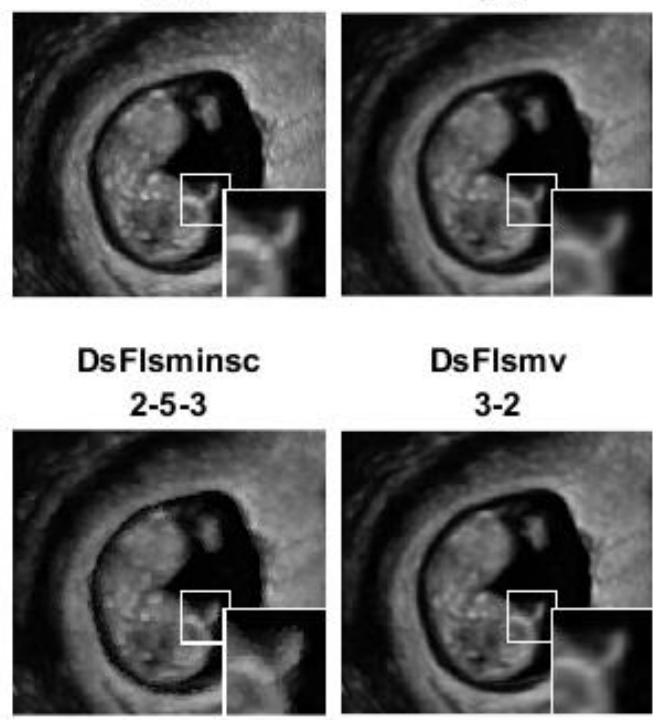

DsFmedian
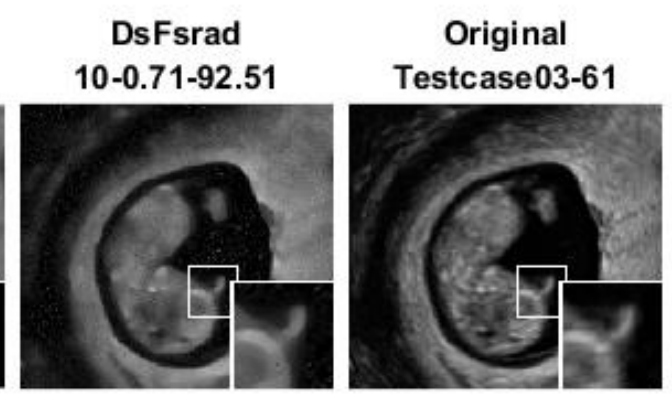

Ground Truth

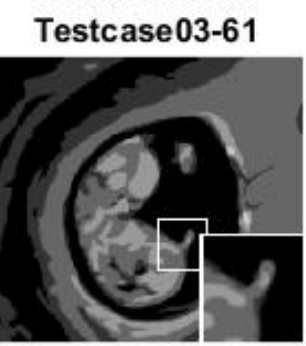

DsFwaveltc

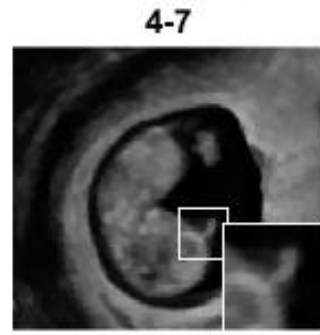

FoE-Naka

8
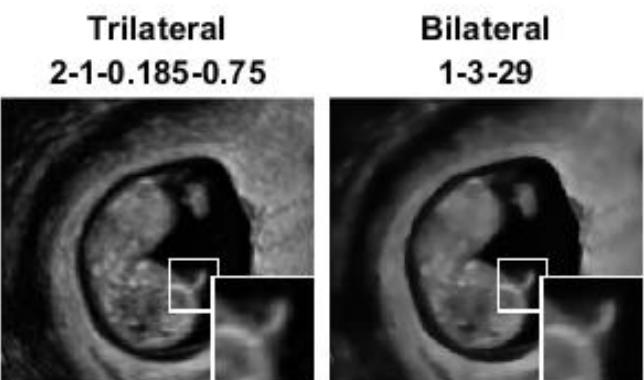

LOSmoothing

3-3
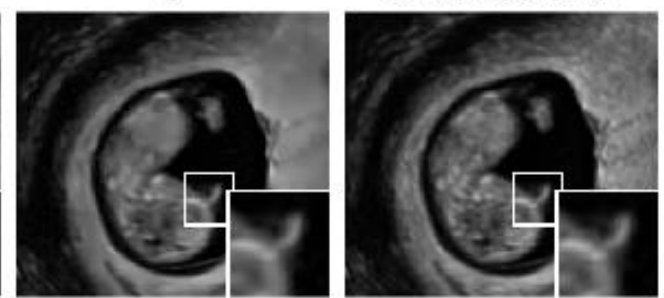

PPB-Nakagami 1-5-12-5-0.901-0.601

SADCTD

BLF-LO

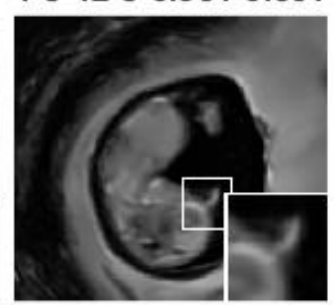

2-0.05

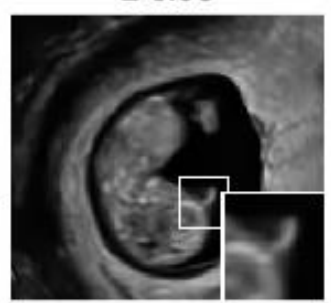

DSDN

1-3-1-2-1
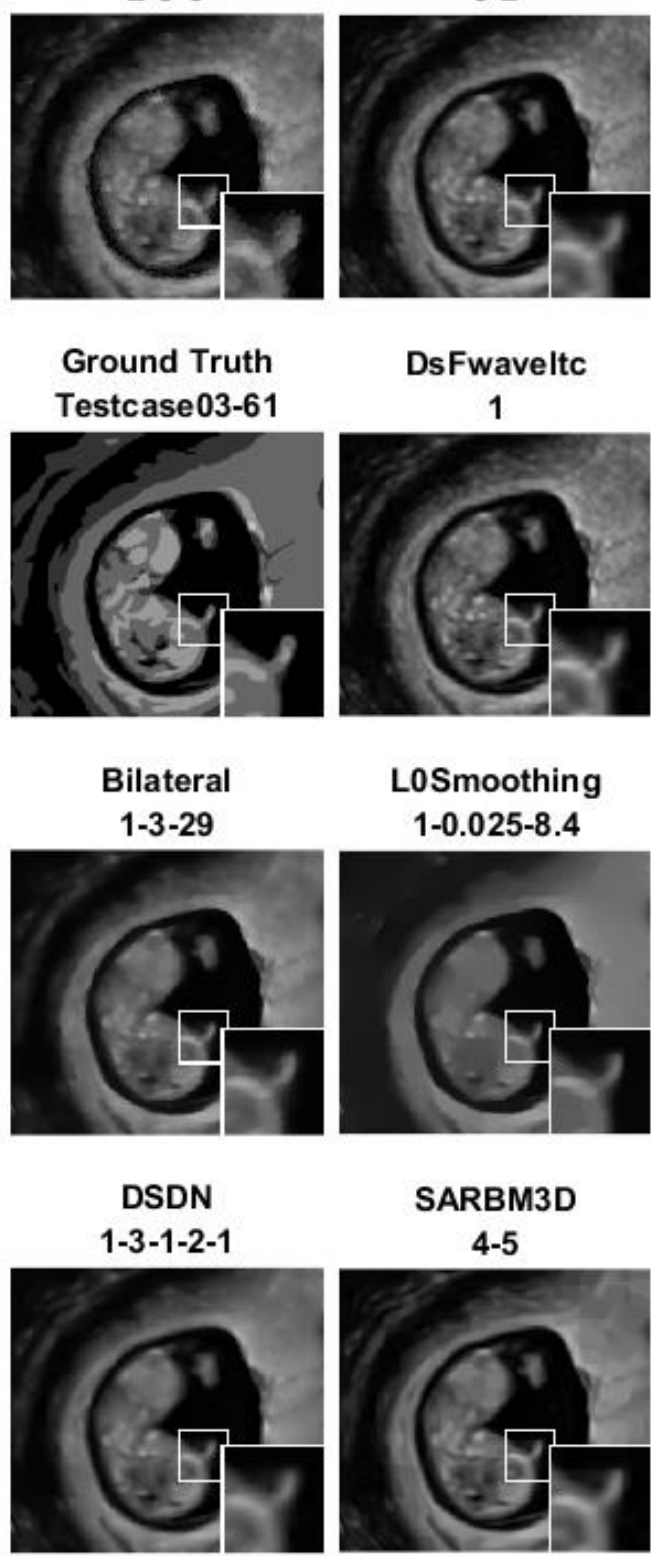

DsFIsmv

3-2

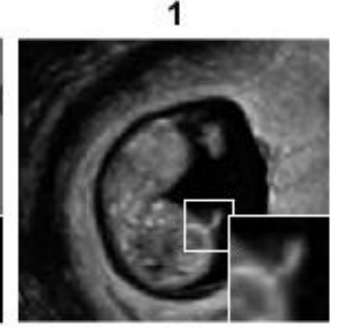

1-0.025-8.4

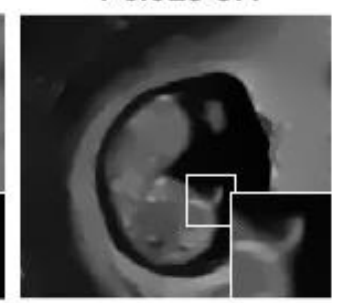

SARBM3D

4-5

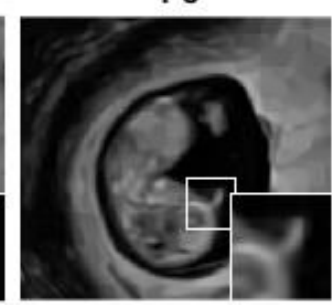

Figura 8.9: Aplicações de todos os filtros discutidos no capítulo 3, com as configurações de parâmetros determinadas na seção 6.9 na imagem de teste Testcase 03-61. No canto inferior direito de cada imagem encontra-se uma região ampliada de uma área de interesse do exame que está sendo analisado. 


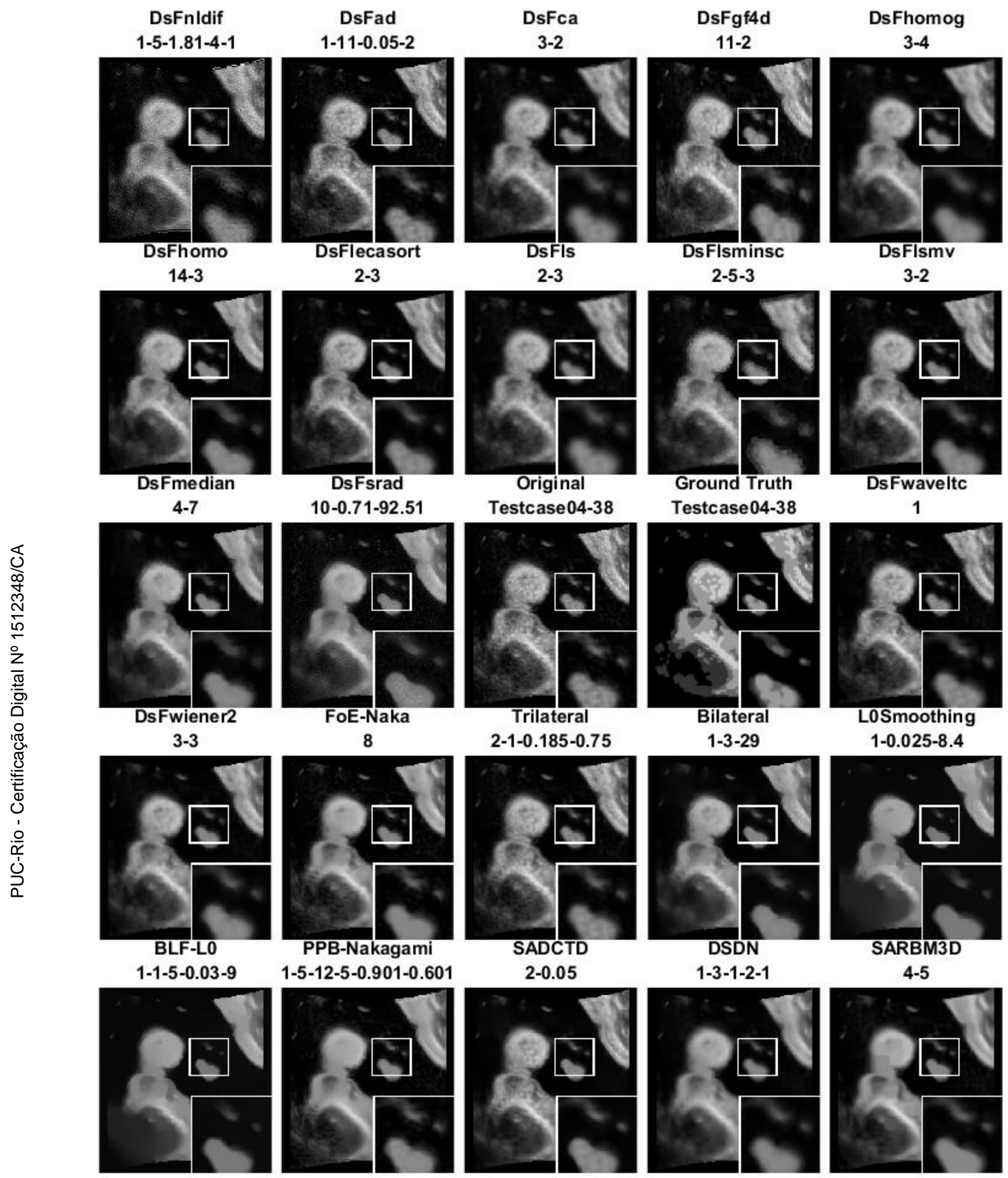

Figura 8.10: Aplicações de todos os filtros discutidos no capítulo 3, com as configurações de parâmetros determinadas na seção 6.9 na imagem de teste Testcase 04-38. No canto inferior direito de cada imagem encontra-se uma região ampliada de uma área de interesse do exame que está sendo analisado. 

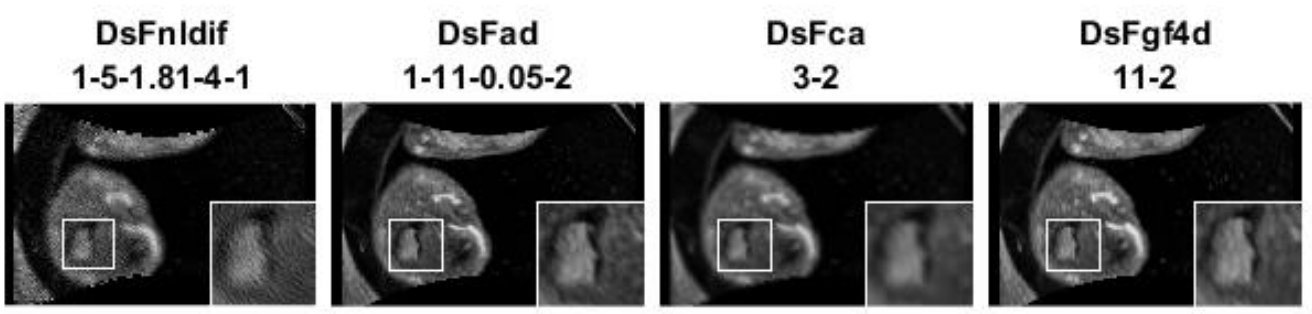

DsFhomog

3-4

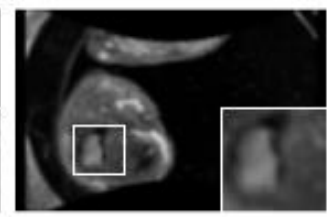

DsFhomo

14-3

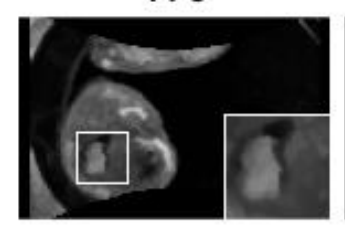

DsFlecasort

2-3

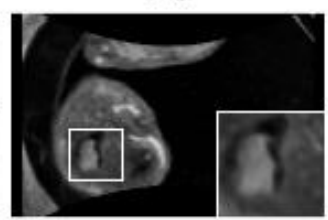

DsFls

2-3

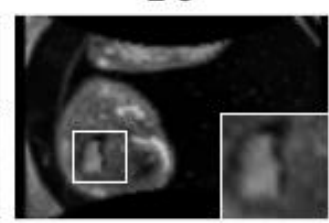

DsFlsminsc

2-5-3

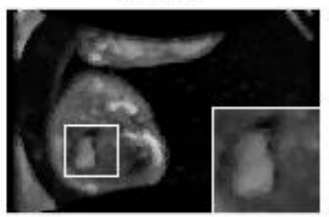

DsFlsmv

3-2

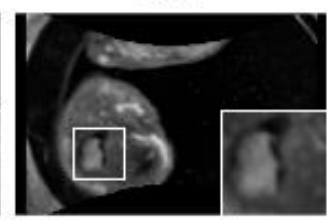

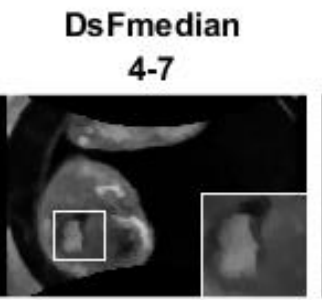
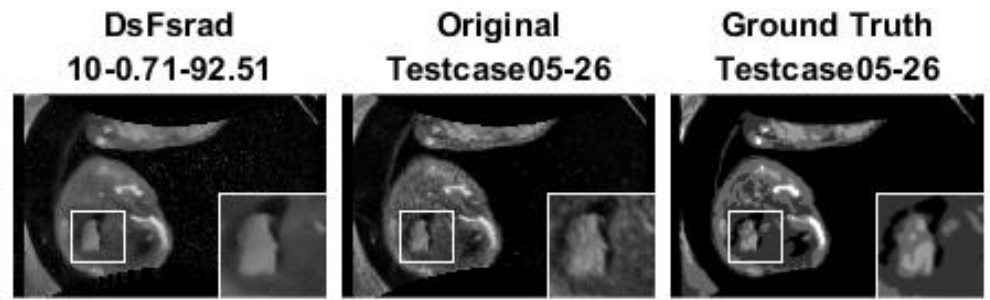

DsFwaveltc

1
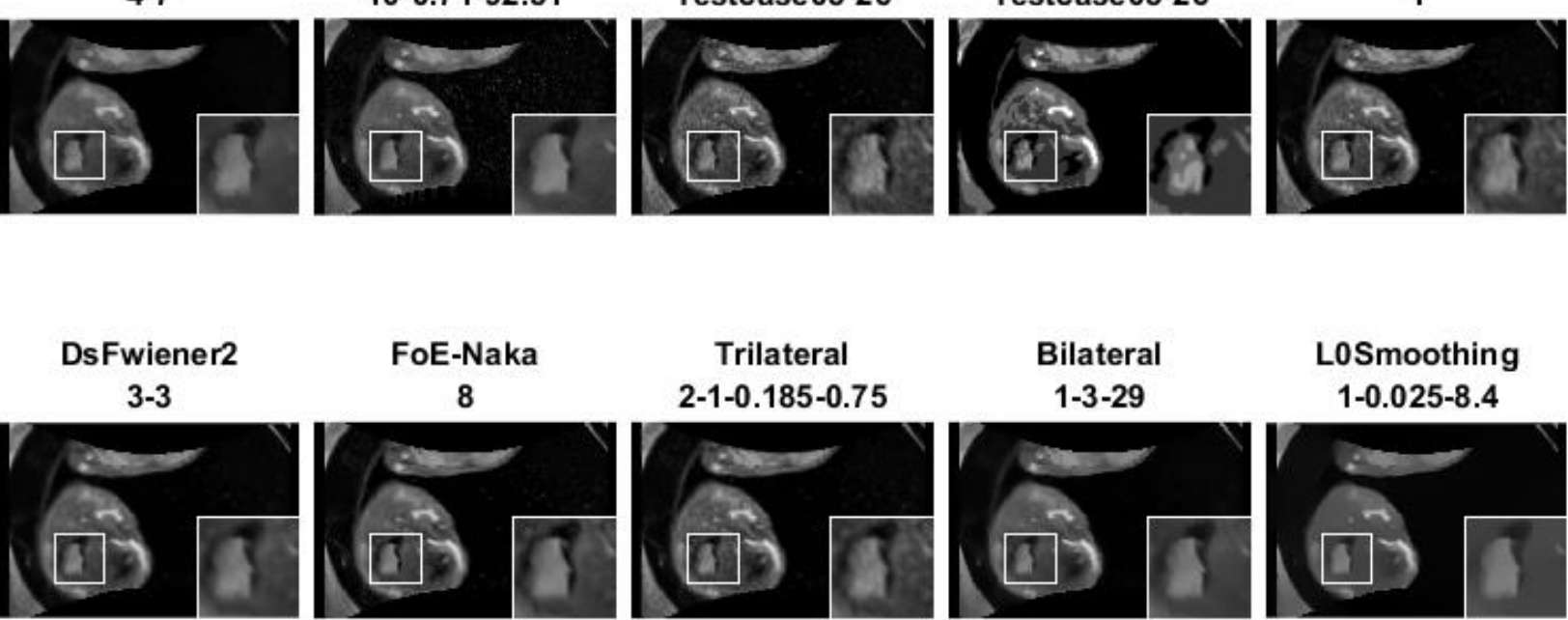

LOSmoothing 1-0.025-8.4
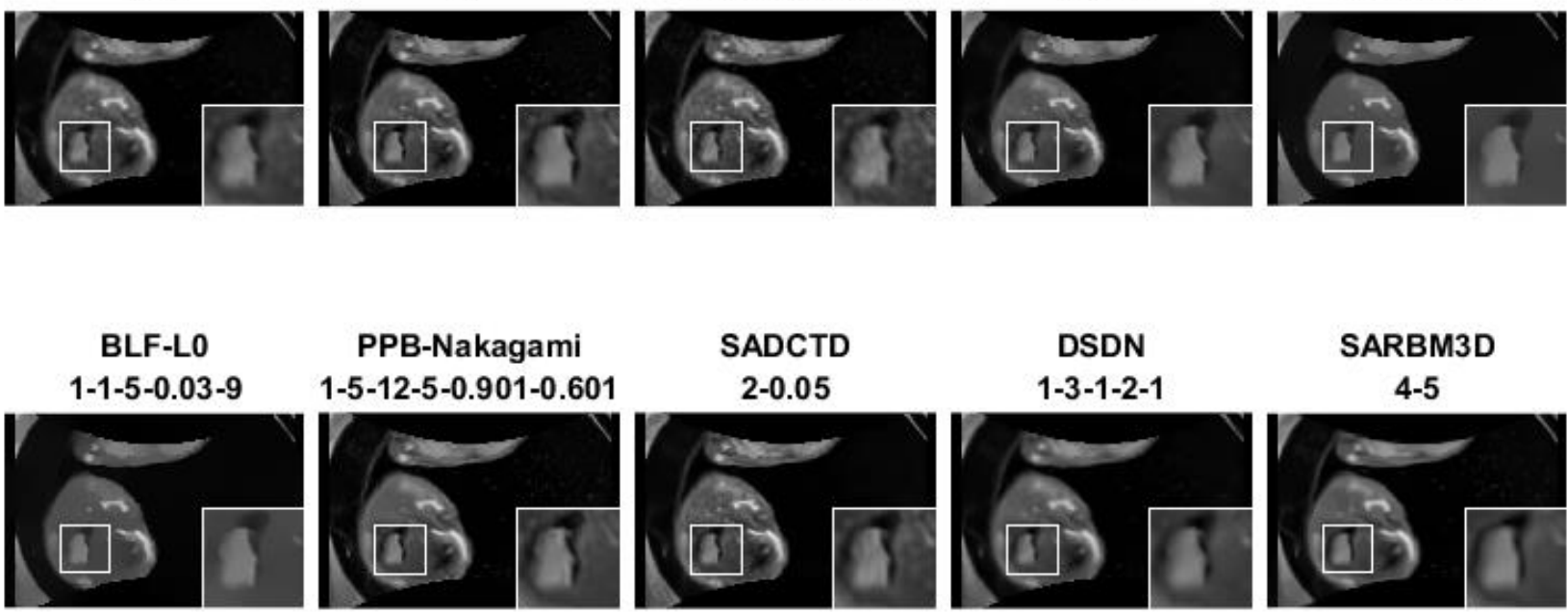

PPB-Nakagami 1-5-12-5-0.901-0.601

SADCTD

2-0.05

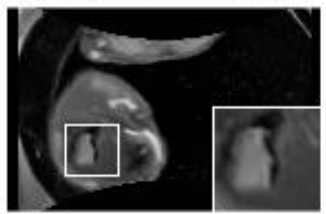

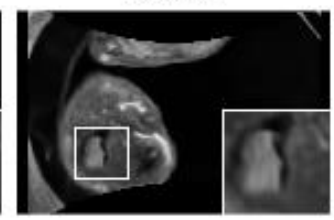

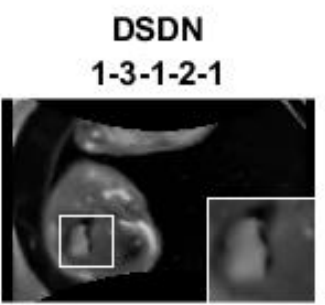

SARBM3D

4-5

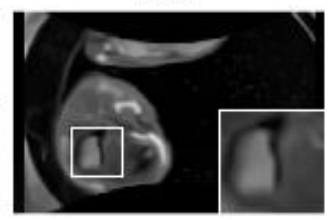

Figura 8.11: Aplicações de todos os filtros discutidos no capítulo 3, com as configurações de parâmetros determinadas na seção 6.9 na imagem de teste Testcase 05-26. No canto inferior direito de cada imagem encontra-se uma região ampliada de uma área de interesse do exame que está sendo analisado. 


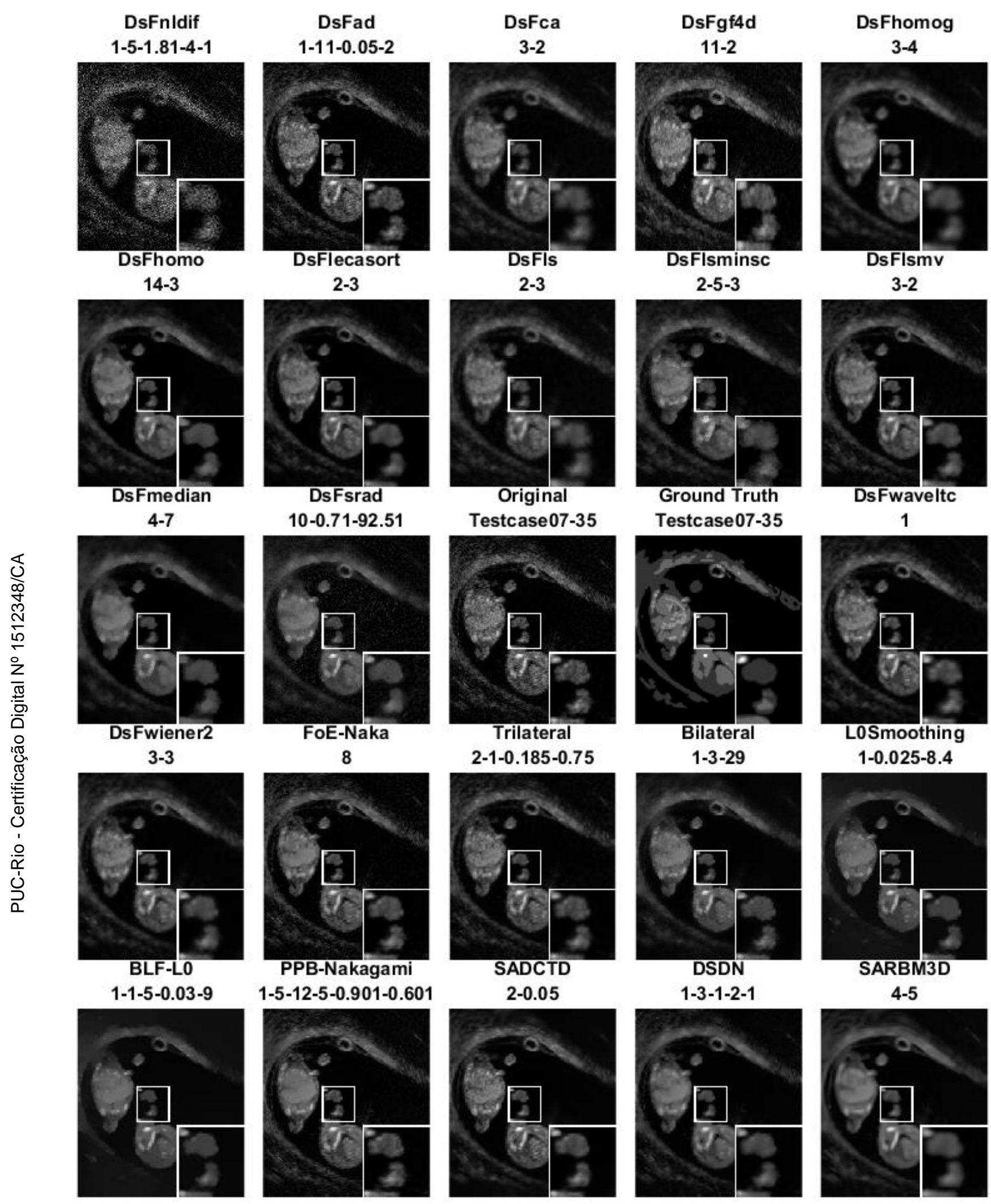

Figura 8.12: Aplicações de todos os filtros discutidos no capítulo 3, com as configurações de parâmetros determinadas na seção 6.9 na imagem de teste Testcase 07-35. No canto inferior direito de cada imagem encontra-se uma região ampliada de uma área de interesse do exame que está sendo analisado. 


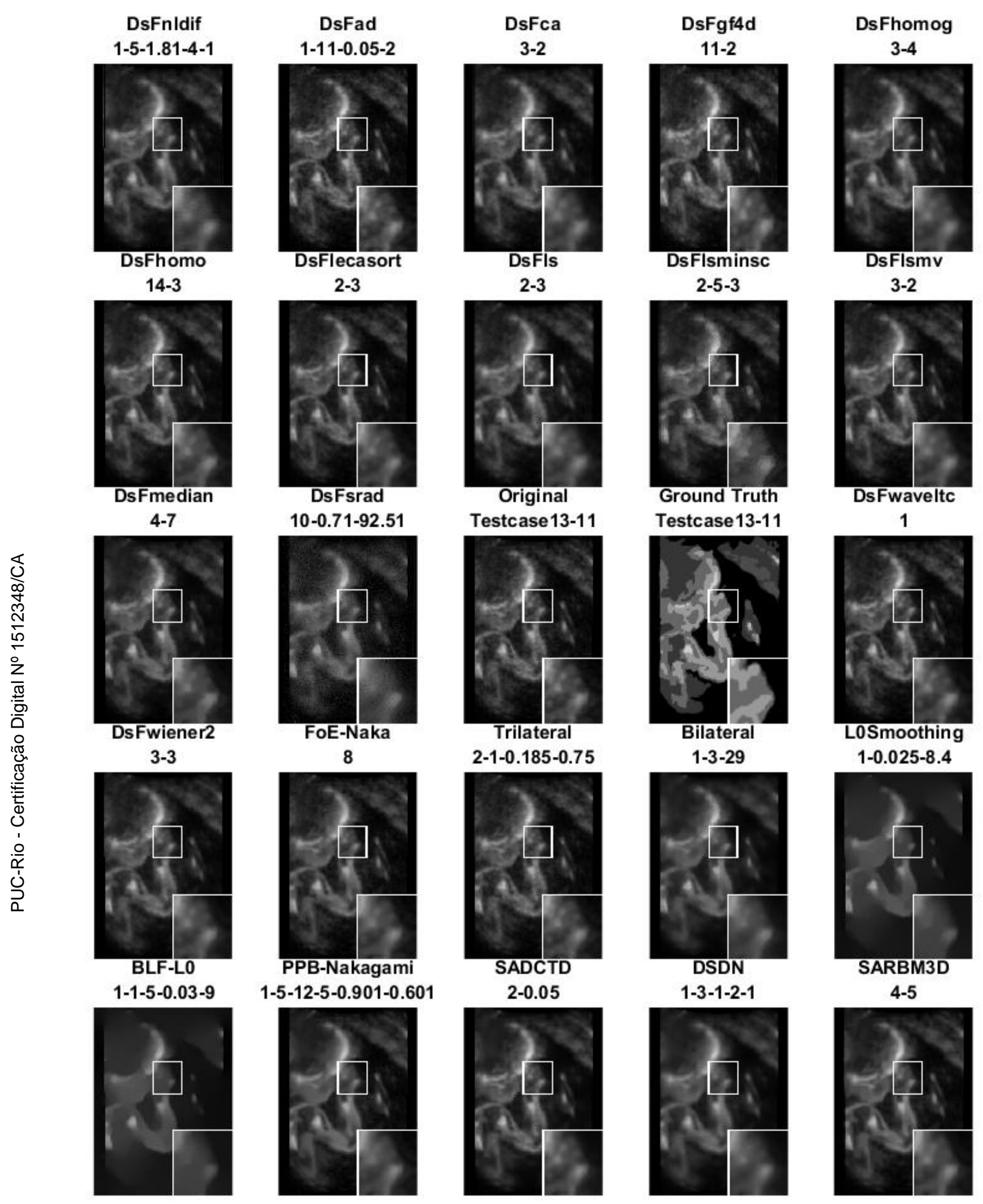

Figura 8.13: Aplicações de todos os filtros discutidos no capítulo 3, com as configurações de parâmetros determinadas na seção 6.9 na imagem de teste Testcase 13-11. No canto inferior direito de cada imagem encontra-se uma região ampliada de uma área de interesse do exame que está sendo analisado. 


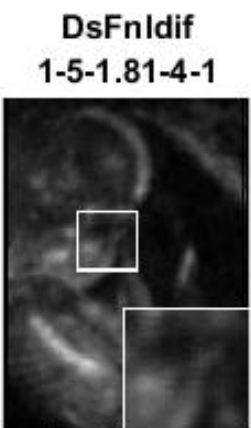

DsFhomo

14-3

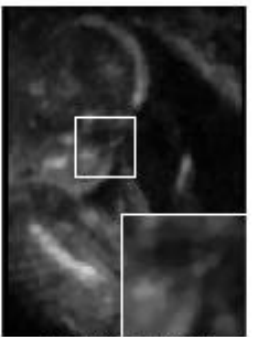

DsFmedian

4-7

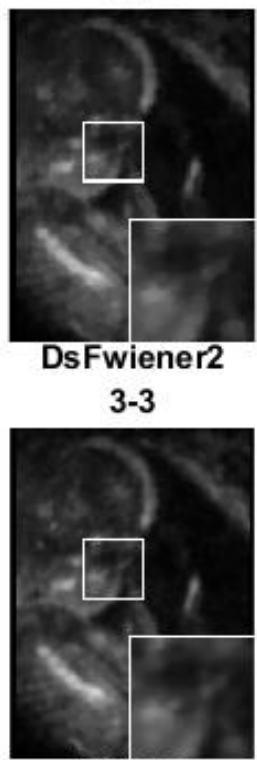

BLF-L0

1-1-5-0.03-9

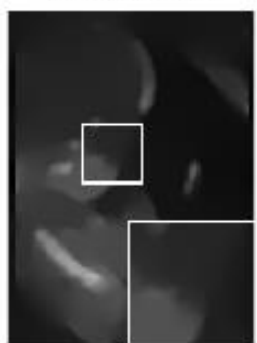

DsFad

1-11-0.05-2

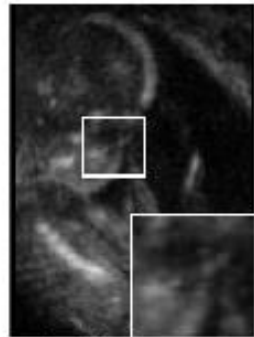

DsFlecasort

2-3

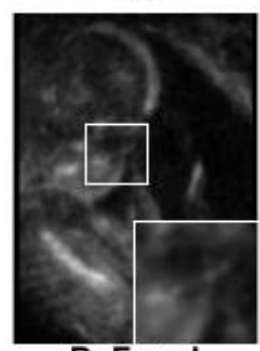

DsFsrad
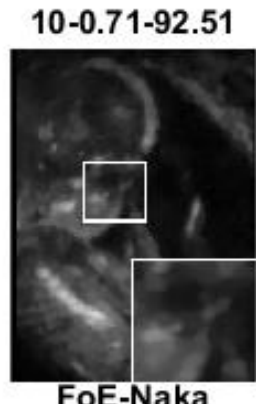

8

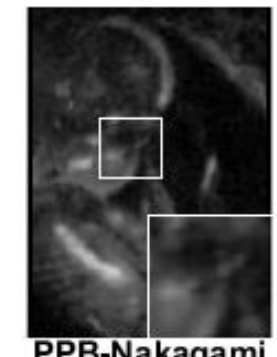

PPB-Nakagami 1-5-12-5-0.901-0.601

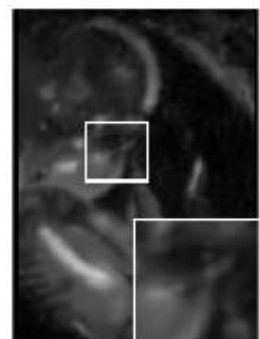

DsFca

3-2

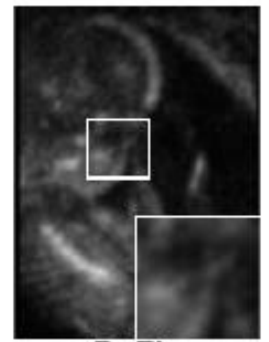

DsFls

2-3

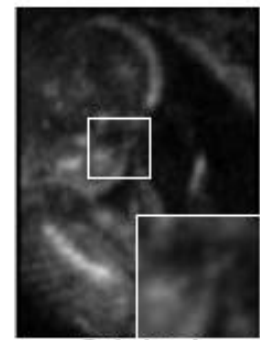

Original

Testcase 13-28

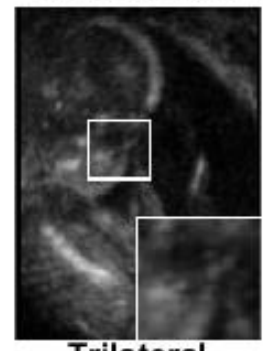

Trilateral

2-1-0.185-0.75

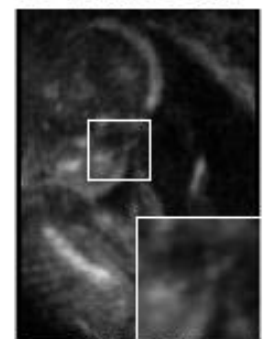

SADCTD

2-0.05

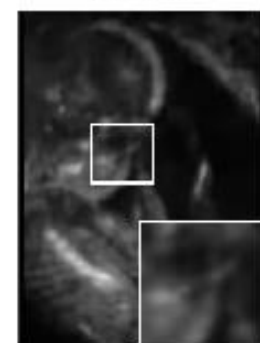

DsFgf4d

11-2

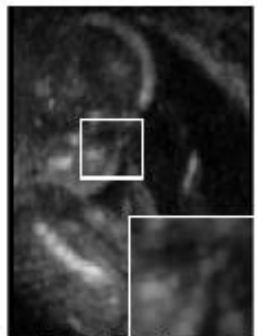

DsFlsminsc

2-5-3

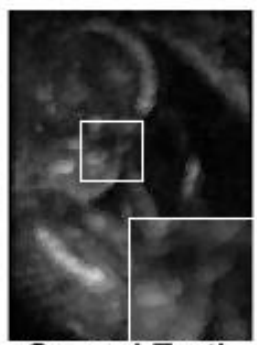

Ground Truth

Testcase13-28

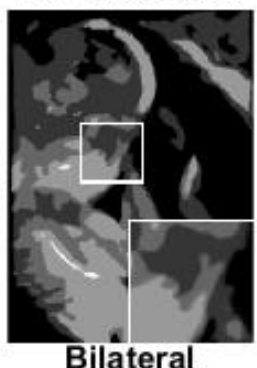

Bilateral

1-3-29

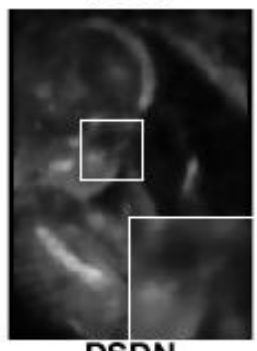

DSDN

1-3-1-2-1

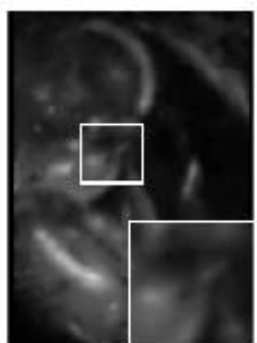

DsFhomog

3-4

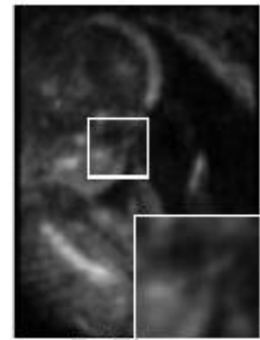

DsFlsmv

3-2

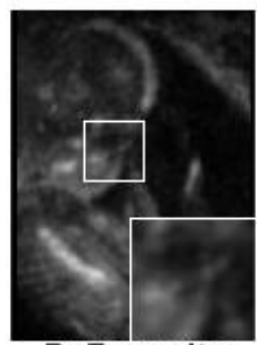

DsFwaveltc

1

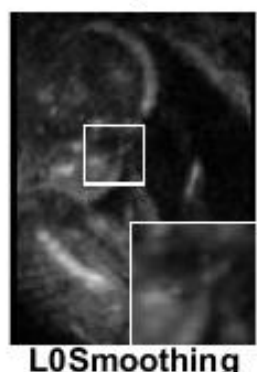

1-0.025-8.4

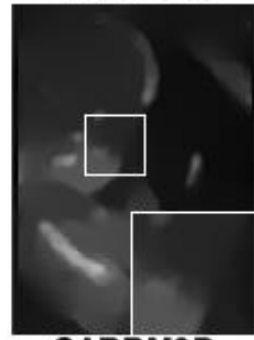

SARBM3D

4-5

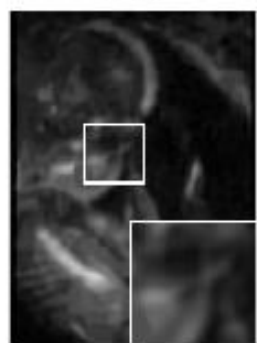

Figura 8.14: Aplicações de todos os filtros discutidos no capítulo 3, com as configurações de parâmetros determinadas na seção 6.9 na imagem de teste Testcase 13-28. No canto inferior direito de cada imagem encontra-se uma região ampliada de uma área de interesse do exame que está sendo analisado. 


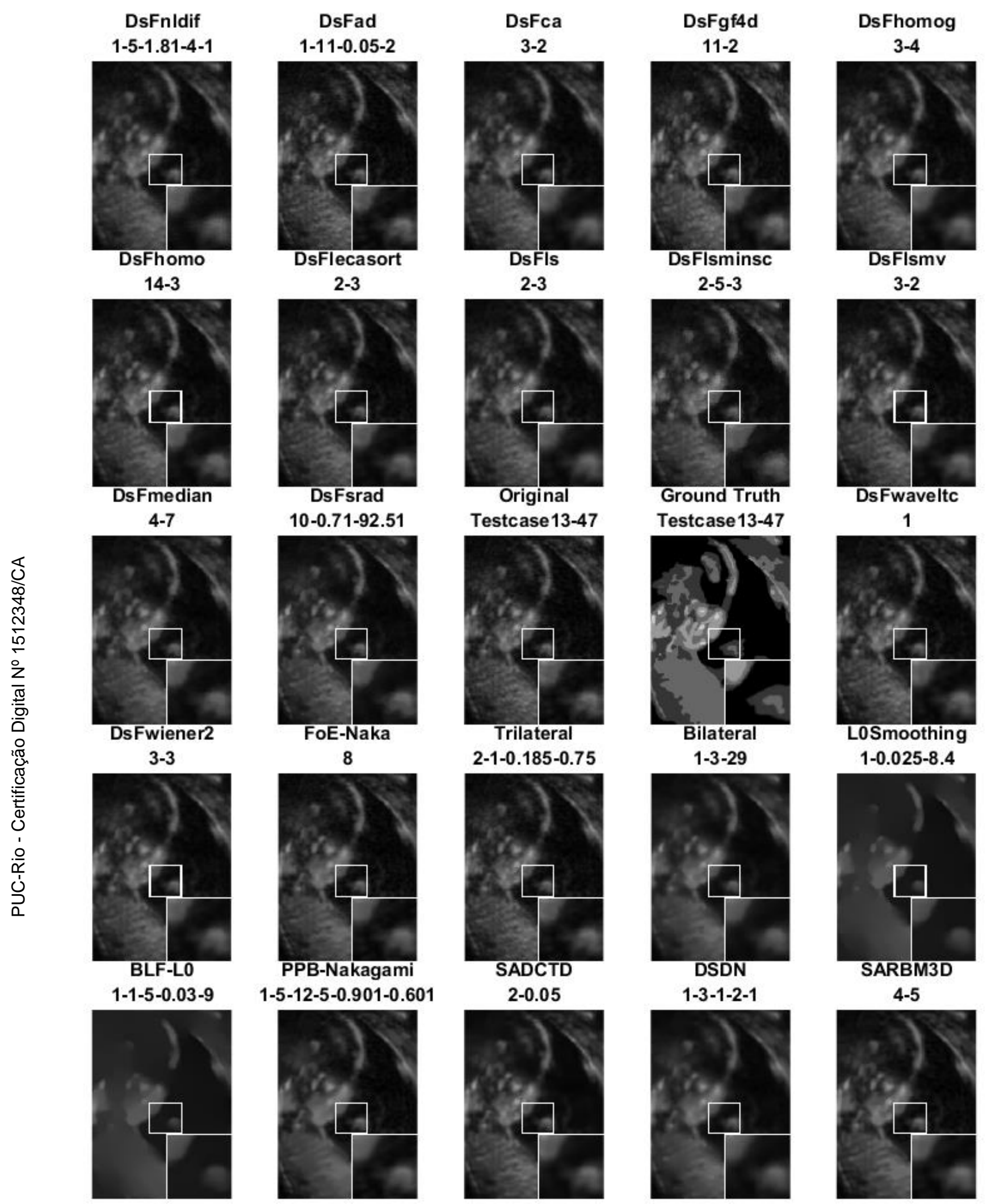

Figura 8.15: Aplicações de todos os filtros discutidos no capítulo 3, com as configurações de parâmetros determinadas na seção 6.9 na imagem de teste Testcase 13-47. No canto inferior direito de cada imagem encontra-se uma região ampliada de uma área de interesse do exame que está sendo analisado. 

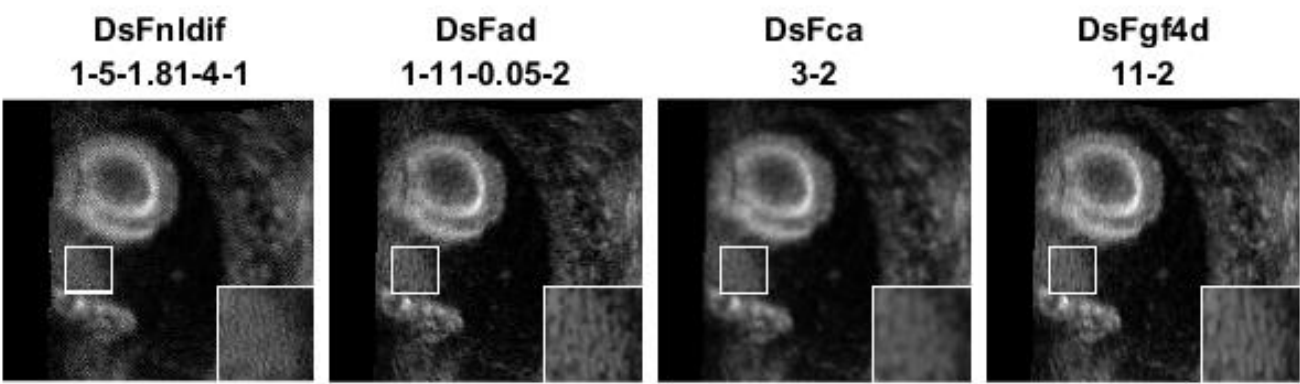

DsFhomog

3-4

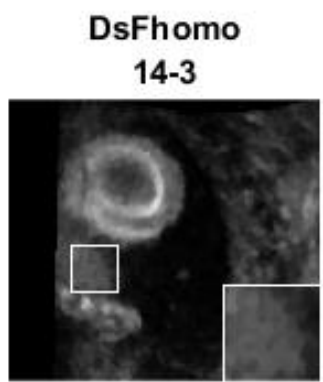

DsFlecasort

2-3

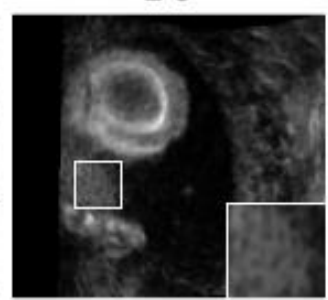

DsFIs

2-3

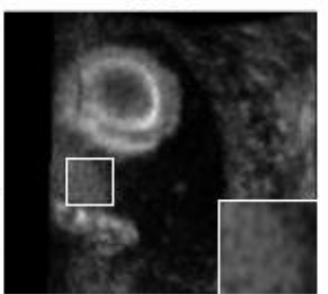

DsFlsminsc

2-5-3
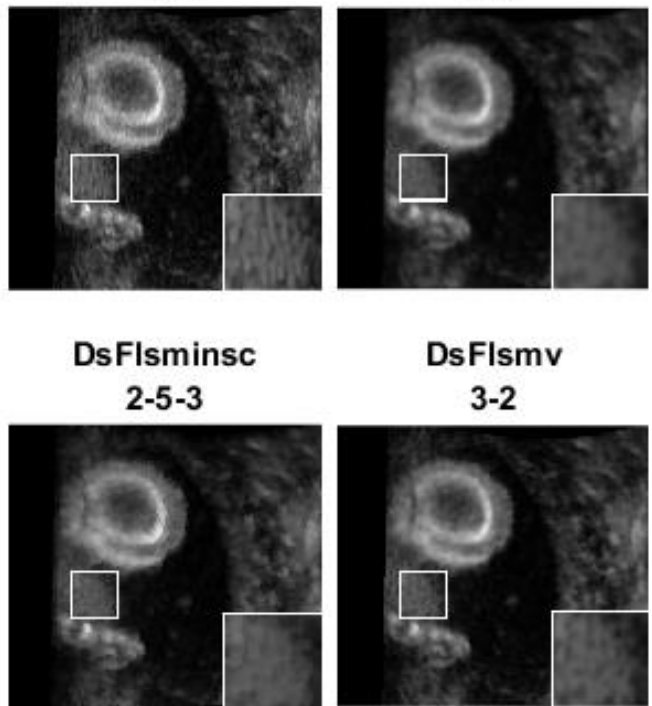

DsFmedian

DsFsrad

Original

Ground Truth

DsFwaveltc

4-7

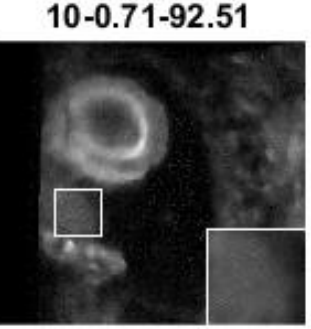

Testcase 14-12

Testcase 14-12

1
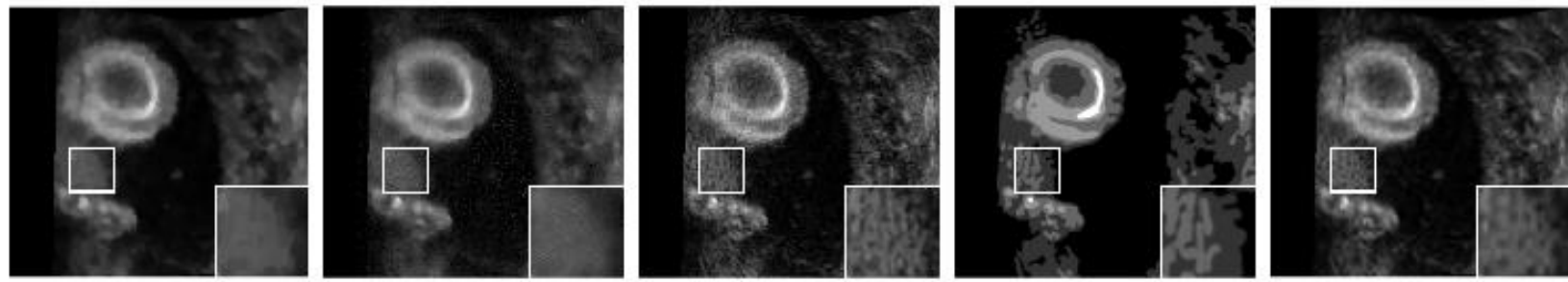

\section{DsFwiener2}

3-3

FoE-Naka

Trilateral

2-1-0.185-0.75
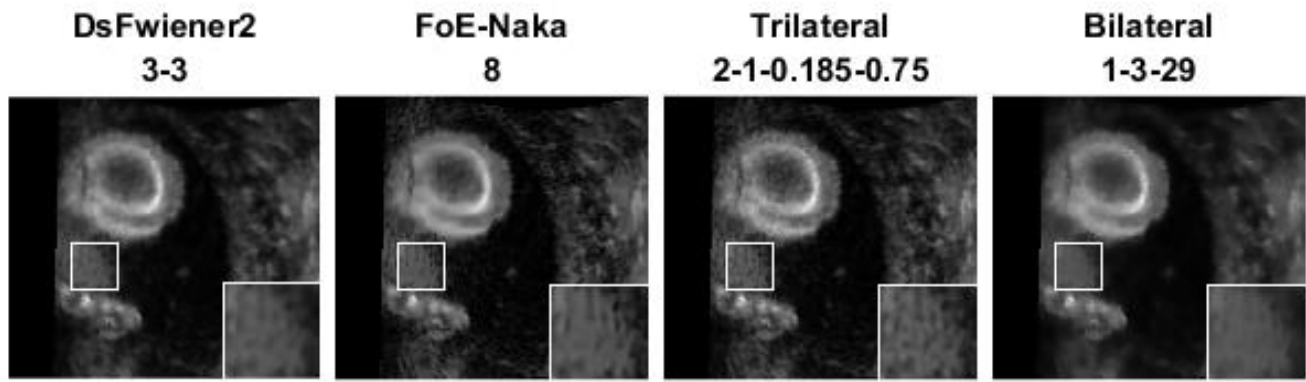

LOSmoothing

1-0.025-8.4

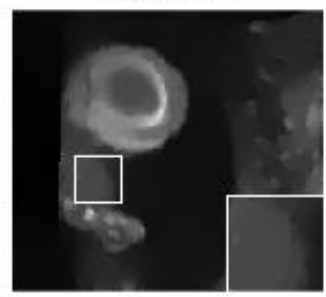

BLF-LO

PPB-Nakagami

1-1-5-0.03-9

1-5-12-5-0.901-0.601
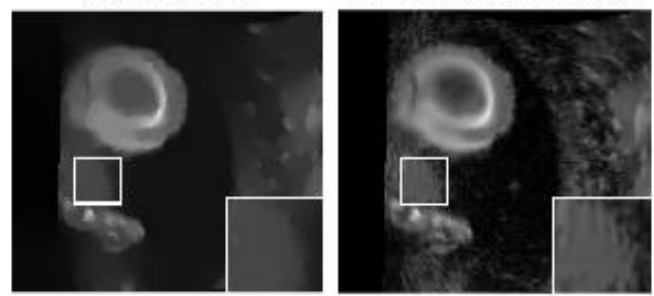

SADCTD

2-0.05

DSDN

1-3-1-2-1
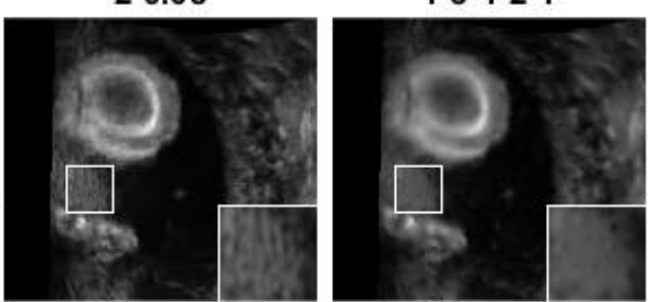

SARBM3D

4-5

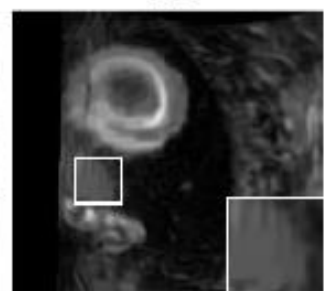

Figura 8.16: Aplicações de todos os filtros discutidos no capítulo 3, com as configurações de parâmetros determinadas na seção 6.9 na imagem de teste Testcase 14-12. No canto inferior direito de cada imagem encontra-se uma região ampliada de uma área de interesse do exame que está sendo analisado. 

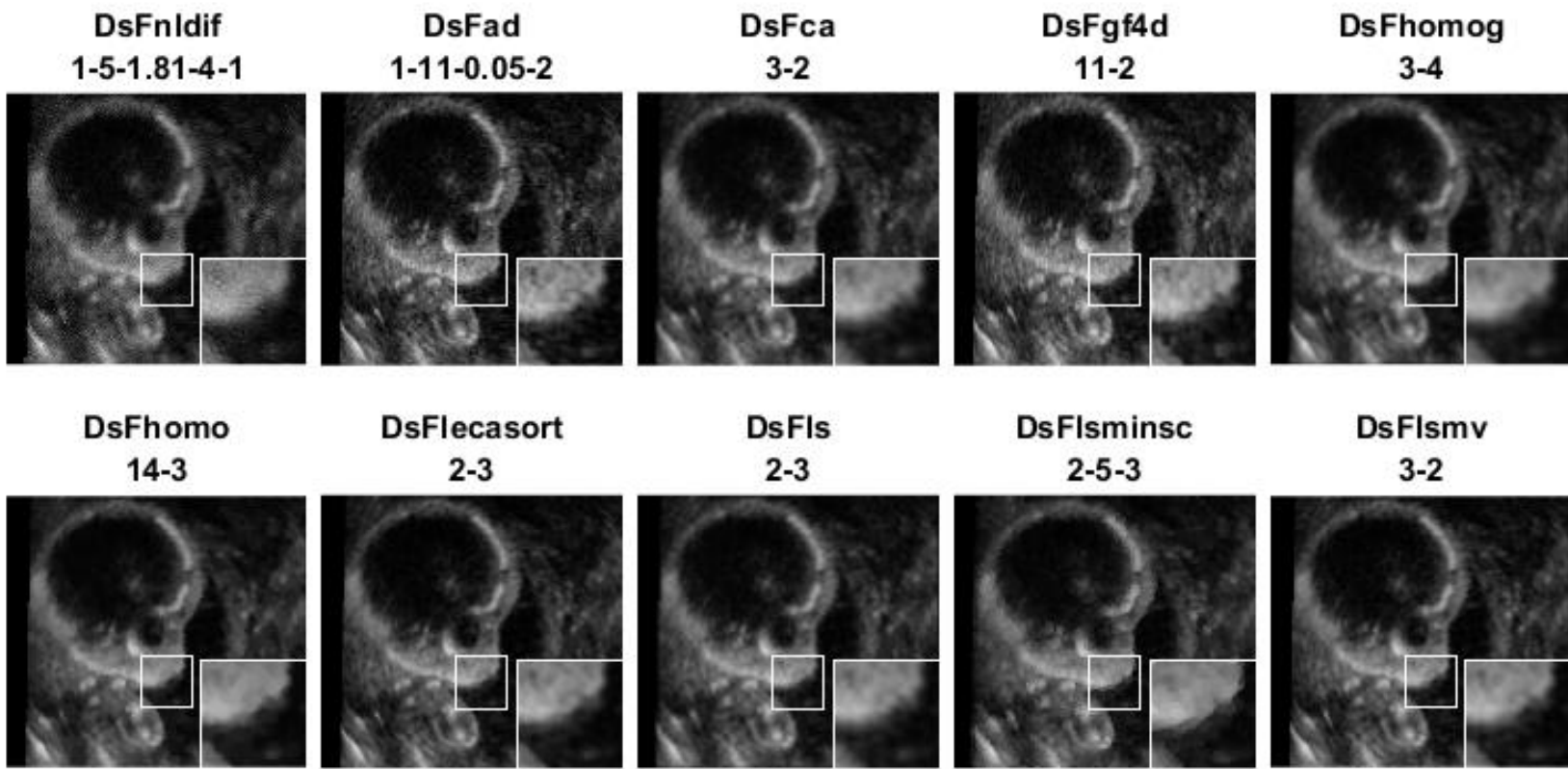

\section{DsFlecasort}

2-3

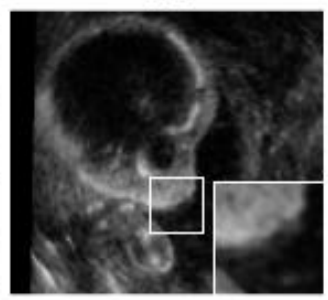

DsFls

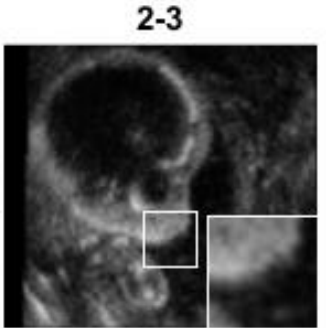

DsFlsminsc

2-5-3

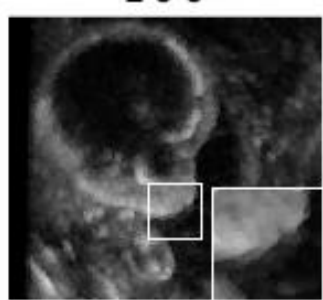

DsFIsmv

DsFmedian

DsFsrad

Original

10-0.71-92.51

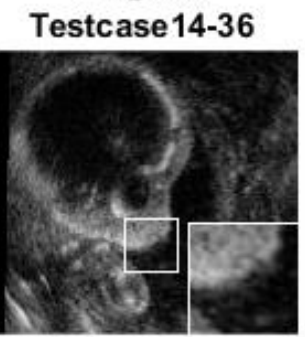

Ground Truth

Testcase 14-36
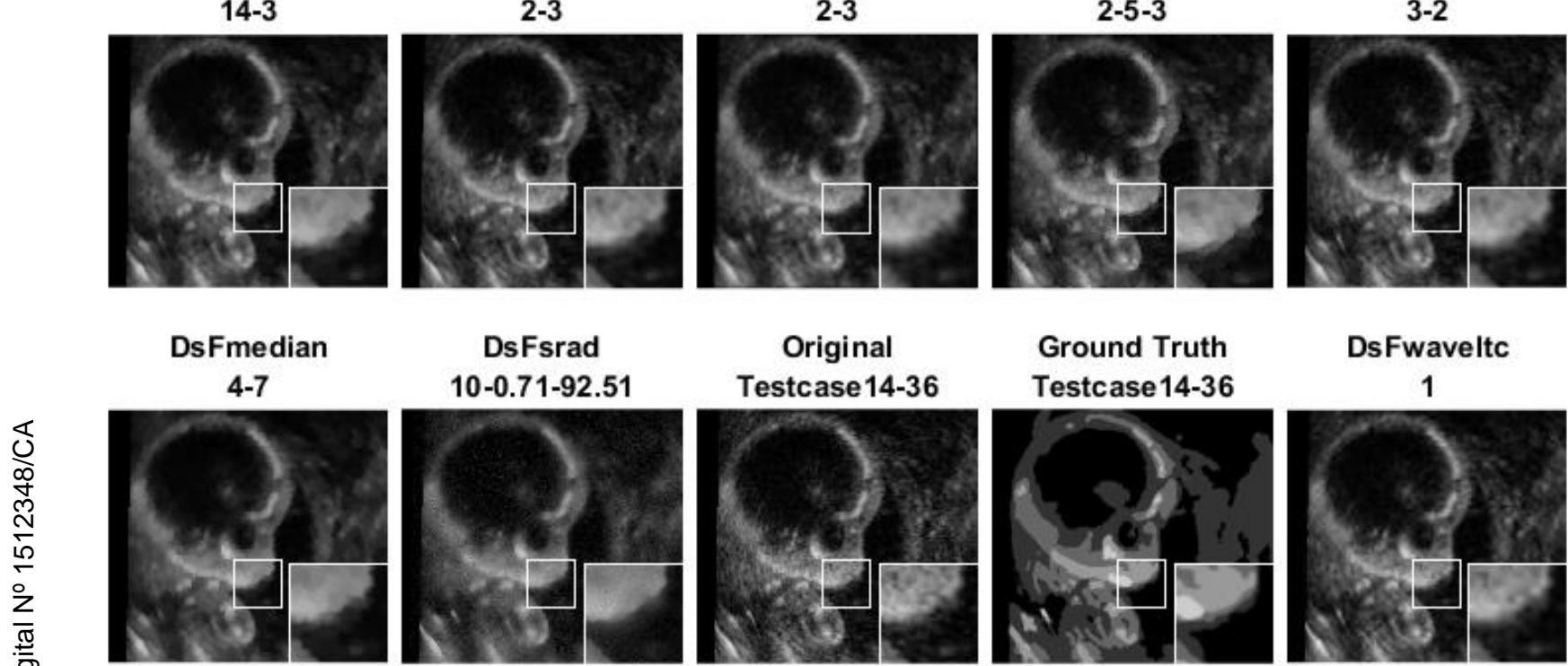

FoE-Naka

8

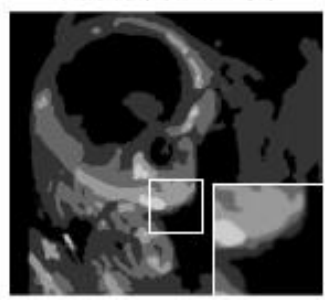

DsFwaveltc

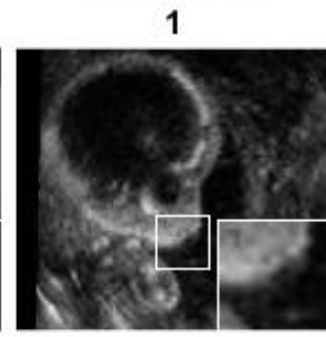

\section{DsFwiener2}

3-3
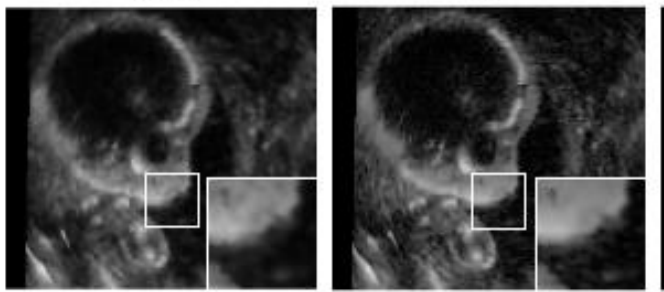

Trilateral

2-1-0.185-0.75

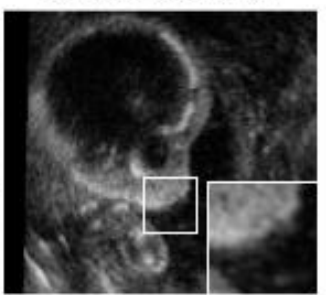

Bilatera

1-3-29

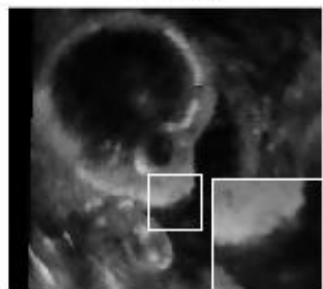

DSDN

PPB-Nakagami

1-1-5-0.03-9
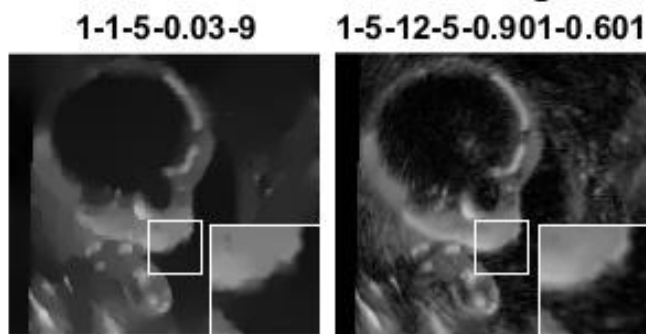

SADCTD

2-0.05

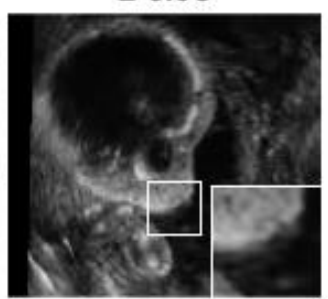

1-3-1-2-1

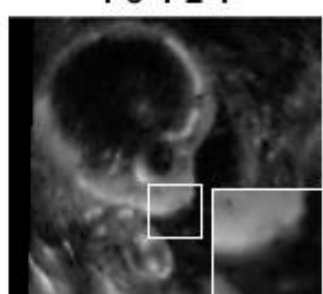

LOSmoothing

1-0.025-8.4

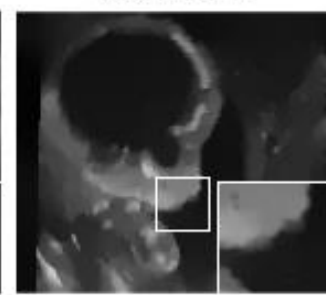

SARBM3D

4-5

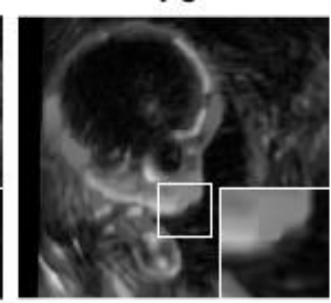

Figura 8.17: Aplicações de todos os filtros discutidos no capítulo 3, com as configurações de parâmetros determinadas na seção 6.9 na imagem de teste Testcase 14-36. No canto inferior direito de cada imagem encontra-se uma região ampliada de uma área de interesse do exame que está sendo analisado. 

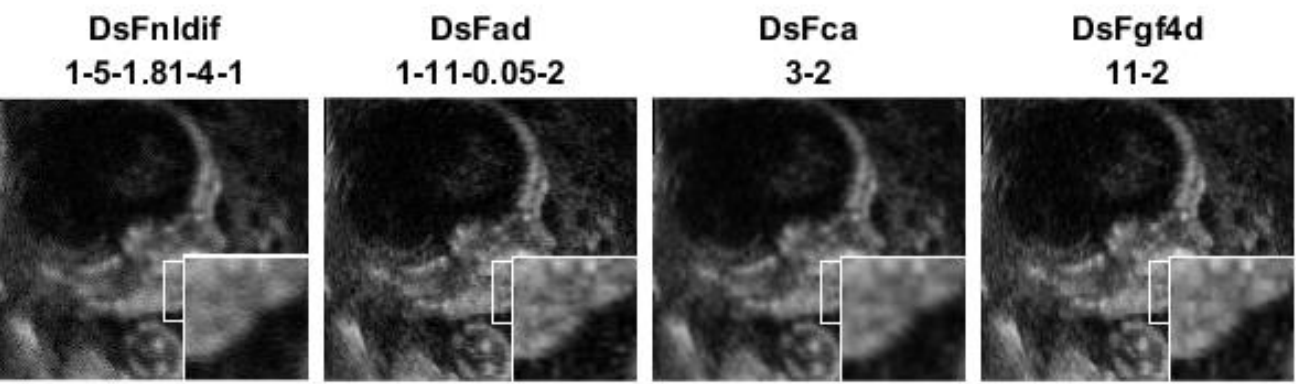

DsFhomog

3-4

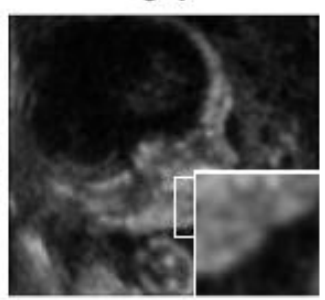

DsFhomo

14-3

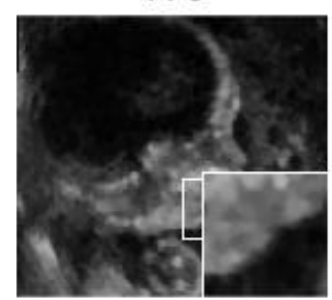

DsFlecasort

2-3

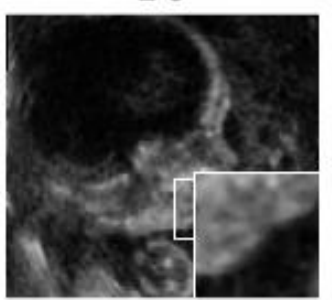

DsFls

2-3

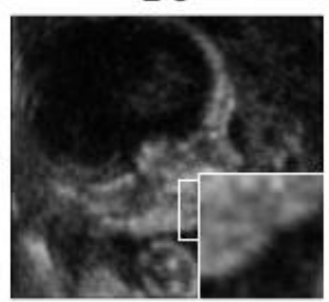

DsFIsminsc

2-5-3
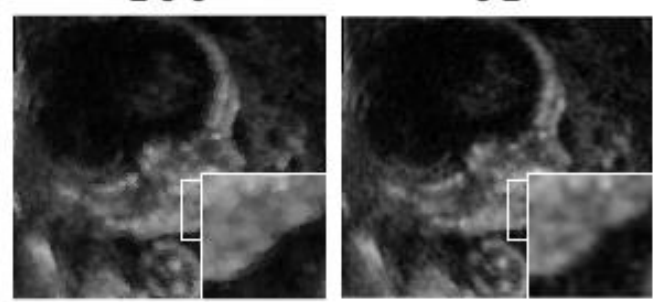

DsFmedian
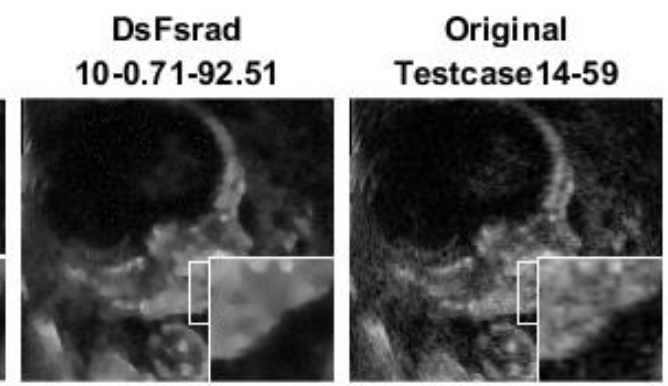

Ground Truth

Testcase14-59

DsFwaveltc

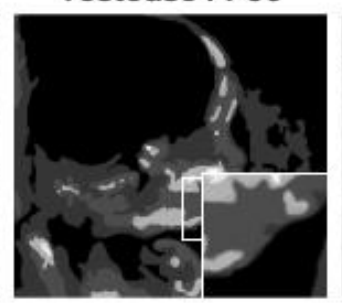

1

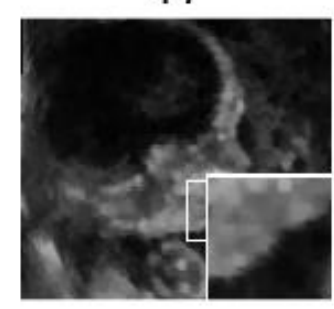

FoE-Naka

8
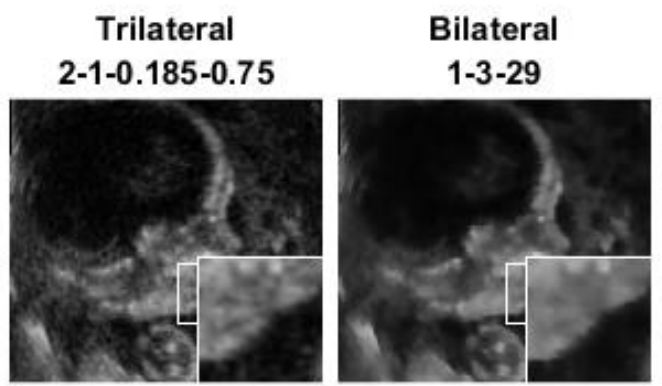

LOSmoothing

1-0.025-8.4
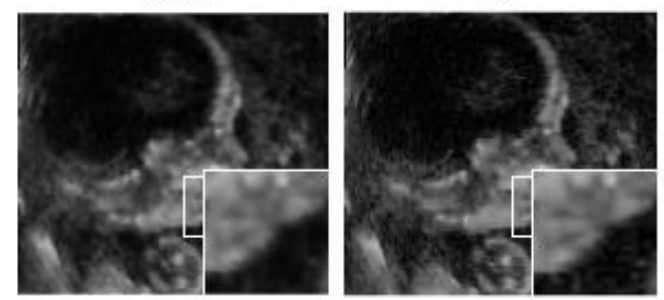

PPB-Nakagami

BLF-LO 1-5-12-5-0.901-0.601

\section{SADCTD}

2-0.05
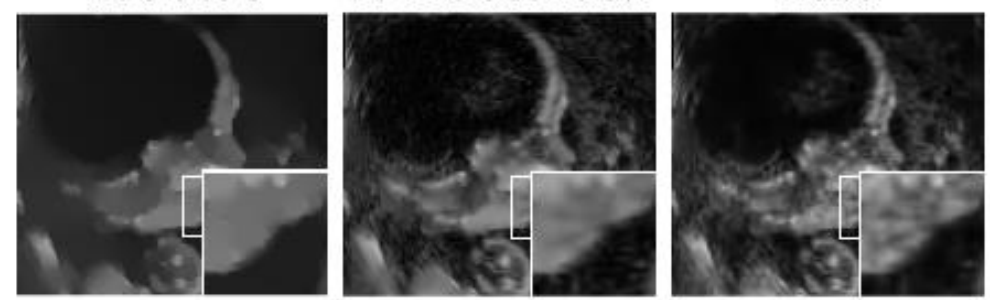

DSDN

$1-3-1-2-1$
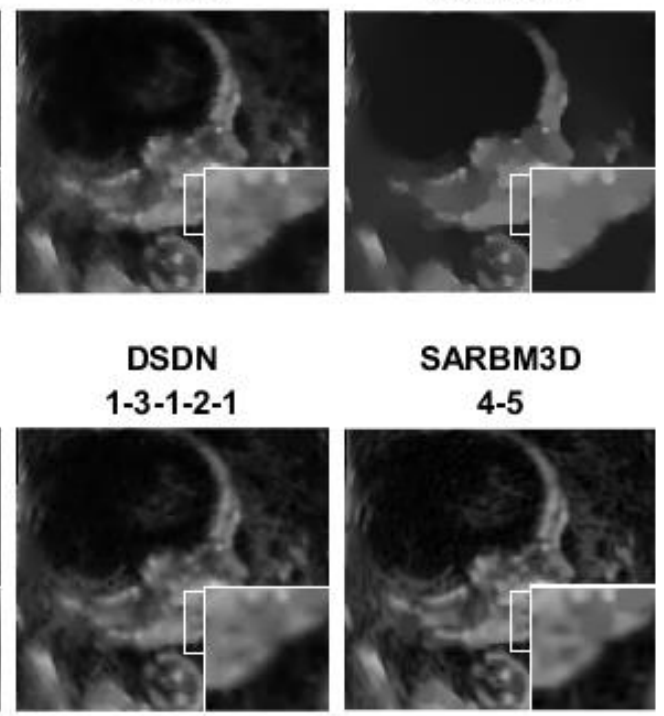

SARBM3D

4-5

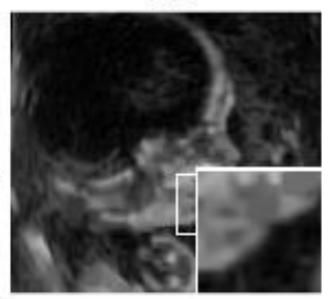

Figura 8.18: Aplicações de todos os filtros discutidos no capítulo 3, com as configurações de parâmetros determinadas na seção 6.9 na imagem de teste Testcase 14-59. No canto inferior direito de cada imagem encontra-se uma região ampliada de uma área de interesse do exame que está sendo analisado. 


\section{Anexo B}

Pixelwise Median of ( Ground Truth - All Filters )
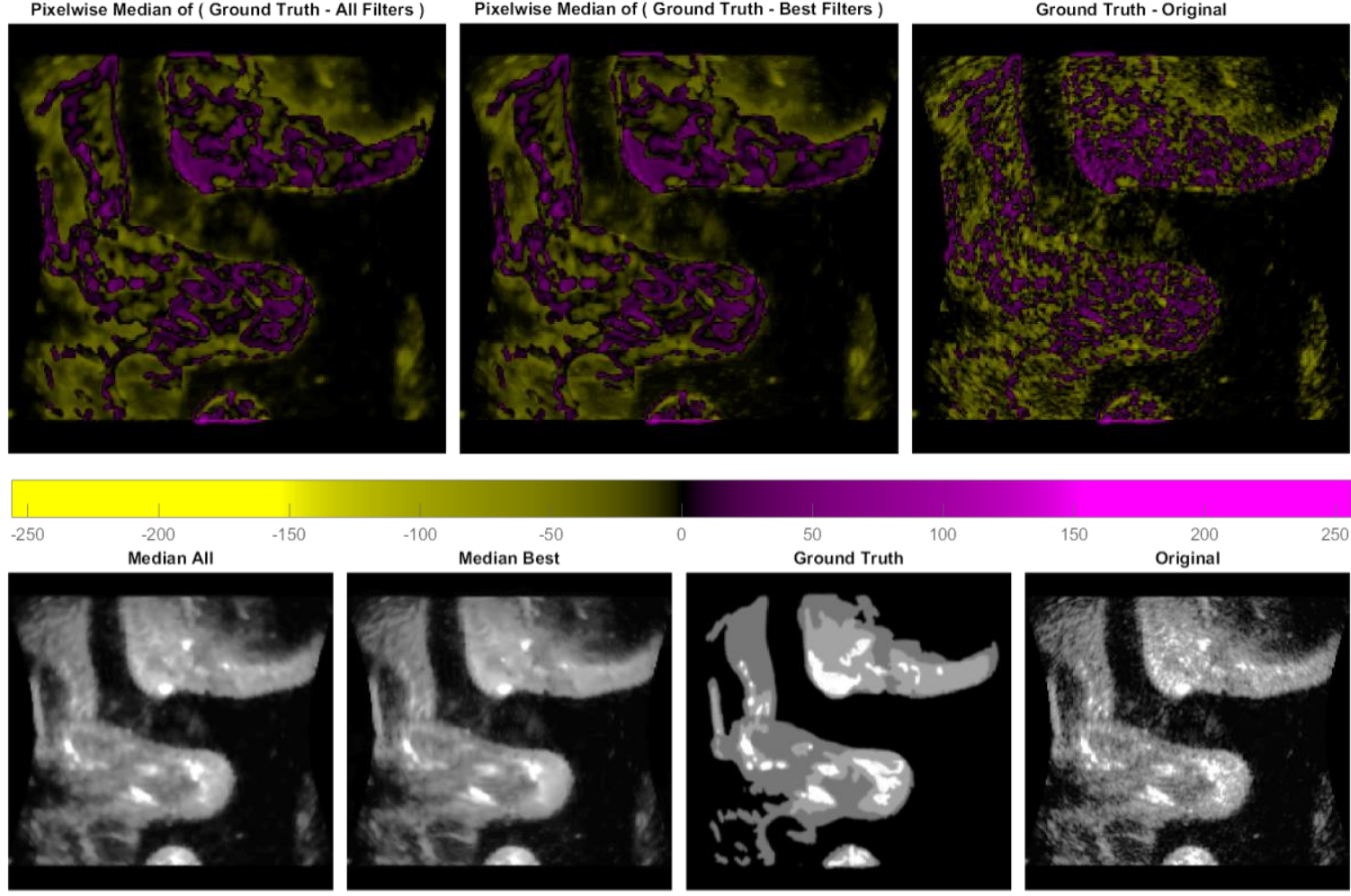
Ground Truth
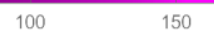

200
Original
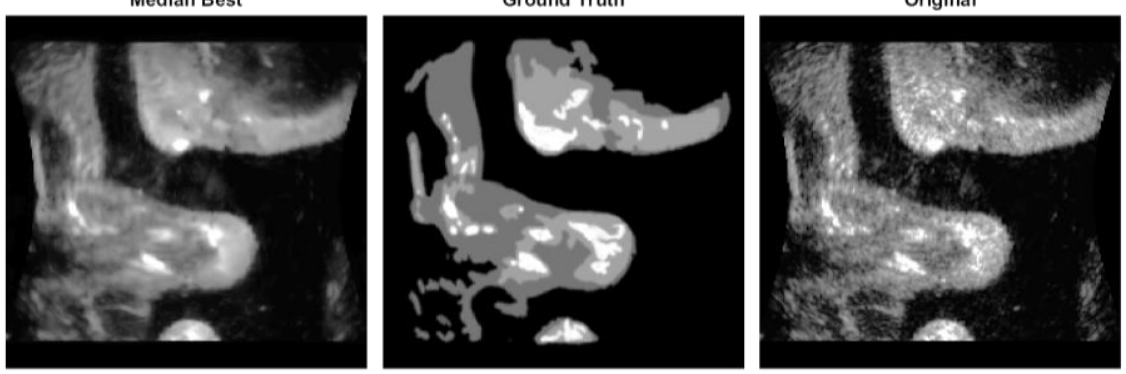

Figura 9.1 - Na linha superior estão as imagens obtidas pela subtração da imagem Ground Truth Testcase01_26 com a mediana, pixel a pixel, do melhor resultado todos os filtros, enquanto na imagem central está a mesma subtração, mas neste caso somente com o melhor resultado dos filtros PPB_Nakagami, SADCTD, SARBM3D, BLF_L0, DSDN, L0 e FoE_Naka e na imagem à direita está a subtração da imagem ground truth com a imagem original. Na linha inferior encontram-se as imagens medianas respectivas, assim como as imagens de Grouth Truth e Original. 

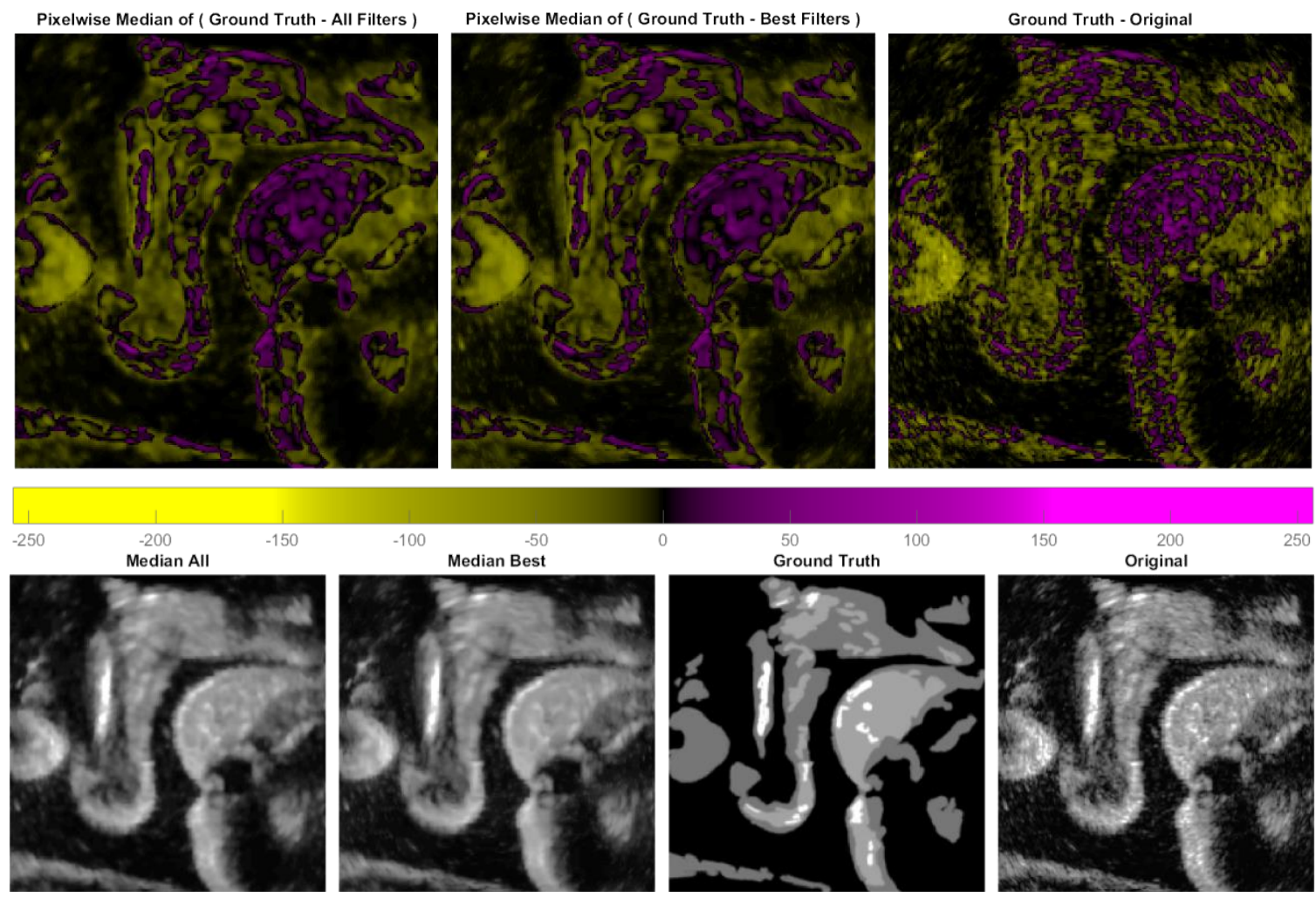

Figura 9.2 - Na linha superior estão as imagens obtidas pela subtração da imagem Ground Truth Testcase01_46 com a mediana, pixel a pixel, do melhor resultado todos os filtros, enquanto na imagem central está a mesma subtração, mas neste caso somente com o melhor resultado dos filtros PPB_Nakagami, SADCTD, SARBM3D, BLF_L0, DSDN, LO e FoE_Naka e na imagem à direita está a subtração da imagem ground truth com a imagem original. Na linha inferior encontram-se as imagens medianas respectivas, assim como as imagens de Grouth Truth e Original. 

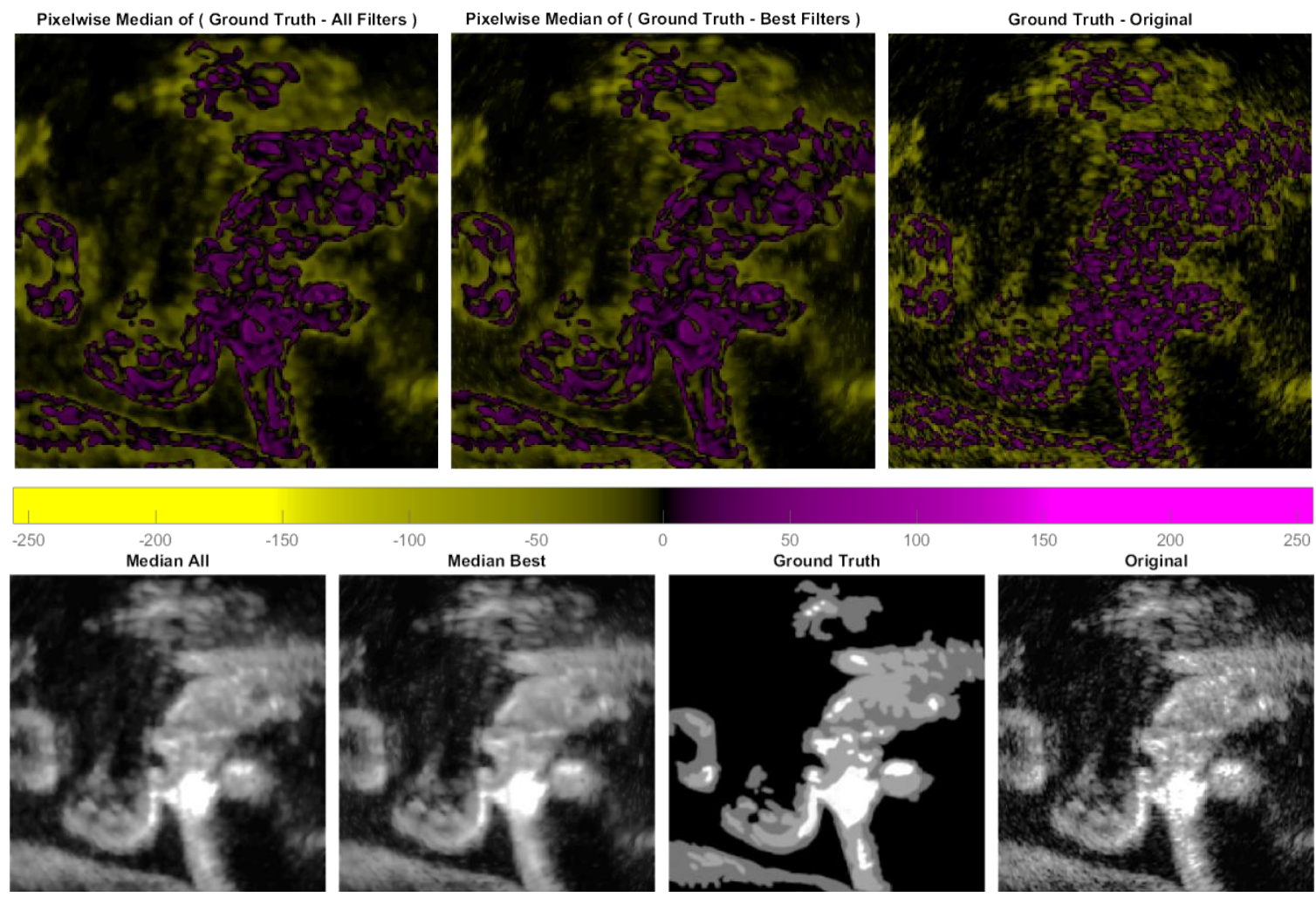

Figura 9.3 - Na linha superior estão as imagens obtidas pela subtração da imagem Ground Truth Testcase01_62 com a mediana, pixel a pixel, do melhor resultado todos os filtros, enquanto na imagem central está a mesma subtração, mas neste caso somente com o melhor resultado dos filtros PPB_Nakagami, SADCTD, SARBM3D, BLF_LO, DSDN, LO e FoE_Naka e na imagem à direita está a subtração da imagem ground truth com a imagem original. Na linha inferior encontram-se as imagens medianas respectivas, assim como as imagens de Grouth Truth e Original. 

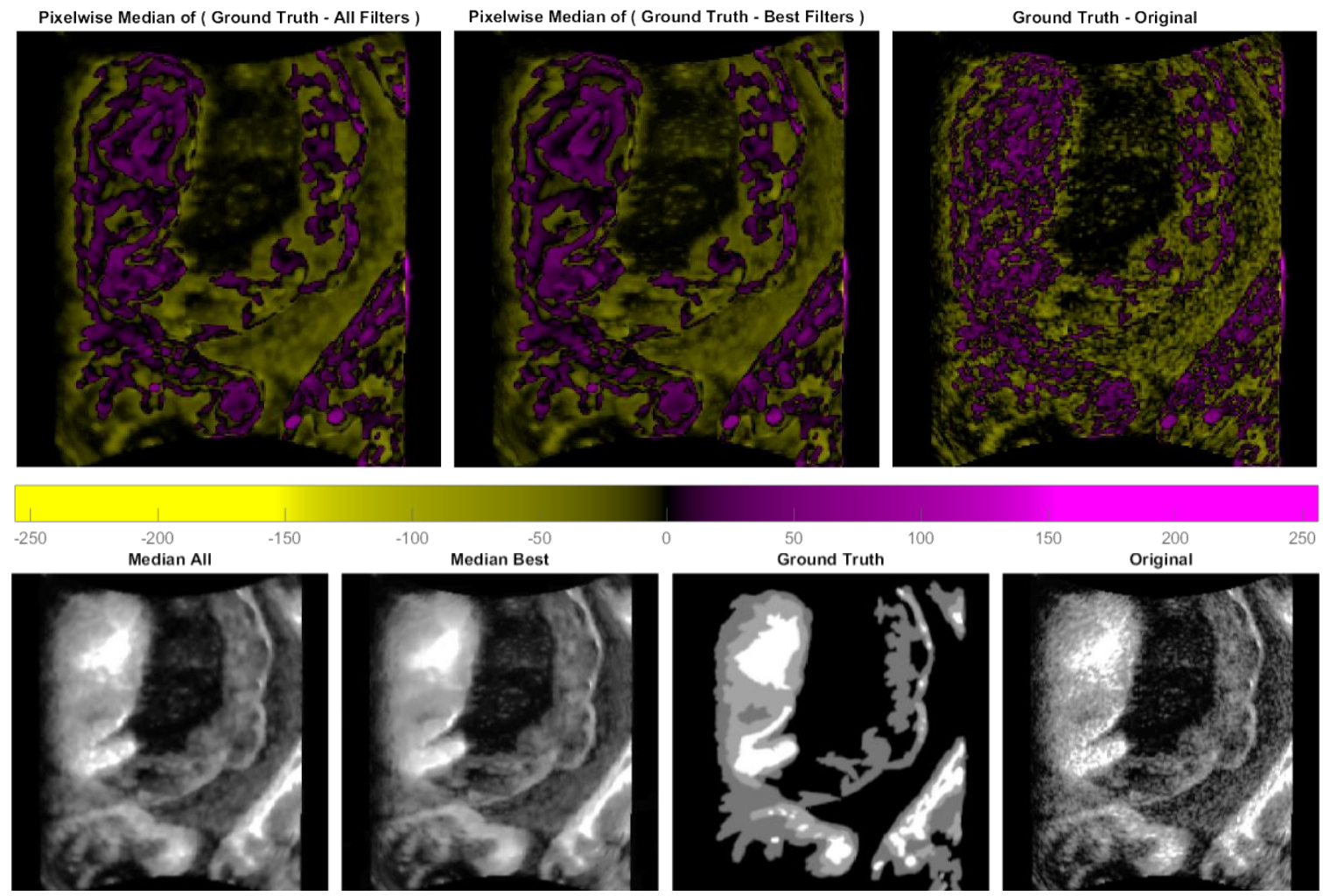

Figura 9.4 - Na linha superior estão as imagens obtidas pela subtração da imagem Ground Truth Testcase02_12 com a mediana, pixel a pixel, do melhor resultado todos os filtros, enquanto na imagem central está a mesma subtração, mas neste caso somente com o melhor resultado dos filtros PPB_Nakagami, SADCTD, SARBM3D, BLF_L0, DSDN, L0 e FoE_Naka e na imagem à direita está a subtração da imagem ground truth com a imagem original. Na linha inferior encontram-se as imagens medianas respectivas, assim como as imagens de Grouth Truth e Original. 

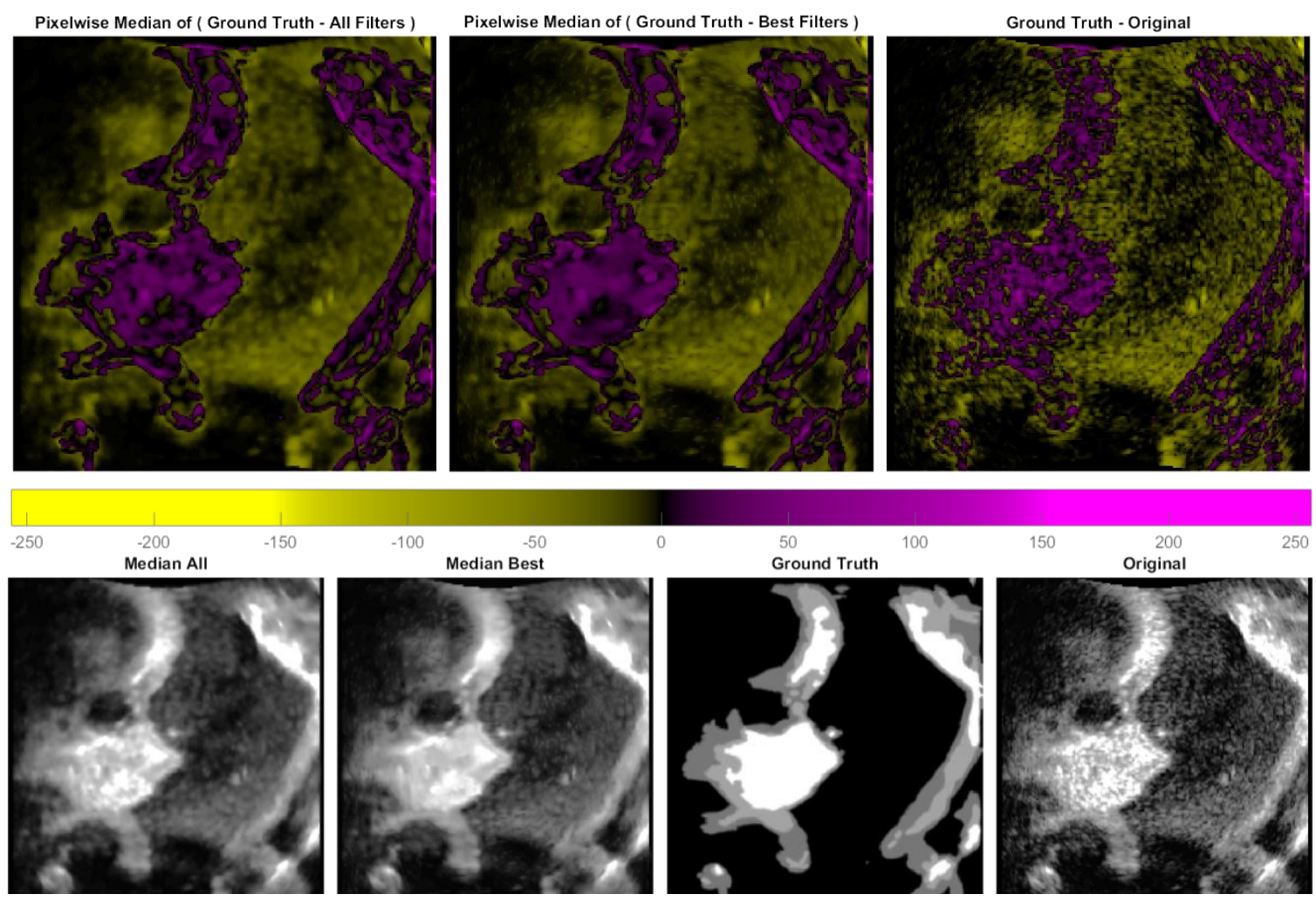

Figura 9.5 - Na linha superior estão as imagens obtidas pela subtração da imagem Ground Truth Testcase02_29 com a mediana, pixel a pixel, do melhor resultado todos os filtros, enquanto na imagem central está a mesma subtração, mas neste caso somente com o melhor resultado dos filtros PPB_Nakagami, SADCTD, SARBM3D, BLF_L0, DSDN, LO e FoE_Naka e na imagem à direita está a subtração da imagem ground truth com a imagem original. Na linha inferior encontram-se as imagens medianas respectivas, assim como as imagens de Grouth Truth e Original. 

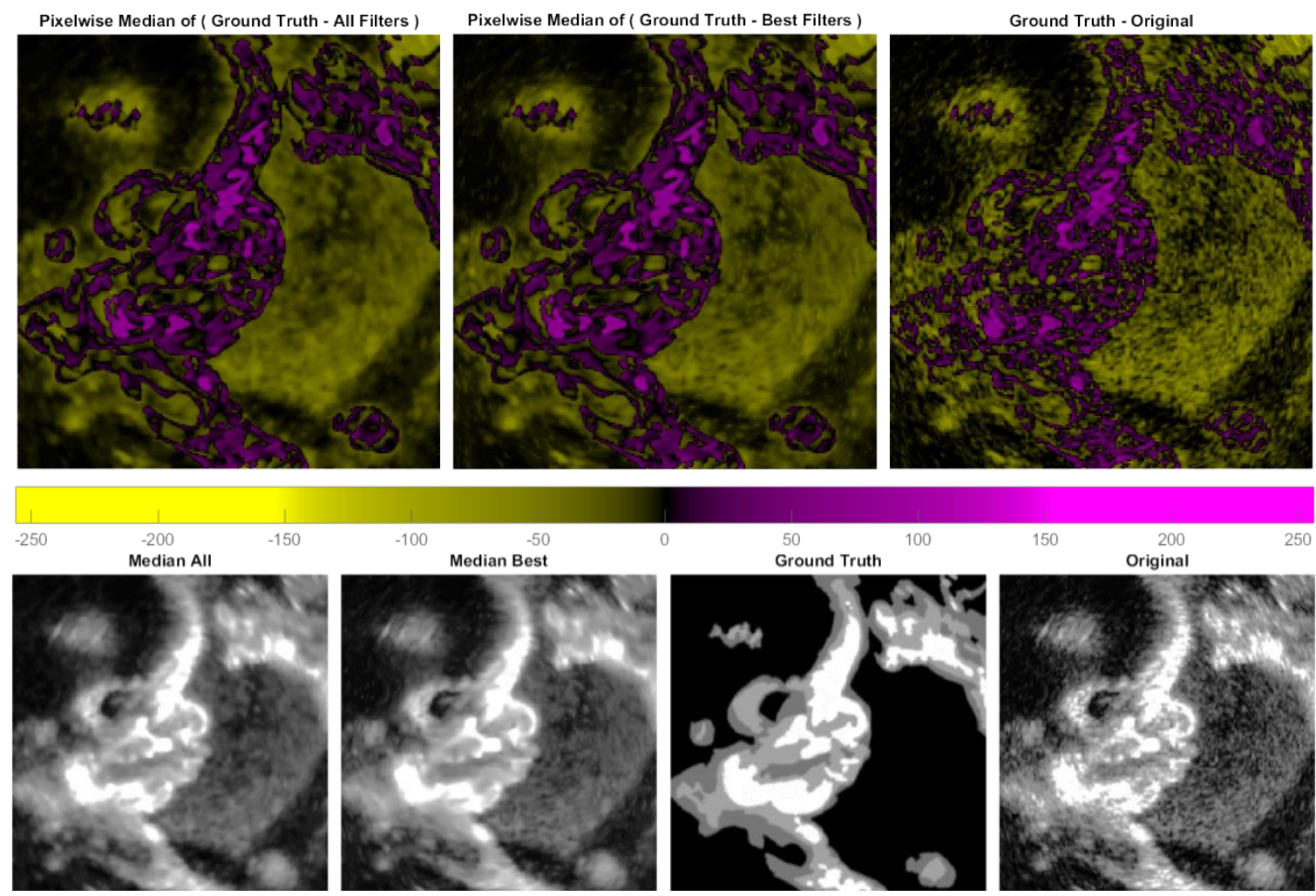

Figura 9.6 - Na linha superior estão as imagens obtidas pela subtração da imagem Ground Truth Testcase02_45 com a mediana, pixel a pixel, do melhor resultado todos os filtros, enquanto na imagem central está a mesma subtração, mas neste caso somente com o melhor resultado dos filtros PPB_Nakagami, SADCTD, SARBM3D, BLF_L0, DSDN, LO e FoE_Naka e na imagem à direita está a subtração da imagem ground truth com a imagem original. Na linha inferior encontram-se as imagens medianas respectivas, assim como as imagens de Grouth Truth e Original. 
Pixelwise Median of ( Ground Truth - All Filters )
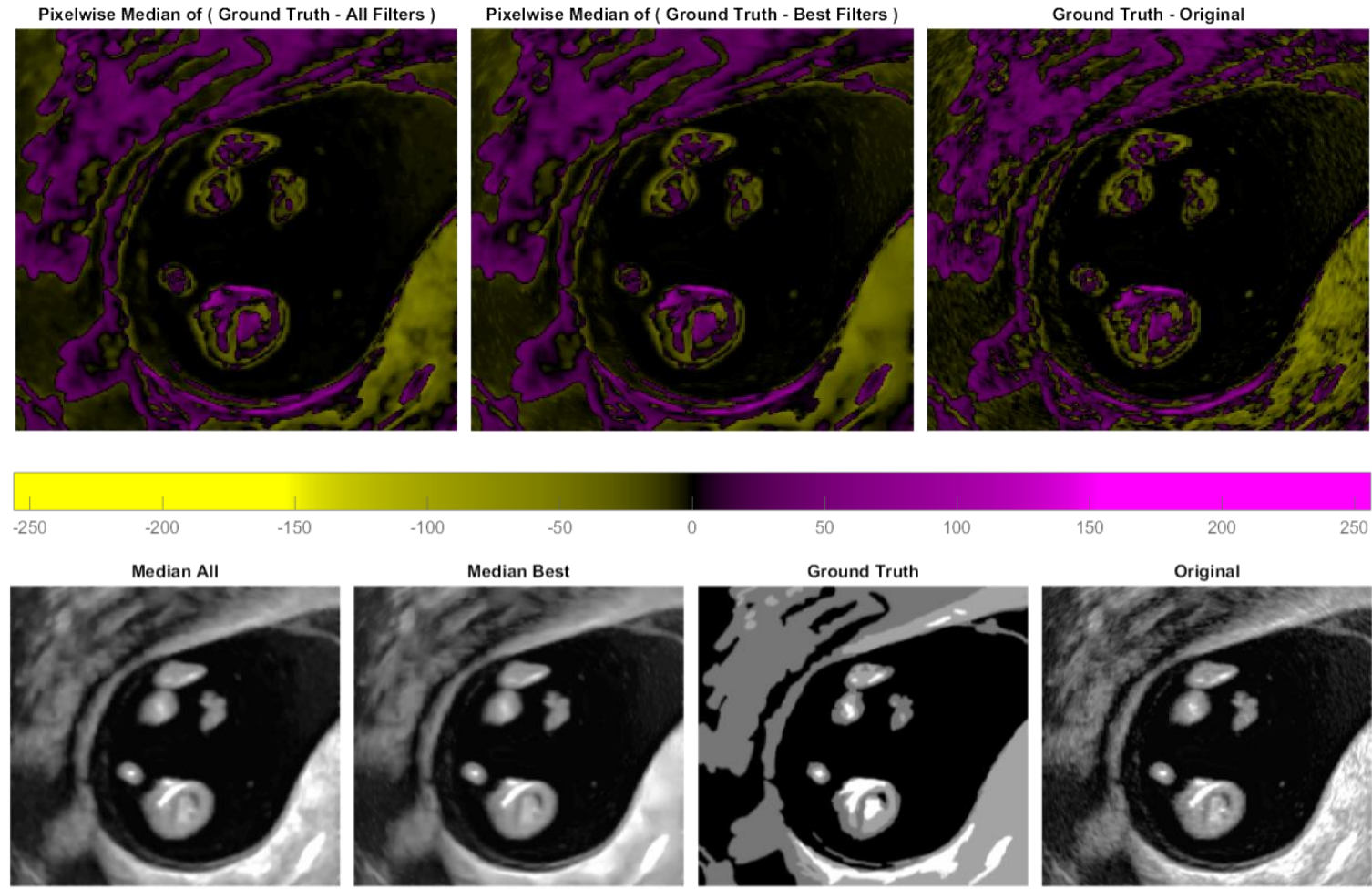

Median Best
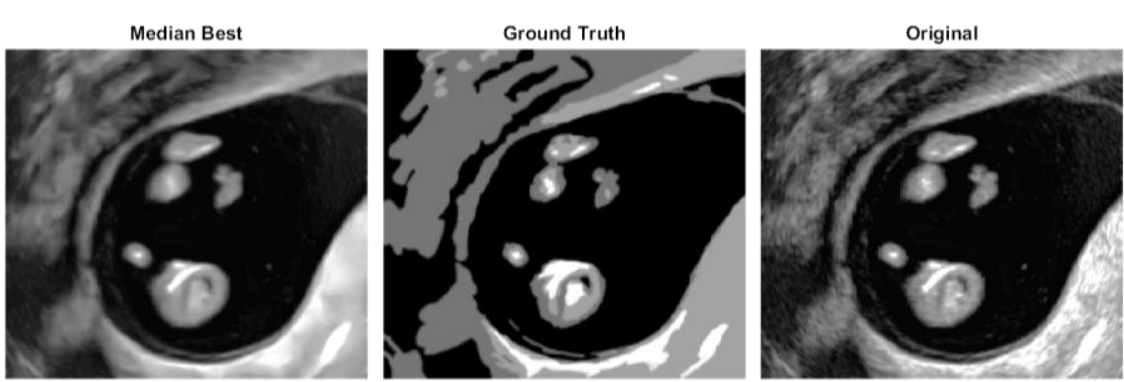

Figura 9.7 - Na linha superior estão as imagens obtidas pela subtração da imagem Ground Truth Testcase03_29 com a mediana, pixel a pixel, do melhor resultado todos os filtros, enquanto na imagem central está a mesma subtração, mas neste caso somente com o melhor resultado dos filtros PPB_Nakagami, SADCTD, SARBM3D, BLF_L0, DSDN, LO e FoE_Naka e na imagem à direita está a subtração da imagem ground truth com a imagem original. Na linha inferior encontram-se as imagens medianas respectivas, assim como as imagens de Grouth Truth e Original. 

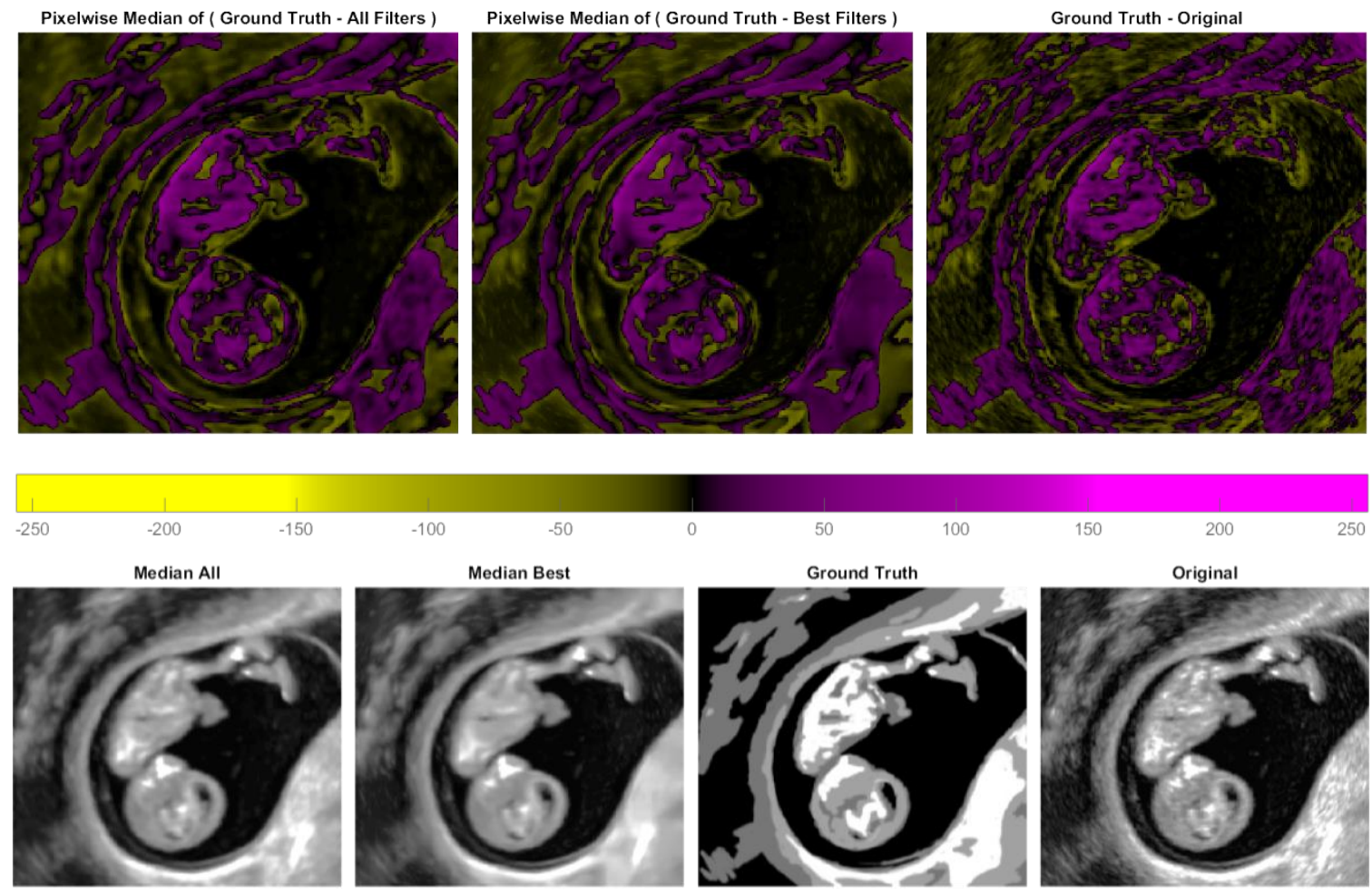

Figura 9.8 - Na linha superior estão as imagens obtidas pela subtração da imagem Ground Truth Testcase03_44 com a mediana, pixel a pixel, do melhor resultado todos os filtros, enquanto na imagem central está a mesma subtração, mas neste caso somente com o melhor resultado dos filtros PPB_Nakagami, SADCTD, SARBM3D, BLF_L0, DSDN, LO e FoE_Naka e na imagem à direita está a subtração da imagem ground truth com a imagem original. Na linha inferior encontram-se as imagens medianas respectivas, assim como as imagens de Grouth Truth e Original. 
Pixelwise Median of ( Ground Truth - All Filters )

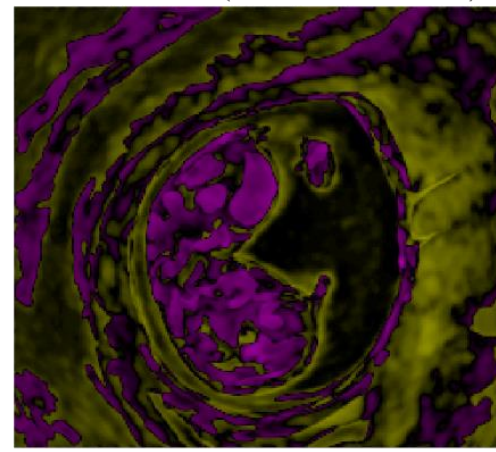

Pixelwise Median of ( Ground Truth - Best Filters )

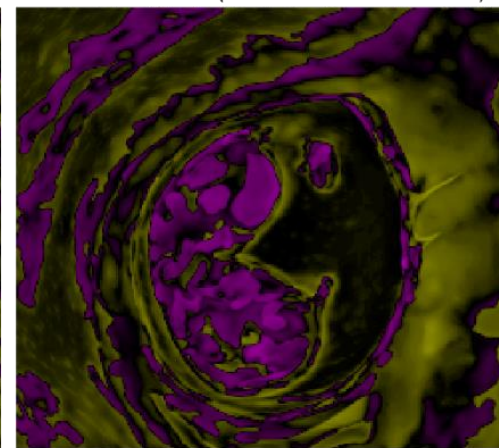

Ground Truth - Original

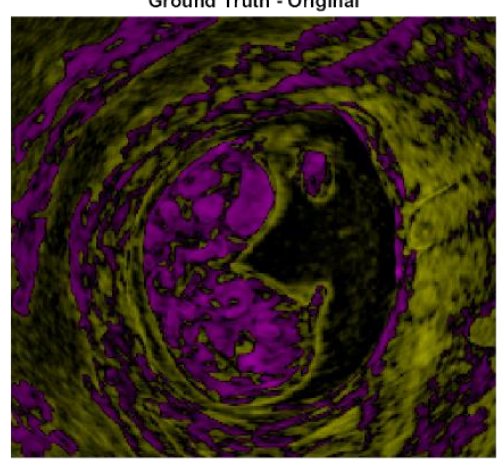

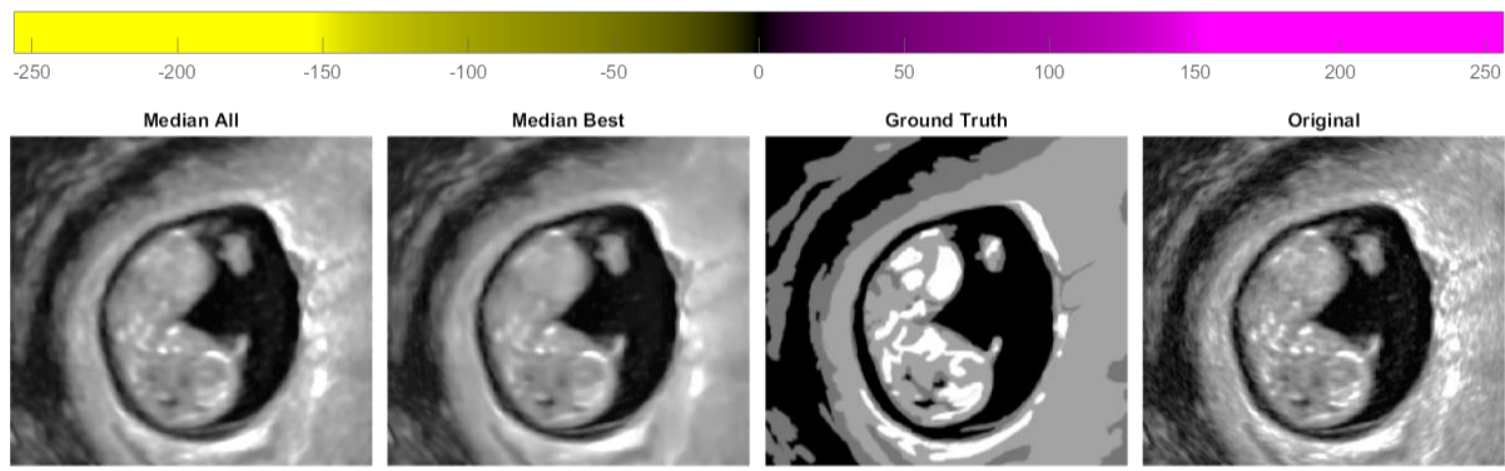

Figura 9.9 - Na linha superior estão as imagens obtidas pela subtração da imagem Ground Truth Testcase03_61 com a mediana, pixel a pixel, do melhor resultado todos os filtros, enquanto na imagem central está a mesma subtração, mas neste caso somente com o melhor resultado dos filtros PPB_Nakagami, SADCTD, SARBM3D, BLF_L0, DSDN, LO e FoE_Naka e na imagem à direita está a subtração da imagem ground truth com a imagem original. Na linha inferior encontram-se as imagens medianas respectivas, assim como as imagens de Grouth Truth e Original. 

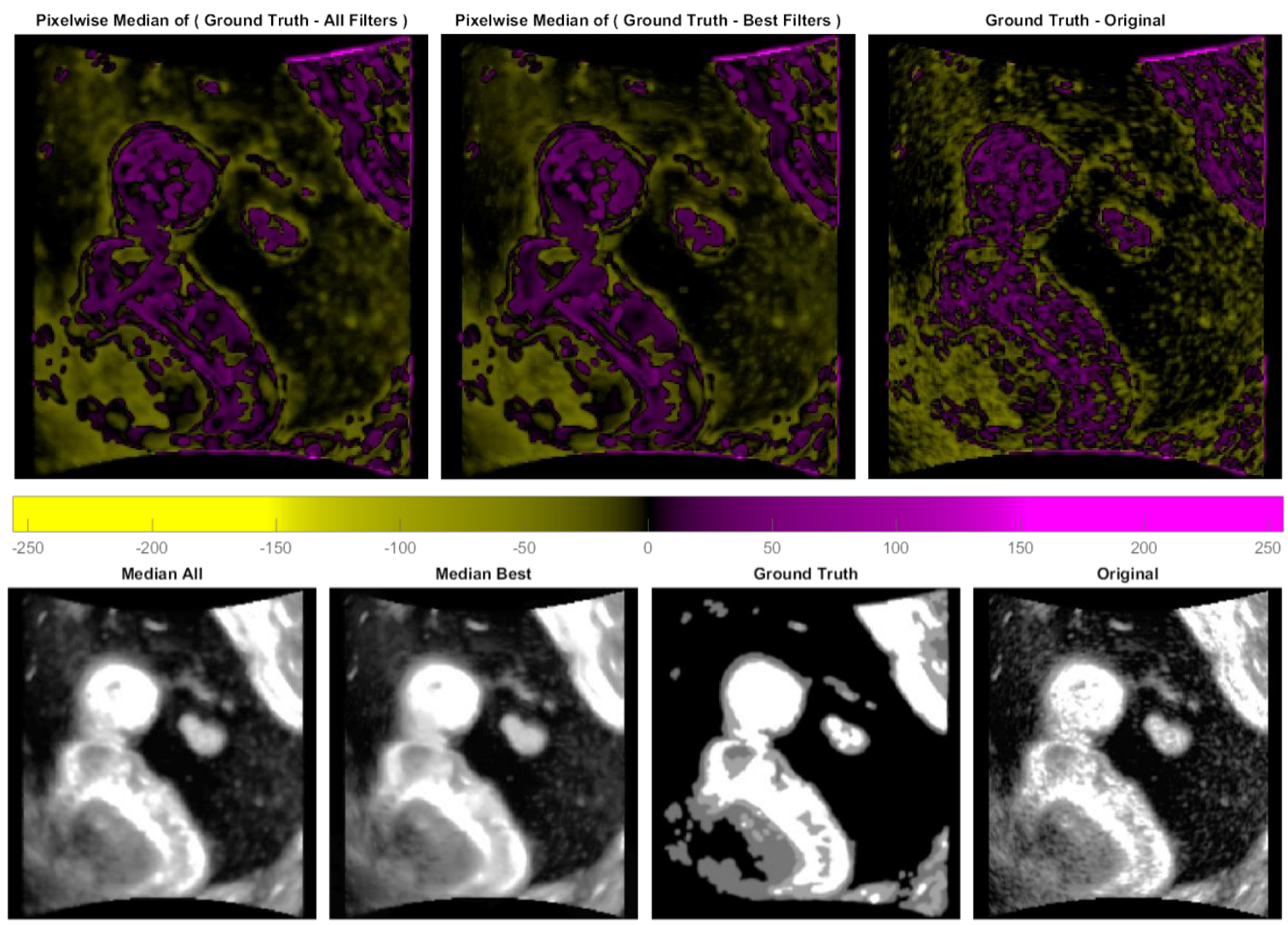

Figura 9.10 - Na linha superior estão as imagens obtidas pela subtração da imagem Ground Truth Testcase04_38 com a mediana, pixel a pixel, do melhor resultado todos os filtros, enquanto na imagem central está a mesma subtração, mas neste caso somente com o melhor resultado dos filtros PPB_Nakagami, SADCTD, SARBM3D, BLF_L0, DSDN, L0 e FoE_Naka e na imagem à direita está a subtração da imagem ground truth com a imagem original. Na linha inferior encontram-se as imagens medianas respectivas, assim como as imagens de Grouth Truth e Original. 

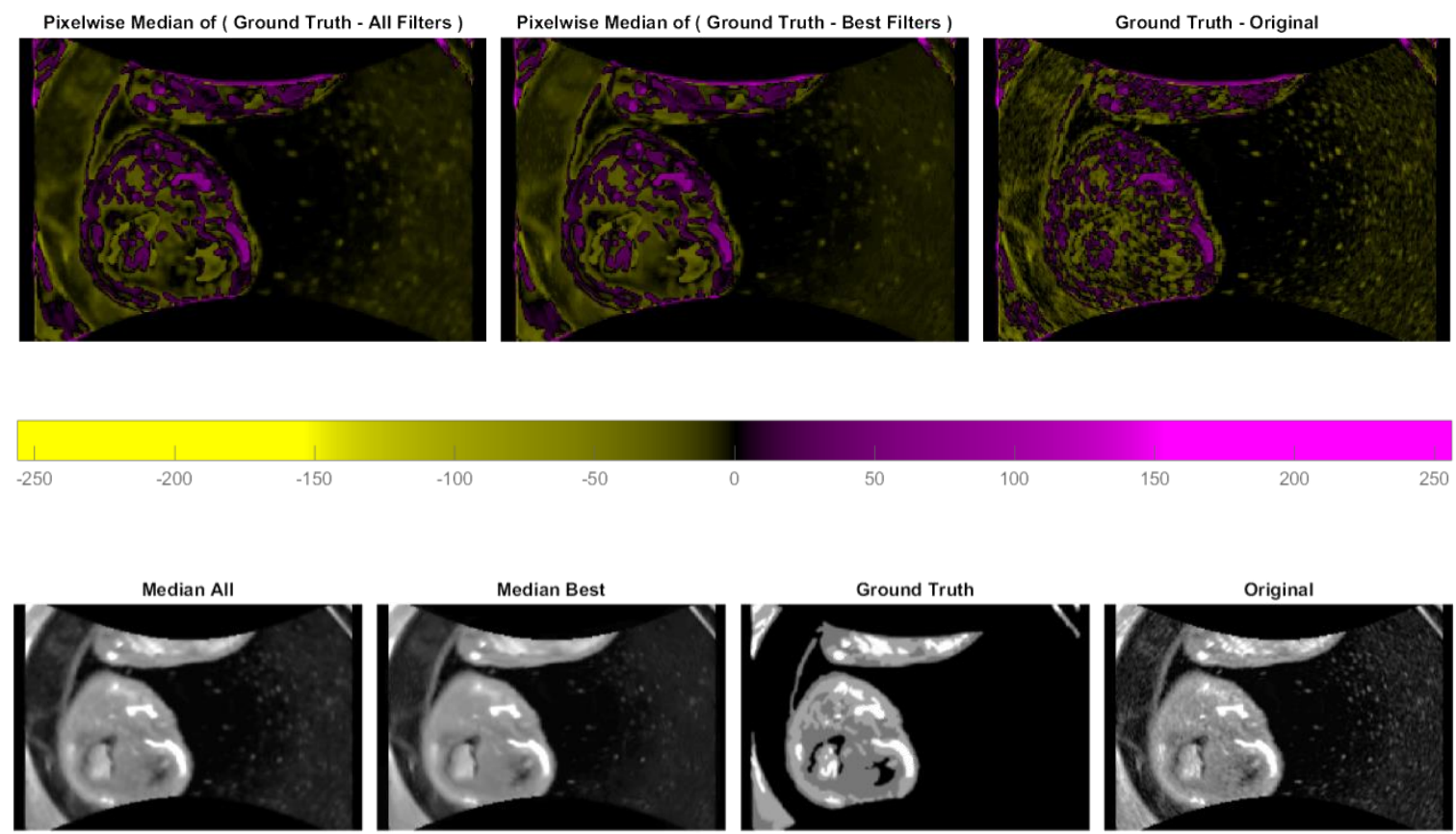

Figura 9.11 - Na linha superior estão as imagens obtidas pela subtração da imagem Ground Truth Testcase05_26 com a mediana, pixel a pixel, do melhor resultado todos os filtros, enquanto na imagem central está a mesma subtração, mas neste caso somente com o melhor resultado dos filtros PPB_Nakagami, SADCTD, SARBM3D, BLF_L0, DSDN, LO e FoE_Naka e na imagem à direita está a subtração da imagem ground truth com a imagem original. Na linha inferior encontram-se as imagens medianas respectivas, assim como as imagens de Grouth Truth e Original. 

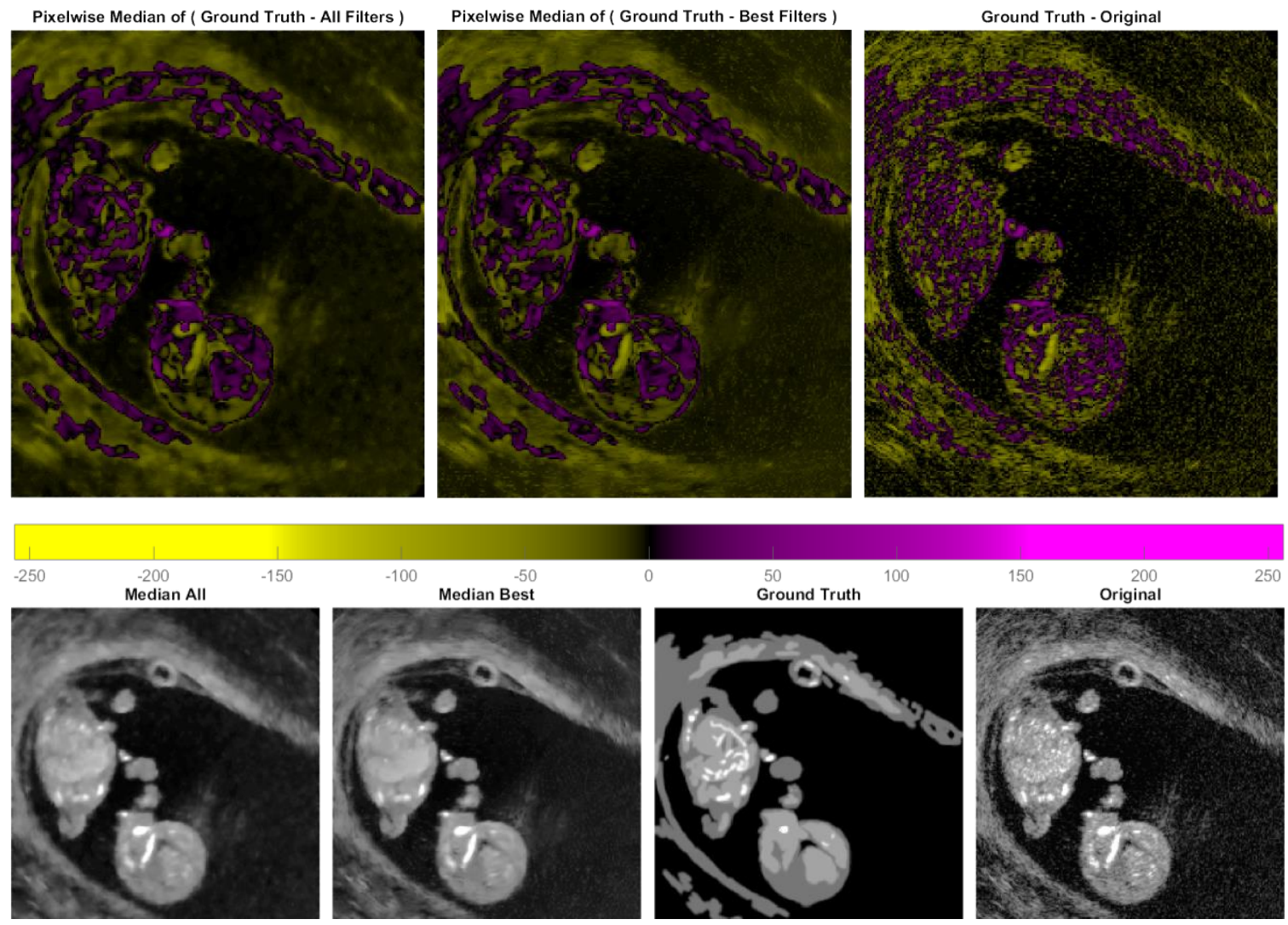

Figura 9.12 - Na linha superior estão as imagens obtidas pela subtração da imagem Ground Truth Testcase07_35 com a mediana, pixel a pixel, do melhor resultado todos os filtros, enquanto na imagem central está a mesma subtração, mas neste caso somente com o melhor resultado dos filtros PPB_Nakagami, SADCTD, SARBM3D, BLF_LO, DSDN, L0 e FoE_Naka e na imagem à direita está a subtração da imagem ground truth com a imagem original. Na linha inferior encontram-se as imagens medianas respectivas, assim como as imagens de Grouth Truth e Original. 
Pixelwise Median of ( Ground Truth - All Filters )

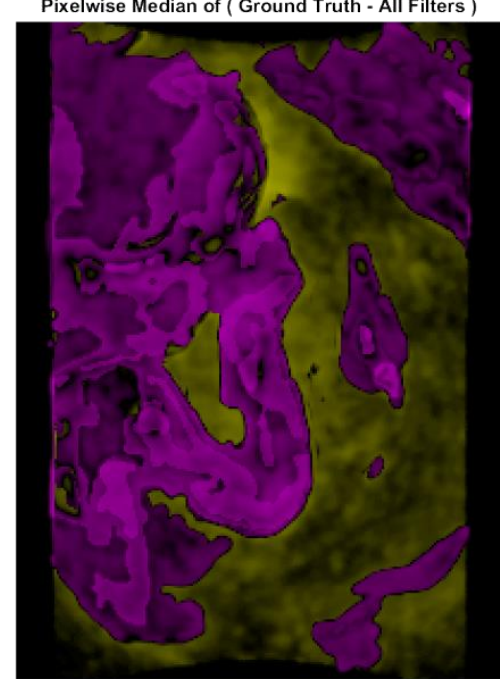

Pixelwise Median of ( Ground Truth - Best Filters )

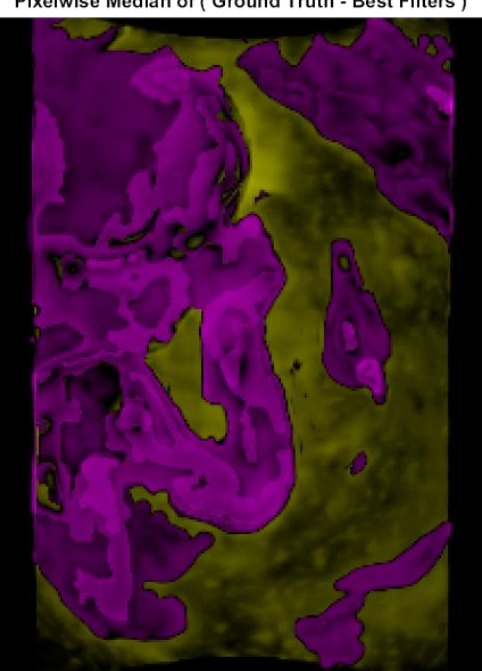

Ground Truth - Original
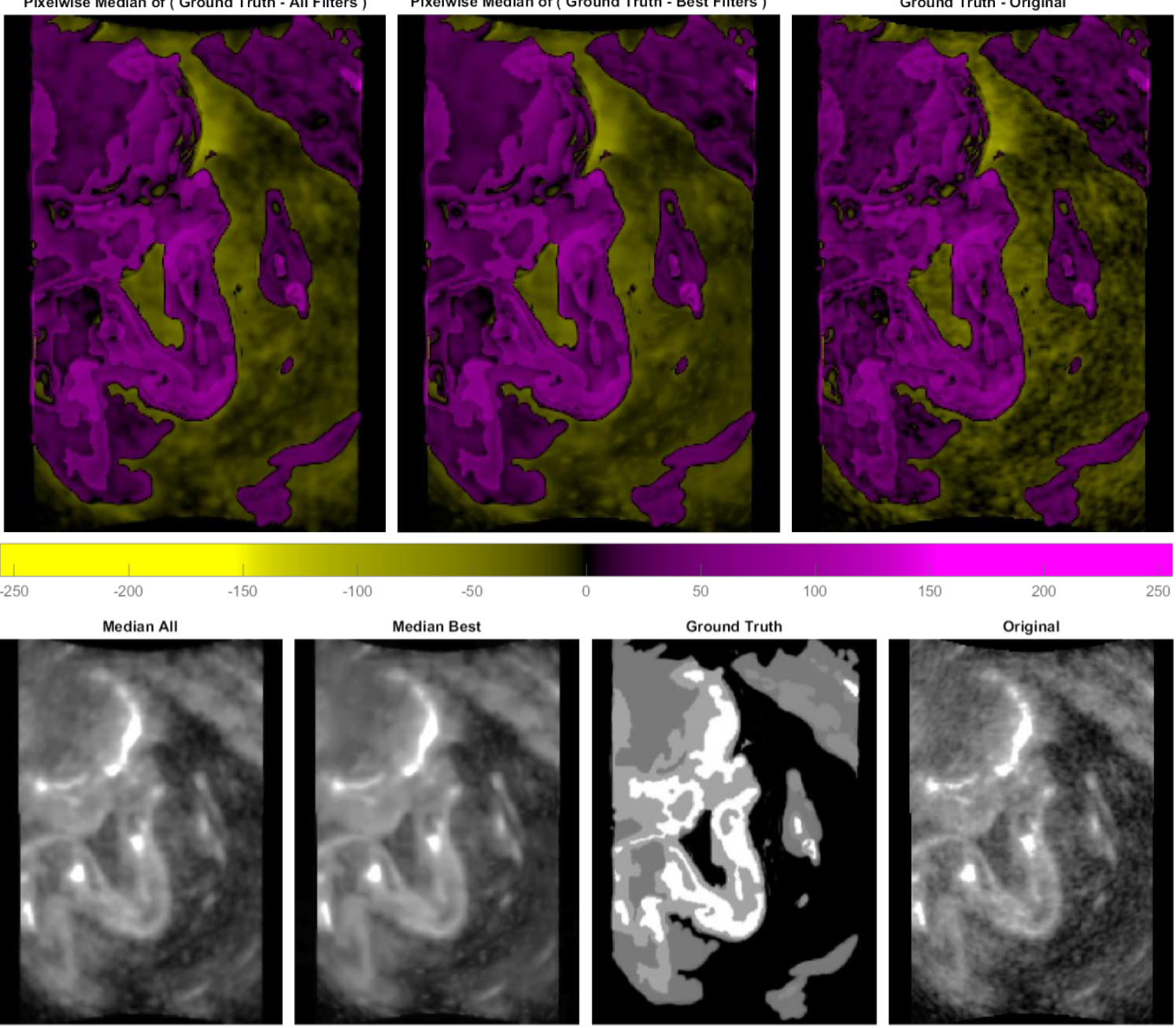

150

Ground Truth

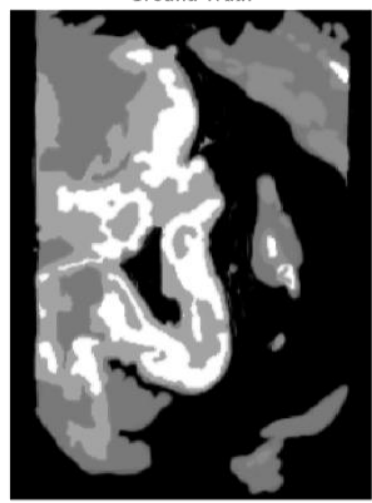

Original

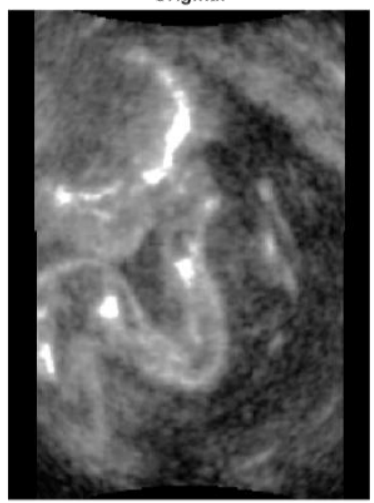

Figura 9.13 - Na linha superior estão as imagens obtidas pela subtração da imagem Ground Truth Testcase13_11 com a mediana, pixel a pixel, do melhor resultado todos os filtros, enquanto na imagem central está a mesma subtração, mas neste caso somente com o melhor resultado dos filtros PPB_Nakagami, SADCTD, SARBM3D, BLF_L0, DSDN, L0 e FoE_Naka e na imagem à direita está a subtração da imagem ground truth com a imagem original. $\mathrm{Na}$ linha inferior encontram-se as imagens medianas respectivas, assim como as imagens de Grouth Truth e Original. 
Pixelwise Median of ( Ground Truth - All Filters )

Pixelwise Median of ( Ground Truth - Best Filters )

Ground Truth - Original
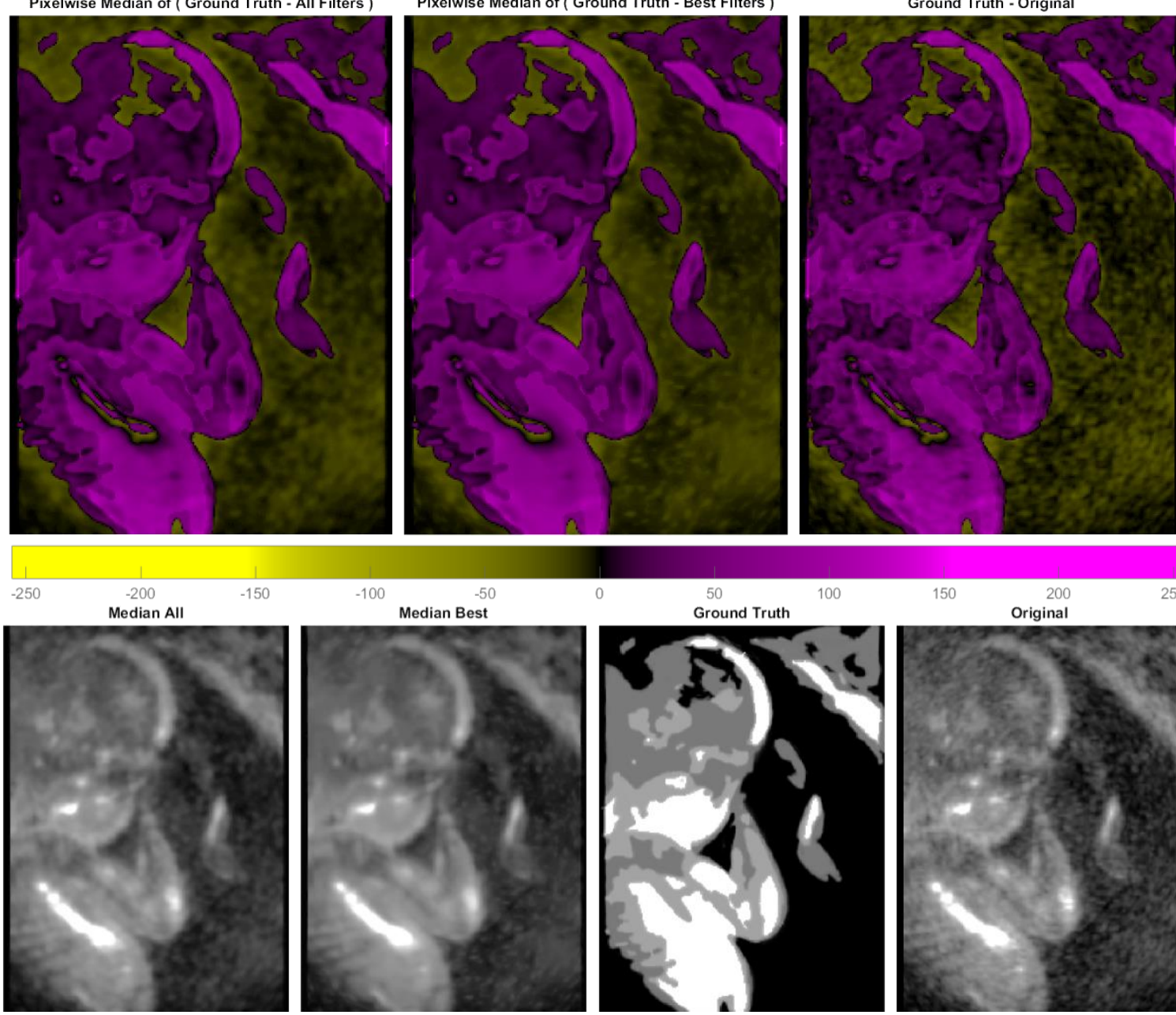

150

Original

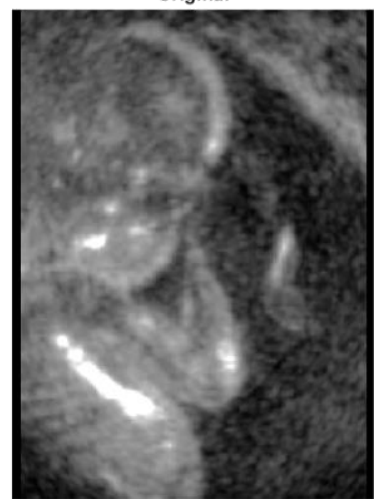

Figura 9.14 - Na linha superior estão as imagens obtidas pela subtração da imagem Ground Truth Testcase13_28 com a mediana, pixel a pixel, do melhor resultado todos os filtros, enquanto na imagem central está a mesma subtração, mas neste caso somente com o melhor resultado dos filtros PPB_Nakagami, SADCTD, SARBM3D, BLF_LO, DSDN, LO e FoE_Naka e na imagem à direita está a subtração da imagem ground truth com a imagem original. Na linha inferior encontram-se as imagens medianas respectivas, assim como as imagens de Grouth Truth e Original. 

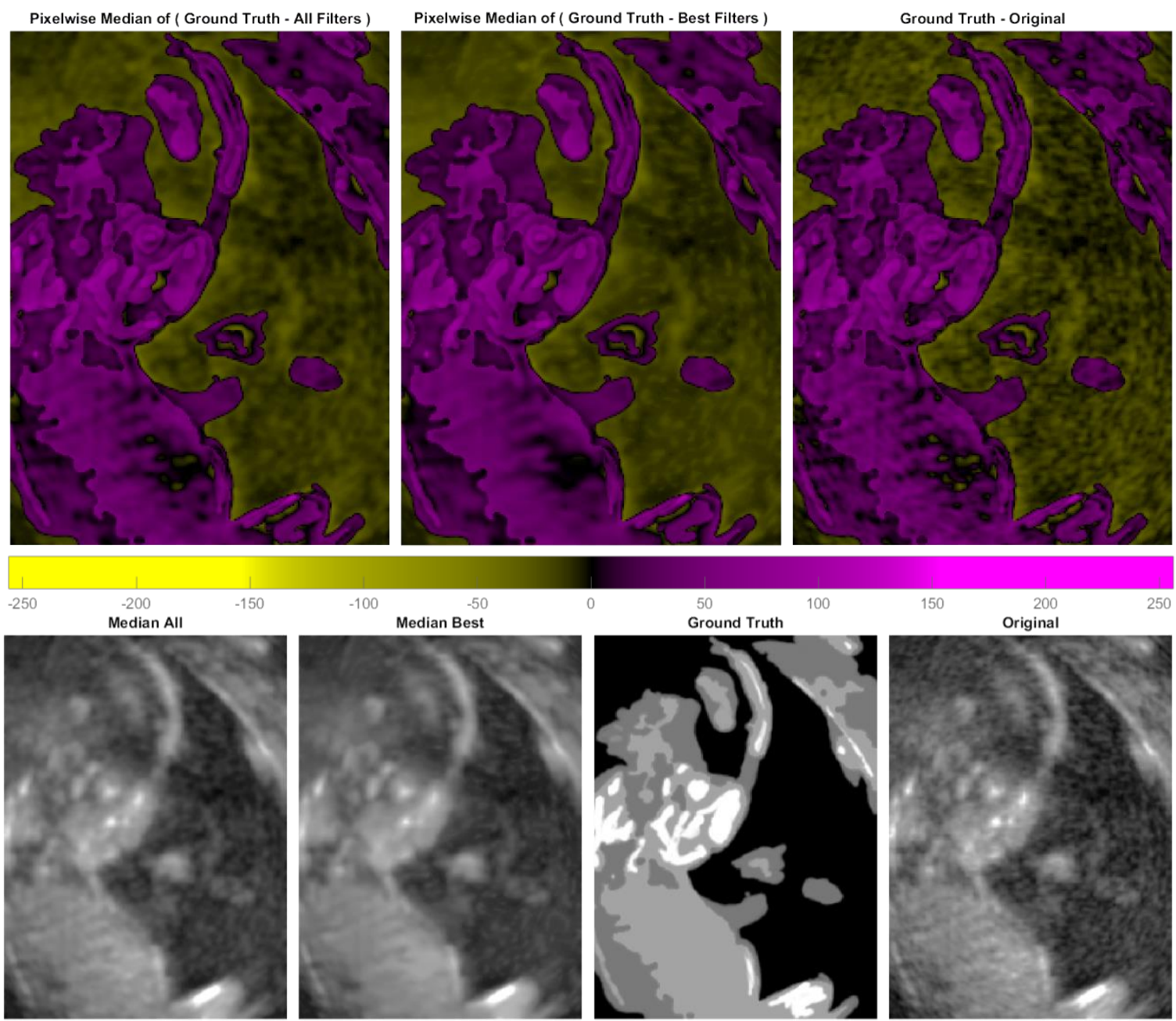

Figura 9.15 - Na linha superior estão as imagens obtidas pela subtração da imagem Ground Truth Testcase13_47 com a mediana, pixel a pixel, do melhor resultado todos os filtros, enquanto na imagem central está a mesma subtração, mas neste caso somente com o melhor resultado dos filtros PPB_Nakagami, SADCTD, SARBM3D, BLF_L0, DSDN, LO e FoE_Naka e na imagem à direita está a subtração da imagem ground truth com a imagem original. $\mathrm{Na}$ linha inferior encontram-se as imagens medianas respectivas, assim como as imagens de Grouth Truth e Original. 
Pixelwise Median of ( Ground Truth - All Filters )

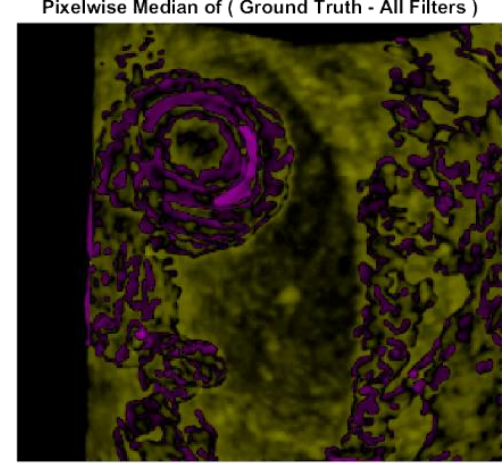

Pixelwise Median of ( Ground Truth - Best Filters )

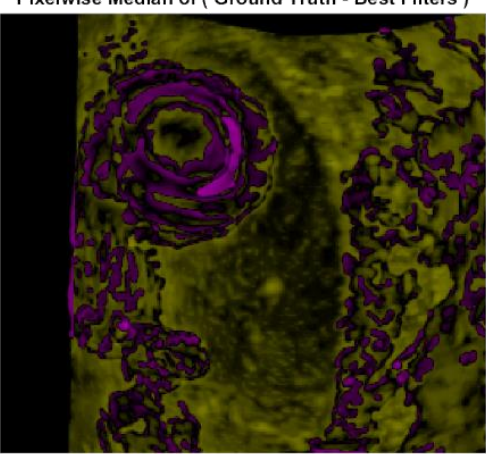

Ground Truth - Original
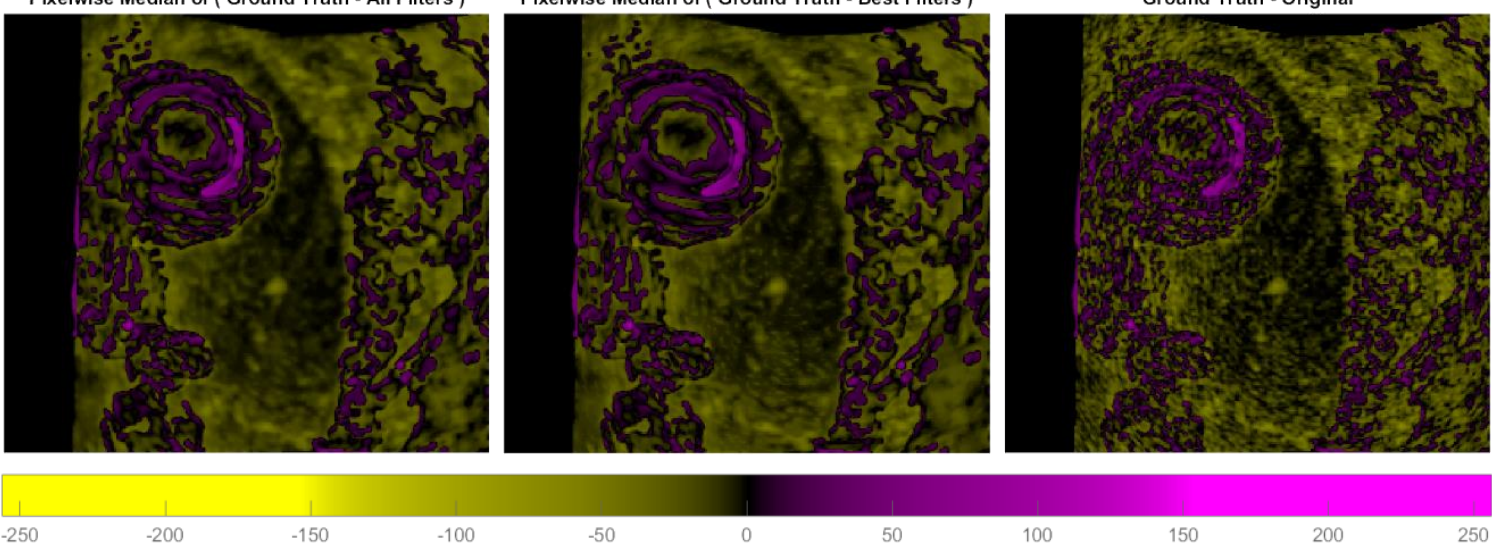

50

100

150

200

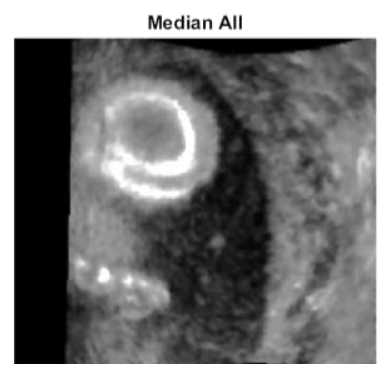

Median Best
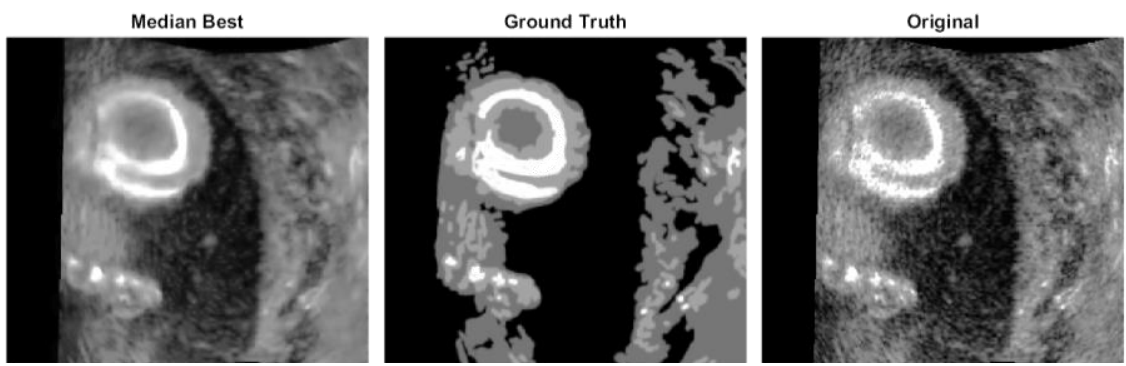

Figura 9.16 - Na linha superior estão as imagens obtidas pela subtração da imagem Ground Truth Testcase14_12 com a mediana, pixel a pixel, do melhor resultado todos os filtros, enquanto na imagem central está a mesma subtração, mas neste caso somente com o melhor resultado dos filtros PPB_Nakagami, SADCTD, SARBM3D, BLF_LO, DSDN, LO e FoE_Naka e na imagem à direita está a subtração da imagem ground truth com a imagem original. Na linha inferior encontram-se as imagens medianas respectivas, assim como as imagens de Grouth Truth e Original. 

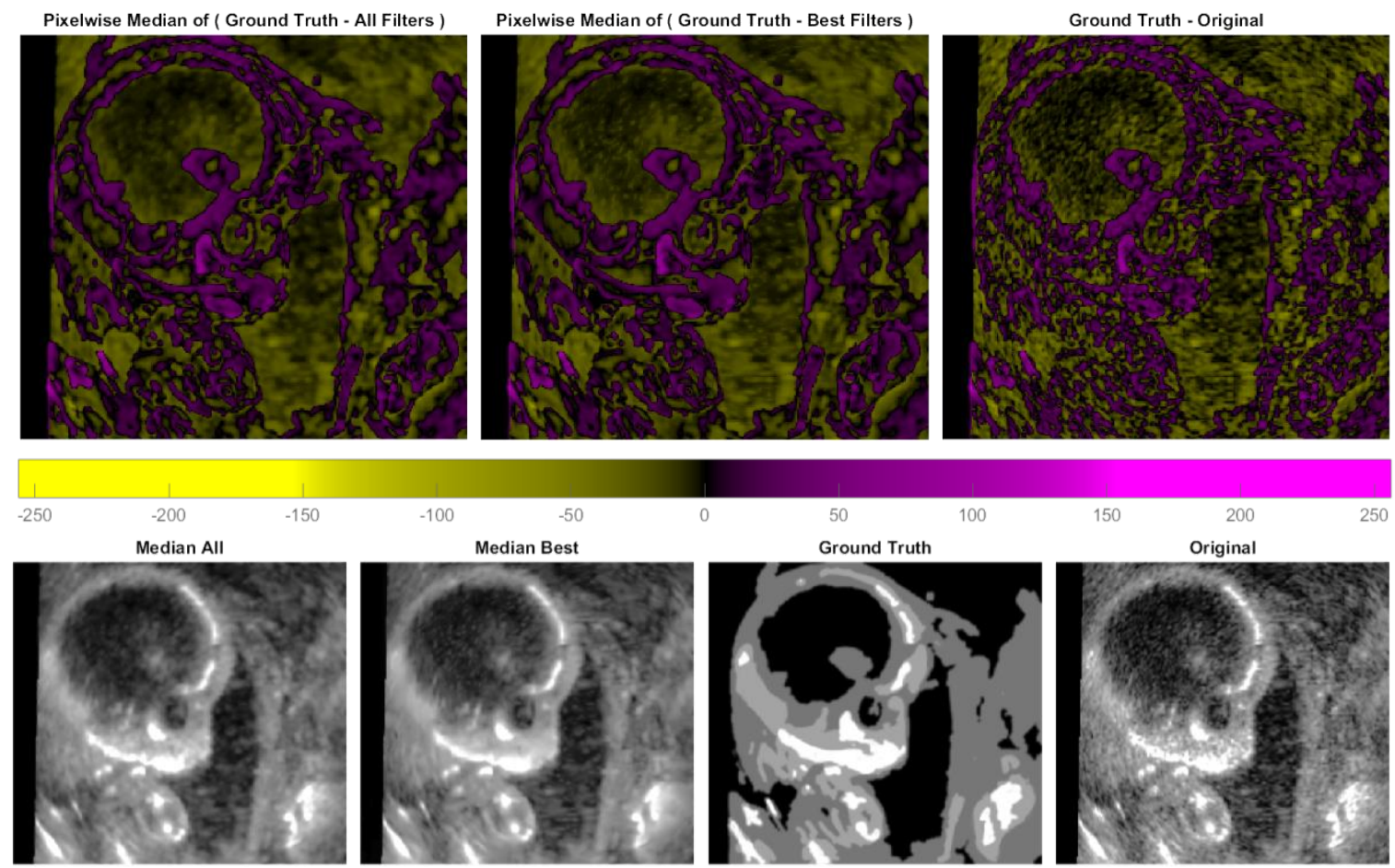

Figura 9.17 - Na linha superior estão as imagens obtidas pela subtração da imagem Ground Truth Testcase14_36 com a mediana, pixel a pixel, do melhor resultado todos os filtros, enquanto na imagem central está a mesma subtração, mas neste caso somente com o melhor resultado dos filtros PPB_Nakagami, SADCTD, SARBM3D, BLF_L0, DSDN, LO e FoE_Naka e na imagem à direita está a subtração da imagem ground truth com a imagem original. Na linha inferior encontram-se as imagens medianas respectivas, assim como as imagens de Grouth Truth e Original. 
Pixelwise Median of ( Ground Truth - All Filters )
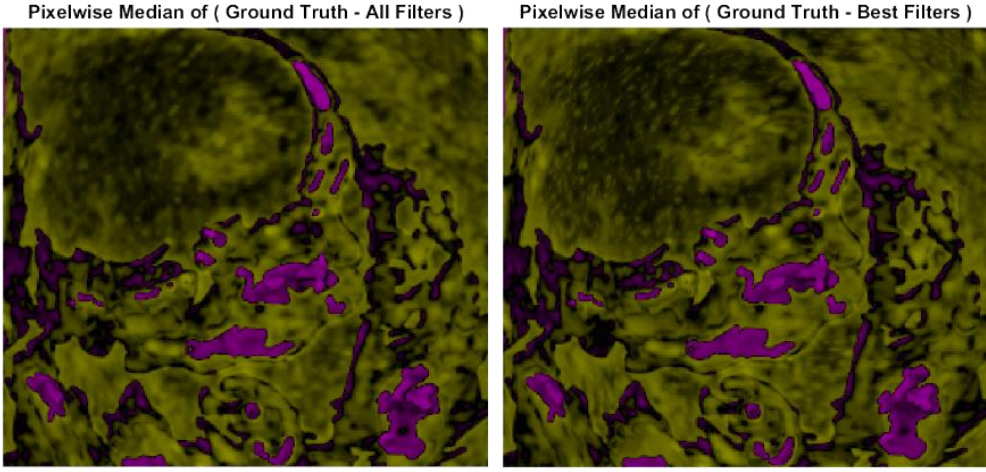

Ground Truth - Original
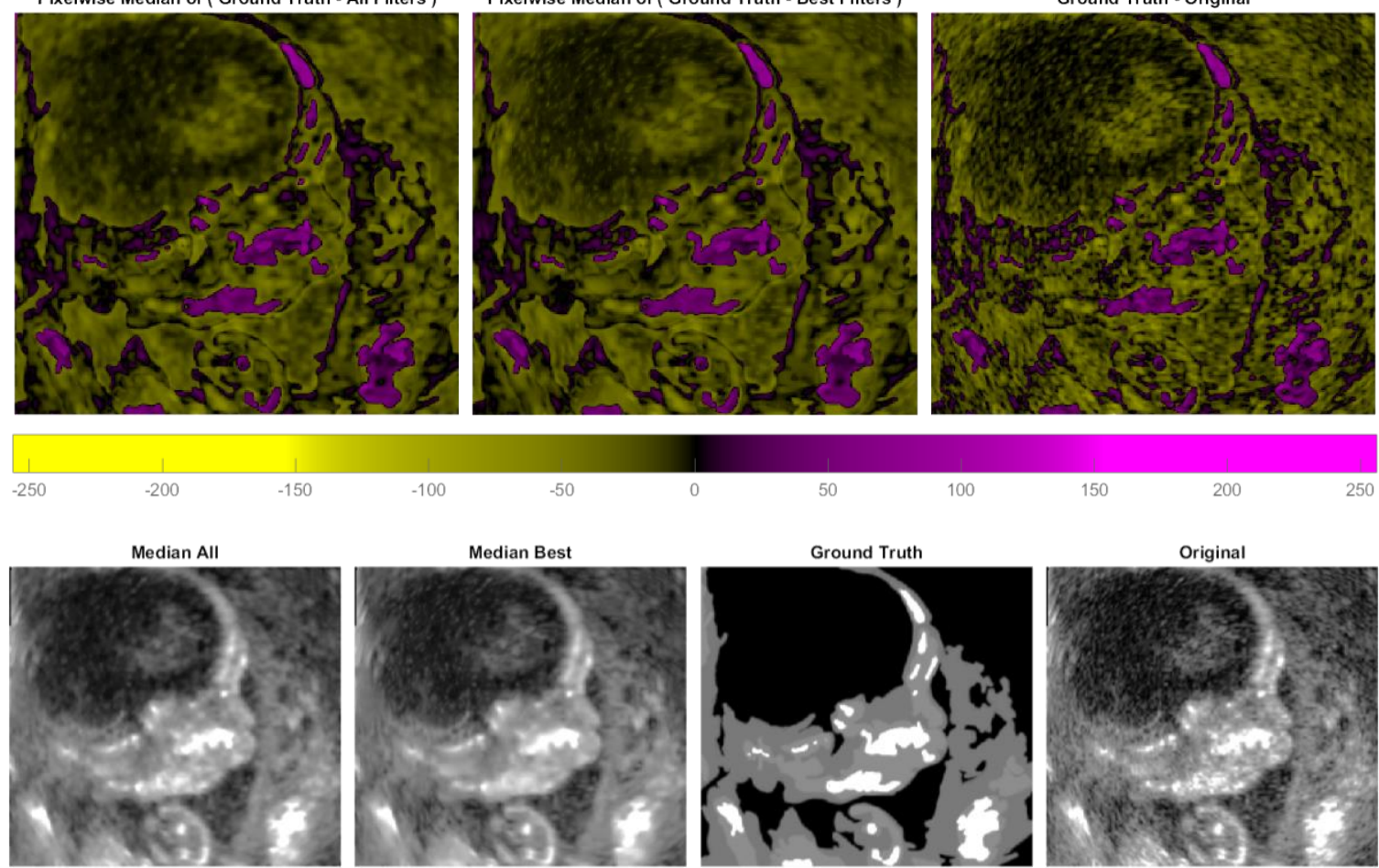

Figura 9.18 - Na linha superior estão as imagens obtidas pela subtração da imagem Ground Truth Testcase14_59 com a mediana, pixel a pixel, do melhor resultado todos os filtros, enquanto na imagem central está a mesma subtração, mas neste caso somente com o melhor resultado dos filtros PPB_Nakagami, SADCTD, SARBM3D, BLF_L0, DSDN, LO e FoE_Naka e na imagem à direita está a subtração da imagem ground truth com a imagem original. Na linha inferior encontram-se as imagens medianas respectivas, assim como as imagens de Grouth Truth e Original. 